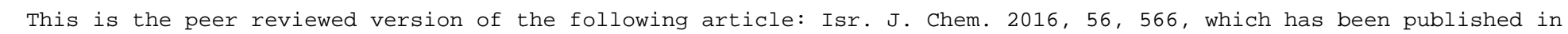

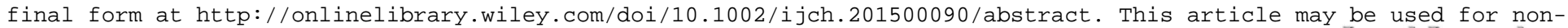
commercial purposes in accordance With Wiley-VCH Terms and Conditions for self-archiving Isrgel Journgl of Chemistry

DOI: $10.1002 /$ ijch.200((full DOI will be filled in by the editorial staff))

\title{
Nucleoside Analogues: Synthesis from Strained Rings
}

\author{
Sophie Racine, ${ }^{[a]}$ Jérémy Vuilleumier and Jérôme Waser*[a] \\ Dedication((optional))
}

\begin{abstract}
Nucleoside analogues are widely employed as bioactive compounds against cancer and viral infections. Consequently, it is important to develop efficient synthetic methods to access them with high efficiency and structural diversity. Herein, we present a full account of our work on the synthesis of nucleoside analogues via annulations of donor acceptor aminocyclopropanes and aminocyclobutanes. Thymine- and uracil- derived diester cyclopropanes were accessed from the corresponding nucleobases via vinylation and rhodium-catalyzed cyclopropanation, and were then used in $(3+2)$ annulations with aldehydes, ketones and enol ethers.
\end{abstract}

The obtained analogues could be transformed into important hydroxymethyl derivatives. Thymine and fluoro-uracil derived diester cyclobutanes obtained from the nucleobases via vinylation and $(2+2)$ cycloaddition could also be used in a $(4+2)$ annulation with aldehydes. Finally, purine-derived diester cyclopropanes could be accessed using the condensation of nucleobases with chloromethyl ethylidene malonates, but annulation reactions with this class of substrates was not successful.

Keywords: Nucleosides • Cyclopropanes $•$ Annulations $•$ Synthetic Method $•$ Catalysis

\section{Introduction}

Nucleoside analogues are widely employed as bioactive compounds against a broad diversity of illnesses such as HIV and different types of cancer. Nevertheless, the emerging resistance towards nucleoside-based antiviral and anticancer agents is alarming. ${ }^{[1]}$ Therefore, the development of new bioactive nucleoside analogues is urgently needed. Nucleosides exhibiting biological activity have structurally diverse cores, including the most common tetrahydrofuranyl amines such as the FDA approved drugs azidothymidine (1) and didanosine (2), and more rare six carbon based scaffolds such as the antiviral compounds anydrohexitolg (3) and cyclohexenyl-g (4). Carbonucleoside analogues such as the antiviral compounds aristeromycin (5), abacavir (6) and cyclohexenyl-g (4) are also of great interest. In comparison with the corresponding tetrahydrofuranyl amine derivatives, they display increased metabolic stability.

Despite the fact that nucleoside analogues are of high interest and were extensively studied, there are only few methods for accessing them with high diversity and efficiency. ${ }^{[2]}$ Nucleoside analogues are generally synthesized using the Vorbrüggen reaction. ${ }^{[2 \mathrm{a}]}$ This transformation consists of the substitution of a leaving group on the ribose by a nucleobase, providing compounds derived from natural ribose. This method allows variation of the nucleobase. However, if unnatural modifications of the ribose core are desired, multistep procedures are usually required. ${ }^{[3]}$ A frequently used approach to access carbonucleoside analogues is to use the Vince lactam, ${ }^{[3 a, b]}$ which after opening delivers a cyclopentenylamine precursor for carbonucleoside derivatives.

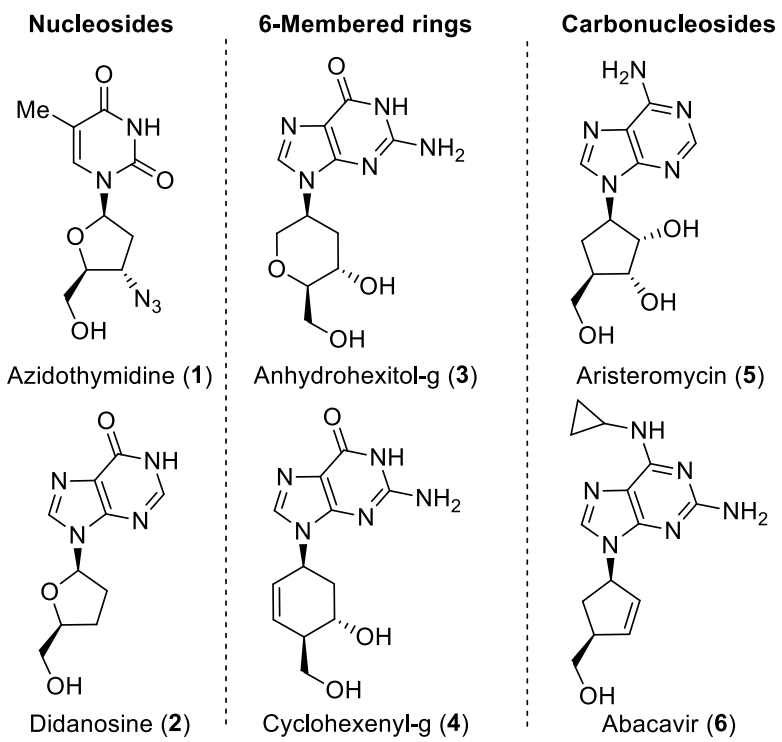

Figure 1. Bioactive nucleoside analogues.

[a] Sophie Racine, Jérémy Vuilleumier, Prof. Dr. Jérôme Waser SB ISIC LCSO, EPFL

1015 Lausanne, $\mathrm{CH}$ phone: +41(0)216939388

fax: +41(0)216939700

e-mail: jerome.waser@epfl.ch

Supporting information for this article is available on the WWW under

http://dx.doi.org/10.1002/ijch.201xxxxxx.((Please

delete if not appropriate)) 
Further elegant work to access nucleoside analogues with a cyclopentyl core have been developed during the last years, proceeding via Pauson-Khand reaction, ${ }^{[\mathrm{c}]}$ ring-closing methathesis ${ }^{[3 \mathrm{~d}]}$ or desymetrization of meso compounds such as cyclopentadiene or diols. ${ }^{[3 \mathrm{e}-\mathrm{g}]}$ Although these novel synthesis strategies increased the diversity of scaffolds accessible, they are often substrate specific and their scope is typically narrow.

Consequently, we decided to to develop a straightforward synthetic method for the synthesis of nucleoside analogues with broad structural diversity. Our goal was to take advantage of the strain release upon ring-opening of small rings such as cyclopropanes and cyclobutanes. Indeed 28 $\mathrm{kcal} / \mathrm{mol}$ of energy is released during the ring opening of cyclopropane, which can be used to initiate chemical transformations. Donor-acceptor (DA) cyclopropanes and DA cyclobutanes in particular are versatile substrates as selective C-C bond cleavage is possible under mild conditions. ${ }^{[4]}$ Thanks to the induced bond polarization, they can act as formal 1,3-dipoles and are suitable substrates for annulation with dipolarophiles providing an effective method for the synthesis of highly substituted five- or six-membered rings. Nitrogen-based DA cyclopropanes have been extensively studied in our group. ${ }^{[5]}$ During these studies, we discovered that the protecting group on the nitrogen atom is key in the success of the annulation reactions. In particular, phthalimide-substituted DA cyclopropanes and cyclobutanes were excellent precursors for the synthesis of tetrahydrofuranyl and tetrahydropyranyl amines and cyclopentyl and cyclohexyl amines via formal (4+2) and $(3+2)$ annulations (Scheme 1, A). In order to apply this methodology to the synthesis of nucleoside analogues, deprotection of the phthalimide group is required to introduce the nucleobase. Unfortunately, the removal of the phthalimide on tetrahydrofuranyl amines was not possible. Furthermore, even if the deprotection worked for cyclopentyl amines, the poor convergence of this strategy led us to design a new method for the synthesis of nucleoside analogues.

The new strategy consisted of having the nucleobase directly linked to the small ring, serving as the donor group (Scheme $1, \mathbf{B})$. With thymine and uracil-substituted DA cyclopropanes and cyclobutanes, five or six membered ring nucleoside analogues become accessible through $(3+2)$ or $(4+2)$ annulations with dipolarophiles such as ketones, aldehydes or enol ethers in a single step ${ }^{[6]}$ Herein, we would like to give a full account of this work, including details on the synthesis of the cyclopropanes, reaction optimization and scope of the annulation reactions. First attempts towards the synthesis of purine-based nucleoside analogues will be also described. At this stage, the nucleoside analogues are only obtained as racemic mixtures, but this could also be an advantage if both enantiomers are needed for biological testing.

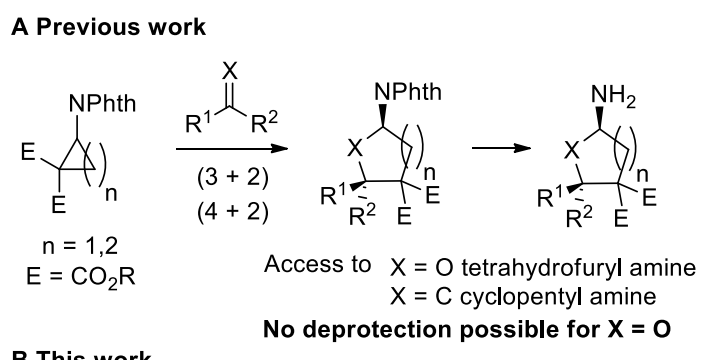

B This work

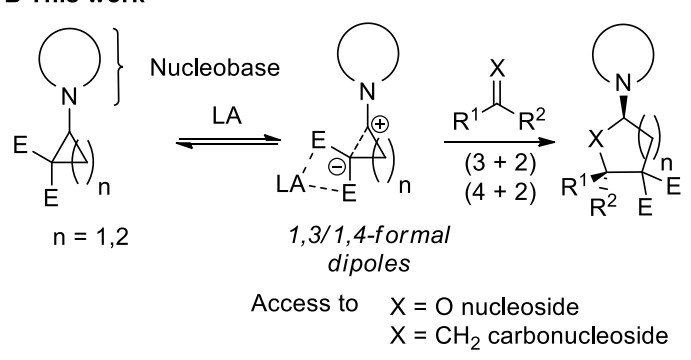

Scheme 1. Strategy to access nucleoside analogues from nitrogen-substituted strained rings.

\section{Synthesis of Nucleoside Analogues via Annulation reactions}

We planned to synthesize the required pyrimidine cyclopropanes via cyclopropanation of the corresponding enamides. Therefore, a robust synthesis of vinyl pyrimidines was needed. When we started our work there was only a few reports of vinylation methods applied to pyrimidine nucleobases: $\mathrm{N}(1)$ alkylation followed by elimination, ${ }^{[7]}$ Chan-Lam coupling using trimethoxyvinyl silane as vinyl source along with tetrabutyl ammonium fluoride (TBAF) and stoichiometric amount of $\mathrm{Cu}(\mathrm{II}),{ }^{[8]}$ and nucleophilic attack of TMS-protected thymine on vinyl acetate in presence of trimethylsilyl trifluoromethane sulfonate (TMSOTf) ${ }^{[9]}$ In our hands the preparation of $\mathrm{N}(1)$ vinyl thymine $\mathbf{1 0 a}$ following these reported procedures failed (Scheme 2). With dibromoethane, we were not able to isolate the desired product $\mathbf{8}$ (Scheme $2, \mathbf{A}$ ). This is in agreement with a later report of Nawrot and co-workers, who showed that alkylation of thymine under these conditions only led to bicyclic side products. ${ }^{[10]}$ Even after intensive optimization, the desired products could be obtained only in $10 \%$ yield. Some conversion was observed in the Chan-Lam coupling leading to a mixture of $\mathrm{N}(3)$ and $\mathrm{N}(1)$ vinylated products 9 and 10a, which were isolated in $24 \%$ and $13 \%$ yield respectively with $\mathrm{N}(3)$ vinylated thymine (9) as major product (Scheme 2, B). In addition to the low yield of the N(1) vinyl thymine (10a), the separation of the regioisomers was laborious, prohibiting the use of this reaction for the preparation of larger amounts of $\mathrm{N}(1)$ vinyl thymine (10a). In conclusion, the reported procedures were not suitable to prepare enough $\mathrm{N}(1)$ vinyl thymine (10a) for the further development of our methodology. 
A

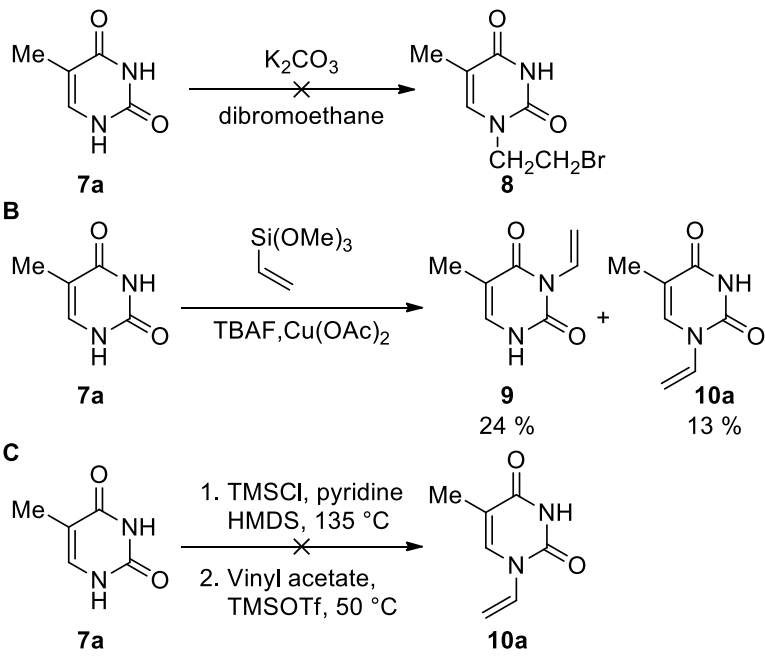

Scheme 2. Attempts for the preparation of $\mathrm{N}(1)$ vinyl thymine using reported procedures.

In a precedent report of our group, we described the preparation of vinyl phthalimide, maleimide and succinimide via palladium cross coupling. ${ }^{[5 e]}$ For this transformation, the palladium(II) sources used were sodium tetrachloropalladate or palladium dichloride with lithium chloride. However, these transformations were performed in vinylacetate (11) as solvent in which thymine (7a) is not soluble. Solubility experiments with different co-solvents were conducted with dichloromethane (DCM), dichloroethane (DCE), chloroform, methanol $(\mathrm{MeOH})$, ethanol $(\mathrm{EtOH})$, acetonitrile $(\mathrm{ACN})$, tetrahydrofuran (THF), dioxane, dimethylformamide (DMF) and water. The solubility of thymine (7a) was highest in hot water and hot mixtures of alcohol $(\mathrm{MeOH}$ or $\mathrm{EtOH})$ and water $\left(60{ }^{\circ} \mathrm{C}\right.$ ), followed by dioxane and DMF (both at $60{ }^{\circ} \mathrm{C}$ ) in which no complete dissolution was observed. Attempts at palladium coupling in dioxane were not successful, but better results were obtained in DMF. $\mathrm{Na}_{2} \mathrm{PdCl}_{4}$ or $\mathrm{PdCl}_{2}$ with or without $\mathrm{LiCl}$ were not suitable catalysts for the vinylation of thymine (7a) (Table 1, entries 1 to 3 ): only traces of $\mathrm{N}(1)$ vinyl thymine (10a) were detected (a mixture of $\mathrm{N}(3)$ and $\mathrm{N}(1)$ vinyl thymine 9 and 10a is observed with $\mathrm{PdCl}_{2}$ (entry 1)). With $\mathrm{Pd}(\mathrm{OAc})_{2}$ only $3 \%$ of conversion was observed, but selectively to $\mathrm{N}(1)$ vinylthymine (10a) (entry 4). No dependence on the equivalents of vinyl acetate (11) used was observed (entries 5 and 6). Further investigation with increased amount of $\mathrm{Pd}(\mathrm{OAc})_{2}$ showed that higher loading in palladium led to better conversion (entries 7 and 8). Inspired by the work of Toti et al. ${ }^{[9]}$ and Nawrot et al., ${ }^{[10]}$ who reported the alkylation or vinylation of thymine (7a) in presence of TMSOTf, we performed the reaction with a stoichiometric amount of TMSOTf. The conversion increased from $3 \%$ to $17 \%$ with $4 \%$ of $\mathrm{Pd}(\mathrm{OAc})_{2}$ (entry 9$)$. When the amount of TMSOTf was increased to 2.4 equivalents and the reaction time was extended to $24 \mathrm{~h}$, the reaction conversion reached $32 \%$ and $51 \%$ respectively (entries 10 and 11 ).
Table 1. Screening of reaction conditions for $\mathrm{N}(1)$ vinylation of thymine $(7 a)$.

\begin{tabular}{|c|c|c|c|c|c|c|}
\hline \multirow{2}{*}{$\frac{\text { Entry }}{1}$} & Catalyst & \multicolumn{2}{|c|}{$\mathrm{H}_{\mathrm{O}} \frac{\mathrm{Pd}(\mathrm{II}){ }^{\mathrm{AcO}} \mathbf{\mathrm { DMF }}, 70^{\circ} \mathrm{C}}{\text { Loading }^{[\mathrm{a}]} \text { Additive }^{[\mathrm{b}]}}$} & \multicolumn{2}{|c|}{ equiv 11 Time } & Ratio ${ }^{[c]}$ \\
\hline & $\mathrm{PdCl}_{2}$ & 4 & - & 1.2 & $10 \mathrm{~h}$ & $<2 \%\left[{ }^{[d]}\right.$ \\
\hline 2 & $\mathrm{Na}_{2} \mathrm{Pd}_{2} \mathrm{Cl}_{4}$ & 4 & - & 1.2 & $10 \mathrm{~h}$ & $<2 \%$ \\
\hline 3 & $\mathrm{PdCl}_{2}$ & 4 & $\mathrm{LiCl}$ & 1.2 & $24 \mathrm{~h}$ & $<2 \%$ \\
\hline 4 & $\mathrm{Pd}(\mathrm{OAc})_{2}$ & 4 & - & 1.2 & $24 \mathrm{~h}$ & $3 \%$ \\
\hline 5 & $\mathrm{Pd}(\mathrm{OAc})_{2}$ & 4 & - & 1.6 & $10 \mathrm{~h}$ & $3 \%$ \\
\hline 6 & $\mathrm{Pd}(\mathrm{OAc})_{2}$ & 4 & - & 2 & $10 \mathrm{~h}$ & $3 \%$ \\
\hline 7 & $\mathrm{Pd}(\mathrm{OAc})_{2}$ & 7 & - & 1.2 & $10 \mathrm{~h}$ & $6 \%$ \\
\hline 8 & $\mathrm{Pd}(\mathrm{OAc})_{2}$ & 20 & - & 1.2 & $10 \mathrm{~h}$ & $11 \%$ \\
\hline 9 & $\mathrm{Pd}(\mathrm{OAc})_{2}$ & 4 & TMSOTf & 1.2 & $10 \mathrm{~h}$ & $17 \%$ \\
\hline 10 & $\mathrm{Pd}(\mathrm{OAc})_{2}$ & 4 & TMSOTf $^{[\mathrm{e}]}$ & 1.2 & $10 \mathrm{~h}$ & $32 \%$ \\
\hline 11 & $\mathrm{Pd}(\mathrm{OAc})_{2}$ & 4 & TMSOTf[e] & 1.2 & $24 \mathrm{~h}$ & $51 \%$ \\
\hline 12 & $\mathrm{Pd}(\mathrm{OAc})_{2}$ & 4 & $\begin{array}{c}\mathrm{TMSOTf}^{[\mathrm{e}]} \\
\mathrm{NEt}_{3}{ }^{[\mathrm{e}]}\end{array}$ & 1.2 & $24 \mathrm{~h}$ & $83 \%$ \\
\hline
\end{tabular}

Reaction conditions: Thymine $(7 \mathrm{a})(0.1 \mathrm{~g}, 0.8 \mathrm{mmol})$, palladium catalyst and additives in DMF $(2 \mathrm{~mL})$ were stirred in a flamedried flask under nitrogen and heated to $70^{\circ} \mathrm{C}$. [a] In mol\%. [b] 1.2 equiv. [c] NMR ratio $(\mathbf{1 0 a} /(\mathbf{7 a}+\mathbf{1 0 a}))$ of the integration of the $\mathrm{C}=\mathrm{C}-\mathrm{H}$ thymine signals. [d] Mixture of $\mathrm{N}(1)$ and $\mathrm{N}(3)$ vinyl thymine. [e] 2.4 equiv additive was used.

Finally after adding an additional base (triethylamine) the conversion went to $83 \%$ and the isolated yield reached $79 \%$ of the sole N(1) vinyl-thymine (10a) (entry 12). Fortunately, the vinylation worked well also on uracil (7b) and 5-fluorouracil (7c) with complete regioselectivity and respectively $69 \%$ and $57 \%$ isolated yield (products $\mathbf{1 0 b}$ and $\mathbf{1 0 c}$ ).

To access the desired cyclopropanes, protection of the imide nitrogen was performed, followed by cyclopropanation using the corresponding diazomalonate and the rhodium Espino catalyst developed by Du Bois and co-workers (Scheme 3). ${ }^{[11]}$ For thymine (7a) and uracil (7b), a tert-butoxycarbonyl protecting group was used. When protection of fluoro-uracil (7c) was attempted, a mixture of carbonates and carbamate was obtained in poor yield. In this case a benzoyl group could be installed selectively on the nitrogen. The three pyrimidine cyclopropanes 13a, 13b and 13c were obtained in good overall yields. 


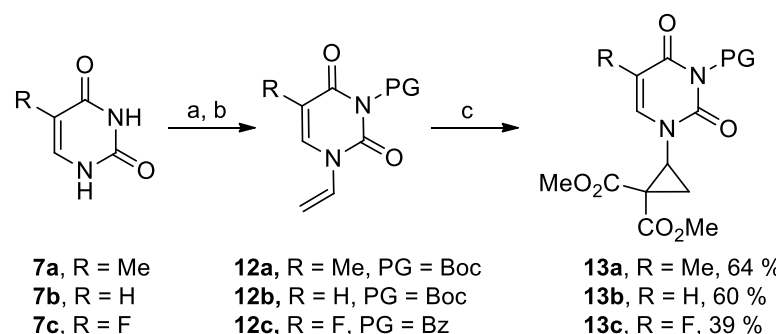

Scheme 3. Synthesis of cyclopropanes 13. Reaction conditions: a) $4 \mathrm{~mol} \% \mathrm{Pd}(\mathrm{OAc})_{2}$, vinylacetate, TMSOTf, $\mathrm{NEt}_{3}, 70{ }^{\circ} \mathrm{C}$, DMF. b) $\mathrm{Boc}_{2} \mathrm{O}, \mathrm{DMAP}, \mathrm{CH}_{2} \mathrm{Cl}_{2}$ or $\mathrm{BzCl} \mathrm{DMAP}, \mathrm{NEt}_{3}$. c) $0.02 \mathrm{~mol} \%$ $\mathrm{Rh}_{2}(\mathrm{esp})_{2}$, diazodimethylmalonate, $\mathrm{CH}_{2} \mathrm{Cl}_{2}$.

Surprisingly, when the cyclopropanation of vinyl-uracil $\mathbf{1 2 b}$ was performed with 1.2 equivalents instead of an equimolar amount of the diazomalonate, a new pyrimidine cyclopropane was isolated containing two malonate moieties with an intact thymine double bond (Scheme 4). After extensive spectroscopic investigations, we identified this compound as 14. The formation of cyclopropane $\mathbf{1 4}$ can be tentatively explained by the unusual formation of epoxide $\mathbf{I}$ instead of cyclopropanation of the thymine double bond through the reaction with one additional equivalent of diazomalonate mediated by the rhodium Espino catalyst. Then, an intramolecular rearrangement via opening of the epoxide, followed by the migration of the Boc group to the oxygen atom would afford pyridone cyclopropane $\mathbf{1 4}$.

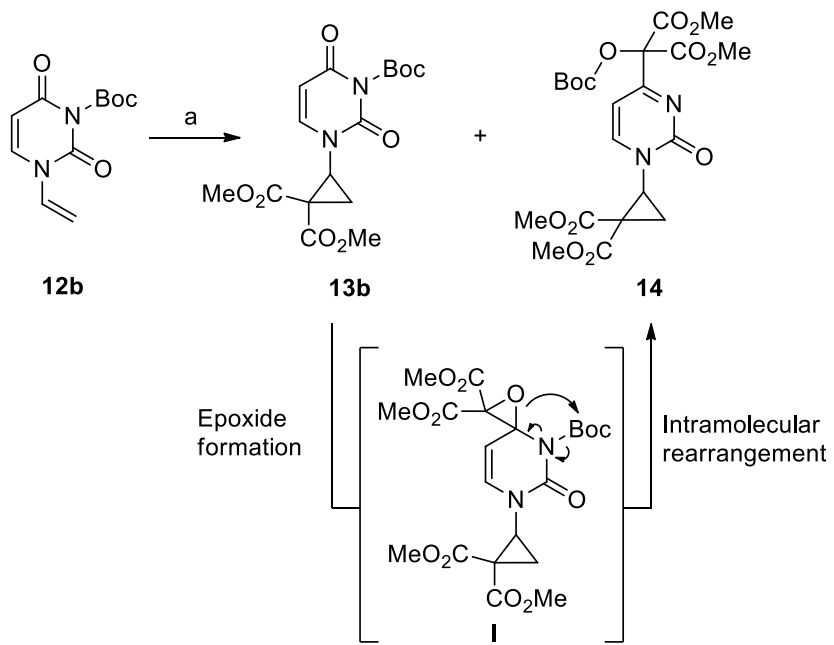

Scheme 4. Unexpected formation of pyridone cyclopropane 14 and tentative mechanism. Reaction conditions: a) $0.5 \mathrm{~mol} \%$ $\mathrm{Rh}_{2}(\mathrm{esp})_{2}, 1.2$ equiv diazodimethylmalonate, $\mathrm{CH}_{2} \mathrm{Cl}_{2}$. Yields: $\mathbf{1 3} \mathbf{b}$ $28 \%$ and $1464 \%$. ${ }^{[12]}$

Starting again from vinyl thymine 10a, a [2+2] cycloaddition gave access to the thymine DA cyclobutane 16a. As the reported procedure of our group to synthesize thymine cyclobutanes through [2+2] cycloaddition using $\mathrm{FeCl}_{3}$ on aluminium oxide as catalyst ${ }^{[5 i]}$ gave low yield (Table 2, entry 1), other Lewis acids were screened. Low conversion was obtained with $\mathrm{Zn}(\mathrm{OTf})_{2}, \mathrm{AuCl}_{3}, \mathrm{Sn}(\mathrm{OTf})_{2}$ and $\mathrm{Sc}(\mathrm{OTf})_{3}$ (entries 2 to 5). Complete decomposition of the starting material was observed when $\mathrm{Hf}(\mathrm{OTf})_{4}$ and $\mathrm{TiCl}_{4}$ were used (entries 6 and 7). With $\mathrm{In}(\mathrm{OTf})_{3}, \mathrm{Cu}(\mathrm{OTf})_{2}$ and $\mathrm{Yb}(\mathrm{OTf})_{3}$ as Lewis acid catalysts, the reaction exhibited low conversion (entries 8 to 10). Nevertheless, using $\mathrm{FeCl}_{3}$ on aluminium oxide the conversion was increased from $13 \%$ after 14 hours to $35 \%$ after 24 hours (entries 1 and 11). The reaction was shut down when other solvents were used such as $\mathrm{MeOH}$, DMF, dioxane, $\mathrm{CCl}_{4}$ or a mixture of $\mathrm{DCM} / \mathrm{MeOH}$. $\mathrm{ACN}$ allows the reaction to proceed, but with lower conversion. Using DCE as solvent gave roughly similar conversion to the DA cyclobutane 16a.

Table 2. Optimization of the $[2+2]$ reaction.

\begin{tabular}{|c|c|c|c|c|c|}
\hline & $10 a$ & & & $16 a$ & \\
\hline Entry & LA & Ratio $^{\text {[a] }}$ & Entry & LA & Ratio[a] $^{[a]}$ \\
\hline 1 & $\mathrm{FeCl}_{3} \cdot \mathrm{Al}_{2} \mathrm{O}_{3}$ & $13 \%$ & 7 & $\mathrm{TiCl}_{4}$ & dec. \\
\hline 2 & $\mathrm{Zn}(\mathrm{OTf})_{2}$ & $<2 \%$ & 8 & $\mathrm{Cu}(\mathrm{OTf})_{2}$ & $5 \%$ \\
\hline 3 & $\mathrm{AuCl}_{3}$ & $<2 \%$ & 9 & $\mathrm{Yb}(\mathrm{OTf})_{3}$ & $5 \%$ \\
\hline 4 & $\mathrm{Sc}(\mathrm{OTf})_{3}$ & $<2 \%$ & 10 & $\ln (\mathrm{OTf})_{3}$ & $10 \%$ \\
\hline 5 & $\mathrm{Sn}(\mathrm{OTf})_{2}$ & $<2 \%$ & 11 & $\mathrm{FeCl}_{3} \cdot \mathrm{Al}_{2} \mathrm{O}_{3}$ & $35 \%{ }^{[b]}$ \\
\hline 6 & $\mathrm{Hf}(\mathrm{OTf})_{4}$ & dec. & & & \\
\hline
\end{tabular}

Reaction conditions: Vinyl thymine $10 \mathrm{a}(50 \mathrm{mg}, 0.33 \mathrm{mmol}), 2$ equiv methylidene malonate 15 , dry $\mathrm{CH}_{2} \mathrm{Cl}_{2}, 0.3 \mathrm{M}$, stirred under $\mathrm{N}_{2}$. [a] NMR ratio $(\mathbf{1 6 a} /(\mathbf{1 0} \mathbf{a}+\mathbf{1 6} \mathbf{a}))$ of the integration of the $\mathrm{C}=\mathrm{C}$ $\mathrm{H}$ signals. [b] 24 hour reaction.

When the reaction is conducted with freshly prepared $\mathrm{FeCl}_{3} \cdot \mathrm{Al}_{2} \mathrm{O}_{3}$ and methylidene malonate on preparative scale, the isolated yield of $\mathbf{1 6 a}$ reached $43 \%$ (Scheme 5). However, $(4+2)$ cycloaddition was not successful with this substrate (vide infra). Thymine cyclobutane $\mathbf{1 6 a}$ was therefore protected with a tert-butoxy carbonyl group to avoid side reactions. However, the Boc group exhibited high lability and was removed in presence of Lewis acids.

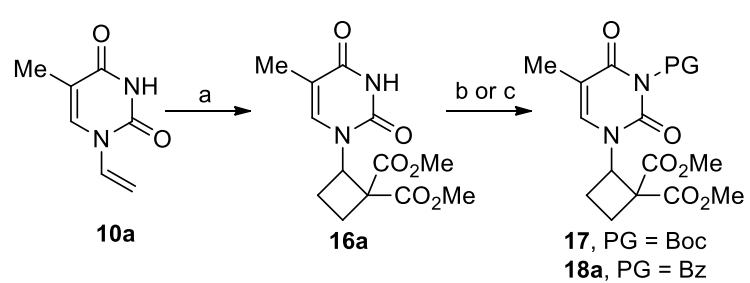

Scheme 5. Synthesis of thymine cyclobutanes. Reaction conditions: a) 0.2 equiv $\mathrm{FeCl}_{3} \cdot \mathrm{Al}_{2} \mathrm{O}_{3}$, methylidene dimethylmalonate (15), DCM, $43 \%$. b) $\mathrm{Boc}_{2} \mathrm{O}$, DMAP, ACN, $81 \%$. c) $\mathrm{BzCl}, \mathrm{DMAP}, \mathrm{NEt}_{3}, \mathrm{DCM}, 86 \%$.

Benzoyl as protecting group was then examined, as it was expected to be more stable than tert-butoxycarbonyl group. Benzoylation of 16a gave protected cyclobutane 18a in $86 \%$ yield. Using the same reaction sequence, fluoro-uracil 
derived cyclobutane $\mathbf{1 8 b}$ could be also obtained in good yield (Scheme 6).

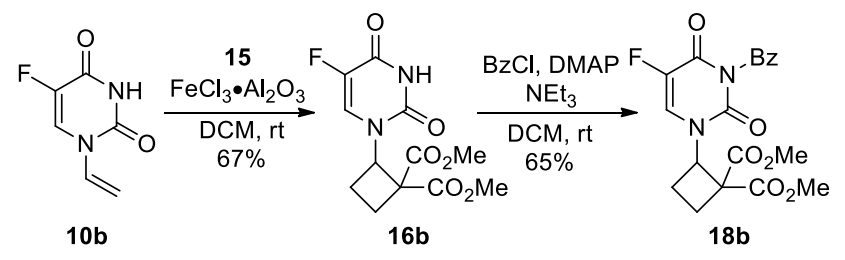

Scheme 6. Preparation of 5-fluoro uracil cyclobutane $\mathbf{1 8 b}$.

Having the pyrimidine-based DA cyclopropanes 13a-c and DA cyclobutanes 18a, $\mathbf{b}$ in hand, we then examined the corresponding $(3+2)$ and $(4+2)$ formal cycloadditions catalyzed by Lewis acids with ketones, aldehydes and enol ethers as partners to access 5- and 6- membered nucleoside analogues. Using the conditions described for the $(3+2)$ reaction with aldehydes and phthalimide-substituted DA cyclopropanes, the desired five-membered ring 22a was isolated in low yield with the thymine-substituted DA cyclopropane 13a (Table 3, entry 1).

Table 3. Optimization of the [3+2] annulation.

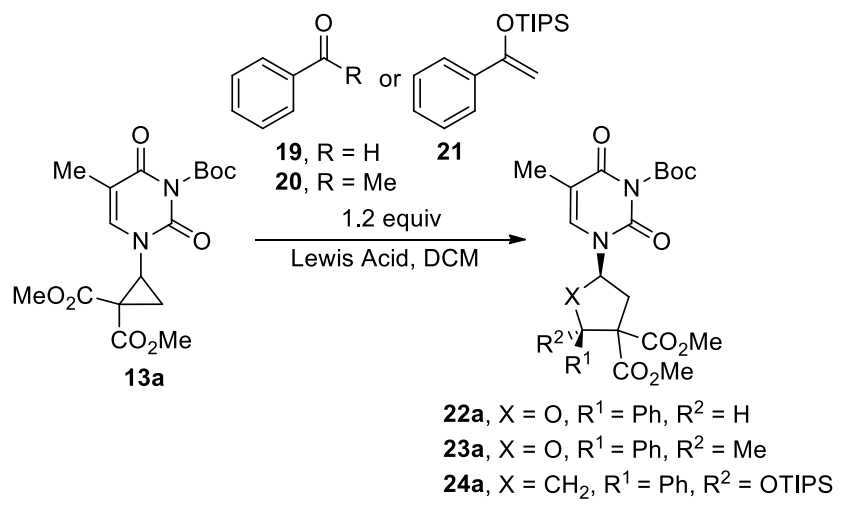

\begin{tabular}{ccccccc}
\hline Entry Partner $^{[a]}$ & LA & Loading $^{[b]}$ & $\mathrm{T}$ & Time & Yield \\
\hline 1 & $\mathbf{1 9}$ & $\mathrm{FeCl}_{3} \cdot \mathrm{Al}_{2} \mathrm{O}_{3}$ & 5 & $\mathrm{rt}$ & 3 days & $27 \%$ \\
2 & $\mathbf{1 9}$ & $\mathrm{In}(\mathrm{OTf})_{3}$ & 20 & $\mathrm{rt}$ & $1 \mathrm{~h}$ & $86 \%$ \\
3 & $\mathbf{1 9}$ & $\mathrm{Hf}(\mathrm{OTf})_{4}$ & 20 & $\mathrm{rt}$ & $1 \mathrm{~h}$ & $49 \%[\mathrm{~d}]$ \\
4 & $\mathbf{2 0}$ & $\mathrm{SnCl}_{4}$ & 5 & $-78{ }^{\circ} \mathrm{C}$ & 2 days & $6 \%$ \\
5 & $\mathbf{2 1}$ & $\mathrm{SnCl}_{4}$ & 10 & $-78{ }^{\circ} \mathrm{C}$ & 2 days & $52 \%$ \\
6 & $\mathbf{2 0}$ & $\mathrm{SnCl}_{4}$ & 5 & $-20^{\circ} \mathrm{C}$ & 30 min & $60 \%$ \\
7 & $\mathbf{2 1}$ & $\mathrm{SnCl}_{4}$ & 10 & $-20{ }^{\circ} \mathrm{C}$ & $30 \mathrm{~min}$ & $60 \%$ \\
\hline
\end{tabular}

[a] Reaction conditions: 1.2 equiv 19,20 or $\mathbf{2 1}$, dry DCM, $0.3 \mathrm{M}$, stirred under $\mathrm{N}_{2}$. [b] In mol\%. [c] Isolated yield. [d] Yield of the nucleoside without the protecting group. Deprotection occurred under these conditions.

When the Lewis acid was changed from $\mathrm{FeCl}_{3} \cdot \mathrm{Al}_{2} \mathrm{O}_{3}$ to $\mathrm{In}(\mathrm{OTf})_{3}$, the isolated yield increased to $86 \%$ (entry 2). Interestingly, with $\mathrm{Hf}(\mathrm{OTf})_{4}$ as catalyst, the yield was moderate, but gave a direct access to the deprotected product 22b (entry 3). However, this yield could not be improved. In our previous work, when ketones and enol ethers were used as dipolarophiles with phthalimide cyclopropanes, the best Lewis acid was $\mathrm{SnCl}_{4}$ at low temperature. ${ }^{[\mathrm{a}-\mathrm{b}]}$ Using these conditions with thymine cyclopropane 13a over two days led to low yield with acetophenone (20) and moderate yield with TIPS protected acetophenone (21) (entries 4 and 5). Increasing the temperature to $-20{ }^{\circ} \mathrm{C}$, resulted in complete conversion after 30 minutes (entries 6 and 7). The two desired nucleoside analogues $\mathbf{2 3 a}$ and $\mathbf{2 4 a}$, synthesized from the ketone $\mathbf{2 0}$ and the enol ether 21, respectively, were isolated in $60 \%$ yields.

As the Boc group on thymine is thermally labile, an efficient two step procedure, consisting in the Lewis acid-catalyzed $(3+2)$ annulation, followed by filtration (to remove the Lewis acid catalyst) and tert-butoxycarbonyl removal at $70{ }^{\circ} \mathrm{C}$ for $18 \mathrm{~h}$ was developed (Scheme 7). When the two step procedure was applied to the $(3+2)$ reaction with benzaldehyde $(\mathbf{1 9})$, the unprotected nucleoside 22b was isolated in $87 \%$ yield (Scheme 7, A). The two step protocol gave better yield of nucleoside analogues in the case of acetophenone (20): $94 \%$ yield of 23b was obtained instead of $60 \%$ of 23a without deprotection (Scheme 7, B). Carbonucleoside 24b was isolated in $84 \%$ yield by using the two step procedure.
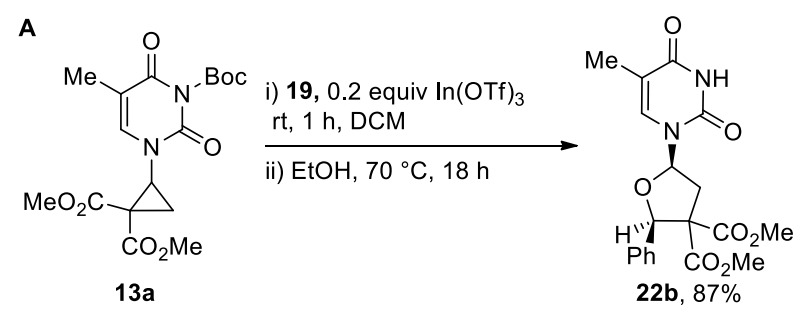<smiles>COC(=O)C1(C(=O)OC)CC1n1cc(C)c(=O)n(C(=O)OCc2ccccc2)c1=O</smiles>

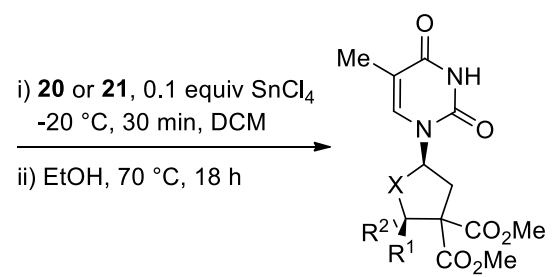

23b, $X=O, R^{1}=P h, R^{2}=M e, 94 \%$

24b, $X=\mathrm{CH}_{2}, \mathrm{R}^{1}=\mathrm{Ph}, \mathrm{R}^{2}=$ OTIPS, $84 \%$

Scheme 7. Optimized conditions for annulation-deprotection.

The $(4+2)$ reaction was then investigated using thymine cyclobutane 16a with different Lewis acids $\left(\mathrm{Zn}(\mathrm{OTf})_{2}, \mathrm{AuCl}_{3}\right.$, $\mathrm{Sn}(\mathrm{OTf})_{2}, \mathrm{Sc}(\mathrm{OTf})_{3} \mathrm{TiCl}_{4}, \mathrm{Hf}(\mathrm{OTf})_{4}, \mathrm{In}(\mathrm{OTf})_{3}, \mathrm{Cu}(\mathrm{OTf})_{2}$, $\mathrm{FeCl}_{3} \cdot \mathrm{Al}_{2} \mathrm{O}_{3}$ and $\left.\mathrm{Yb}(\mathrm{OTf})_{3}\right)$. No conversion was observed for most of the Lewis acids, except with $\mathrm{In}(\mathrm{OTf})_{3}$ and $\mathrm{Hf}(\mathrm{OTf})_{4}$ : these catalysts gave access to tetrahydropyranyl amine in low yield due to degradation. A screening of Lewis acids was also conducted with Boc protected thymine cyclobutane 17, including $\mathrm{Zn}(\mathrm{OTf})_{2}, \mathrm{AuCl}_{3}, \mathrm{Sn}(\mathrm{OTf})_{2}, \mathrm{Sc}(\mathrm{OTf})_{3}, \mathrm{TiCl}_{4}, \mathrm{SnCl}_{4}$, $\mathrm{Hf}(\mathrm{OTf})_{4}, \mathrm{In}(\mathrm{OTf})_{3}, \mathrm{Cu}(\mathrm{OTf})_{2}, \mathrm{FeCl}_{3} \bullet \mathrm{Al}_{2} \mathrm{O}_{3}$ and $\mathrm{Yb}(\mathrm{OTf})_{3}$. Encouraging results were obtained with $\mathrm{Hf}(\mathrm{OTf})_{4}$ and $\mathrm{FeCl}_{3} \cdot \mathrm{Al}_{2} \mathrm{O}_{3}$ : the desired nucleosides analogues were isolated in $59 \%$ and $56 \%$ yield respectively. However, partial loss of the Boc protecting group was again observed. Therefore, we focused our efforts on these two Lewis acids with benzoyl protected thymine cyclobutane 18a. Even in this case, a partial benzoyl group removal was observed, thus deprotection using an ammonium hydroxide solution in ethanol was systematically performed directly after annulation. ${ }^{[13]}$ As benzoyl deprotection was quantitative, the optimization focused on the conditions for the $(3+2)$ 
annulation (Table 4). With hafnium triflate, the two step procedure gave moderate yield after 14 hours (entry 1) due to partial decomposition of the product 25a. When the reaction time was shortened to $30 \mathrm{~min}$, the product $\mathbf{2 5 a}$ was isolated in an increased $76 \%$ yield (entry 2 ). The optimum time was found to be 15 minutes (entry 3), as the conversion is complete and the degradation minimized. If the reaction time was shortened to 10 and 5 minutes (entries 4 and 5), the reaction was not complete and lower yields were obtained. The reaction with iron trichloride supported on alumina went to completion only after 14 hours, providing 25a in moderate yield (entry 6). Therefore, a stoichiometric amount of $\mathrm{FeCl}_{3} \cdot \mathrm{Al}_{2} \mathrm{O}_{3}$ was used in order to shorten the reaction time. Complete conversion was reached after $30 \mathrm{~min}$, but the yield was not improved (entry 7). The same observation was made when the reaction was performed at a $0.3 \mathrm{mmol}$ scale. The reaction was stopped after completion (15 minutes in this case), after filtration and deprotection $60 \%$ yield was obtained (entry 8).

Table 4. Screening of conditions for the [4+2] annulation.

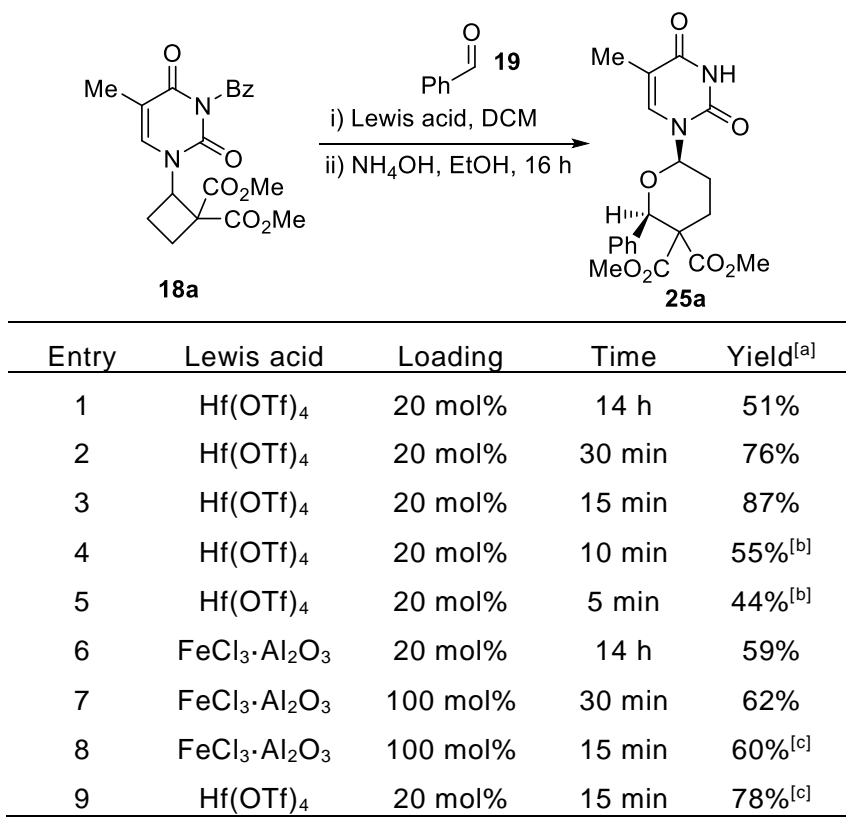

Reaction conditions: $18 \mathrm{a}$ (20 $\mathrm{mg}, 0.050 \mathrm{mmol}), 1.5$ equiv benzaldehyde (19), $0.05 \mathrm{M}$ in dry DCM, stirred under $\mathrm{N}_{2}$. Then filtration, $\mathrm{NH}_{4} \mathrm{OH}, \mathrm{EtOH}, 16$ hours. [a] NMR yields after isolation due to benzamide poisoning (not removable by column), all [4+2] annulations went to full conversion, otherwise stated. [b] [4+2] annulation not complete. [c] $0.3 \mathrm{mmol}$ scale.

Finally, the (4+2) annulation was performed on larger scale with $0.3 \mathrm{mmol}$ of cyclobutane 18a with hafnium triflate, providing the nucleoside analogue $\mathbf{2 5 a}$ in $78 \%$ isolated yield (entry 9). Unfortunately, the (4+2) annulation conducted with TIPS enol ether 21 gave only traces of product even after 24 hours. With acetophenone 20, the desired benzoyl protected nucleoside was obtained in 54\% yield after 24 hours, but the conversion was not complete.

Having the optimized conditions in hand for the $(3+2)$ and the $(4+2)$ annulations, we investigated the scope of the reaction and its limitations. The reaction was examined with aldehydes first (Scheme 8, A). The nucleoside 22c synthesized from 4-fluoroacetophenone, was obtained in $79 \%$ yield and high diastereoselectivity. The diastereoselectivity was lower in the case of cinnamaldehyde, but product 22d was still obtained in $96 \%$ yield. The $(3+2)$ reaction worked also with the sterically hindered aliphatic isobutyraldehyde, providing 22e in $75 \%$ yield and complete diastereoselectivity. We then examined the use of 2-hydroxy acetaldehyde derivatives in the reaction to provide nucleoside analogues 22f-h with the hydroxymethyl group often required for the bioactivity of drugs. Unfortunately, this type of aldehydes was more prompt to polymerization and no conditions to obtain the nucleoside derivatives could be found.

We then turned to ketones as substrates (Scheme 8, B). Aromatic ketones (acetophenone (20) and 4-fluoro acetophenone) afforded the nucleoside analogues $\mathbf{2 3 b}$ and 23c in high yield and diastereoselectivity. In the case of an alkenyl ketone, the product $\mathbf{2 3 d}$ was isolated in good yield and poor diastereoselectivity. Aliphatic ketones also worked well in the annulation reaction: the phenethyl substituted nucleoside derivative $23 \mathbf{e}$ and the bicyclic nucleoside $\mathbf{2 3 f}$ were obtained in $85 \%$ and $97 \%$ yield respectively. Using TBS protected 2-hydroxyacetacetone, nucleoside $\mathbf{2 3 g}$ bearing a hydroxymethyl group could be accessed in $38 \%$ yield.

Carbonucleoside derivatives were then synthesized with enol ethers as dipolarophiles (Scheme 8, C). 24b-d were obtained in high yield and diastereoselectivity with enol ethers substituted with aromatic groups. When a dibenzylester thymine cyclopropane was used with enol ether 21, the yield reached $95 \%$ (product $\mathbf{2 4 c}$ ). The styrenyl carbonucleoside $\mathbf{2 4 e}$ was isolated with 13:1 diastereoselectivity in 55\% yield. One more stereocenter is formed using a tri-substituted enol ether and the highly substituted nucleoside analogue $\mathbf{2 4 f}$ was obtained as a single diastereoisomer in $79 \%$ yield.

The annulation reaction was also conducted with 5-fluoro uracil cyclopropane 13c and uracil cyclopropane 13b under the conditions optimized for thymine cyclopropane 13a (Scheme 8, D). For the deprotection of the benzoyl group on the 5-fluorouracil nucleoside, we used again $\mathrm{NH}_{4} \mathrm{OH}$ in $\mathrm{EtOH}$, and the mixture was stirred at room temperature for 18 hours. Nucleoside analogues synthesized from benzaldehyde bearing uracil and 5-fluoro-uracil nucleobases 26a and 26b were obtained in $62 \%$ and $72 \%$ yield. With acetophenone, the yields remain similar, $76 \%$ and $71 \%$ for $\mathbf{2 6 c}$ and $\mathbf{2 6 d}$, respectively. Finally, carbonucleoside derivatives $\mathbf{2 6 e}$ and 26f were isolated in moderate to high yields $(81 \%$ and $51 \%$ respectively). 


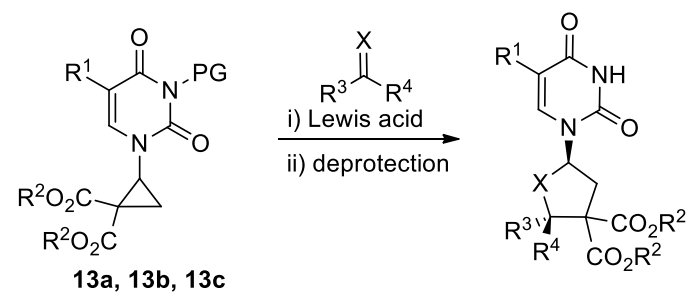

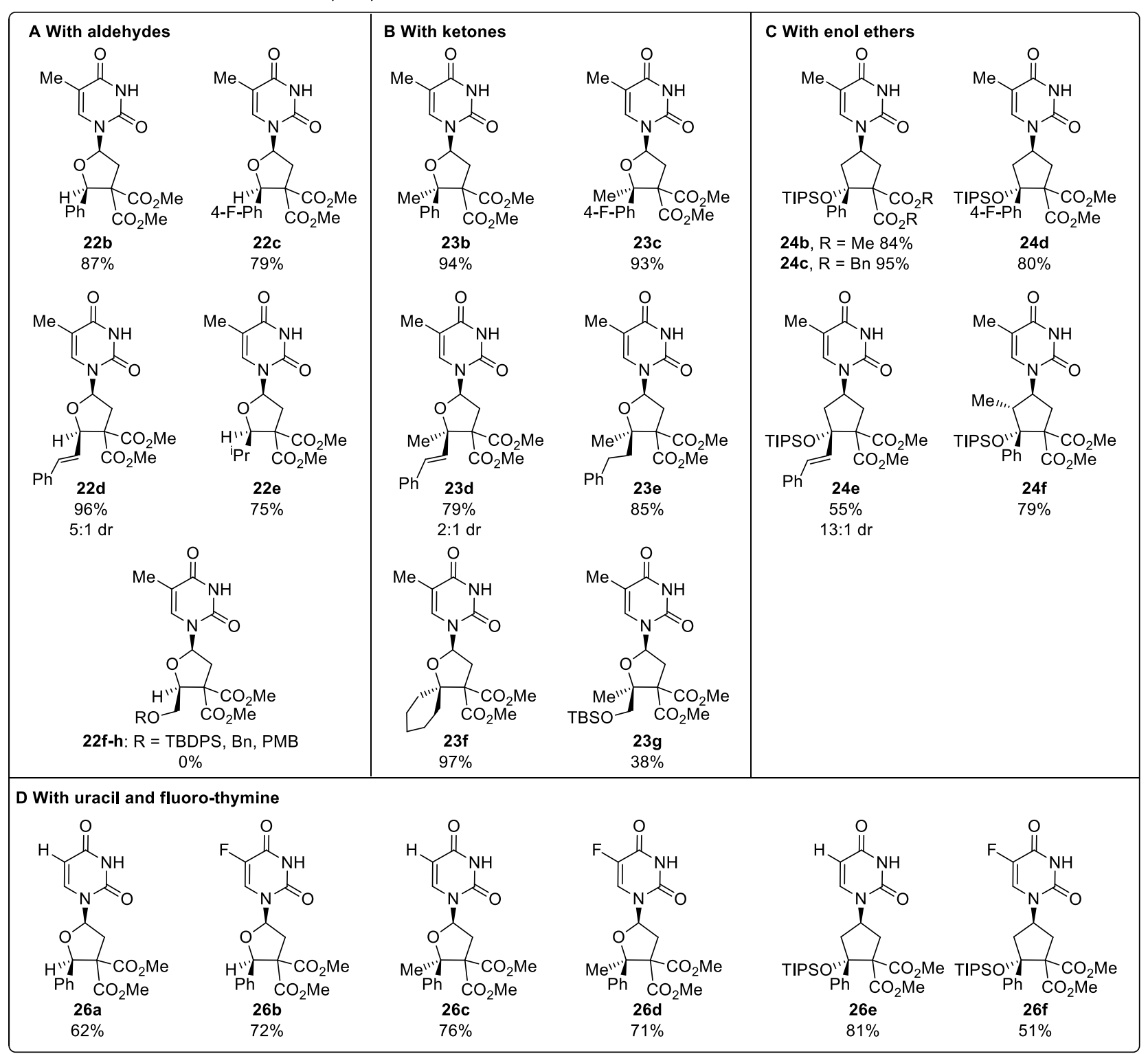

Scheme 8. Scope of the [3+2] annulation. The products were obtained as a single diastereoisomer, unless stated otherwise.

We then examined the scope of the $(4+2)$ annulation with thymine and 5-fluoro uracil cyclobutanes 18a and $\mathbf{1 8 b}$ and aldehydes (Scheme 9). The (4+2) annulation worked with electron neutral, benzaldehyde, electron poor 4-fluoro-benzaldehyde and thiophene-2carbaldehyde, providing pure compounds $25 \mathbf{a}, \mathbf{2 5 b}$ and 25c in 70 to $79 \%$ yield and $7: 1$ to $>20: 1$ diastereoselectivity after column chromatography and recrystallization to remove the benzamide side product.
Aldehydes bearing electron rich aromatic groups, such as meta-methoxy benzaldehyde and para-methoxy benzaldehyde, gave the corresponding nucleosides 25d and 25e in $76 \%$ yield. Styryl-substituted product $\mathbf{2 5 f}$ could also be obtained, but with a loss of stereoselectivity as previously noticed in the [3+2] reaction. 5-Fluoro-uracil cyclobutane $\mathbf{1 8 b}$, efficiently provided the desired nucleoside analogue 27 with good diastereoselectivity. 


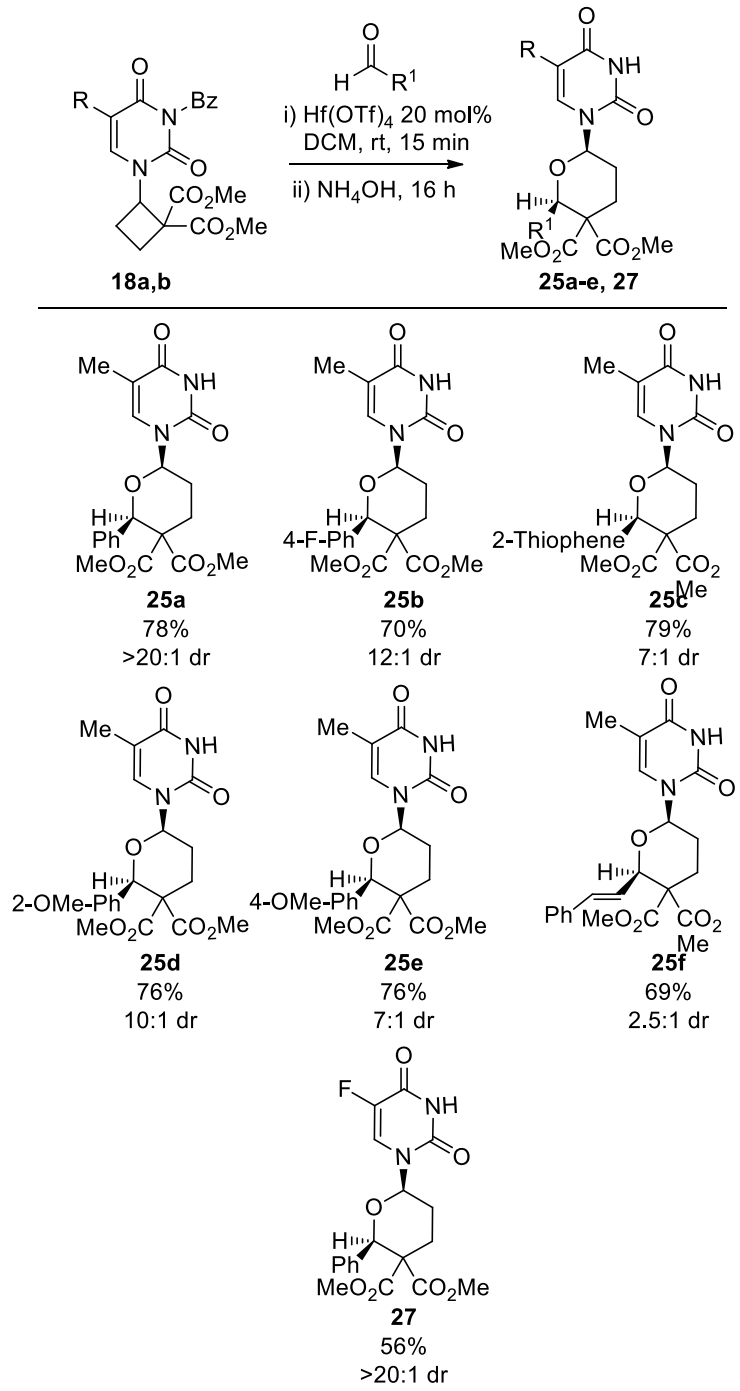

Scheme 9. Scope of the [4+2] annulation. Yield and diastereoselectivity are given after column chromatography and recrystallization.

In order to increase the diversity of our compounds and to provide potentially bioactive molecules, methods to access the hydroxymethyl substituted derivatives were examined. As discussed above, only $\alpha$-hydroxylated ketones could be used directly in the annulation reaction. However, TBS deprotection of product $\mathbf{2 3 g}$ led to a complex mixture containing a major compound tentatively identified as lactone $\mathbf{2 8},{ }^{[14]}$ and the desired free alcohol could not be accessed (Scheme 10).
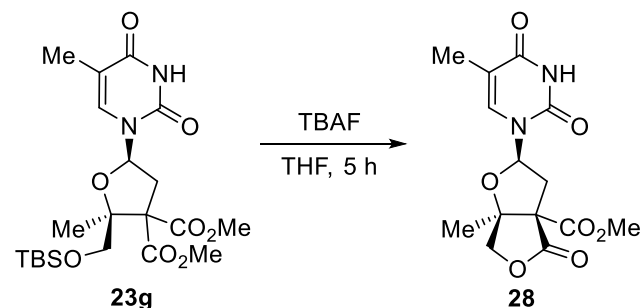

Tentative assignment
Scheme 10. Attempted deprotection of nucleoside analogue $\mathbf{2 3 g}$.

Consequently, we investigated modification of the esters to access the desired hydroxy methyl group. The Krapcho decarboxylation ${ }^{[15 a, b]}$ was first examined. With different salts $\left(\mathrm{NaCl}, \mathrm{LiCl}, \mathrm{LiI}\right.$ and $\left.\mathrm{Li}_{2} \mathrm{SO}_{4}\right)$, and DMF or DMSO as solvent at high temperature (from $80{ }^{\circ} \mathrm{C}$ to $130{ }^{\circ} \mathrm{C}$ ), no mono ester derivatives was isolated. Alternative methods for ester hydrolysis $\left(\mathrm{Ba}(\mathrm{OH})_{2}{ }^{[15 \mathrm{c}]} \mathrm{Me}_{3} \mathrm{SnOH}^{[15 \mathrm{~d}]}\right)$ or reduction (DiBAl-H, $\mathrm{LiAlH}_{4}, \mathrm{Me}(\mathrm{EtO})_{2} \mathrm{SiH}$ with $\left.\mathrm{Zn}(\mathrm{OAc})_{2}{ }^{[15 \mathrm{e}]}\right)$ were then investigated. Unfortunately, either no reaction was observed or degradation occurred. However, in $\mathrm{MeOH}$ at $70{ }^{\circ} \mathrm{C}$ with an excess $\mathrm{KOH}$, the compound $\mathbf{2 3 b}$ underwent saponification of both dimethylesters, followed by a diastereoselective decarboxylation, providing cleanly the carboxylic acid derivative 29 in high yield and diastereoselectivity (Scheme 11, A).
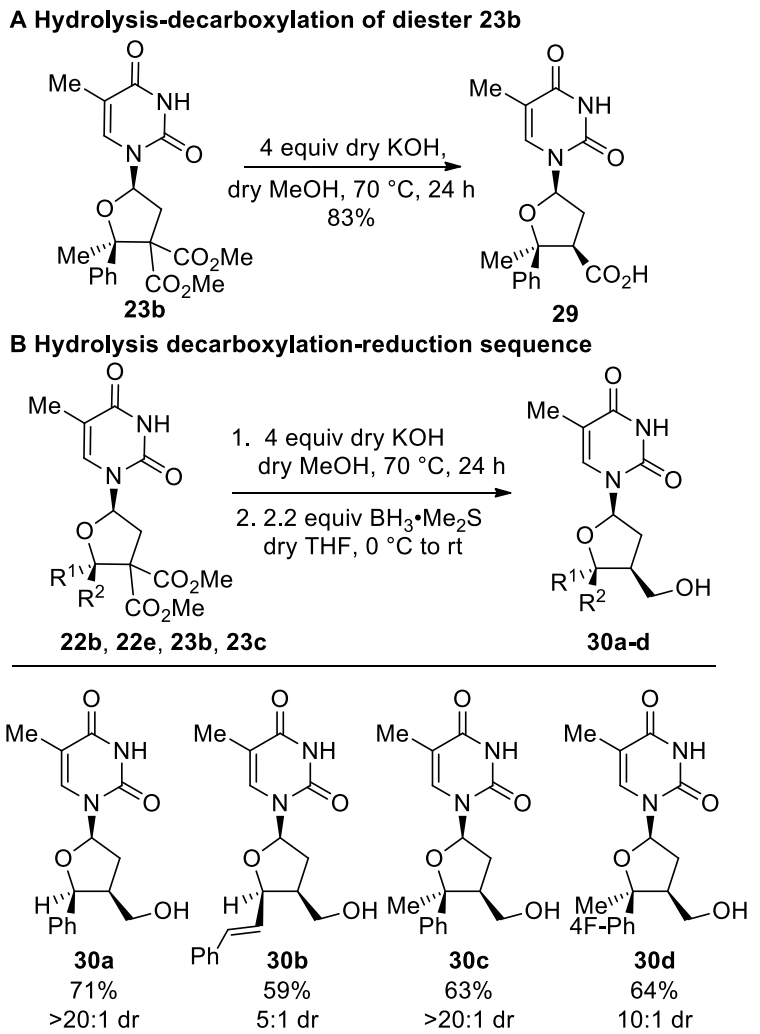

Scheme 11. Synthesis of hydroxymethyl nucleoside analogues.

The use of four equivalents fine powdered and dry $\mathrm{KOH}$ in combination with dry $\mathrm{MeOH}$ was important to obtain reproducibly high yields and selectivity. Reduction of the carboxylic acid with boron dimethylsulfide complex gave then access to 4'hydroxymethyl nucleoside derivatives. This efficient method was applied to the synthesis of nucleoside analogues 30a-d in good yield, but with lower diastereoselectivity for $\mathbf{3 0 b}$ and 30d (Scheme 11, B). 
When carbonucleoside derivatives were submitted to the saponification decarboxylation reaction, a mixture of unsaturated five membered rings was obtained due to silanol elimination. Consequently, we turned our attention to benzyl diester $\mathbf{2 4 c}$, which can be converted to the diacids under milder, non-basic conditions. The benzyl protecting groups were smoothly removed under hydrogenolysis conditions, then the diacid obtained was heated neat at $80{ }^{\circ} \mathrm{C}$ to allow decarboxylation to proceed providing the mono-acid 31 (Scheme 12). In this case, a controlled elimination of the silanol was observed. Finally, olefin hydrogenation followed by reduction of the carboxylic acid and protection of the corresponding alcohol afford the protected hydroxymethyl derivatives $\mathbf{3 2}$ in an efficient manner. Protection prior to isolation was preferred, as the free alcohol was unstable.

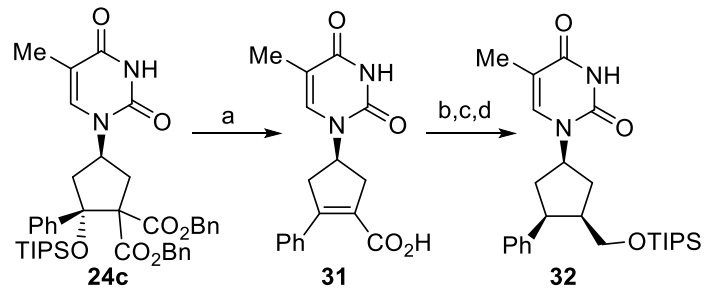

Scheme 12. Preparation of hydroxymethyl carbonucleoside derivative 32. a) $10 \% \mathrm{Pd} / \mathrm{C}, 1 \mathrm{~atm} \mathrm{H}_{2}$, $\mathrm{EtOH}, 57^{\circ} \mathrm{C}$, then neat, $80^{\circ} \mathrm{C}, 64 \%$. b) $5 \% \mathrm{Pd} / \mathrm{C}, 1 \mathrm{~atm}$ $\mathrm{H}_{2}, \mathrm{EtOH}, 85 \%$. c) $\mathrm{BH}_{3} \cdot \mathrm{SMe}_{2}$, THF. d) TIPSCl, DMF, imidazole, $55 \%$ over two steps.

We were also interested in the synthesis of 4'hydroxymethyl nucleoside analogues. Thus, we investigated the transformation of the styrene group of compounds $\mathbf{2 2 b}, \mathbf{2 4 b}$, and $\mathbf{3 0 b}$ into the corresponding aldehyde, which could lead after reduction, to the targeted alcohol (Scheme 13). Ozonolysis generally led to degradation of the starting materials. Dihydroxylation followed by oxidative cleavage, using several reported procedures (cat. $\mathrm{OsO}_{4}$ with $\mathrm{NaIO}_{4}$ and lutidine, cat. $\mathrm{OsO}_{4}$ with PIDA and lutidine, ${ }^{[16 a]}$ cat. $\mathrm{OsO}_{4}$ with $\mathrm{NMO}$ and citric acid followed by $\mathrm{NaIO}_{4}{ }^{[16 \mathrm{~b}]}$ ) was also not successful, leading to inseparable mixtures of unidentified products along with decomposition of the starting materials. ${ }^{[17]}$

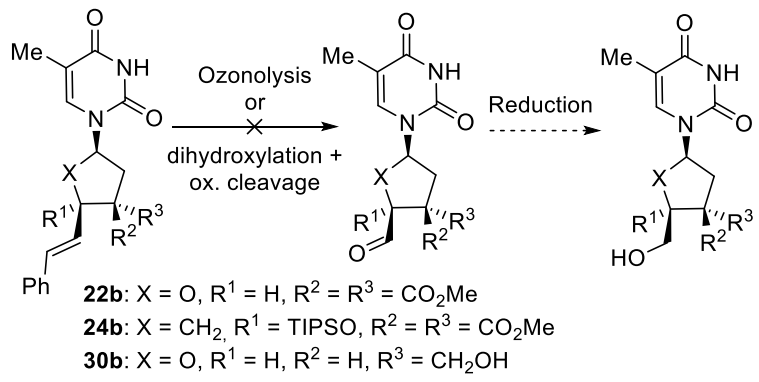

Scheme 13. Attempted synthesis of 4'-hydroxymethyl nucleoside analogues.
After the synthesis of pyrimidine nucleoside analogues had been achieved via annulation reactions of strained rings, we turned our attention to purine nucleoside analogues. As substituents on the cyclopropane, we chose adenine, 6-chloro-2-amino purine and 6-chloro purine. 6-Chloro-2-amino purine derivatives are interesting, as they can be used as precursors for both adenine and guanine derivatives. ${ }^{[18]}$

In order to synthesize purine cyclopropanes, we firstly thought about using the protocol developed for the pyrimidines. However, the palladium catalyzed vinylation was not successful with purines. As an alternative approach, a tandem Michael addition/cyclopropane formation of purines on chloromethyl-ethylidene malonates was envisaged. Indeed, Geen and co-workers had reported a single example of synthesis of a 6-chloro-2-amino purine derived diester cyclopropane using this approach. ${ }^{[19]}$

As described by Lehnert, chloromethyl-ethylidene malonates can be prepared via a Knoevenagel condensation of chloroacetaldehyde on malonate derivatives in presence of two equivalents of titanium tetrachloride and four of pyridine. ${ }^{[20]}$ Unfortunately, in our hand this reaction turned out to give the product in highly fluctuating yields (from $60 \%$ to $0 \%$ ), as polymerization of the starting material was often observed. Deshmuck and co-workers have described the condensation of trichloroacetaldehyde on diethylmalonate using Montmorillonite $\mathrm{K}-10$ in $\mathrm{Ac}_{2} \mathrm{O}$ at $150{ }^{\circ} \mathrm{C}$ for five hours. ${ }^{[21]}$ These conditions were then examined with chloroacetaldehyde 34 , but after only 10 minutes of reaction, complete degradation was observed. When the temperature was lowered to $110{ }^{\circ} \mathrm{C}$ complete conversion was obtained after $1 \mathrm{~h}$ to the desired product 35 , together with the aldol dimer 36 of chloroacetaldehyde. As the two compounds were difficult to separate, the reaction conditions were optimized in order to avoid dimer formation. At $70{ }^{\circ} \mathrm{C}$ for 7 hours, the reaction was complete giving access to the desired ethylidene malonate $\mathbf{3 5}$ along with some remaining malonate $\mathbf{3 3}$, which was easily removed by column chromatography (Scheme 14). The product was isolated up to $61 \%$ on a five gram scale reaction.

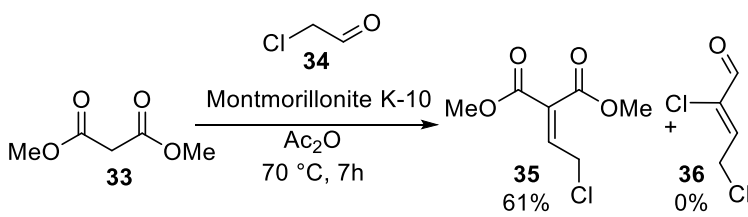

Scheme 14. Preparation of chloromethyl-ethylidene dimethylmalonate (35).

Furthermore, chloromethyl-ethylidene malonate $\mathbf{3 7}$ bearing pentyl esters was synthesized in order to increase the solubility of the purine cyclopropanes in organic solvents. It was obtained from the malonic acid through esterification with the corresponding pentyl 
alcohol followed by the optimized Knoevenagel reaction.

The obtained alkylidene malonates $\mathbf{3 5}$ and $\mathbf{3 7}$ were then submitted to the previously reported conditions for cyclopropanation (Scheme 15). ${ }^{[19]}$ Purine cyclopropanes were obtained in moderate to excellent yields, always with complete regioselectivity. Generally, cyclopropanes with geminal dimethyl ester 38a, 38b and 38c were obtained in lower yields. Purification was a major issue due to their poor solubility in organic solvents and to their high polarity. The other three cyclopropanes 38d, 38e and 38f with pentyl ester chains were isolated in higher yields (62 to $93 \%$ ), as they were well soluble in organic solvents.

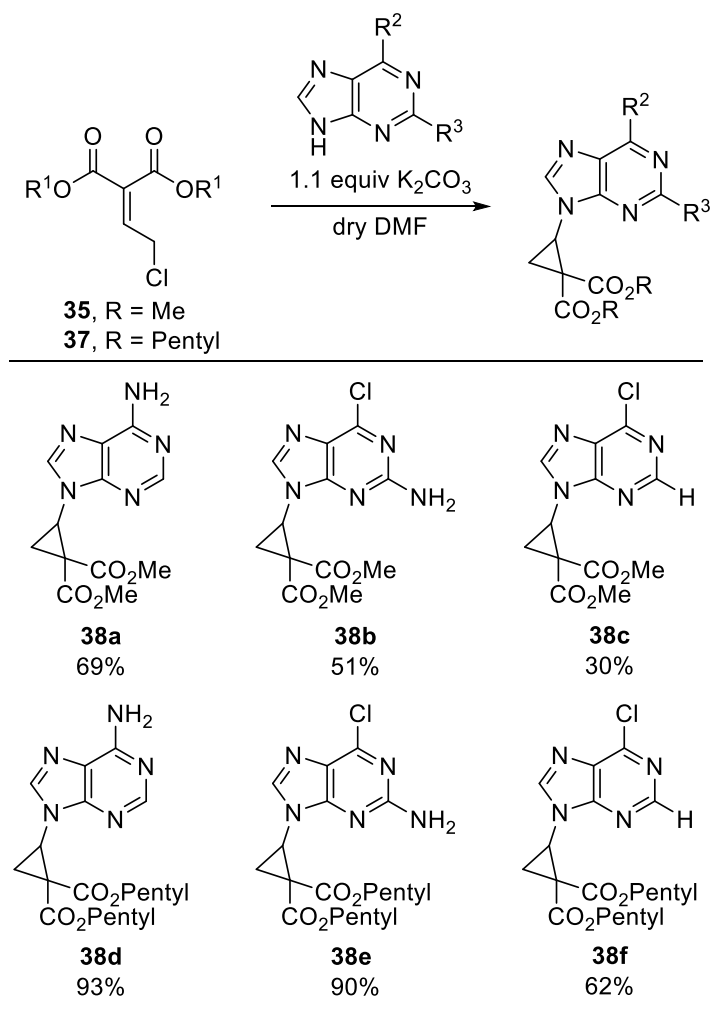

Scheme 15. Synthesis of purine cyclopropanes.

The solubility of dimethyl ester cyclopropanes 38a-c was poor in all common organic solvents, even if the 6-chloropurine cyclopropane 38c exhibited a slightly improved solubility. Only partial solubility in hot acetonitrile or DMF was observed. In contrast, the purine cyclopropanes 38d-f bearing pentyl esters were completely soluble in dichloromethane at room temperature. In order to prevent any potential catalyst deactivation or side-reaction and also improve its solubility, the free amine of the 6-chloro-amino-purine cyclopropane $\mathbf{3 8 b}$ was protected with two tertbutoxycarbonyl groups to give product 39 (Scheme 16). The yield of the protection step is highly dependent on the solvent: In THF or DMSO, the isolated yields were typically around $40 \%$, whereas in acetonitrile the product was obtained in $85 \%$ yield.

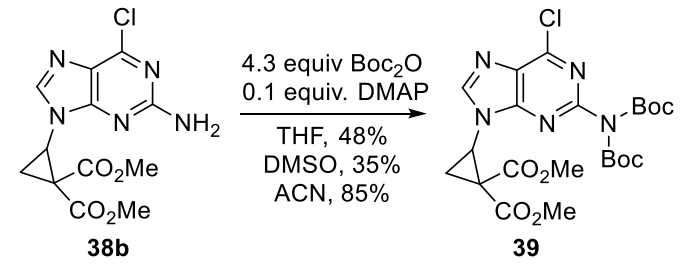

Scheme 16. Preparation of the protected purine cyclopropane.

Attempts to protect the free amine with a benzyl group via a $S_{N}^{2}$ reaction using a phase transfer reagent ${ }^{[22]}$ or through reductive amination ${ }^{[23]}$ failed, as did protection with dimethylcarbonate.

Cyclopropanes 38a-f were then examined in the $(3+2)$ annulation with benzaldehyde (19) as partner and a broad range of Lewis acids. The first cyclopropane tested was the adenine cyclopropane 38a bearing methyl esters. The screening was initially planned in dichloromethane, which had been the solvent of choice for the $(3+2)$ annulation of thymine-substituted cyclopropanes. Unfortunately, adenine cyclopropane 38a displayed extremely poor solubility in DCM, THF and ACN. Even with slightly more soluble purine cyclopropanes $\mathbf{3 8 b}$ no reaction was observed using $\mathrm{In}(\mathrm{OTf})_{3}$ as catalyst in several solvents (DCM, DCE, $\mathrm{DCM} / \mathrm{MeOH}, \mathrm{CCl}_{4}$, THF, ACN, toluene, $\mathrm{MeOH}$, benzene, DMF and DMSO). We hypothesized that the lack of reactivity of this cyclopropane may be the consequence of catalyst poisoning due to the coordination of the Lewis acid to the free amine of the substrate. Therefore, the annulation of 38c was examined with different Lewis acids $\left(\mathrm{Zn}(\mathrm{OTf})_{2}, \mathrm{AuCl}_{3}\right.$, $\mathrm{Sn}(\mathrm{OTf})_{2}, \quad \mathrm{Sc}(\mathrm{OTf})_{3}, \quad \mathrm{Hf}(\mathrm{OTf})_{4}, \quad \mathrm{In}(\mathrm{OTf})_{3}$, stoichiometric $\operatorname{In}(\mathrm{OTf})_{3}, \mathrm{Cu}(\mathrm{OTf})_{2}, \mathrm{FeCl}_{3} \bullet \mathrm{Al}_{2} \mathrm{O}_{3}$ and $\left.\mathrm{Yb}(\mathrm{OTf})_{3}\right)$. However, also in this case, no conversion to the desired nucleoside analogues was observed.

No reaction was observed with pentyl esterssubstituted adenine cyclopropane $\mathbf{3 8 d}$ as well as the other purines cyclopropane 38e and 38f, even though the solubility in DCM at room temperature was high and a wide range of Lewis acids were examined $\left(\mathrm{Zn}(\mathrm{OTf})_{2}, \mathrm{FeCl}_{3}, \mathrm{AuCl}_{3}, \mathrm{AuCl}, \mathrm{Sn}(\mathrm{OTf})_{2}, \mathrm{Sc}(\mathrm{OTf})_{3}\right.$, $\left.\mathrm{Hf}(\mathrm{OTf})_{4}, \operatorname{In}(\mathrm{OTf})_{3}, \mathrm{Cu}(\mathrm{OTf})_{2}, \mathrm{Yb}(\mathrm{OTf})_{3}\right)$. Another possibility for the lack of reactivity observed, may be the hindrance created by the pentyl side chains around the esters avoiding the catalyst to chelate the malonate and therefore the reaction to proceed. However, when the soluble protected 6-chloro-2-amino purine dimethylester cyclopropane $\mathbf{3 9}$ was submitted to the conditions for $(3+2)$ annulation with indium triflate as catalyst in either DCM or THF, only removal of the tert-butoxycarbonyl was observed.

To completely exclude catalyst poisoning by the substrate, we performed a competition experiment between pyrimidine cyclopropane $13 \mathbf{c}$ and purine analogue 38e under the conditions for $(3+2)$ annulation (Scheme 17). After 45 minutes of reaction, complete 
conversion of the pyrimidine cyclopropane 13c into the corresponding nucleoside analogue $\mathbf{4 0}$ was observed, while the purine cyclopropane 38e stayed untouched.

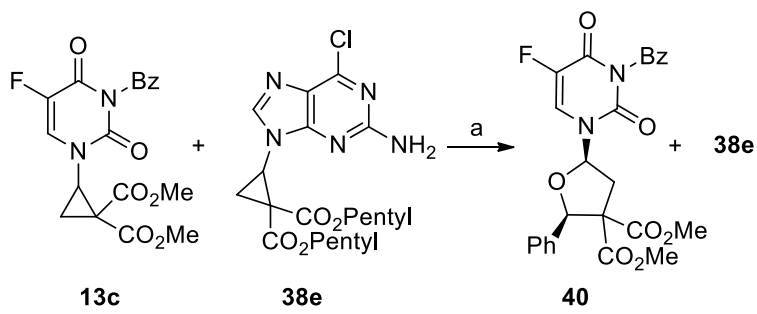

Scheme 17. Competition experiment. a) 0.2 equiv $\ln (\mathrm{OTf})_{3}, 2.4$ equiv benzaldehyde (19), DCM.

From this result, we can conclude that purine cyclopropanes 38a-f do not coordinate too strongly to the Lewis acid. However, purine cyclopropanes 38a-f are not suitable substrates for the $(3+2)$ annulation, probably due to their different electronic properties leading to too low reactivity. In the future, further modulation of the DA cyclopropane structure will be required to extend the developed annulation processes to this important class of substrates.

\section{Conclusions}

A short synthesis of pyrimidine DA cyclopropanes and cyclobutanes was designed based on a regioselective vinylation followed by cyclopropanation or $(2+2)$ cycloaddition. Purine DA cyclopropanes were prepared from chloromethyl-ethylidene malonates via a tandem Michael addition intramolecular substitution sequence. Pyrimidine nucleosides analogues were accessed from the DA cyclopropanes and DA cyclobutanes via $(3+2)$ and $(4+2)$ annulation reactions, opening a new chemical space for further biological investigations. Unfortunately, purine DA cyclopropanes remain unreactive in the $(3+2)$ annulation reactions.

\section{Acknowledgements}

The NCCR chemical biology of the Swiss National Science Foundation is acknowledged for financial support.

\section{References}

[1] a) Modified Nucleosides in Biochemistry, Biotechnology and Medicine, P. Herdewijn ed., Wiley- $\mathrm{VCH}$,

Weinheim, 2008; b) S. Broder, Antiviral Res. 2010, 85, 1; c) J. C. Martin, M. J. M. Hitchcock, E. De Clercq, W. H. Prusoff, Antiviral Res. 2010, 85, 34; d) T. Cihlar, A. S. Ray, Antiviral Res. 2010, 85, 39; e) G. Antonelli, O.
Turriziani, Int. J. Antimicrob. Agents 2012, 40, 95; f) L. P. Jordheim, D. Durantel, F. Zoulim, C. Dumontet, Nat. Rev. Drug Discovery 2013, 12, 447.

[2] a) U. Niedball, H. Vorbrüggen, Angew. Chem. Int. Ed. 1970, 9, 461; b) G. Shaw, R. N. Warrener, M. H. Maguire, R. K. Ralph, J. Chem. Soc. 1958, 2294; Reviews: c) M. T. Crimmins, Tetrahedron 1998, 54, 9229; d) H. Vorbrüggen, C. Ruh-Polenz, Handbook of Nucleoside Synthesis, John Wiley \& Sons, 2001; c) Antiviral Nucleosides: Chiral Synthesis and Chemotherapy, C. K. Chu ed., Elsevier, Amsterdam, 2003; d) C. Mathe, C. Perigaud, Eur. J. Org. Chem. 2008, 1489; e) G. Romeo, U. Chiacchio, A. Corsaro, P. Merino, Chem. Rev. 2010, 110, 3337; f) Chemical Synthesis of Nucleoside Analogues, P. Merino ed., John Wiley \& Sons, Hoboken, New Jersey, 2013; g) O. Boutureira, M. I. Matheu, Y. Diaz, S. Castillon, Chem. Soc. Rev. 2013, 42, 5056; h) L. Scagnelli, M. G. Memeo, S. Carosso, B. Bovio, P. Quadrelli, Eur. J. Org. Chem. 2013, 3835.

[3] a) S. Daluge, R. Vince, Tetrahedron Lett. 1976, 17, 3005; b) R. Singh, R. Vince, Chem. Rev. 2012, 112, 4642; c) J. Velcicky, A. Lanver, J. Lex, A. Prokop, T. Wieder, H. G. Schmalz, Chem. Eur. J. 2004, 10, 5087; d) W. J. Choi, J. G. Park, S. J. Yoo, H. O. Kim, H. R. Moon, M. W. Chun, Y. H. Jung, L. S. Jeong, J. Org. Chem 2001, 66, 6490; e) B. M. Trost, G. H. Kuo, T. Benneche, J. Am. Chem. Soc. 1988, 110, 621; f) B. M. Trost, L. S. Kallander, J. Org. Chem. 1999, 64, 5427; g) L. F. Tietze, C. Stadler, N. Böhnke, G. Brasche, A. Grube, Synlett 2007, 485; h) M. J. Mulvihill, J. L. Gage, M. J. Miller, J. Org. Chem 1998, 63, 3357; i) G. A. Boyle, C. D. Edlin, Y. F. Li, D. C. Liotta, G. L. Morgans, C. C. Musonda, Org. Biomol. Chem. 2012, $10,1870$.

[4] a) H. U. Reissig, R. Zimmer, Chem. Rev 2003, 103, 1151; b) M. Yu, B. L. Pagenkopf, Tetrahedron 2005, 61,321 ; c) C. A. Carson, M. A. Kerr, Chem. Soc. Rev 2009, 38, 3051; d) F. De Simone, J. Waser, Synthesis 2009, 3353; f) T. F. Schneider, J. Kaschel, D. B. Werz, Angew. Chem. Int. Ed. 2014, 53, 5504.

[5] [3+2] Annulation with imido-cyclopropanes: a) F. de Nanteuil, J. Waser, Angew. Chem. Int. Ed. 2011, 50, 12075 ; b) F. Benfatti, F. de Nanteuil, J. Waser, Chem. Eur. J. 2012, 18, 4844; c) F. Benfatti, F. de Nanteuil, J. Waser, Org. Lett. 2012, 14, 386; d) F. de Nanteuil, E. Serrano, D. Perrotta, J. Waser, J. Am. Chem. Soc. 2014, 136, 6239; e) E. Serrano, F. de Nanteuil, J. Waser, Synlett 2014, 25, 2285; Other reactions with imidocyclopropanes: f) F. de Nanteuil, J. Loup, J. Waser, Org. Lett. 2013, 15, 3738; g) R. Tejero, A. Ponce, J. Adrio, J. C. Carretero, Chem. Commun. 2013, 49, 10406; h) A. R. Rivero, I. Fernandez, M. A. Sierra, Org. Lett. 2013, 15, 4928; [4+2] Annulations with imidocyclobutanes: i) F. de Nanteuil, J. Waser, Angew. Chem. Int. Ed. 2013, 52, 9009; For a review, see: j) F. de Nanteuil, F. De Simone, R. Frei, F. Benfatti, E. Serrano, J. Waser, Chem. Commun. 2014, 50, 10912.

[6] a) S. Racine, F. de Nanteuil, E. Serrano, J. Waser, Angew. Chem. Int. Ed. 2014, 53, 8484; b) D. Perrotta, S. Racine, J. Vuilleumier, F. de Nanteuil, J. Waser, Org. Lett. 2015, 17, 1030.

[7] a) P. Ciapetti, M. Taddei, Tetrahedron 1998, 54, 11305; b) H.-J. Gi, Y. Xiang, R. F. Schinazi, K. Zhao, J. Org. Chem. 1997, 62, 88.

[8] P. Arsenyan, A. Petrenko, E. Paegle, S. Belyakov, Mendeleev Commun. 2011, 21, 326. 
[9] K. S. Toti, M. Derudas, C. McGuigan, J. Balzarini, S. Van Calenbergh, Eur. J. Med. Chem. 2011, 46, 3704.

[10] B. Nawrot, O. Michalak, S. Olejniczak, M. Wieczorek, T. Lis, W. Stec, Tetrahedron 2001, 57, 3979.

[11] a) C. G. Espino, K. W. Fiori, M. Kim, J. Du Bois, J. Am. Chem. Soc. 2004, 126, 15378; b) F. GonzalezBobes, M. D. B. Fenster, S. Kiau, L. Kolla, S. Kolotuchin, M. Soumeillant, Adv. Synth. Catal. 2008 $350,813$.

[12] Yield calculated from diazomalonate. If calculated with uracil as limiting reagent: 13b: 33\%, 14: $38 \%$.

[13] a) T. Kametani, K. Kigasawa, M. Hiiragi, K. Wakisaka, K. Nakazato, K. Ichikawa, K. Fukawa, O. Irino, N. Nishimura, T. Okada, J. Med. Chem. 1982, 25, 1219; b) J. Yamashita, M. Yasumoto, S. Takeda, H. Matsumoto, N. Unemi, J. Med. Chem. 1989, 32, 136; c) G. Y. Song, V. Paul, H. Choo, J. Morrey, R. W. Sidwell, R. F. Schinazi, C. K. Chu, J. Med. Chem. 2001, 44, 3985.

[14] NMR data of compound $\mathbf{2 8}$ corresponded to the proposed structure, however this compound could not be purified for complete characterization.

[15] a) A. P. Krapcho, G. A. Glynn, B. J. Grenon, Tetrahedron Lett. 1967, 8, 215; b) A. P. Krapcho, E. G. E. Jahngen, A. J. Lovey, F. W. Short, Tetrahedron Lett. 1974, 15, 1091; c) N. Pérez-Hernández, M. Febles, C. Pérez, R. Pérez, M. L. Rodríguez, C. Foces-Foces, J. D. Martín, J. Org. Chem. 2006, 71, 1139; d) K. C.
Nicolaou, A. A. Estrada, M. Zak, S. H. Lee, B. S. Safina, Angew. Chem. Int. Ed. 2005, 44, 1378; e) S Das, K. Möller, K. Junge, M. Beller, Chem. Eur. J. 2011, 17,7414 .

[16] a) K. C. Nicolaou, V. A. Adsool, C. R. H. Hale, Org. Lett. 2010, 12, 1552; b) P. Dupau, R. Epple, A. Thomas, V. Fokin, K. Barr. Sharpless, Adv. Synth. Catal. 2002, $344,421$.

[17] Attempts to directly reduce the crude product in one-pot were not successful.

[18] L. Beigelman, P. Haeberli, D. Sweedler, A. Karpeisky, Tetrahedron 2000, 56, 1047.

[19] G. R. Geen, P. M. Kincey, B. M. Choudary, Tetrahedron Lett. 1992, 33, 4609.

[20] W. Lehnert, Tetrahedron 1973, 29, 635.

[21] A. R. A. S. Deshmuck, D. G. Panse, B. M. Bhawal, Synth. Commun. 1999, 10, 1801.

[22] A. R. Maguire, W.-D. Meng, S. M. Roberts, A. J. Willetts, J. Chem. Soc. Perkin 1 1993, 1795.

[23] BRISTOL-MYERS SQUIBB COMPANY, Patent: WO2003/99286 A1, 2003; Location in patent: P.89.

Received: ((will be filled in by the editorial staff))

Accepted: ((will be filled in by the editorial staff))

Published online: ((will be filled in by the editorial staff)) 
Graphical Abstract:

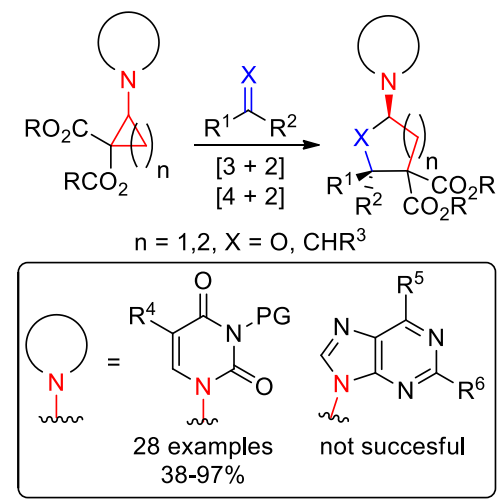




\section{Contents}

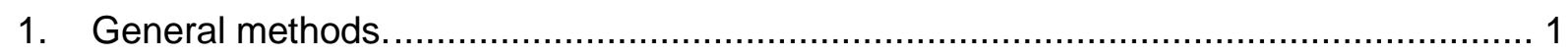

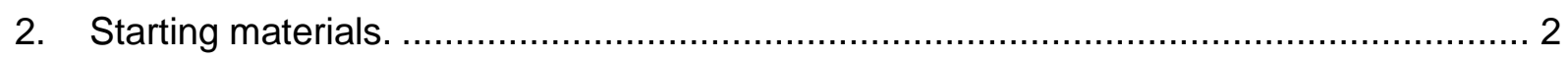

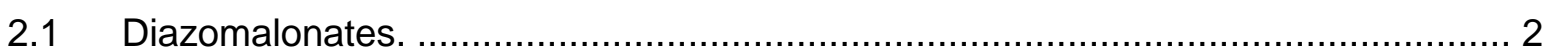

2.2 Methylidene and ethylidene malonates........................................................... 2

2.3 Pyrimidine cyclopropanes......................................................................... 4

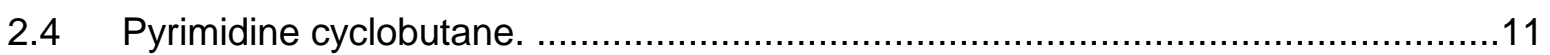

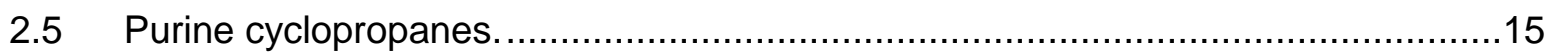

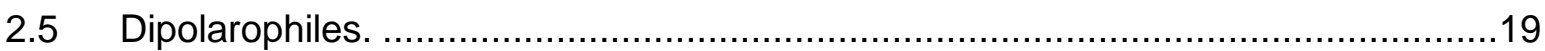

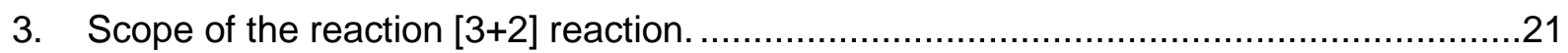

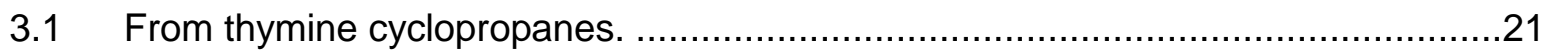

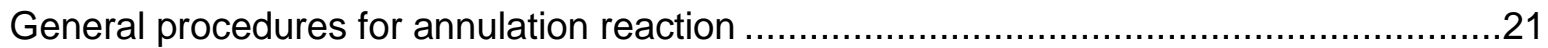

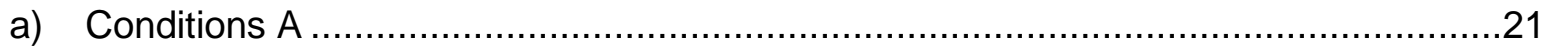

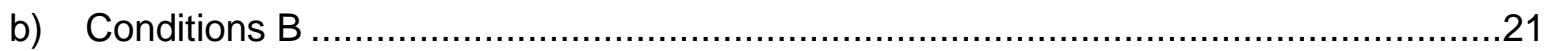

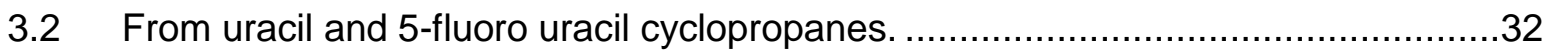

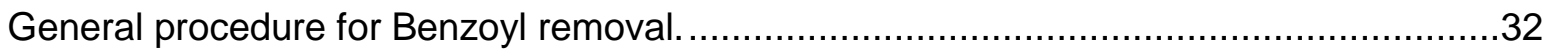

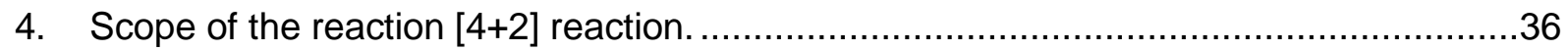

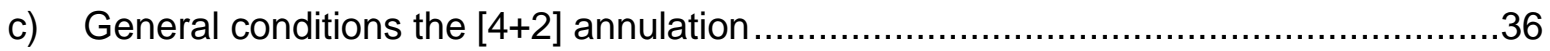

6. Thymine based nucleoside analogues derivatizations. ............................................41

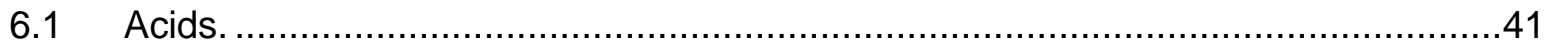

General procedure for hydrolysis and decarboxylation reaction ..................................41

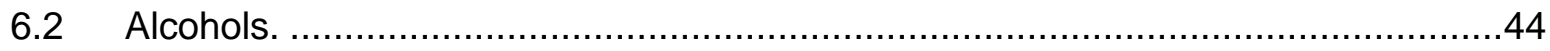

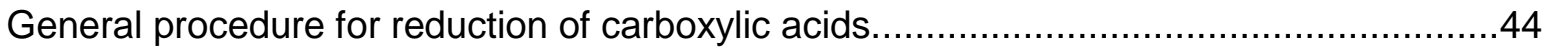

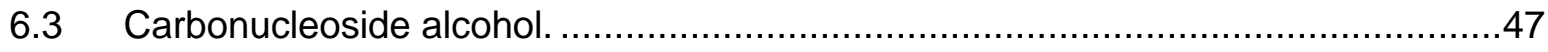

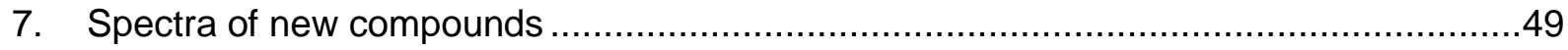




\section{General methods.}

All reactions were carried out in flamed-dried glassware under an atmosphere of nitrogen, unless stated otherwise. HPLC grade solvents purchased from Sigma-Aldrich or freshly distilled solvents were used for flash chromatography. Reaction solvents were dried by passage over activated alumina under nitrogen atmosphere $\left(\mathrm{H}_{2} \mathrm{O}\right.$ content $<30 \mathrm{ppm}$, KarlFischer titration). Commercially available reagents were purchased from Acros, Aldrich, Fluka, VWR, Aplichem, Merck or TCl and used without any further purification. Chromatographic purification was performed as flash chromatography using Macherey-Nagel silica 40-63, $60 \AA$, using the solvents indicated as eluent with 0.1-0.5 bar pressure. TLC was performed on Merck silica gel 60 F254 TLC plates and visualized with UV light and permanganate stain. Melting points were measured on a calibrated Büchi B-540 melting point apparatus using open glass capillaries. ${ }^{1} \mathrm{H}$ NMR spectra were measured on a Brucker DPX-400, $400 \mathrm{MHz}$ spectrometer, all signals are reported in ppm with the corresponding internal solvent peak or TMS as standard. The data is being reported as $(s=$ singlet, $d=$ doublet, $t=$ triplet, $q=$ quadruplet, $q i$ = quintet, $\mathrm{m}=$ multiplet or unresolved, $\mathrm{br}=$ broad signal, coupling constant(s) in Hz, integration; interpretation). ${ }^{13} \mathrm{C}$ NMR spectra were carried out with $1 \mathrm{H}$-decoupling on a Brucker DPX-400 $100 \mathrm{MHz}$. All signals are reported in ppm with the corresponding internal solvent signal or TMS as standard. Infrared spectra were obtained on a JASCO FT-IR B4100 spectrophotometer with an ATR PRO410-S and a ZnSe prisma and are reported as $\mathrm{cm}^{-1}(\mathrm{w}=$ weak, $\mathrm{m}=$ medium, $\mathrm{s}=$ strong, sh = shoulder). High resolution mass spectrometric measurements were performed by the mass spectrometry service of ISIC at the EPFL on a MICROMASS (ESI) Q-TOF Ultima API. All experimental procedures to synthesize the compounds described in the article are given. Only spectra of compounds which were not described in our previous publications are given. For compounds 10a-c, 12a-c, 13a-c, 14, 16a,b, 17, 18a,b, 22a-f, 23a-f, 24a-f, 25a-f, 26a-f, 27, 29, 30a-d, 31 and 32, please refer to the previous reports. ${ }^{1}$

1 a) S. Racine, F. de Nanteuil, E. Serrano, J. Waser, Angew. Chem. Int. Ed. 2014, 53, 8484-8487. b) D. Perrotta, S. Racine, J. Vuilleumier, F. de Nanteuil, J. Waser, Org. Lett. 2015, 17, 1030-1033. c) F. de Nanteuil, J. Waser, Angew. Chem. Int. Ed. 2013, 52, 9009-9013. 


\section{Starting materials.}

\subsection{Diazomalonates.}

\section{Dibenzyl 2-diazomalonate (42).}<smiles>CCNC(=O)OCc1ccccc1</smiles>

41<smiles>O=C(OCc1ccccc1)C(=[W])C(=O)OCc1ccccc1</smiles>

42

In flame dried flask under nitrogen, 4-acetamidobenzenesulfonyl azide $(1.27 \mathrm{~g}, 5.28 \mathrm{mmol}, 1.5$ equivuiv) was dissolved in acetonitrile $(15 \mathrm{~mL})$ and triethylamine $(1.17 \mathrm{~mL}, 8.44 \mathrm{mmol}, 2.4$ equivuiv) and dibenzyl malonate (41) $(0.88 \mathrm{~mL}, 3.5 \mathrm{mmol}$, 1 equivuiv) were added. The reaction mixture was stirred at room temperature for 2 days. The solvent was evaporated and the crude product was filtered on coton with acetonitrile $(30 \mathrm{~mL})$. The crude mixture was concentrated under reduced pressure and filtered on coton one more time with DCM (30 mL) and finally purified by column chromatography, eluting with pentane/AcOEt (9:1) and $1 \% \mathrm{NEt}_{3}$ mixture to obtain the pure diazo-compound $42(1.02 \mathrm{~g}, 3.29 \mathrm{mmol}, 93 \%)$ as a slightly yellow solid.

RF $($ AcOEt/Pent $(1: 9)=0.22$

Mp 54.8-55.4 $4^{\circ} \mathrm{C}$. (Decomposition)

${ }^{1} \mathrm{H}$ NMR $\left(400 \mathrm{MHz}\right.$, Chloroform-d) $\delta$ 7.39- $7.34(\mathrm{~m}, 10 \mathrm{H}, \mathrm{Ar}-\mathrm{H}), 5.28\left(\mathrm{~s}, 4 \mathrm{H}, \mathrm{CH}_{2}\right)$.

${ }^{13}$ C NMR (101 MHz, Chloroform-d) $\delta 160.8,135.3,128.7,128.5,128.3,67.1$.

One carbon is not resolved.

IR 3035 (w), 2141 (s), 1757 (s), 1689 (m), 1388 (s), 1271 (m), 1077 (s), 760 (s).

HRMS (ESI) calcd for $\mathrm{C}_{17} \mathrm{H}_{14} \mathrm{~N}_{2} \mathrm{NaO}_{4}{ }^{+}[\mathrm{M}+\mathrm{Na}]^{+} 333.0846$; found 333.0856.

Dimethyl 2-diazomalonate (44).

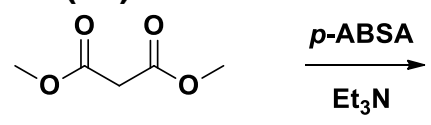

33<smiles>[CH]=[W]C(=O)C(=O)OC</smiles>

44

In a flame dried flask under nitrogen, 4-acetamidobenzenesulfonyl azide $(6.82 \mathrm{~g}, 28.4 \mathrm{mmol}$, 1.5 equiv) was dissolved in acetonitrile $(80 \mathrm{~mL})$ and triethylamine $(6.3 \mathrm{~mL}, 45 \mathrm{mmol}, 2.4$ equiv) and dimethyl malonate (33) $(2.2 \mathrm{~mL}, 19 \mathrm{mmol}, 1$ equiv) were added. The reaction mixture was stirred at room temperature for 1 day. The solvent was evaporated and the crude product was filtered on coton with acetonitrile $(30 \mathrm{~mL})$. The crude mixture was concentrated under reduced pressure and filtered on coton one more time with $\mathrm{DCM}(30 \mathrm{~mL})$ and finally purified by column chromatography, eluting with pentane/AcOEt (9:1) and $1 \% \mathrm{NEt}_{3}$ mixture to obtain the pure diazo-compound $44(2.67 \mathrm{~g}, 16.9 \mathrm{mmol}, 94 \%)$ as a slightly yellow oil (solid at $4{ }^{\circ} \mathrm{C}$ ).

${ }^{1} \mathrm{H}$ NMR $\left(400 \mathrm{MHz}\right.$, Chloroform-d) $\delta 3.84\left(\mathrm{~s}, 4 \mathrm{H}, \mathrm{CH}_{2}\right)$.

${ }^{1} \mathrm{H}$ NMR values correspond to the literature. ${ }^{2}$

\subsection{Methylidene and ethylidene malonates.}

\section{Dimethyl 2-methylenemalonate (15).}

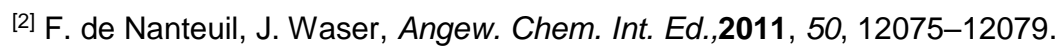




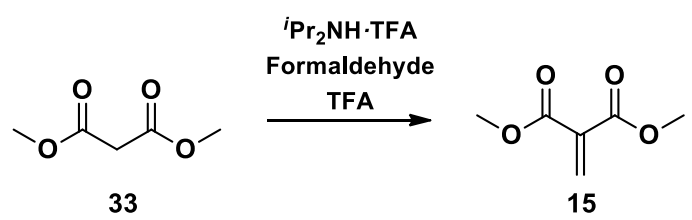

Following the described procedure of De Nanteuil et al., ${ }^{1 \mathrm{c}}$ paraformaldehyde $(2.7 \mathrm{~g}, 90 \mathrm{mmol}$, 2.0 equiv) and diisopropylamine 2,2,2-trifluoroacetate $(9.7 \mathrm{~g}, 45 \mathrm{mmol}, 1.0$ equiv) were added in a flame dried $250 \mathrm{~mL}$ round flask with a condenser. Then, tetrahydrofuran $(65 \mathrm{~mL})$, dimethyl malonate (33) $(5.1 \mathrm{~mL}, 45 \mathrm{mmol}, 1.0$ equiv) and trifluoroacetic acid $(0.35 \mathrm{~mL}, 4.5 \mathrm{mmol}, 0.1$ equiv) were added into the flask under nitrogen atmosphere and the suspension was stirred to reflux for 2 hours. Paraformaldehyde $(2.7 \mathrm{~g}, 90 \mathrm{mmol}, 2.0$ equiv) was added to the reaction mixture and the reflux was restarted for 12 hours. The reaction mixture was cooled to room temperature and THF was removed under reduced pressure. The crude product was dissolved in diethyl ether $(25 \mathrm{~mL})$ and filtrated trough cotton. The organic layer was washed two times with $1 \mathrm{M} \mathrm{HCl}(25 \mathrm{~mL})$, the aqueous layers were combined and washed three times with diethyl ether $(50 \mathrm{~mL})$. The combined organic layers were dry over magnesium sulfate, filtrated and concentrated under reduced pressure. The crude mixture was obtained as a colorless oil (15) $(2.5 \mathrm{~g})$.

${ }^{1} \mathrm{H}$ NMR (400 MHz, Chloroform-d) $\delta 6.58\left(\mathrm{~s}, 2 \mathrm{H}, \mathrm{CH}_{2}\right), 3.83\left(\mathrm{~s}, 6 \mathrm{H}\right.$, ester $\left.\mathrm{CH}_{3}\right)$.

${ }^{1} \mathrm{H}$ NMR values correspond to the literature. ${ }^{2}$

Dimethyl 2-(2-chloroethylidene)malonate (35).<smiles>COC(=O)C(=O)OCCOC(=O)C(=CCCl)C(=O)OC</smiles>

Following an adapted procedure from Deshmukh et al., ${ }^{3}$ Montmorillonite $\mathrm{K}-10$ clay $(0.50 \mathrm{~g})$, $\mathrm{Ac}_{2} \mathrm{O}$ (4.6 mL, $48 \mathrm{mmol}, 1.3$ equiv), 2-chloroacetaldehyde (3.1 mL, $45 \mathrm{mmol}, 1.2$ equiv), dimethyl malonate (33) $(4.3 \mathrm{~mL}, 4.4 \mathrm{mmol}, 1$ equiv) were stirred under nitrogen atmosphere in flamed dried flask at $70{ }^{\circ} \mathrm{C}$ for $3 \mathrm{~h} 30$. The reaction was cooled down to room temperature and saturated solution of $\mathrm{NaHCO}_{3}(20 \mathrm{~mL})$ was added, the mixture was extracted with ether $(3 \times 20$ $\mathrm{mL}$ ). The combined organic layers were dry over magnesium sulfate, filtrated and concentrated under reduced pressure. The crude residue was purified by column chromatography using a mixture of pentane and ethyl acetate (100\% pentane to $80: 20 \%$ pentane/ ethyl acetate) affording the pure product 35 (4.33 g, $22.5 \mathrm{mmol}, 61 \%$ yield) as a slightly yellow liquid.

RF $($ AcOEt/Pent $(1: 9)=0.43$.

${ }^{1} \mathrm{H}$ NMR $(400 \mathrm{MHz}$, Chloroform- $d) \delta 7.08(\mathrm{t}, J=7.3 \mathrm{~Hz}, 1 \mathrm{H}, \mathrm{C}=\mathrm{CH}), 4.34(\mathrm{~d}, J=7.3 \mathrm{~Hz}, 2 \mathrm{H}$, $\mathrm{CH}_{2}$ ), 3.88 (s, 3H, Me ester), 3.85 (s, 3H, Me ester).

${ }^{13} \mathrm{C}$ NMR (101 MHz, Chloroform-d) $\delta 164.3,163.6,144.2,129.1,52.8,52.7,39.2$.

IR 2951 (w), 1732 (s), $1439(\mathrm{~m}), 1282(\mathrm{~s}), 1227$ (s), $1058(w), 769(w)$.

HRMS (ESI) calcd for $\mathrm{C}_{7} \mathrm{H}_{9} \mathrm{ClNaO}_{4}{ }^{+}[\mathrm{M}+\mathrm{Na}]^{+} 215.0082$; found 215.0087 .

${ }^{3}$ A. R. A. S. Deshmukh, D. G. Panse, B. M. Bhawal, Synth. Commun. 1999, 29, 1801-1809. 
Dipentyl malonate (46).

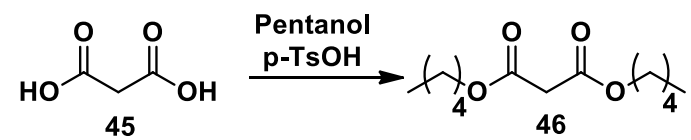

Following the procedure described by Tian et al. ${ }^{4}, 4$-methylbenzenesulfonic acid monohydrate ( $0.033 \mathrm{~g}, 0.19 \mathrm{mmol}, 0.01$ equiv), pentan-1-ol (4,6 mL, $42 \mathrm{mmol}, 2.2$ equiv), malonic acid (45) (2.00 g, $19.2 \mathrm{mmol}, 1$ equiv) were stirred at $85^{\circ} \mathrm{C}$ in a flame dried flask under nitrogen in benzene $(40 \mathrm{~mL})$ for $3 \mathrm{~h}$. The mixture was cooled down to room temperature and a saturated solution of $\mathrm{NaHCO}_{3}(20 \mathrm{~mL})$ was added, the mixture was extracted with ethyl acetate $(3 \times 20$ $\mathrm{mL})$. The combined organic layers were washed with saturated solution of $\mathrm{Na}_{2} \mathrm{CO}_{3}(20 \mathrm{~mL})$, dry over magnesium sulfate, filtrated and concentrated under reduced pressure. The crude product 46 (3.4 g, $14 \mathrm{mmol}, 73 \%$ yield) obtained as a yellow liquid after concentration under reduced pressure was clean enough to be used for the next step without further purification.

${ }^{1} \mathrm{H}$ NMR $(400 \mathrm{MHz}$, Chloroform- $d) \delta 4.17\left(\mathrm{t}, J=6.8 \mathrm{~Hz}, 4 \mathrm{H}\right.$, pentyl $\left.\mathrm{CH}_{2}\right), 3.40(\mathrm{~s}, 2 \mathrm{H}$, $\left.\mathrm{C}=\mathrm{OCH}_{2} \mathrm{C}=\mathrm{O}\right), 1.80-1.58\left(\mathrm{~m}, 4 \mathrm{H}\right.$, pentyl $\left.\mathrm{CH}_{2}\right), 1.36\left(\mathrm{~m}, 8 \mathrm{H}\right.$, pentyl $\left.\mathrm{CH}_{2}\right), 1.02-0.82(\mathrm{~m}, 6 \mathrm{H}$, pentyl $\mathrm{CH}_{3}$ ).

${ }^{13} \mathrm{C}$ NMR $\left(101 \mathrm{MHz}, \mathrm{CDCl}_{3}\right) \delta 166.7,65.7,41.7,28.2,27.9,22.3,14.0$.

NMR value correspond to literature. ${ }^{3}$

Dipentyl 2-(2-chloroethylidene)malonate (37).

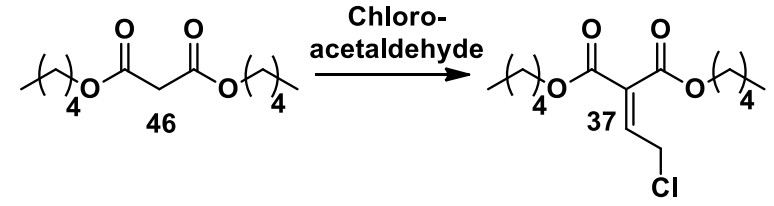

Following an adapted procedure from Deshmukh et al., ${ }^{3}$ Montmorillonite $\mathrm{K}-10$ clay $(80 \mathrm{mg})$, $\mathrm{Ac}_{2} \mathrm{O}$ (2.5 mL, $27 \mathrm{mmol}, 1.3$ equiv), 2-chloroacetaldehyde (1.7 mL, $25 \mathrm{mmol}, 1.3$ equiv), dipentyl malonate (46) $(5.0 \mathrm{~g}, 20 \mathrm{mmol}, 1$ equiv) were stirred under nitrogen atmosphere in a flamed dried flask at $70^{\circ} \mathrm{C}$ for 16 hours. The reaction was cooled down to room temperature and a saturated solution of $\mathrm{NaHCO}_{3}(20 \mathrm{~mL})$ was added, the mixture was extracted with ether (3x $20 \mathrm{~mL})$. The combined organic layers were dried over magnesium sulfate, filtrated and concentrated under reduced pressure. The crude oil was used without any purification. In general, the crude contain a ratio $7: 3$ product $\mathbf{3 7}$ to product $\mathbf{4 6}$.

\subsection{Pyrimidine cyclopropanes.}

1-Vinylthymine (10a).

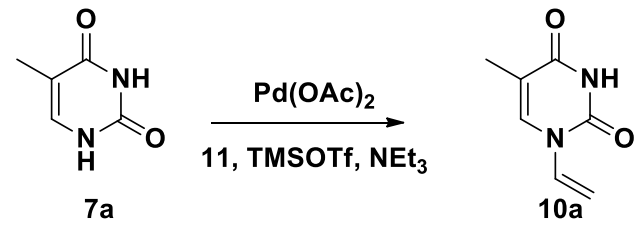

Thymine (7a) (0.50 g, $3.9 \mathrm{mmol}, 1.0 \mathrm{eq})$, diacetoxypalladium (0.036 g, $0.16 \mathrm{mmol}, 0.04 \mathrm{eq})$ were suspended in DMF $(10 \mathrm{~mL})$ in a flame-dried sealed microwave vial under nitrogen atmosphere. Vinyl acetate (11) $(0.82 \mathrm{~g}, 9.5 \mathrm{mmol}$, and $2.4 \mathrm{eq})$, triethylamine $(1.3 \mathrm{~mL}, 9.5 \mathrm{mmol}$, $2.4 \mathrm{eq})$ and TMSOTf $(1.7 \mathrm{~mL}, 9.5 \mathrm{mmol}$, and $2.4 \mathrm{eq})$ were added to the reaction mixture and stirred at $70{ }^{\circ} \mathrm{C}$ for 24 hours. The reaction mixture was cooled down to room temperature and the solvent was removed under reduced pressure. The crude mixture was purified by column

\footnotetext{
${ }^{4}$ Y. Tian, E. Akiyama, Y. Nagase, J. Mater. Chem. 2003, 13, 1253.
} 
chromatography, eluting with a mixture of ethyl acetate/ pentane (7:3). The pure 1-vinylthymine (10a) $(0.47 \mathrm{~g}, 3.1 \mathrm{mmol}, 79 \%$ yield) was obtained as white solid.

$\mathbf{R F}(\mathrm{Hex} / \mathrm{AcOEt}(1: 1))=0.5$.

Mp 208.0-209. $1^{\circ} \mathrm{C}$.

${ }^{1} \mathrm{H}$ NMR $(400 \mathrm{MHz}$, Chloroform- $d) \delta 9.17(\mathrm{~s}, 1 \mathrm{H}, \mathrm{NH}), 7.34(\mathrm{~s}, 1 \mathrm{H}$, thymine $\mathrm{C}=\mathrm{C}-\mathrm{H}), 7.21$ (dd, $1 \mathrm{H}, J=16.0,9.1 \mathrm{~Hz}$, -vinyl-CH), 5.07 (dd, $1 \mathrm{H}, J=16.0,2.1 \mathrm{~Hz}$, vinyl-CH$)_{2}, 4.91(\mathrm{dd}, 1 \mathrm{H}, J=$ 9.1, $2.1 \mathrm{~Hz}$, vinyl- $\left.\mathrm{CH}_{2}\right), 1.99\left(\mathrm{~s}, 3 \mathrm{H}\right.$, thymine $\left.-\mathrm{CH}_{3}\right)$.

${ }^{13} \mathrm{C}$ NMR (101 MHz, Chloroform-d) $\delta 163.6,149.3,134.5,129.6,112.1,100.5,12.6$.

IR 3173 (w), 3048 (w), 1698 (s), 1644 (s), 1459 (w), 1381 (w), 1344 (m), 1278 (m), 1129 (w).

HRMS (ESI) calcd for $\mathrm{C}_{7} \mathrm{H}_{9} \mathrm{~N}_{2} \mathrm{O}_{2}{ }^{+}[\mathrm{M}+\mathrm{H}]^{+}$153.0659; found 153.0653 .

Tert-butyl 5-methyl-2,6-dioxo-3-vinyl-2,3-dihydropyrimidine-1(6H)-carboxylate (12a).<smiles>CCn1cc(C)c(=O)[nH]c1=O</smiles>

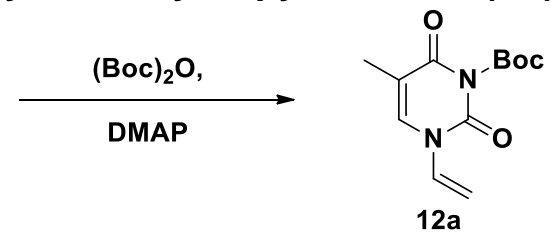

1-Vinylthymine 10a $(0.92 \mathrm{~g}, 6.1 \mathrm{mmol}, 1.0$ equiv), di-tert-butyl dicarbonate $(2.64 \mathrm{~g}, 12.1 \mathrm{mmol}$, 2.0 equiv) and dimethylaminopyridine $(1.48 \mathrm{~g}, 12.1 \mathrm{mmol}, 2.0$ equiv) were stirred in acetonitrile $(25.0 \mathrm{~mL})$ for $12 \mathrm{~h}$ in a flame-dried flask under nitrogen. Silica and triethylamine $(0.5 \mathrm{~mL})$ were added to the reaction and the solvent was removed under reduced pressure. The dry residue was purified by column chromatography using a mixture of hexane/ethyl acetate/1\% $\mathrm{NEt}_{3}$ (95:5 to $80: 20)$ as eluting solvent. The pure product $12 \mathrm{a}(1.15 \mathrm{~g}, 4.56 \mathrm{mmol}, 75 \%$ yield $)$ was obtained as a white solid.

$\mathbf{R F}(\mathrm{Hex} / \operatorname{AcOEt}(9: 1))=0.2$

Mp 109.9-111.2 ${ }^{\circ} \mathrm{C}$.

${ }^{1} \mathrm{H}$ NMR $(400 \mathrm{MHz}$, Chloroform- $d) \delta 7.31(\mathrm{~m}, 1 \mathrm{H}$, thymine $\mathrm{C}=\mathrm{C}-\mathrm{H}), 7.15(\mathrm{dd}, J=16.0,9.1 \mathrm{~Hz}$, $1 \mathrm{H}$, -vinyl-CH), 5.09 (dd, $J=16.0,2.2 \mathrm{~Hz}, 1 \mathrm{H}$, vinyl- $\mathrm{CH}_{2}$ ), 4.94 (dd, $J=9.1,2.2 \mathrm{~Hz}, 1 \mathrm{H}$, vinyl$\left.\mathrm{CH}_{2}\right), 1.99$ (s, $3 \mathrm{H}$, thymine- $\left.\mathrm{CH}_{3}\right), 1.60$ (s, $9 \mathrm{H}$, Boc).

${ }^{13} \mathrm{C}$ NMR $(400 \mathrm{MHz}$, Chloroform- $d) \delta 161.0,147.6,147.5,134.0,129.6,111.8,101.3,87.1$, $27.5,12.7$.

IR 2982 (w), 2937 (w), 1778 (s), 1721 (s), $1672($ s).

HRMS (ESI) calcd for $\mathrm{C}_{12} \mathrm{H}_{16} \mathrm{~N}_{2} \mathrm{NaO}_{4}{ }^{+}[\mathrm{M}+\mathrm{Na}]^{+}$275.1002; found 275.1008.

Dimethyl 2-(3-(tert-butoxycarbonyl)-5-methyl-2,4-dioxo-3,4-dihydropyrimidin-1(2H)yl)cyclopropane-1,1-dicarboxylate (13a).

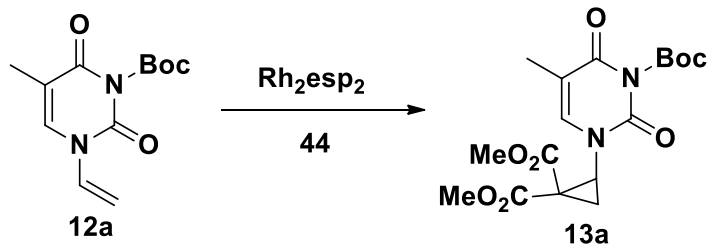


Diazomalonate 44 (1.80 g, $10.5 \mathrm{mmol}, 1.2$ equiv), $\mathrm{Rh}_{2}(\mathrm{esp})_{2}(0.013 \mathrm{~g}, 0.017 \mathrm{mmol}, 0.02 \mathrm{~mol} \%)$ and tert-butyl 5-methyl-2,6-dioxo-3-vinyl-2,3-dihydropyrimidine-1(6H)-carboxylate (12a) $(2.2 \mathrm{~g}$, $8.7 \mathrm{mmol}, 1.0$ equiv) were stirred at room temperature in $\mathrm{DCM}(18 \mathrm{~mL})$ in a flame-dried flask under nitrogen. After $40 \mathrm{~min}^{\mathrm{NEt}} \mathrm{Ne}_{3}(0.4 \mathrm{~mL})$ and silica were added and the solvent was removed under reduced pressure. The dried residue was purified by column chromatography using a mixture of pentane/ethyl acetate/1\% $\mathrm{NEt}_{3}(1: 1)$ as solvent gradient. The pure product $13 \mathbf{a}$ (3.30 $\mathrm{g}, 8.63 \mathrm{mmol}, 99 \%$ yield) was obtained as a slightly yellow foamy oil. The cyclopropane $13 \mathrm{a}$ was obtained in $64 \%$ yield over the three steps.

RF (hexane/AcOEt/1\% NEt $3(1: 1))=0.26$.

${ }^{1} \mathrm{H}$ NMR $(400 \mathrm{MHz}$, Chloroform- $d) \delta 6.94(\mathrm{~m}, 1 \mathrm{H}$, thymine $\mathrm{C}=\mathrm{CH}), 4.01(\mathrm{dd}, J=8.3,6.4 \mathrm{~Hz}$, $1 \mathrm{H}$, cyclopropane- $\mathrm{CH}), 3.79\left(\mathrm{~s}, 3 \mathrm{H}\right.$, ester- $\left.\mathrm{CH}_{3}\right), 3.71\left(\mathrm{~s}, 3 \mathrm{H}\right.$, ester- $\left.\mathrm{CH}_{3}\right), 2.27(\mathrm{t}, J=6.5 \mathrm{~Hz}, 1 \mathrm{H}$, cyclopropane- $\left.\mathrm{CH}_{2}\right), 1.91\left(\mathrm{~m}, 4 \mathrm{H}\right.$, thymine- $\mathrm{CH}_{3}$ and cyclopropane- $\left.\mathrm{CH}_{2}\right), 1.58(\mathrm{~s}, 9 \mathrm{H}, \mathrm{Boc})$.

${ }^{13} \mathrm{C}$ NMR (101 MHz Chloroform- $\left.d\right) \delta 167.6,166.2,161.2,149.1,147.4,138.2,110.5,86.8$, 53.3, 53.2, 42.9, 35.0, 27.5, 20.0, 12.5.

IR 3431 (w), 3364 (w), 2943 (m), 2866 (m), 2092 (w), 1705 (s), $1628(\mathrm{~m}), 1505(\mathrm{~m}), 1364(\mathrm{~m})$, 1167 (s).

HRMS (ESI) calcd for $\mathrm{C}_{17} \mathrm{H}_{22} \mathrm{~N}_{2} \mathrm{NaO}_{8}{ }^{+}[\mathrm{M}+\mathrm{Na}]^{+}$405.1268; found 405.1271.

Dibenzyl 2-(3-(tert-butoxycarbonyl)-5-methyl-2,4-dioxo-3,4-dihydropyrimidin-1(2H)yl)cyclopropane-1,1-dicarboxylate (47).

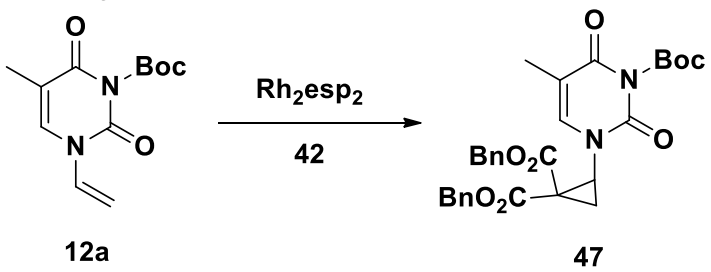

Tert-butyl 5-methyl-2,6-dioxo-3-vinyl-2,3-dihydropyrimidine-1(6H)-carboxylate (12a) (0.30 g, $1.2 \mathrm{mmol}, 1.0$ equiv) and $\mathrm{Rh}_{2}$ (esp) $)_{2}(1.8 \mathrm{mg}, 2.4 \mu \mathrm{mol}, 0.02 \mathrm{~mol} \%)$ were stirred in a flame-dried flask under nitrogen atmosphere with anhydrous DCM $(2.3 \mathrm{~mL})$ and diazomalonate $42(0.45$ $\mathrm{g}, 1.4 \mathrm{mmol}, 1.2$ equiv) was added at $0^{\circ} \mathrm{C}$. Then, the reaction mixture was allowed to warm up to room temperature and stirred for $14 \mathrm{~h}$. Silica and triethylamine $(0.5 \mathrm{~mL})$ were added and the solvent was removed under reduced pressure. The dried residue was purified by column chromatography using a mixture of pentane/ethyl acetate/1\% $\mathrm{NEt}_{3}(9: 1)$ as eluting solvent to afford dibenzyl 2-(3-(tert-butoxycarbonyl)-5-methyl-2,4-dioxo-3,4-dihydropyrimidin-1(2H)yl)cyclopropane-1,1-dicarboxylate (47) $(0.60 \mathrm{~g}, 1.1 \mathrm{mmol}, 93 \%$ yield) as a slightly yellow foam. The cyclopropane $\mathbf{4 7}$ was obtained in $60 \%$ yield over the three steps.

MP $76.9-82.1^{\circ} \mathrm{C}$.

$\mathbf{R F}($ pent/AcOEt $(7: 3))=0.29$.

${ }^{1}$ H NMR (400 MHz, Chloroform- $d$ ) $\delta 7.35-7.22(\mathrm{~m}, 10 \mathrm{H}, \mathrm{Ar}-\mathrm{H}), 6.83(\mathrm{q}, J=1.1 \mathrm{~Hz}, 1 \mathrm{H}$, thymine vinyl-CH), $5.27-5.10\left(\mathrm{~m}, 4 \mathrm{H}\right.$, benzylic- $\left.\mathrm{CH}_{2}\right), 4.05(\mathrm{dd}, J=8.3,6.5 \mathrm{~Hz}, 1 \mathrm{H}$, cyclopropane- $\mathrm{NCH}$ ), 2.31 (t, $J=6.6 \mathrm{~Hz}, 1 \mathrm{H}$, cyclopropane $\left.-\mathrm{CH}_{2}\right), 1.93(\mathrm{dd}, J=8.3,6.6 \mathrm{~Hz}, 1 \mathrm{H}$, cyclopropane $\left.-\mathrm{CH}_{2}\right), 1.78(\mathrm{~d}, \mathrm{~J}=1.3 \mathrm{~Hz}, 3 \mathrm{H}$, thymine methyl), 1.59 (s, 9H, Boc).

${ }^{13} \mathrm{C}$ NMR (101 MHz, Chloroform-d) $\delta 167.2,165.4,161.1,149.2,147.5,138.0,135.0,134.8$, 128.6, 128.6, 128.5, 128.4, 128.2, 110.5, 86.8, 68.3, 68.0, 43.1, 35.3, 27.5, 20.3, 12.4.

One carbone not resolved. 
IR 3066 (w), 2984 (w), 2932 (w), 1783 (s), 1725 (s), 1670 (s), 1433 (m), 1373 (m), 1316 (s), 1146 (s).

HRMS (ESI) calcd for $\mathrm{C}_{29} \mathrm{H}_{30} \mathrm{~N}_{2} \mathrm{NaO}_{8}{ }^{+}[\mathrm{M}+\mathrm{Na}]^{+} 557.1894$; found 557.1885.

1-Vinylpyrimidine-2,4(1H,3H)-dione (10b).<smiles>O=C1C=CNC1=O</smiles>

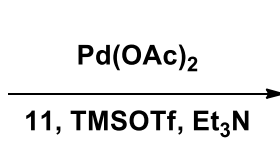

(1)

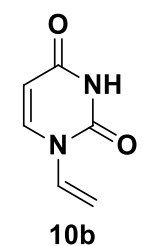

$10 \mathrm{~b}$

Palladium acetate $(0.036 \mathrm{~g}, 0.040 \mathrm{mmol}, 0.04$ equiv), vinyl acetate $(\mathbf{1 1})(0.87 \mathrm{~mL}, 10 \mathrm{mmol}$, 2.4 equiv), uracil (7b) ( $0.45 \mathrm{~g}, 4.0 \mathrm{mmol}, 1.0$ equiv), TMSOTf (1.7 mL, $9.5 \mathrm{mmol}, 2.4$ equiv) and triethylamine (1.4 mL, $9.5 \mathrm{mmol}, 2.4$ equiv) were stirred in DMF (11.5 mL) for 16 hours at $70{ }^{\circ} \mathrm{C}$ in a flame-dried sealed flask under nitrogen atmosphere. Then the reaction mixture was cooled down to room temperature and filtered on celite with AcOEt $(50 \mathrm{~mL})$. The crude mixture was concentrated under reduced pressure and purified by column chromatography, eluting with pentane and AcOEt mixture (2:8) to obtain the pure product $10 \mathrm{~b}(0.38 \mathrm{~g}, 2.7 \mathrm{mmol}, 69 \%$ yield) as a white solid.

$\mathbf{R F}($ AcOEt/pentane $(1: 1))=0.20$.

MP $175.2-176.7^{\circ} \mathrm{C}$.

${ }^{1} \mathrm{H}$ NMR (400 MHz, Acetone) $\delta 9.21(\mathrm{~s}, 1 \mathrm{H}$, uracil $\mathrm{N}-\mathrm{H}), 7.53(\mathrm{~d}, J=8.1 \mathrm{~Hz}, 1 \mathrm{H}$, uracil $\mathrm{CH})$, $7.23(\mathrm{dd}, J=16.0,9.0 \mathrm{~Hz}, 1 \mathrm{H}$, vinyl-CH), $5.86(\mathrm{dd}, J=8.1,1.3 \mathrm{~Hz}, 1 \mathrm{H}$, uracil $\mathrm{CH}), 5.13(\mathrm{dd}, J$ $=16.0,2.3 \mathrm{~Hz}, 1 \mathrm{H}$, vinyl- $\left.\mathrm{CH}_{2}\right), 5.00\left(\mathrm{dd}, J=9.0,2.3 \mathrm{~Hz}, 1 \mathrm{H}\right.$, vinyl- $\left.\mathrm{CH}_{2}\right)$.

${ }^{13} \mathrm{C}$ NMR (101 MHz, Acetone) $\delta 162.5,149.3,139.1,129.7,102.8,99.7$.

IR 3015 (w), 2823 (w), 1698 (s), 1640 (s), 1385 (s), 1278 (m), 1203 (m), 827 (s).

HRMS (ESI) calcd for $\mathrm{C}_{6} \mathrm{H}_{7} \mathrm{~N}_{2} \mathrm{O}_{2}{ }^{+}[\mathrm{M}+\mathrm{H}]^{+}$139.0502; found 139.0507 .

Tert-butyl 2,6-dioxo-3-vinyl-3,6-dihydropyrimidine-1(2H)-carboxylate (12b).

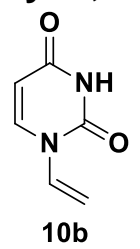<smiles>CCO[PbH2]C</smiles>

$10 \mathrm{~b}$<smiles>C=Cn1ccc(=O)n(C(=O)OC(C)(C)C)c1=O</smiles>

12b

1-Vinyluracil (10b) $(0.25 \mathrm{~g}, 1.8 \mathrm{mmol}, 1.0$ equiv), di-tert-butyl dicarbonate $(0.78 \mathrm{~g}, 3.6 \mathrm{mmol}$, 2.0 equiv) and dimethylaminopyridine $(0.44 \mathrm{~g}, 3.6 \mathrm{mmol}, 2.0$ equiv) were stirred in acetonitrile $(8.5 \mathrm{~mL})$ for $12 \mathrm{~h}$ in a flame-dried flask under nitrogen. Silica and triethylamine $(0.5 \mathrm{~mL})$ were added to the reaction and the solvent was removed under reduced pressure. The dry residue was purified by column chromatography eluting with pentane/AcOEt mixture $(2: 8)$ as solvent. The pure product $12 \mathrm{~b}(0.43 \mathrm{~g}, 1.8 \mathrm{mmol}$, quantitative yield) was obtained as a yellow oil.

$\mathbf{R F}($ AcOEt/pentane $(1: 1))=0.40$.

${ }^{1} \mathrm{H}$ NMR $(400 \mathrm{MHz}$, Chloroform- $d) \delta 7.51(\mathrm{~d}, J=8.2 \mathrm{~Hz}, 1 \mathrm{H}$, uracil $\mathrm{CH}), 7.15-7.02(\mathrm{~m}, 1 \mathrm{H}$, vinyl-CH), $5.80(\mathrm{~d}, J=8.2 \mathrm{~Hz}, 1 \mathrm{H}$, uracil $\mathrm{CH}), 5.14\left(\mathrm{dd}, J=16.0,2.4 \mathrm{~Hz}, 1 \mathrm{H}\right.$, vinyl- $\left.\mathrm{CH}_{2}\right), 4.96$ (dd, $J=9.0,2.5 \mathrm{~Hz}, 1 \mathrm{H}$, vinyl- $\mathrm{CH}_{2}$ ), 1.55 (s, 9H, Boc). 
${ }^{13} \mathrm{C}$ NMR (101 MHz, Chloroform- $\left.d\right) \delta 160.0,147.5,147.4,138.6,129.5,103.0,102.5,87.0$, 27.4.

IR 3104 (w), 2984 (w), 1783 (s), 1673 (s), 1440 (m), 1372 (s), 1280 (s), 1144 (s), 803 (m).

HRMS (ESI) calcd for $\mathrm{C}_{11} \mathrm{H}_{14} \mathrm{~N}_{2} \mathrm{NaO}_{4}{ }^{+}[\mathrm{M}+\mathrm{Na}]^{+} 261.0846$; found 261.0859.

Dimethyl 2-(3-(tert-butoxycarbonyl)-2,4-dioxo-3,4-dihydropyrimidin-1(2H)yl)cyclopropane-1,1-dicarboxylate (13b).

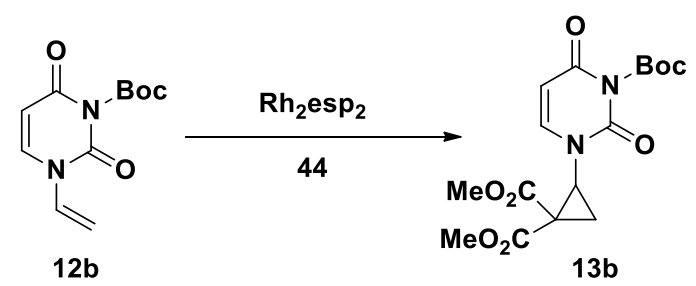

Diazomalonate 44 (0.28 g, $1.8 \mathrm{mmol}, 1.0$ equiv), $\mathrm{Rh}_{2}(\mathrm{esp})_{2}(2.7 \mathrm{mg}, 3.5 \mu \mathrm{mol}, 0.02 \mathrm{~mol} \%$ ) and product $12 \mathrm{~b}(0.42 \mathrm{~g}, 1.8 \mathrm{mmol}, 1.0$ equiv) were stirred at room temperature in $\mathrm{DCM}(18 \mathrm{~mL})$ in a flame-dried flask under nitrogen. After 2 hour. $\mathrm{NEt}_{3}(0.1 \mathrm{~mL})$ and silica were added and the solvent was removed under reduced pressure. The dried residue was purified by column chromatography using a mixture of pentane/AcOEt (3:7) as eluting solvent. The pure cyclopropane $13 \mathrm{~b}(0.56 \mathrm{~g}, 1.5 \mathrm{mmol}, 87 \%$ yield $)$ was obtained as a colorless foam. The cyclopropane $13 \mathrm{~b}$ was obtained in $60 \%$ yield over the three steps.

$\mathbf{R F}($ AcOEt/pentane $(1: 1))=0.20$.

MP $45.0-46.2^{\circ} \mathrm{C}$.

${ }^{1} \mathrm{H}$ NMR $(400 \mathrm{MHz}$, Chloroform- $d) \delta 7.08(\mathrm{~d}, J=8.1 \mathrm{~Hz}, 1 \mathrm{H}$, uracil $\mathrm{CH}), 5.64(\mathrm{~d}, J=8.2 \mathrm{~Hz}$, $1 \mathrm{H}$, uracil $\mathrm{CH}$ ), 3.98 (dd, $J=8.2,6.5 \mathrm{~Hz}, 1 \mathrm{H}$, cyclopropane- $\mathrm{CH}), 3.73\left(\mathrm{~s}, 3 \mathrm{H}\right.$, ester $\left.\mathrm{CH}_{3}\right), 3.65$ $\left(\mathrm{s}, 3 \mathrm{H}\right.$, ester $\left.\mathrm{CH}_{3}\right), 2.19\left(\mathrm{t}, J=6.6 \mathrm{~Hz}, 1 \mathrm{H}\right.$, cyclopropane- $\left.\mathrm{CH}_{2}\right), 1.88(\mathrm{dd}, J=8.5,6.8 \mathrm{~Hz}, 1 \mathrm{H}$, cyclopropane- $\mathrm{CH}_{2}$ ), 1.51 (s, 9H, Boc).

${ }^{13}$ C NMR (101 MHz, Chloroform- $\left.d\right) \delta 167.5,166.1,160.2,149.1,147.2,142.4,101.9,87.0$, 53.3, 53.2, 43.0, 34.9, 27.4, 19.8 .

IR 1780 (w), $1723(\mathrm{~m}), 1676$ (s), 1435 (w), $1312(\mathrm{~m}), 1145$ (s), 733 (s).

HRMS (ESI) calcd for $\mathrm{C}_{16} \mathrm{H}_{20} \mathrm{~N}_{2} \mathrm{NaO}_{8}{ }^{+}[\mathrm{M}+\mathrm{Na}]^{+} 391.1112$; found 391.1106.

Dimethyl 2-(4-(2-((tert-butoxycarbonyl)oxy)-1,3-dimethoxy-1,3-dioxopropan-2-yl)-2oxopyrimidin-1(2H)-yl)cyclopropane-1,1-dicarboxylate (14).

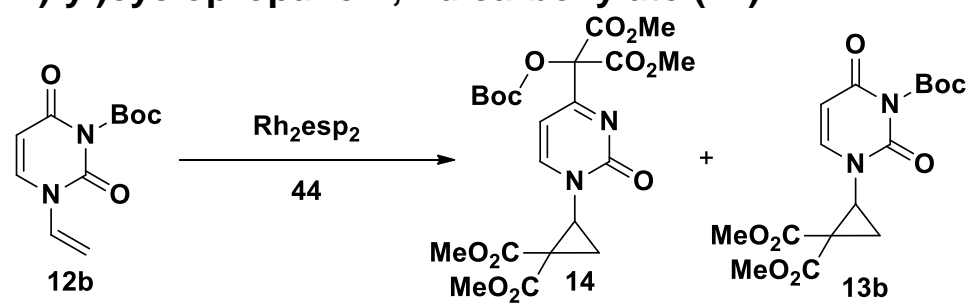

Dimethyl 2-diazomalonate (44) (0.51 g, $3.2 \mathrm{mmol}, 1.2$ equiv), $\mathrm{Rh}_{2}(\mathrm{esp})_{2}$ (4 mg, $5 \mu \mathrm{mol}, 0.02$ mol\%) and product $12 \mathrm{~b}(0.64 \mathrm{~g}, 2.7 \mathrm{mmol}, 1.0$ equiv) were stirred at room temperature in DCM $(18 \mathrm{~mL})$ in a flame-dried flask under nitrogen. After 2 hour. $\mathrm{NEt}_{3}(0.1 \mathrm{~mL})$ and silica were added 
and the solvent was removed under reduced pressure. The dried residue was purified by column chromatography using a mixture of pentane/AcOEt (3:7) as eluting solvent. The pure product $14\left(0.51 \mathrm{~g}, 1.0 \mathrm{mmol}, 64 \%\right.$ yield $\left.^{5}\right)$ was obtained as white solid and the pure product $13 \mathrm{~b}$ ( $0.33 \mathrm{~g}, 0.90 \mathrm{mmol}, 28 \%$ yield) as colorless foam.

$\mathbf{R F}($ AcOEt/pentane $(1: 1))=0.18$.

MP $159.1-160.3^{\circ} \mathrm{C}$.

${ }^{1} \mathrm{H}$ NMR $(400 \mathrm{MHz}$, Chloroform- $d) \delta 7.47(\mathrm{~d}, J=7.4 \mathrm{~Hz}, 1 \mathrm{H}$, uracil $\mathrm{CH}), 6.12(\mathrm{~d}, J=7.3 \mathrm{~Hz}$, $1 \mathrm{H}$, uracil $\mathrm{CH}$ ), 4.22 (dd, $J=8.2,6.7 \mathrm{~Hz}, 1 \mathrm{H}$, cyclopropane- $\mathrm{CH}), 3.88\left(\mathrm{~m}, 6 \mathrm{H}\right.$, ester $\left.\mathrm{CH}_{3}\right), 3.83$ (s, $3 \mathrm{H}$, ester $\left.\mathrm{CH}_{3}\right), 3.66\left(\mathrm{~s}, 3 \mathrm{H}\right.$, ester $\left.\mathrm{CH}_{3}\right), 2.31\left(\mathrm{t}, J=6.7 \mathrm{~Hz}, 1 \mathrm{H}\right.$, cyclopropane- $\left.\mathrm{CH}_{2}\right), 2.00$ (dd, $J=8.2,6.6 \mathrm{~Hz}, 1 \mathrm{H}$, cyclopropane- $\mathrm{CH}_{2}$ ), 1.52 (s, 9H, Boc).

${ }^{13} \mathrm{C}$ NMR $\left(101 \mathrm{MHz}, \mathrm{CDCl}_{3}\right) \delta 169.0,167.8,166.0,163.0,160.6,154.4,146.6,94.9,85.0,53.8$, 53.1, 53.1, 44.4, 34.9, 27.6, 19.4. Two carbons are not resolved.

IR 3101 (w), 2956 (w), 1737 (s), 1678 (s), 1539 (s), 1455 (m), 1330 (s), 1119 (s), 798 (m).

HRMS (ESI) calcd for $\mathrm{C}_{21} \mathrm{H}_{27} \mathrm{~N}_{2} \mathrm{O}_{12}{ }^{+}[\mathrm{M}+\mathrm{H}]^{+}$499.1559; found 499.1554.

\section{5-Fluoro-1-vinylpyrimidine-2,4(1H,3H)-dione (10c).}

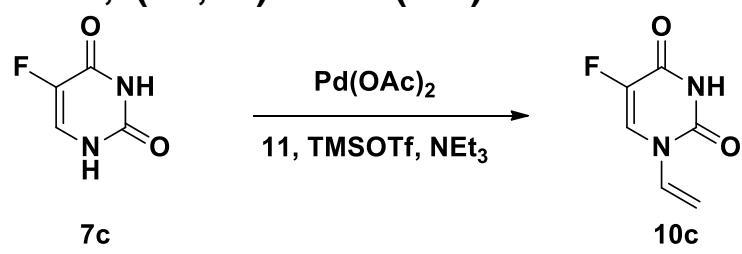

Palladium acetate $(0.013 \mathrm{~g}, 0.056 \mathrm{mmol}, 0.04$ equiv), vinyl acetate (11) $(1.7 \mathrm{~mL}, 3.4 \mathrm{mmol}, 2.4$ equiv), fluoro-uracil $7 \mathrm{c}(0.18 \mathrm{~g}, 1.4 \mathrm{mmol}, 1.0$ equiv), TMSOTf (0.60 mL, $3.4 \mathrm{mmol}, 2.4$ equiv) and triethylamine $(0.47 \mathrm{~mL}, 3.4 \mathrm{mmol}, 2.4$ equiv) were stirred in DMF (4 mL) for 16 hours at $70{ }^{\circ} \mathrm{C}$ in a flame-dried sealed flask under nitrogen atmosphere. Then the reaction mixture was cooled down to room temperature and filtered on celite with AcOEt $(50 \mathrm{~mL})$. The crude mixture was concentrated under reduced pressure and purified by column chromatography, eluting with pentane and AcOEt mixture (1:1) to obtain the pure product $10 \mathrm{c}(0.12 \mathrm{~g}, 0.58 \mathrm{mmol}, 57 \%$ yield) as a white solid.

$\mathbf{R F}($ AcOEt/pentane $(6: 4))=0.51$

MP $135.1-137.0^{\circ} \mathrm{C}$.

${ }^{1} \mathrm{H}$ NMR $(400 \mathrm{MHz}$, Chloroform- $d) \delta 7.52$ (d, $J=5.8 \mathrm{~Hz}, 1 \mathrm{H}$, F-uracil vinyl-CH ), 7.12 (ddd, $J=$ $15.9,9.1,1.8 \mathrm{~Hz}, 1 \mathrm{H}$, vinyl-CH), $5.11-4.79\left(\mathrm{~m}, 2 \mathrm{H}\right.$, vinyl- $\left.\mathrm{CH}_{2}\right)$.

${ }^{13} \mathrm{C}$ NMR (101 MHz, Chloroform- $d$ ) $\delta 156.1$ (d, $\left.J=27.3 \mathrm{~Hz}\right), 147.3,141.1$ (d, $J=241.8 \mathrm{~Hz}$ ), 129.3, 123.1 (d, $J=34.2 \mathrm{~Hz}), 101.3$.

IR 3031 (w), 2844 (w), 1661 (s), 1377 (s), 1268 (s), 1122 (s), 970 (m), 913 (m).

HRMS (ESI) calcd for $\mathrm{C}_{6} \mathrm{FH}_{6} \mathrm{~N}_{2} \mathrm{O}_{2}{ }^{+}[\mathrm{M}+\mathrm{H}]^{+}$157.0408; found 157.0414.

\section{3-Benzoyl-5-fluoro-1-vinylpyrimidine-2,4(1H,3H)-dione (12c).}

\footnotetext{
${ }^{5}$ Yield calculated from diazomalonate. If calculated with uracil as reference; 13b: 33\%, 14: $38 \%$.
} 


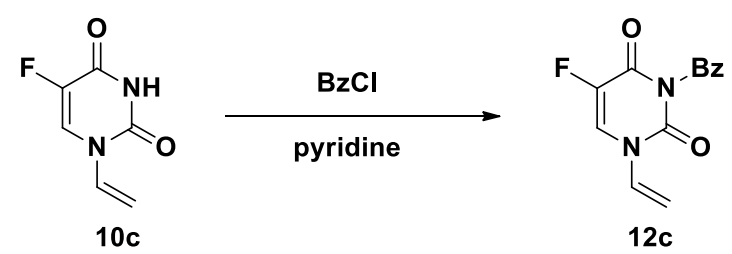

In a flame-dried flask under nitrogen, 1-vinyl-fluorouracil (10c) $(0.12 \mathrm{~g}, 0.79 \mathrm{mmol}, 1.0$ equiv) was stirred with pyridine $(2 \mathrm{~mL})$, and added dropwise over $10 \mathrm{~min}$ to a solution of benzoyl chloride $(0.34 \mathrm{~g}, 2.4 \mathrm{mmol}, 3$ equiv) in pyridine $(0.7 \mathrm{~mL})$ and stirred for $2 \mathrm{~h}$ at room temperature. Then the crude mixture was partitioned between water $(10 \mathrm{~mL})$ and AcOEt $(10 \mathrm{~mL})$. The aqueous layer was extracted three times with AcOEt $(10 \mathrm{~mL})$ and the organic layers were dried over anhydrous $\mathrm{MgSO}_{4}$. The solvent was removed under reduced pressure and the dry residue was purified by column chromatography using a mixture of pentane/AcOEt (7:3 to 1:1) as eluting solvent. The pure product $12 \mathrm{c}(0.15 \mathrm{~g}, 0.58 \mathrm{mmol}, 73 \%$ yield $)$ was obtained as a white solid.

$\mathbf{R F}($ AcOEt/pentane $(1: 1))=0.54$

MP $128.6-129.3^{\circ} \mathrm{C}$.

${ }^{1} \mathbf{H}$ NMR $(400 \mathrm{MHz}$, Chloroform- $d) \delta 7.95(\mathrm{dd}, J=8.3,1.4 \mathrm{~Hz}, 2 \mathrm{H}, \mathrm{Ar}-\mathrm{H}), 7.75-7.64(\mathrm{~m}, 2 \mathrm{H}$, Ar-H and F-uracil vinyl-CH), $7.52(\mathrm{t}, J=7.9 \mathrm{~Hz}, 2 \mathrm{H}, \mathrm{Ar}-\mathrm{H}$ ), 7.12 (ddd, $J=15.9,9.0,1.8 \mathrm{~Hz}, 1 \mathrm{H}$, vinyl-CH), 5.13 (dd, $J=15.8,2.7 \mathrm{~Hz}, 1 \mathrm{H}$, vinyl- $\mathrm{CH}_{2}$ ), 4.99 (dd, $J=9.0,2.8 \mathrm{~Hz}, 1 \mathrm{H}$, vinyl-CH 2 ).

${ }^{13} \mathrm{C}$ NMR (101 MHz, Chloroform- $d$ ) $\delta 166.8,155.8(\mathrm{~d}, J=27.5 \mathrm{~Hz}), 146.8,140.6(\mathrm{~d}, J=242.6$ $\mathrm{Hz}), 135.7,130.7,130.6,129.4,129.0,123.6$ (d, $J=34.2 \mathrm{~Hz}), 102.2$.

IR $1756(\mathrm{~m}), 1665$ (s), 1448 (w), 1368 (s), $1276(\mathrm{~m}), 1229$ (m), 909 (m).

HRMS (ESI) calcd for $\mathrm{C}_{13} \mathrm{H}_{9} \mathrm{FN}_{2} \mathrm{NaO}_{3}{ }^{+}[\mathrm{M}+\mathrm{Na}]^{+} 283.0489$; found 283.0485.

Dimethyl 2-(3-benzoyl-5-fluoro-2,4-dioxo-3,4-dihydropyrimidin-1(2H)-yl)cyclopropane1,1-dicarboxylate (13c).

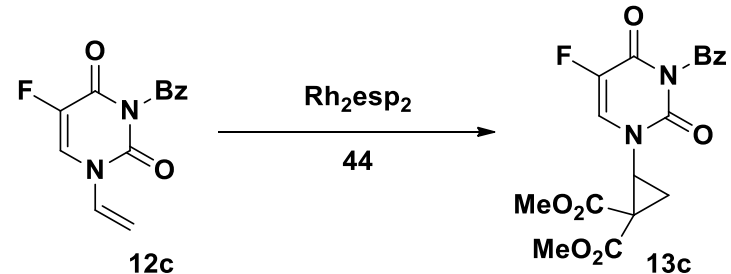

Diazomalonate 44 (0.21 g, $1.3 \mathrm{mmol}, 1.2$ equiv), $\mathrm{Rh}_{2}(\mathrm{esp})_{2}(0.002 \mathrm{~g}, 0.002 \mathrm{mmol}, 0.02 \mathrm{~mol} \%)$ and product $12 \mathrm{c}(0.29 \mathrm{~g}, 0.11 \mathrm{mmol}, 1.0$ equiv) were stirred at room temperature in DCM (11 $\mathrm{mL})$ in a flame-dried flask under nitrogen. After 1 hour, $\mathrm{NEt}_{3}(0.1 \mathrm{~mL})$ and silica were added and the solvent was removed under reduced pressure. The dried residue was purified by column chromatography using a mixture of pentane/AcOEt (3:7) as eluting solvent. The pure cyclopropane $13 \mathrm{c}(0.40 \mathrm{~g}, 1.0 \mathrm{mmol}, 93 \%$ yield) was obtained as a white foamy solid. The cyclopropane was obtained in $39 \%$ yield over the three steps.

$\mathbf{R F}($ AcOEt/pentane $(3: 7))=0.33$.

MP $62.7-64.4^{\circ} \mathrm{C}$.

${ }^{1}$ H NMR $(400 \mathrm{MHz}$, Chloroform- $d) \delta 7.85-7.78(\mathrm{~m}, 2 \mathrm{H}, \mathrm{Ar}-\mathrm{H}), 7.62-7.55(\mathrm{~m}, 1 \mathrm{H}, \mathrm{Ar}-\mathrm{H}), 7.45$ $-7.39(\mathrm{~m}, 2 \mathrm{H}, \mathrm{Ar}-\mathrm{H}), 7.32(\mathrm{~d}, J=5.6 \mathrm{~Hz}, 1 \mathrm{H}, \mathrm{F}$-uracil vinyl-CH), $3.89(\mathrm{dd}, J=8.0,6.3 \mathrm{~Hz}, 1 \mathrm{H}$, cyclopropane- $\mathrm{CH}), 3.68\left(\mathrm{~s}, 3 \mathrm{H}\right.$, ester $\left.\mathrm{CH}_{3}\right), 3.58\left(\mathrm{~s}, 3 \mathrm{H}\right.$, ester $\left.\mathrm{CH}_{3}\right), 2.09(\mathrm{t}, J=6.5 \mathrm{~Hz}, 1 \mathrm{H}$, cyclopropane- $\mathrm{CH}$ ), 1.91 (dd, $J=8.1,6.6 \mathrm{~Hz}, 1 \mathrm{H}$, cyclopropane- $\mathrm{CH}$ ). 
${ }^{13} \mathrm{C}$ NMR $(101 \mathrm{MHz}$, Chloroform- $d) \delta 167.1,166.7,156.0(\mathrm{~d}, J=27.2 \mathrm{~Hz}), 148.7,139.7$ (d, $J=$ 240.3 Hz), 135.6, 130.8, 130.7, 129.2, 128.0 (d, J = 33.7 Hz), 53.3, 53.3, 42.9, 34.8, 20.3.

One $\mathrm{C}=\mathrm{O}$ of methyl-ester is not resolved.

IR 3084 (w), 2953 (w), 1755 (m), 1717 (s), 1440 (m), 1296 (s), 1227 (s), 911 (m), 729 (s).

HRMS (ESI) calcd for $\mathrm{C}_{18} \mathrm{H}_{15} \mathrm{FN}_{2} \mathrm{NaO}_{7}^{+}[\mathrm{M}+\mathrm{Na}]^{+}$413.0755; found 413.0763.

The crystal structure has been deposited at the Cambridge Crystallographic Data Centre and allocated the deposition number: CCDC 99618.

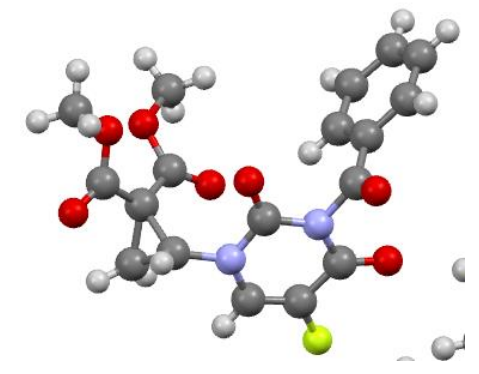

\subsection{Pyrimidine cyclobutane.}

Dimethyl 2-(5-methyl-2,4-dioxo-3,4-dihydropyrimidin-1(2H)-yl)cyclobutane-1,1dicarboxylate (16a).<smiles>C=Cn1cc(C)c(=O)[nH]c1=O</smiles>

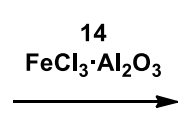

$10 \mathrm{a}$<smiles>COC(=O)C1(C(C)(C)C)CCC1n1cc(C)c(=O)[nH]c1=O</smiles>

16a $\mathrm{CO}_{2} \mathrm{Me}$

Following an adapted described procedure of De Nanteuil et al., ${ }^{1 \mathrm{C}} 5$-methyl-1-vinylpyrimidine2,4(1H,3H)-dione (10a) ( $0.43 \mathrm{~g}, 2.9 \mathrm{mmol}, 1.0$ equiv) and iron trichloride supported on alumina $(1.0 \mathrm{mmol} / \mathrm{g}, 0.57 \mathrm{~g}, 0.57 \mathrm{mmol}, 0.2$ equiv) were added in a flame dried microwave vial under nitrogen atmosphere. Then, dry dichloromethane $(7 \mathrm{~mL})$ and a solution of crude freshly prepared dimethyl 2-methylenemalonate (14) $(0.82 \mathrm{~g}, 5.7 \mathrm{mmol}, 2.0$ equiv) in dichloromethane $(3 \mathrm{~mL})$ were added into the vial and the reaction mixture was stirred at room temperature for 14 hours. The reaction mixture was filtrated over a pad of alumina eluting with ethyl acetate $(25 \mathrm{~mL})$ and concentrated under reduced pressure. The crude mixture was purified via column chromatography, eluting with a mixture of ethyl acetate/ pentane (6:4 to 9:1). The pure product $16 \mathrm{a}(0.36 \mathrm{~g}, 1.2 \mathrm{mmol}, 43 \%$ yield $)$ was obtained as a white solid.

RF $($ Pent/AcOEt $(2: 8))=0.21$

Mp: $180.4-182.1^{\circ} \mathrm{C}$.

${ }^{1} \mathrm{H}$ NMR $(400 \mathrm{MHz}$, Chloroform- $d) \delta 7.99(\mathrm{~s}, 1 \mathrm{H}, \mathrm{NH}), 7.05(\mathrm{~d}, \mathrm{~J}=1.3 \mathrm{~Hz}, 1 \mathrm{H}$, thymine C=C$\mathrm{H}), 5.33\left(\mathrm{t}, J=9.2 \mathrm{~Hz}, 1 \mathrm{H}\right.$, cyclobutane- $\mathrm{CH}$ ), 3.77 (s, $3 \mathrm{H}$, ester $\left.\mathrm{CH}_{3}\right), 3.69\left(\mathrm{~s}, 3 \mathrm{H}\right.$, ester $\left.\mathrm{CH}_{3}\right)$, $2.79-2.67\left(\mathrm{~m}, 2 \mathrm{H}\right.$, cyclobutane- $\left.\mathrm{CH}_{2}\right), 2.35\left(\mathrm{q}, \mathrm{J}=8.5 \mathrm{~Hz}, 1 \mathrm{H}\right.$, cyclobutane- $\left.\mathrm{CH}_{2}\right), 2.27-2.16$ (m, $1 \mathrm{H}$, cyclobutane- $\left.\mathrm{CH}_{2}\right), 1.93\left(\mathrm{~d}, J=1.2 \mathrm{~Hz}, 3 \mathrm{H}\right.$, thymine- $\left.\mathrm{CH}_{3}\right)$. 
${ }^{13}$ C NMR (101 MHz, Chloroform- $\left.d\right) \delta 170.0,168.8,163.9,151.0,137.7,110.3,59.1,55.0$, 53.2, 53.1, 23.7, 22.8, 12.5 .

IR: 3190 (w), 3034 (w), 2958 (w), $2361(\mathrm{~m}), 1733$ (s), 1690 (s), 1439 (w), 1278 (m), $1113(w)$, $915(w), 735(w)$.

HRMS (ESI) calcd for $\mathrm{C}_{13} \mathrm{H}_{16} \mathrm{~N}_{2} \mathrm{NaO}_{6}{ }^{+}[\mathrm{M}+\mathrm{Na}]^{+} 319.0901$, found 319.0904 .

Dimethyl 2-(3-(tert-butoxycarbonyl)-5-methyl-2,4-dioxo-3,4-dihydropyrimidin-1(2H)yl)cyclobutane-1,1-dicarboxylate (17).
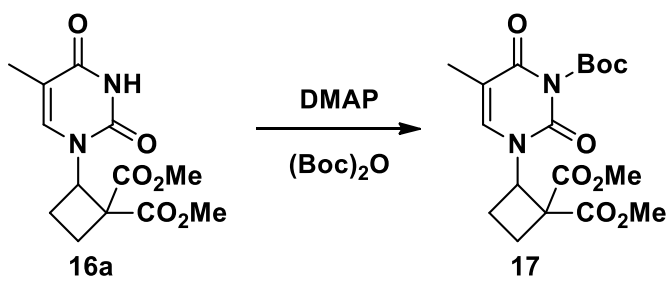

Cyclobutane $16 \mathrm{a}(0.20 \mathrm{~g}, 0.68 \mathrm{mmol}, 1.0$ equiv) and dimethyl-4-aminopyridine $(0.12 \mathrm{~g}, 1.0$ mmol, 1.5 equiv) were dissolved in acetonitrile $(3.0 \mathrm{~mL})$ in a flame dried flask under nitrogen atmosphere. A solution of di-tert-butyl dicarbonate $(0.29 \mathrm{~g}, 1.4 \mathrm{mmol}, 2.0$ equiv) in acetonitrile $(0.5 \mathrm{~mL})$ was added to the reaction mixture and it was stirred at room temperature for 14 hours. The solvent was evaporated under reduced pressure and the crude product was purified by column chromatography, eluting with a mixture of pentane/ ethyl acetate (8:2 to 6:4). The pure product 17 (0.22 g, $0.55 \mathrm{mmol}, 81 \%$ yield) was obtained as slightly yellow oil.

RF $($ Pent/AcOEt $(4: 6))=0.28$.

${ }^{1} \mathrm{H}$ NMR $(400 \mathrm{MHz}$, Chloroform- $d) \delta 7.05(\mathrm{~d}, J=1.3 \mathrm{~Hz}, 1 \mathrm{H}$, thymine $\mathrm{C}=\mathrm{C}-\mathrm{H}), 5.25-5.18(\mathrm{~m}$, $1 \mathrm{H}$, cyclobutane- $\mathrm{CH}), 3.76\left(\mathrm{~s}, 3 \mathrm{H}\right.$, ester $\left.\mathrm{CH}_{3}\right), 3.69\left(\mathrm{~s}, 3 \mathrm{H}\right.$, ester $\left.\mathrm{CH}_{3}\right), 2.98-2.83(\mathrm{~m}, 1 \mathrm{H}$, cyclobutane- $\left.\mathrm{CH}_{2}\right), 2.83-2.72\left(\mathrm{~m}, 1 \mathrm{H}\right.$, cyclobutane- $\left.\mathrm{CH}_{2}\right), 2.38-2.28(\mathrm{~m}, 1 \mathrm{H}$, cyclobutane$\left.\mathrm{CH}_{2}\right), 2.19-2.10\left(\mathrm{~m}, 1 \mathrm{H}\right.$, cyclobutane- $\left.\mathrm{CH}_{2}\right), 1.93\left(\mathrm{~d}, J=1.3 \mathrm{~Hz}, 3 \mathrm{H}\right.$, thymine $\left.-\mathrm{CH}_{3}\right), 1.59(\mathrm{~s}$, $9 \mathrm{H}, \mathrm{Boc})$.

${ }^{13} \mathrm{C}$ NMR (101 MHz, Chloroform- $\left.d\right) \delta 170.2,168.6,161.4,149.2,147.9,138.1,110.1,86.8$, $59.2,56.3,53.3,53.3,27.6,23.7,23.0,12.6$.

NMR values correspond to the literature. ${ }^{1 \mathrm{c}}$

Dimethyl 2-(3-benzoyl-5-methyl-2,4-dioxo-3,4-dihydropyrimidin-1(2H)-yl)cyclobutane1,1-dicarboxylate (18a).
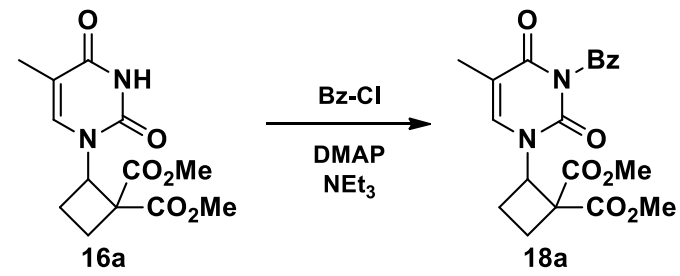
In a flame dried microwave vial under nitrogen atmosphere, cyclobutane $16 \mathrm{a}(0.32 \mathrm{~g}, 1.1 \mathrm{mmol}$, 1.0 equiv) and dimethyl-4-aminopyridin $(0.13 \mathrm{~g}, 1.1 \mathrm{mmol}, 1.0$ equiv) were dissolved in dry dichloromethane $(10 \mathrm{~mL})$. Then, triethylamine $(0.60 \mathrm{~mL}, 4.3 \mathrm{mmol}, 4.0$ equiv) was added and the solution was cooled to $0^{\circ} \mathrm{C}$. Benzoyl chloride $(0.38 \mathrm{~mL}, 3.2 \mathrm{mmol}, 3.0$ equiv) was added dropwise and the reaction mixture was stirred at room temperature for 14 hours. A saturated solution of $\mathrm{NaHCO}_{3}(8 \mathrm{~mL})$ was added to the reaction mixture and the layers were separated. The aqueous layer was extracted with dichloromethane $(2 \times 20 \mathrm{~mL})$. The combined organic layers were washed with $1 \mathrm{M} \mathrm{HCl}(2 \times 40 \mathrm{~mL})$ and with water $(30 \mathrm{~mL})$, dried over $\mathrm{MgSO}_{4}$ and concentrated under reduce pressure. The crude product was purified by column chromatography, eluting with a mixture of pentane/ ethyl acetate (4:6 to $2: 8)$. The pure product $18 \mathrm{a}(0.37 \mathrm{~g}, 0.93 \mathrm{mmol}, 86 \%$ yield) was obtained as colorless foam.

RF $($ Pent/AcOEt $(4: 6))=0.32$.

Mp: $153.8-155.4^{\circ} \mathrm{C}$.

${ }^{1} \mathrm{H}$ NMR (400 MHz, Chloroform- $\left.d\right) \delta 7.96-7.90(\mathrm{~m}, 2 \mathrm{H}, \mathrm{Ar}-\mathrm{H}), 7.65-7.59(\mathrm{~m}, 1 \mathrm{H}, \mathrm{Ar}-\mathrm{H}), 7.50$ - $7.44(\mathrm{~m}, 2 \mathrm{H}, \operatorname{Ar}-\mathrm{H}), 7.16(\mathrm{~d}, J=1.2 \mathrm{~Hz}, 1 \mathrm{H}$, thymine $\mathrm{C}=\mathrm{C}-\mathrm{H}), 5.17(\mathrm{t}, J=9.5 \mathrm{~Hz}, 1 \mathrm{H}$, cyclobutane- $\mathrm{CH}), 3.69\left(\mathrm{~s}, 3 \mathrm{H}\right.$, ester $\left.\mathrm{CH}_{3}\right), 3.66\left(\mathrm{~s}, 3 \mathrm{H}\right.$, ester $\left.\mathrm{CH}_{3}\right), 2.94-2.82(\mathrm{~m}, 1 \mathrm{H}$, cyclobutane- $\mathrm{CH}_{2}$ ), 2.74 (td, $J=11.0,3.1 \mathrm{~Hz}, 1 \mathrm{H}$, cyclobutane- $\mathrm{CH}_{2}$ ), 2.35 (dtd, $J=11.6,8.8$, $3.1 \mathrm{~Hz}, 1 \mathrm{H}$, cyclobutane- $\left.\mathrm{CH}_{2}\right), 2.18\left(\mathrm{dt}, J=11.7,8.9 \mathrm{~Hz}, 1 \mathrm{H}\right.$, cyclobutane- $\left.\mathrm{CH}_{2}\right), 1.95(\mathrm{~d}, J=$ $1.2 \mathrm{~Hz}, 3 \mathrm{H}$, thymine- $\mathrm{CH}_{3}$ ).

${ }^{13} \mathrm{C}$ NMR (101 MHz, Chloroform- $d$ ) $\delta$ 170.2, 168.9, 168.7, 162.8, 150.1, 138.7, 135.0, 131.6, $130.7,129.1,110.4,58.8,56.9,53.3,53.2,23.9,22.7,12.6$.

IR: 3006 (w), 2959 (w), 2358 (w), 2257 (w), 1737 (s), 1702 (m), 1655 (s), 1438 (m), 1266 (s), $1107(\mathrm{~m}), 984(\mathrm{w}), 914(\mathrm{w}), 733(\mathrm{~s})$.

HRMS (ESI) calcd for $\mathrm{C}_{20} \mathrm{H}_{20} \mathrm{~N}_{2} \mathrm{NaO}_{7}^{+}[\mathrm{M}+\mathrm{Na}]^{+}$423.1163; found 423.1168.

\section{Dimethyl 2-(5-fluoro-2,4-dioxo-3,4-dihydropyrimidin-1(2H)-yl)cyclobutane-1,1- dicarboxylate (16b).}<smiles>C=Cn1cc(F)c(=O)[nH]c1=O</smiles>
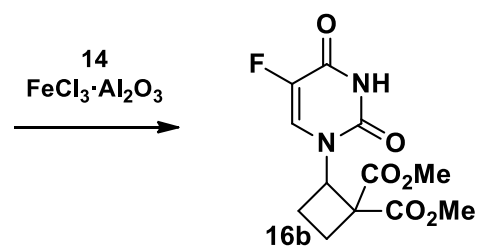

5-Fluoro-1-vinylpyrimidine-2,4(1H,3H)-dione (10b) $(0.60 \mathrm{~g}, 3.8 \mathrm{mmol}, 1.0 \mathrm{~g}$ equiv) and iron trichloride supported on alumina $(1.0 \mathrm{mmol} / \mathrm{g}, 0.77 \mathrm{~g}, 0.77 \mathrm{mmol}, 0.2$ equiv) were added in a flame dried microwave vial under nitrogen atmosphere. Then, dry dichloromethane $(8 \mathrm{~mL})$ and a solution of crude freshly prepared dimethyl 2-methylenemalonate (14) $(2.2 \mathrm{~g}, 15 \mathrm{mmol}, 4.0$ equiv) in dichloromethane $(2 \mathrm{~mL})$ were added into the vial and the reaction mixture was stirred at room temperature for 14 hours. The reaction mixture was filtrated over a pad of alumina eluting with ethyl acetate $(50 \mathrm{~mL})$ and concentrated under reduced pressure. The crude mixture was purified via column chromatography, eluting with a mixture of ethyl acetate/ pentane (5:5 to $2: 8)$. The pure product $16 \mathrm{~b}(0.78 \mathrm{~g}, 2.6 \mathrm{mmol}, 67 \%$ yield) was obtained as a 
colorless oil.

RF $($ Pent/AcOEt $(4: 6))=0.28$.

${ }^{1} \mathrm{H}$ NMR $(400 \mathrm{MHz}$, Chloroform- $d) \delta 9.54(\mathrm{~s}, 1 \mathrm{H}, \mathrm{NH}), 7.36(\mathrm{~d}, J=6.1 \mathrm{~Hz}, 1 \mathrm{H}$, F-uracil C=C$\mathrm{H}), 5.28\left(\mathrm{t}, J=9.0 \mathrm{~Hz}, 1 \mathrm{H}\right.$, cyclobutane- $\mathrm{CH}$ ), $3.78\left(\mathrm{~s}, 3 \mathrm{H}\right.$, ester $\left.\mathrm{CH}_{3}\right), 3.73\left(\mathrm{~s}, 3 \mathrm{H}\right.$, ester $\left.\mathrm{CH}_{3}\right)$, $2.68-2.61\left(\mathrm{~m}, 2 \mathrm{H}\right.$, cyclobutane- $\left.\mathrm{CH}_{2}\right), 2.41-2.32\left(\mathrm{~m}, 1 \mathrm{H}\right.$, cyclobutane- $\left.\mathrm{CH}_{2}\right), 2.32-2.25(\mathrm{~m}$, $1 \mathrm{H}$, cyclobutane- $\mathrm{CH}_{2}$ ).

${ }^{13} \mathrm{C}$ NMR $(101 \mathrm{MHz}$, Chloroform- $d) \delta 169.7,168.8,156.8(\mathrm{~d}, J=26.7 \mathrm{~Hz}), 149.7,140.2(\mathrm{~d}, J=$ $237.5 \mathrm{~Hz}$ ), 126.3 (d, $J=33.7 \mathrm{~Hz}), 59.0,55.2,53.3,53.3,23.7,23.0$.

IR: 3211 (w), 3085 (w), 2960 (w), 2362 (w), 2336 (w), 1726 (s), 1439 (w), $1361(w), 1273$ $(\mathrm{m}), 1110(\mathrm{w}), 915(\mathrm{w}), 735(\mathrm{~m})$.

HRMS (ESI) calcd for $\mathrm{C}_{12} \mathrm{H}_{13} \mathrm{FN}_{2} \mathrm{NaO}_{6}{ }^{+}[\mathrm{M}+\mathrm{Na}]^{+} 323.0650$; found 323.0654 .

Dimethyl 2-(3-benzoyl-5-fluoro-2,4-dioxo-3,4-dihydropyrimidin-1(2H)-yl)cyclobutane1,1-dicarboxylate (18b).
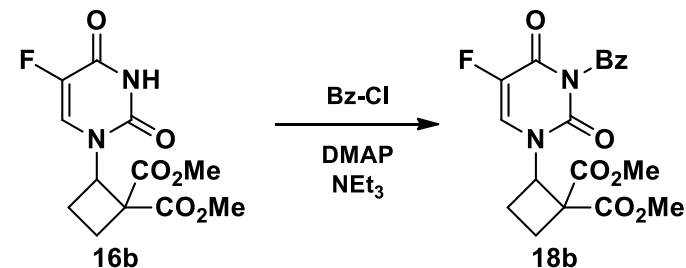

In a flame dried microwave vial under nitrogen atmosphere, $16 \mathrm{~b}(0.70 \mathrm{~g}, 2.3 \mathrm{mmol}, 1.0$ equiv $)$ and dimethyl-4-aminopyridin (0.28 g, $2.3 \mathrm{mmol}, 1.0$ equiv) were dissolved in dry dichloromethane $(12 \mathrm{~mL})$. Triethylamine $(1.3 \mathrm{~mL}, 9.3 \mathrm{mmol}, 4.0$ equiv) was added and the solution was cooled to $0^{\circ} \mathrm{C}$. Then, benzoyl chloride $(0.81 \mathrm{~mL}, 6.9 \mathrm{mmol}, 3.0$ equiv) was added dropwise and the reaction mixture was stirred at room temperature for 16 hours. A saturated solution of $\mathrm{NaHCO}_{3}(8 \mathrm{~mL})$ was added to the reaction mixture and the layer were separated. The aqueous layer was extracted with dichloromethane $(2 \times 20 \mathrm{~mL})$. The combined organic layers were washed with $1 \mathrm{M} \mathrm{HCl}(2 \times 40 \mathrm{~mL})$ and with water $(30 \mathrm{~mL})$, dried over $\mathrm{MgSO}_{4}$ and concentrated under reduce pressure. The pure product $18 \mathrm{~b}(0.61 \mathrm{~g}, 1.5 \mathrm{mmol}, 65 \%)$ was obtained as colorless foam.

RF $($ Pent/AcOEt $(4: 6))=0.55$.

Mp: $60.9-62.5^{\circ} \mathrm{C}$

${ }^{1}$ H NMR (400 MHz, Chloroform- $\left.d\right) \delta 7.93-7.89$ (m, 2H, Ar-H), $7.66-7.59$ (m, 1H, Ar-H), 7.50 - $7.43(\mathrm{~m}, 3 \mathrm{H}, \mathrm{Ar}-\mathrm{H}$ and F-uracil $\mathrm{C}=\mathrm{C}-\mathrm{H}), 5.12(\mathrm{t}, J=9.4 \mathrm{~Hz}, 1 \mathrm{H}$, cyclobutane-CH), 3.69 (s, $3 \mathrm{H}$, ester $\mathrm{CH}_{3}$ ), $3.64\left(\mathrm{~s}, 3 \mathrm{H}\right.$, ester $\mathrm{CH}_{3}$ ), 2.73 (q, $J=10.0 \mathrm{~Hz}, 1 \mathrm{H}$, cyclobutane- $\mathrm{CH}_{2}$ ), $2.66-$ $2.58\left(\mathrm{~m}, 1 \mathrm{H}\right.$, cyclobutane- $\mathrm{CH}_{2}$ ), $2.33\left(\mathrm{dtd}, J=10.6,8.4,2.4 \mathrm{~Hz}, 1 \mathrm{H}\right.$, cyclobutane- $\mathrm{CH}_{2}$ ), 2.23 (dt, $J=11.6,8.9 \mathrm{~Hz}, 1 \mathrm{H}$, cyclobutane- $\mathrm{CH}_{2}$ ). 
${ }^{13} \mathrm{C}$ NMR (101 MHz, Chloroform- $d$ ) $\delta 169.8,168.7,167.1,156.3(\mathrm{~d}, J=27.1 \mathrm{~Hz}), 148.6,139.8$ (d, $J=239.4 \mathrm{~Hz}$ ), 135.5, 131.0, 130.9, 129.3, 127.1 (d, $J=33.6 \mathrm{~Hz}$ ), 58.7, 56.8, 53.4, 53.3, 23.9, 22.8 .

IR: 3090 (w), 3009 (w), 2958 (w), 2361 (w), 2262 (w), 1723 (s), 1668 (s), 1598 (w), 1445 (m), $1357(w), 1266(s), 1106(m), 982(w), 912(m), 851(w), 732(s)$.

HRMS (ESI) calcd for $\mathrm{C}_{19} \mathrm{H}_{17} \mathrm{~N}_{2} \mathrm{FNaO}_{7}{ }^{+}[\mathrm{M}+\mathrm{Na}]^{+} 427.0917$; found 427.0920.

\subsection{Purine cyclopropanes.}

Dimethyl 2-(6-amino-9H-purin-9-yl)cyclopropane-1,1-dicarboxylate (38a).

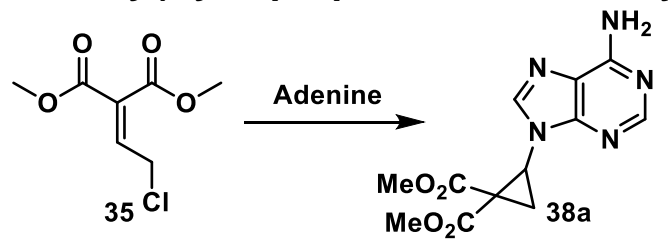

Following the procedure described by Geen et al., ${ }^{6}$ dry $\mathrm{K}_{2} \mathrm{CO}_{3}(2.0 \mathrm{~g}, 14 \mathrm{mmol}, 1.5$ equiv), dimethyl 2-(2-chloroethylidene)malonate (35) $(2.0 \mathrm{~g}, 10.5 \mathrm{mmol}, 1.1$ equiv), adenine (1.3 g, 9.5 $\mathrm{mmol}, 1$ equiv) were stirred in a flame dried flask under nitrogen in dry DMF $(25 \mathrm{~mL})$ for $16 \mathrm{~h}$. The reaction mixture was filtered on cotton with DMF $(10 \mathrm{~mL})$ and concentrated under high vacuo. The residue was purified by column chromatography using a mixture of pentane AcOEt (from 1:1 to 7:3 AcOEt/pentane) to afford the pure cyclopropane $38 \mathrm{a}(2.2 \mathrm{~g}, 7.3 \mathrm{mmol}, 69 \%$ yield) as a white powder.

RF $(\mathrm{MeOH} / \mathrm{DCM}(0.5: 9.5)=0.39$.

Mp 199.2-202. ${ }^{\circ} \mathrm{C}$. (Decomposition)

${ }^{1} \mathrm{H}$ NMR $\left(400 \mathrm{MHz}\right.$, DMSO- $\left.d_{6}\right) \delta 8.17$ (s, $1 \mathrm{H}$, Adenine $\mathrm{CH}$ ), 8.15 (s, $1 \mathrm{H}$, Adenine $\mathrm{CH}$ ), 7.27 (s, $2 \mathrm{H}, \mathrm{NH}_{2}$ ), 4.48 (dd, $J=8.5,6.3 \mathrm{~Hz}, 1 \mathrm{H}$ cyclopropane $\mathrm{NCH}$ ), 3.77 (s, 3H, Me ester), 3.31 (s, $3 \mathrm{H}$, Me ester), 2.85 (t, $J=6.5 \mathrm{~Hz}, 1 \mathrm{H}$, cyclopropane $\left.\mathrm{CH}_{2}\right), 2.19-2.05(\mathrm{~m}, 1 \mathrm{H}$, cyclopropane $\mathrm{CH}_{2}$ ).

${ }^{13} \mathrm{C}$ NMR (101 MHz, DMSO) $\delta 168.2,165.9,156.4,152.9,150.9,140.9,119.1,53.4,52.9$, $37.9,35.2,18.4$.

IR 3434 (w), 3083 (w), 3001 (w), 1719 (s), 1602 (s), 1431 (m), 1299 (s), 1200 (s), 893 (w), 768 (w).

HRMS (ESI) calcd for $\mathrm{C}_{12} \mathrm{H}_{14} \mathrm{~N}_{5} \mathrm{O}_{4}{ }^{+}[\mathrm{M}+\mathrm{H}]+2$ 292.1040; found 292.1045

Dimethyl 2-(2-amino-6-chloro-9H-purin-9-yl)cyclopropane-1,1-dicarboxylate (38b).<smiles>COC(=O)C(=CCCl)C(=O)OC</smiles>

Following the procedure described by Geen et al. ${ }^{6}$, dry $\mathrm{K}_{2} \mathrm{CO}_{3}(3.42 \mathrm{~g}, 24.8 \mathrm{mmol}, 1.5$ equiv), dimethyl 2-(2-chloroethylidene)malonate (35) (3.50 g, $18.2 \mathrm{mmol}, 1.1$ equiv) and 6-chloro-7H-

${ }^{6}$ G. R. Geen, P. M. Kincey, B. M. Choudary, Tetrahedron Lett. 1992, 33, 4609-4612. 
purin-2-amine ( $2.80 \mathrm{~g}, 1.5 \mathrm{mmol}, 1$ equiv) were stirred in a flame dried flask under nitrogen in dry DMF (28 $\mathrm{mL})$ for $16 \mathrm{~h}$. The reaction mixture was filtered on cotton with DMF $(10 \mathrm{~mL})$ and concentrated under high vacuo. The residue was purified by column chromatography using a mixture of dichloromethane methanol (from pure dichloromethane to 8:2 dichloromethane/methanol) to afford the pure cyclopropane $38 \mathrm{~b}(4.30 \mathrm{~g}, 13.2 \mathrm{mmol}, 51 \%$ yield) as a white powder.

$\mathbf{R F}(\mathrm{MeOH} / \mathrm{DCM}(1: 9)=0.56$.

MP 215.3-218.6 ${ }^{\circ} \mathrm{C}$. (Decomposition)

${ }^{1} \mathrm{H}$ NMR $\left(400 \mathrm{MHz}, \mathrm{DMSO}-d_{6}\right) \delta 8.16(\mathrm{~s}, 1 \mathrm{H}$, purine $\mathrm{CH}), 7.05\left(\mathrm{~s}, 2 \mathrm{H}, \mathrm{NH}_{2}\right), 4.41$ (dd, $J=8.6$, $6.3 \mathrm{~Hz}, 1 \mathrm{H}$, cyclopropane NCH), 3.38 (s, 3H, Me ester), 3.34 (s, 3H, Me ester), 2.82 (t, J = 6.5 $\mathrm{Hz}, 1 \mathrm{H}$, cyclopropane $\mathrm{CH}_{2}$ ), 2.10 (dd, $J=8.6,6.7 \mathrm{~Hz}, 1 \mathrm{H}$, cyclopropane $\mathrm{CH}_{2}$ ).

${ }^{13} \mathrm{C}$ NMR (101 MHz, DMSO) $\delta 168.1,165.8,160.4,155.4,149.8,143.0,123.6,53.5,53.1$, $37.8,35.2,18.5$.

IR 3436 (w), 3163 (w), 1725 (s), 1563 (s), 1288 (s), 1210 (s), 1108 (s), 911 (s), 787 (w), 716 (m).

HRMS (ESI) calcd for $\mathrm{C}_{12} \mathrm{H}_{13} \mathrm{CIN}_{5} \mathrm{O}_{4}{ }^{+}[\mathrm{M}+\mathrm{H}]^{+} 326.0651$; found 326.0654 .

Dimethyl 2-(6-chloro-9H-purin-9-yl)cyclopropane-1,1-dicarboxylate (38c).

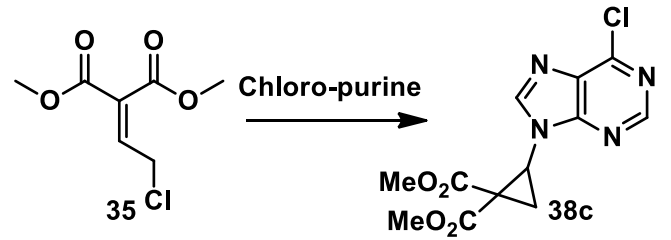

Following the procedure described by Geen et al., ${ }^{6}$ dry $\mathrm{K}_{2} \mathrm{CO}_{3}(5.7 \mathrm{~g}, 41 \mathrm{mmol}, 1.5$ equiv), dimethyl 2-(2-chloroethylidene)malonate (35) $(5.8 \mathrm{~g}, 30 \mathrm{mmol}, 1.1$ equiv) and 6-chloro-purine (4.22 g, $27.3 \mathrm{mmol}, 1$ equiv) were stirred in a flame dried flask under nitrogen in dry DMF (55 $\mathrm{mL})$ for $16 \mathrm{~h}$. The reaction mixture was filtered on cotton with DMF (10 mL) and concentrated under high vacuo. The residue was purified by column chromatography using a mixture of dichloromethane methanol (from pure dichloromethane to 8:2 dichloromethane/methanol) to afford the pure cyclopropane $38 \mathrm{c}(2.5 \mathrm{~g}, 8.1 \mathrm{mmol}, 30 \%$ yield $)$ as foam.

RF $($ AcOEt/pentane $(7: 3)=0.31$

MP $120.1-123.6^{\circ} \mathrm{C}$.

${ }^{1} \mathrm{H}$ NMR $(400 \mathrm{MHz}$, Chloroform- $d) \delta 8.77(\mathrm{~s}, 1 \mathrm{H}$, purine $\mathrm{CH}), 8.10(\mathrm{~s}, 1 \mathrm{H}$, purine $\mathrm{CH}), 4.49$ (dd, $J=8.4,6.2 \mathrm{~Hz}, 1 \mathrm{H}$, cyclopropane $\mathrm{NCH}$ ), 3.87 (s, 3H, Me ester), 3.47 (s, 3H, Me ester), 2.82 (t, $J=6.4 \mathrm{~Hz}, 1 \mathrm{H}$, cyclopropane $\left.\mathrm{CH}_{2}\right), 2.18\left(\mathrm{dd}, J=8.4,6.7 \mathrm{~Hz}, 1 \mathrm{H}\right.$, cyclopropane $\mathrm{CH}_{2}$ ).

${ }^{13} \mathrm{C}$ NMR $\left(101 \mathrm{MHz}, \mathrm{CDCl}_{3}\right) \delta 167.7,165.4,152.6,152.5,151.4,145.1,131.5,53.5,53.3,37.7$, 35.0, 19.2 .

IR 2938.34 (w), 1729 (s), 1603 (w), 1333 (s), 1230 (s), 1204 (s), 1142 (s), 941 (m).

HRMS (ESI) calcd for $\mathrm{C}_{12} \mathrm{H}_{12} \mathrm{CIN}_{4} \mathrm{O}_{4}{ }^{+}[\mathrm{M}+\mathrm{H}]^{+} 311.0542$; found 311.0542 .

Dimethyl 2-(6-amino-9H-purin-9-yl)cyclopropane-1,1-dicarboxylate (38d). 


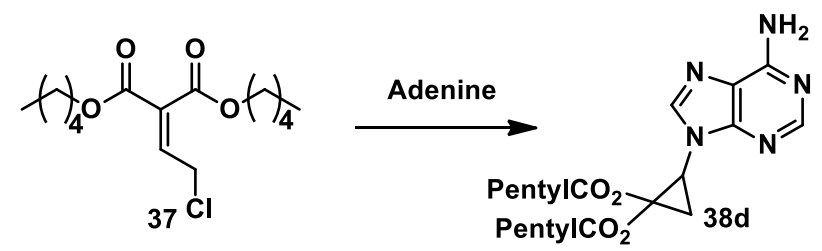

Following the procedure described by Geen et al. ${ }^{6}$, dry $\mathrm{K}_{2} \mathrm{CO}_{3}(1.2 \mathrm{~g}, 8.9 \mathrm{mmol}, 1.5$ equiv), dimethyl 2-(2-chloroethylidene)pentylmalonate $(37)(3.0 \mathrm{~g}, 6.5 \mathrm{mmol}, 1.1$ equiv, $66 \% \mathrm{Wt})$ and adenine $(0.80 \mathrm{~g}, 5.9 \mathrm{mmol}$, 1 equiv) were stirred in a flame dried flask under nitrogen in dry DMF $(11 \mathrm{~mL})$ for $16 \mathrm{~h}$. The reaction mixture was filtered on cotton with DMF $(5 \mathrm{~mL})$ and concentrated under high vacuo. The residue was purified by column chromatography using a mixture of dichloromethane methanol (from pure dichloromethane to 9.8:0.2 dichloromethane/methanol) to afford the pure cyclopropane $38 \mathbf{d}(2.22 \mathrm{~g}, 5.49 \mathrm{mmol}, 93 \%$ yield) as a white sticky powder.

$\mathbf{R F}(\mathrm{MeOH} / \mathrm{DCM}(0.2: 9.8)=0.51$.

Mp 68.2-70.0 ${ }^{\circ} \mathrm{C}$.

${ }^{1} \mathrm{H}$ NMR $\left(400 \mathrm{MHz}, \mathrm{DMSO}-d_{6}\right) \delta 8.15(\mathrm{~s}, 2 \mathrm{H}$, adenine $\mathrm{CH}), 7.26\left(\mathrm{~s}, 2 \mathrm{H}\right.$, adenine $\left.\mathrm{NH}_{2}\right), 4.46$ (dd, $J=8.6,6.3 \mathrm{~Hz}, 1 \mathrm{H}$, cyclopropane $\mathrm{NCH}), 4.27-4.18\left(\mathrm{~m}, 2 \mathrm{H}\right.$, pentyl $\left.\mathrm{CH}_{2}\right), 3.70(\mathrm{td}, J=6.4$, $4.6 \mathrm{~Hz}, 2 \mathrm{H}$, pentyl $\mathrm{CH}_{2}$ ), $2.86\left(\mathrm{t}, J=6.5 \mathrm{~Hz}, 2 \mathrm{H}\right.$, cyclopropane $\mathrm{CH}_{2}$ ), 2.05 (dd, $J=8.7,6.7 \mathrm{~Hz}$, $2 \mathrm{H}$, cyclopropane $\mathrm{CH}_{2}$ ), $1.71-1.49\left(\mathrm{~m}, 2 \mathrm{H}\right.$, pentyl $\left.\mathrm{CH}_{2}\right), 1.31$ (ddt, $J=18.8,7.9,4.0 \mathrm{~Hz}, 4 \mathrm{H}$, pentyl $\mathrm{CH}_{2}$ ), $1.02-0.81\left(\mathrm{~m}, 5 \mathrm{H}\right.$, pentyl $\mathrm{CH}_{2}$ and pentyl $\left.\mathrm{CH}_{3}\right), 0.78(\mathrm{t}, J=7.3 \mathrm{~Hz}, 3 \mathrm{H}$, pentyl $\left.\mathrm{CH}_{3}\right)$.

${ }^{13} \mathrm{C}$ NMR (101 MHz, DMSO) $\delta 167.9,165.4,156.4,153.1,151.0,140.6,119.2,66.0,65.7$, $37.8,35.7,28.1,27.9,27.7,27.5,22.2,22.0,18.0,14.3,14.1$.

IR 3312 (w), 3135 (w), 2958 (m), 1726 (s), 1601 (m), 1471 (m), 1294 (s), 1197 (s), 967 (m), $735(\mathrm{~m})$.

HRMS (ESI) calcd for $\mathrm{C}_{20} \mathrm{H}_{30} \mathrm{~N}_{5} \mathrm{O}_{4}{ }^{+}[\mathrm{M}+\mathrm{H}]^{+}$404.2292; found 404.2300.

Dipentyl 2-(2-amino-6-chloro-9H-purin-9-yl)cyclopropane-1,1-dicarboxylate (38e).<smiles>CCOC(=O)C(=CCCl)C(=O)OC</smiles>

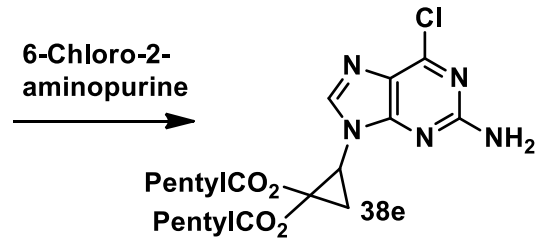

Following the procedure described by Geen et al. ${ }^{6}$, dry $\mathrm{K}_{2} \mathrm{CO}_{3}(0.71 \mathrm{~g}, 5.1 \mathrm{mmol}, 1.5$ equiv), dimethyl 2-(2-chloroethylidene)pentylmalonate (37) (2.87 g, $3.77 \mathrm{mmol}, 1.1$ equiv, $40 \% \mathrm{Wt}$ ) and 6-chloro-7H-purin-2-amine $(0.58 \mathrm{~g}, 3.4 \mathrm{mmol}, 1$ equiv) were stirred in a flame dried flask under nitrogen in dry DMF $(7 \mathrm{~mL})$ for $16 \mathrm{~h}$. The reaction mixture was filtered on cotton with DMF (2 mL) and concentrated under high vacuo. The residue was purified by column chromatography using a mixture of dichloromethane methanol (from pure dichloromethane to 9.8:0.2 dichloromethane/methanol) to afford the pure cyclopropane $38 \mathrm{e}(1.35 \mathrm{~g}, 3.08 \mathrm{mmol}$, $90 \%$ yield) as a white powder.

RF $(\mathrm{MeOH} / \mathrm{DCM}(0.5: 9.5)=0.47$.

Mp 127.1-128.3 ${ }^{\circ} \mathrm{C}$. (Decomposition) 
${ }^{1} \mathrm{H}$ NMR $(400 \mathrm{MHz}$, Chloroform- $d) \delta 8.13(\mathrm{~s}, 1 \mathrm{H}$, purine $\mathrm{CH}), 7.04\left(\mathrm{~s}, 2 \mathrm{H}\right.$, purine $\left.\mathrm{NH}_{2}\right), 4.37$ (dd, $J=8.6,6.3 \mathrm{~Hz}, 1 \mathrm{H}$, cyclopropane $\mathrm{NCH}$ ), $4.26-3.99\left(\mathrm{~m}, 2 \mathrm{H}\right.$, pentyl $\left.\mathrm{CH}_{2}\right), 3.75(\mathrm{td}, J=6.5$, $2.5 \mathrm{~Hz}, 2 \mathrm{H}$, pentyl $\mathrm{CH}_{2}$ ), 2.82 (t, $J=6.5 \mathrm{~Hz}, 1 \mathrm{H}$, cyclopropane $\mathrm{CH}_{2}$ ), 2.01 (dd, $J=8.6,6.8 \mathrm{~Hz}$, $1 \mathrm{H}$, cyclopropane $\mathrm{CH}_{2}$ ), $1.61\left(\mathrm{dd}, J=9.2,4.9 \mathrm{~Hz}, 2 \mathrm{H}\right.$, pentyl $\left.\mathrm{CH}_{2}\right), 1.31(\mathrm{~h}, J=3.7 \mathrm{~Hz}, 4 \mathrm{H}$, pentyl $\left.\mathrm{CH}_{2}\right), 1.10\left(\mathrm{dq}, J=14.3,7.3,6.6 \mathrm{~Hz}, 4 \mathrm{H}\right.$, pentyl $\left.\mathrm{CH}_{2}\right), 0.99-0.81\left(\mathrm{~m}, 5 \mathrm{H}\right.$, pentyl $\mathrm{CH}_{2}$ and pentyl $\mathrm{CH}_{3}$ ), $0.77\left(\mathrm{t}, J=7.3 \mathrm{~Hz}, 3 \mathrm{H}\right.$, pentyl $\mathrm{CH}_{3}$ ).

${ }^{13} \mathrm{C}$ NMR $\left(101 \mathrm{MHz}, \mathrm{CDCl}_{3}\right) \delta 172.7,170.1,165.1,160.2,154.6,147.4,128.5,70.9,70.6,42.4$, $40.5,32.8,32.7,32.5,32.3,26.9,26.8,22.7,19.0,18.8$.

IR 3441 (w), 3305 (w), 3201 (w), 2955 (w), 1699 (m), 1641 (s), 1560 (m), 1479 (w), 1309 (s), $1222(\mathrm{~s}), 1186(\mathrm{~m}), 959(\mathrm{~m}), 912(\mathrm{~m}), 785(\mathrm{w})$.

HRMS (ESI) calcd for $\mathrm{C}_{20} \mathrm{H}_{28} \mathrm{CIN}_{5} \mathrm{NaO}_{4}[\mathrm{M}+\mathrm{Na}] 460.1727$; found 460.1727.

Dipentyl 2-(6-chloro-9H-purin-9-yl)cyclopropane-1,1-dicarboxylate (38f).<smiles>CCOC(=O)C(=CCCl)C(=O)OC</smiles><smiles>COC(=O)OC1(O)C(CC(C)C)C1n1cnc2c(Cl)ncnc21</smiles>

Following the procedure described by Geen et al. ${ }^{6}$, dry $\mathrm{K}_{2} \mathrm{CO}_{3}(1.99 \mathrm{~g}, 14.4 \mathrm{mmol}, 1.5$ equiv), dimethyl 2-(2-chloroethylidene)pentylmalonate (37) $(6.5 \mathrm{~g}, 11 \mathrm{mmol}, 1.1$ equiv, $50 \% \mathrm{Wt})$ and 6-chloro-purine (1.5 g, $9.6 \mathrm{mmol}, 1$ equiv) were stirred in a flame dried flask under nitrogen in dry DMF $(20 \mathrm{~mL})$ for $16 \mathrm{~h}$. The reaction mixture was filtered on cotton with DMF $(10 \mathrm{~mL})$ and concentrated under high vacuo. The residue was purified by column chromatography using a mixture of AcOEt pentane (from 1:1 to 7:3 AcOEt/pentane) to afford the pure cyclopropane $\mathbf{3 8 f}$ $(2.5 \mathrm{~g}, 5.9 \mathrm{mmol}, 62 \%$ yield) as a yellow oil.

$\mathbf{R F}$ (pentane/AcOEt $(8: 2)=0.18$.

${ }^{1} \mathrm{H}$ NMR $(400 \mathrm{MHz}$, Chloroform- $d$ ) $\delta 8.80(\mathrm{~s}, 1 \mathrm{H}$, purine $\mathrm{CH}), 8.12(\mathrm{~s}, 1 \mathrm{H}$, purine $\mathrm{CH}), 4.52$ (dd, $J=8.4,6.2 \mathrm{~Hz}, 1 \mathrm{H}$, cyclopropane $\mathrm{NCH}$ ), $4.32\left(\mathrm{dt}, J=10.8,6.8 \mathrm{~Hz}, 1 \mathrm{H}\right.$, pentyl $\mathrm{CH}_{2}$ ), 4.25 (dt, $J=10.7,6.7 \mathrm{~Hz}, 1 \mathrm{H}$, pentyl $\mathrm{CH}_{2}$ ), 3.87 (ddt, $J=33.9,10.8,6.7 \mathrm{~Hz}, 2 \mathrm{H}$, pentyl $\mathrm{CH}_{2}$ ), 2.84 (t, $J$ $=6.4 \mathrm{~Hz}, 1 \mathrm{H}$, cyclopropane $\left.\mathrm{CH}_{2}\right), 2.17\left(\mathrm{dd}, J=8.4,6.6 \mathrm{~Hz}, 1 \mathrm{H}\right.$, cyclopropane $\left.\mathrm{CH}_{2}\right), 1.44-$ $1.26\left(\mathrm{~m}, 4 \mathrm{H}\right.$, pentyl $\left.\mathrm{CH}_{2}\right), 1.26-1.17\left(\mathrm{~m}, 2 \mathrm{H}\right.$, pentyl $\left.\mathrm{CH}_{2}\right), 1.14-1.04\left(\mathrm{~m}, 2 \mathrm{H}\right.$, pentyl $\left.\mathrm{CH}_{2}\right)$, $0.99-0.90\left(\mathrm{~m}, 5 \mathrm{H}\right.$, pentyl $\mathrm{CH}_{2}$ and pentyl $\left.\mathrm{CH}_{3}\right), 0.85\left(\mathrm{t}, J=7.3 \mathrm{~Hz}, 3 \mathrm{H}\right.$, pentyl $\left.\mathrm{CH}_{3}\right)$.

${ }^{13} \mathrm{C}$ NMR $\left(101 \mathrm{MHz}, \mathrm{CDCl}_{3}\right) \delta 167.5,165.0,152.7,152.4,151.3,145.0,131.5,66.7,66.5,37.5$, $35.52,28.1,27.9,27.8,27.6,22.2,22.1,18.8,13.9,13.8$.

IR 2953 (w), 2866 (w), 1723 (s), 1587 (w), 1563 (m), 1284 (s), 1201 (s), 1134 (s), 958 (m).

HRMS (ESI) calcd for $\mathrm{C}_{20} \mathrm{H}_{28} \mathrm{CIN}_{4} \mathrm{O}_{4}{ }^{+}[\mathrm{M}+\mathrm{H}]^{+}$423.1794; found 423.1794.

Dimethyl 2-(2-ditButylcarboxylate-amino-6-chloro-9H-purin-9-yl)cyclopropane-1,1dicarboxylate (39).<smiles>COC(=O)C(C)C1CC1n1cnc2c(Cl)nc(N(C(=O)OC(C)(C)C)C(=O)c3ccccc3)nc21</smiles> 
Cyclopropane 38b $(0.050 \mathrm{~g}, 0.15 \mathrm{mmol}, 1.0$ equiv), di-tert-butyl dicarbonate $(0.14 \mathrm{~g}, 0.66$ mmol, 4.3 equiv) and dimethylaminopyridine (1.8 $\mathrm{mg}, 0.015 \mathrm{mmol}, 0.1$ equiv) were stirred in acetonitrile $(2.5 \mathrm{~mL})$ for $5 \mathrm{~h}$ in a flame-dried flask under nitrogen. After removal of the solvent, EtOAc $(10 \mathrm{~mL})$ was added and the solution was washed with $1 \mathrm{~N} \mathrm{HCl}(3 \mathrm{~mL})$ and then with brine $(3 \times 10 \mathrm{~mL})$. Then the mixture was dried over anhydrous $\mathrm{MgSO}_{4}$, filtered and concentrated. Then the crude residue was purified by column chromatography using a mixture of pentane and ethyl acetate (from 90:10 to 70:30 pentane/ ethyl acetate) affording the pure product $39(0.069 \mathrm{~g}, 0.13 \mathrm{mmol}, 85 \%$ yield, $90 \%$ purity) as a slightly yellow sticky oil.

RF (Pent/AcOEt $(7: 3)=0.41$.

${ }^{1} \mathrm{H}$ NMR $(400 \mathrm{MHz}$, Chloroform- $d$ ) $\delta 8.04(\mathrm{~s}, 1 \mathrm{H}$, purine $\mathrm{CH}), 4.38(\mathrm{dd}, J=8.4,6.1 \mathrm{~Hz}, 1 \mathrm{H}$, cyclopropane $\mathrm{NCH}$ ), 3.78 (s , 3H, Me ester), 3.43 (s, 3H, Me ester), 2.73 (t, $J=6.4 \mathrm{~Hz}, 1 \mathrm{H}$, cyclopropane $\left.\mathrm{CH}_{2}\right), 2.10$ (m, 1H, cyclopropane $\left.\mathrm{CH}_{2}\right), 1.39(\mathrm{~m}, 18 \mathrm{H}, \mathrm{Boc})$.

${ }^{13} \mathrm{C}$ NMR $\left(101 \mathrm{MHz}, \mathrm{CDCl}_{3}\right) \delta 167.6,165.2,153.4,152.3,151.4,150.4,145.8,129.7,83.7$, 53.4, 53.2, 37.6, 35.0, 27.8, 19.1 .

Boc carbon peaks are superposed (Methyls, quaternary carbons and $\mathrm{C}=\mathrm{O}$.)

IR 3546 (w), 3117 (w), $2981(w), 1792(\mathrm{~m}), 1730(\mathrm{~s}), 1568(\mathrm{~m}), 1280(\mathrm{~s}), 1103(\mathrm{~s}), 856(\mathrm{~m})$, $779(\mathrm{~m})$.

HRMS (ESI) calcd for $\mathrm{C}_{22} \mathrm{H}_{28} \mathrm{CIN}_{5} \mathrm{NaO}_{8}{ }^{+}[\mathrm{M}+\mathrm{Na}]^{+}$548.1519; found 548.1515.

\subsection{Dipolarophiles.}

Triisopropyl((1-phenylvinyl)oxy)silane (21).

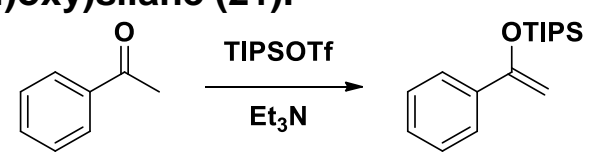

20

21

Acetophenone (20) (1.0 g, $8.3 \mathrm{mmol}, 1$ equiv) was solubilized in DCM (8 mL) and triethylamine $\left(1.73 \mathrm{~mL}, 12.5 \mathrm{mmol}, 1.5\right.$ equiv) was added at room temperature. Then at $0{ }^{\circ} \mathrm{C}$ triisopropylsilyl trifluoromethanesulfonate $(2.7 \mathrm{~mL}, 10 \mathrm{mmol}, 1.2$ equiv) was added dropwise and the reaction mixture was stirred at room temperature for $8 \mathrm{~h}$. The solvent was evaporated under a flow of nitrogen. The crude product was purified by column chromatography, eluting with pentane and $1 \% \mathrm{NEt}_{3}$ to obtain the pure enol-ether $21(2.0 \mathrm{~g}, 7.2 \mathrm{mmol}, 86 \%$ yield) as a colorless oil.

${ }^{1} \mathrm{H}$ NMR (400 MHz, Chloroform- $\left.d\right) \delta 7.70-7.64(\mathrm{~m}, 2 \mathrm{H}, \mathrm{Ar}-\mathrm{H}), 7.41-7.27(\mathrm{~m}, 3 \mathrm{H}, \mathrm{Ar}-\mathrm{H}), 4.87$ $\left(\mathrm{d}, J=1.8 \mathrm{~Hz}, 1 \mathrm{H}, \mathrm{C}=\mathrm{CH}_{2}\right), 4.44\left(\mathrm{~d}, J=1.9 \mathrm{~Hz}, 1 \mathrm{H}, \mathrm{C}=\mathrm{CH}_{2}\right), 1.33(\mathrm{~m}, 3 \mathrm{H}, \mathrm{TIPS}-\mathrm{CH}), 1.16(\mathrm{~m}$, $\left.18 \mathrm{H}, \mathrm{TIPS}-\mathrm{CH}_{3}\right)$.

${ }^{1} \mathrm{H}$ NMR values correspond to the literature. ${ }^{1}$

((1-(4-Fluorophenyl)vinyl)oxy)triisopropylsilane (49).<smiles>C=C(O[SnH3])c1ccc(F)cc1</smiles>

(4-Fluorophenyl)ethanone (48) $(0.44 \mathrm{~mL}, 3.6 \mathrm{mmol}$, 1equiv) was solubilized in THF (15 mL) at $-78{ }^{\circ} \mathrm{C}$ and NaHMDS (2M solution in THF, $2.1 \mathrm{~mL}, 4.1 \mathrm{mmol}, 1.1$ equiv) was added dropwise. The mixture was stirred 1 hour at room temperature and cooled down to $-78^{\circ} \mathrm{C}$. TIPS-Cl $(0.86$ $\mathrm{mL}, 4.1 \mathrm{mmol}, 1.1$ equiv) was added dropwise and the reaction mixture was stirred at room temperature for $8 \mathrm{~h}$. The solvent was evaporated and the crude product was purified by column 
chromatography, eluting with pentane and $1 \% \mathrm{NEt}_{3}$ to obtain the pure enol-ether $49(0.62 \mathrm{~g}$, $2.1 \mathrm{mmol}, 58 \%$ yield) as a slightly yellow oil.

${ }^{1} \mathrm{H}$ NMR $(400 \mathrm{MHz}$, Chloroform-d) $\delta 7.61$ (dd, $J=8.9,5.4 \mathrm{~Hz}, 2 \mathrm{H}, \mathrm{Ar}-\mathrm{H}), 7.00(\mathrm{t}, J=8.8 \mathrm{~Hz}$, $2 \mathrm{H}, \mathrm{Ar}-\mathrm{H}), 4.77(\mathrm{~d}, J=1.9 \mathrm{~Hz}, 1 \mathrm{H}, \mathrm{C}=\mathrm{CH}), 4.39(\mathrm{~d}, J=1.9 \mathrm{~Hz}, 1 \mathrm{H}, \mathrm{C}=\mathrm{CH}), 1.39-1.20(\mathrm{~m}$, $3 \mathrm{H}$, TIPS-CH), 1.34-1.12 (m, 18H, TIPS- $\left.\mathrm{CH}_{3}\right)$.

${ }^{1} \mathrm{H}$ NMR values correspond to the literature. ${ }^{1}$

(E)-Triisopropyl((4-phenylbuta-1,3-dien-2-yl)oxy)silane (51).

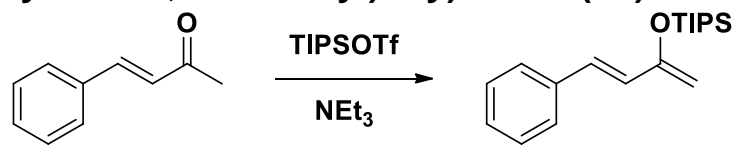

50

51

(E)-4-Phenylbut-3-en-2-one (50) (0.42 g, $2.9 \mathrm{mmol}, 1$ equiv) was solubilized in DCM ( $3 \mathrm{~mL})$ and triethylamine $(0.60 \mathrm{~mL}, 4.3 \mathrm{mmol}, 1.5$ equiv) was added at room temperature. Then triisopropylsilyl trifluoromethanesulfonate $\left(0.93 \mathrm{~mL}, 3.4 \mathrm{mmol}, 1.2\right.$ equiv) was added at $0{ }^{\circ} \mathrm{C}$ dropwise and the reaction mixture was stirred at room temperature for $8 \mathrm{~h}$. The solvent was evaporated under a flow of nitrogen. The crude product was purified by column chromatography, eluting with pentane and $1 \% \mathrm{NEt}_{3}$ to obtain the pure enol ether $\mathbf{5 1}(0.87 \mathrm{~g}$, $2.9 \mathrm{mmol}$, quantitative yield) as a colorless oil.

$\mathbf{R F}($ pentane $)=0.88$.

${ }^{1}$ H NMR (400 MHz, Chloroform- $d$ ) $\delta 7.64-7.54(\mathrm{~m}, 2 \mathrm{H}, \mathrm{Ar}-\mathrm{H}), 7.45(\mathrm{~m}, 2 \mathrm{H}, \mathrm{Ar}-\mathrm{H}), 7.40-7.32$ $(\mathrm{m}, 1 \mathrm{H}, \mathrm{Ar}-\mathrm{H}), 7.15(\mathrm{~d}, J=15.6 \mathrm{~Hz}, 1 \mathrm{H}, \mathrm{C}=\mathrm{CH}), 6.74(\mathrm{~d}, J=15.7 \mathrm{~Hz}, 1 \mathrm{H}, \mathrm{C}=\mathrm{CH}), 4.61-4.59$ $\left(\mathrm{s}, 1 \mathrm{H}, \mathrm{C}=\mathrm{CH}_{2}\right), 4.58\left(\mathrm{~s}, 1 \mathrm{H}, \mathrm{C}=\mathrm{CH}_{2}\right), 1.54-1.42(\mathrm{~m}, 3 \mathrm{H}, \mathrm{TIPS}-\mathrm{CH}), 1.34(\mathrm{~d}, J=7.8 \mathrm{~Hz}, 18 \mathrm{H}$, TIPS- $\mathrm{CH}_{3}$ ).

${ }^{13} \mathrm{C}$ NMR (101 MHz, Chloroform-d) $\delta 155.6,137.1,129.4,128.7,127.8,126.9,126.7,95.9$, 18.3, 13.1 .

IR 4319 (w), 4056 (w), 2945 (m), 2867 (m), 1638 (w), 1464 (m), 1327 (s), 1026 (s), 883 (s).

HRMS (ESI) calcd for $\mathrm{C}_{19} \mathrm{H}_{31} \mathrm{OSi}^{+}[\mathrm{M}+\mathrm{H}]^{+} 303.2139$; found 303.2140.

\section{(Z)-Triisopropyl((1-phenylprop-1-en-1-yl)oxy)silane (53).}

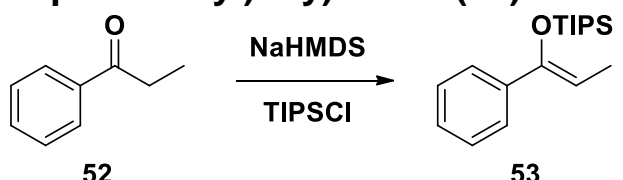

Propiophenone (52) $\left(0.50 \mathrm{~mL}, 3.7 \mathrm{mmol}, 1\right.$ equiv) was solubilized in THF $(15 \mathrm{~mL})$ at $-78{ }^{\circ} \mathrm{C}$ and NaHMDS (2 M solution in THF, $2.1 \mathrm{~mL}, 4.1 \mathrm{mmol}, 1.1$ equiv) was added dropwise. The mixture was stirred 1 hour at room temperature and cooled down to $-78{ }^{\circ} \mathrm{C}$. TIPS-Cl $(0.86 \mathrm{~mL}$, $4.1 \mathrm{mmol}, 1.1$ equiv) was added dropwise and the reaction mixture was stirred at room temperature for $8 \mathrm{~h}$. The solvent was evaporated and the crude product was purified by column chromatography, eluting with pentane and $1 \% \mathrm{NEt}_{3}$ to obtain the pure enol-ether $\mathbf{5 3}(0.46 \mathrm{~g}$, $1.6 \mathrm{mmol}, 43 \%$ yield) as a slightly yellow oil.

${ }^{1} \mathbf{H}$ NMR $(400 \mathrm{MHz}$, Chloroform- $d) \delta 7.50-7.38(\mathrm{~m}, 2 \mathrm{H}, \mathrm{Ar}-\mathrm{H}), 7.33-7.18(\mathrm{~m}, 3 \mathrm{H}, \mathrm{Ar}-\mathrm{H}), 5.06$ $(\mathrm{q}, J=6.8 \mathrm{~Hz}, 1 \mathrm{H}, \mathrm{C}=\mathrm{C}), 1.77\left(\mathrm{~d}, J=6.9 \mathrm{~Hz}, 3 \mathrm{H}, \mathrm{CH}_{3}\right), 1.15-0.97(\mathrm{~m}, 21 \mathrm{H}, \mathrm{TIPS})$.

${ }^{1} \mathrm{H}$ NMR values correspond to the literature. ${ }^{1}$ 


\section{Scope of the reaction [3+2] reaction.}

\subsection{From thymine cyclopropanes.}

\section{General procedures for annulation reaction}
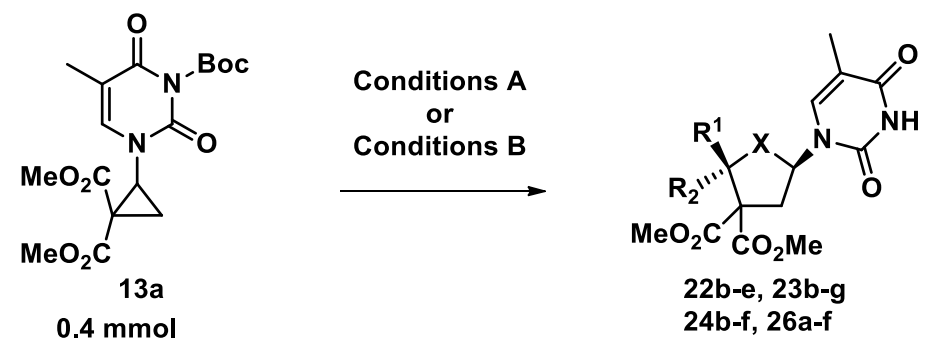

\section{a) Conditions $\mathrm{A}$}

Dimethyl 2-(3-(tert-butoxycarbonyl)-5-methyl-2,4-dioxo-3,4-dihydropyrimidin-1(2H)yl)cyclopropane-1,1-dicarboxylate (13a) $(0.15 \mathrm{~g}, 0.40 \mathrm{mmol} 1.0$ equiv), aldehyde $(0.48 \mathrm{mmol}$, 1.2 equiv) and $\ln (\mathrm{OTf})_{3}(0.045 \mathrm{~g}, 0.080 \mathrm{mmol}, 0.2$ equiv) were stirred under nitrogen in a flamedried sealed microwave vial with anhydrous DCM $(2.0 \mathrm{~mL})$ at room temperature for $2 \mathrm{~h}$. Then, $\mathrm{NEt}_{3}(0.9 \mathrm{~mL})$ was added to quench the reaction and the crude mixture was concentrated under reduced pressure. After a rapid filtration on a silica plug with AcOEt and removal of the solvent, the crude product was heated at $70^{\circ} \mathrm{C}$ in EtOH $(3 \mathrm{~mL})$ in a sealed microwave vial for $18 \mathrm{~h}$. The mixture was concentrated under reduced pressure and purified by column chromatography with a gradient mixture of pentane/AcOEt from 7:3 up to 1:1 and the column was washed with straight AcOEt.

\section{b) Conditions B}

Dimethyl 2-(3-(tert-butoxycarbonyl)-5-methyl-2,4-dioxo-3,4-dihydropyrimidin-1(2H)yl)cyclopropane-1,1-dicarboxylate (13a) $(0.15 \mathrm{~g}, 0.40 \mathrm{mmol} 1.0$ equiv) and the ketone or sillylenol ether $(0.48 \mathrm{mmol}, 1.2$ equiv) were stirred under nitrogen in a flame-dried sealed microwave vial with anhydrous DCM $(2.0 \mathrm{~mL})$ and cooled down to $-20{ }^{\circ} \mathrm{C}$. Then, a $0.43 \mathrm{M}$ tin(IV) chloride solution $(0.09 \mathrm{~mL}, 0.04 \mathrm{mmol}, 0.1$ equiv) was added and the reaction mixture was stirred for $2 \mathrm{~h}$ at $-20^{\circ} \mathrm{C}$. $\mathrm{NEt}_{3}(0.9 \mathrm{~mL})$ was added at $-20^{\circ} \mathrm{C}$ to quench the reaction and the reaction mixture was allowed to reach room temperature. The crude mixture was concentrated under reduced pressure. After a rapid filtration on a silica plug with AcOEt and removal of the solvent, the crude product was heated at $70^{\circ} \mathrm{C}$ in $\mathrm{EtOH}(3 \mathrm{~mL})$ in a sealed microwave vial for $18 \mathrm{~h}$. The mixture was concentrated under reduced pressure and purified by column chromatography with a gradient mixture of pentane/AcOEt from 7:3 up to $1: 1$ and the column was washed with straight AcOEt.

\section{Dimethyl-5-(5-methyl-2,4-dioxo-3,4-dihydropyrimidin-1(2H)-yl)-2-phenyldihydrofuran-} $3,3(2 H)$-dicarboxylate $(22 b)$.<smiles>CC(=O)OC(C)(C)C1(C(C)=O)CC1n1cc(C)c(=O)n(C(C)(C)C)c1=O</smiles>

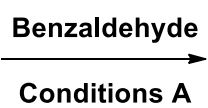<smiles>[CH][PH2+](c1ccccc1)[C@@H]1O[C@H](n2cc(C)c(=O)[nH]c2=O)CC1(C(C)=O)C(C)(C)OC</smiles>

Following the conditions $A$, using benzaldehyde (19) $(0.051 \mathrm{~g}, 0.48 \mathrm{mmol}, 1.2$ equiv), the pure product $22 \mathrm{~b}(0.14 \mathrm{~g}, 0.35 \mathrm{mmol}, 87 \%$ yield $)$ was obtained as a white foamy solid.

$\mathbf{R F}($ pent/AcOEt $(1: 1))=0.64$. 
MP 206.9-208. $1^{\circ} \mathrm{C}$.

${ }^{1} \mathrm{H}$ NMR $(400 \mathrm{MHz}$, Chloroform- $d) \delta 8.47(\mathrm{~s}, 1 \mathrm{H}$, thymine $\mathrm{NH}), 7.94(\mathrm{~d}, J=1.6 \mathrm{~Hz}, 1 \mathrm{H}$, thymine vinyl-CH), $7.48-7.40(\mathrm{~m}, 2 \mathrm{H}, \mathrm{Ar}-\mathrm{H}), 7.40-7.27(\mathrm{~m}, 3 \mathrm{H}, \mathrm{Ar}-\mathrm{H}), 6.37(\mathrm{t}, J=7.6 \mathrm{~Hz}, 1 \mathrm{H}$, tetrahydrofuran- $\mathrm{NCH}), 5.61(\mathrm{~s}, 1 \mathrm{H}$, tetrahydrofuran- $\mathrm{CH}), 3.81(\mathrm{~s}, 3 \mathrm{H}$, ester methyl), $3.13(\mathrm{~s}$, $3 \mathrm{H}$, ester methyl), 2.95 (dd, $J=14.5,7.6 \mathrm{~Hz}, 1 \mathrm{H}$, tetrahydrofuran- $\left.\mathrm{CH}_{2}\right), 2.78$ (dd, $J=14.5,7.7$ $\mathrm{Hz}, 1 \mathrm{H}$, tetrahydrofuran- $\left.\mathrm{CH}_{2}\right), 2.06(\mathrm{~d}, J=1.3 \mathrm{~Hz}, 3 \mathrm{H}$, thymine methyl).

${ }^{13} \mathrm{C}$ NMR (101 MHz, Chloroform- $d$ ) $\delta 169.9,169.8,163.3,150.4,135.9,135.3,128.7,128.1$, 126.5, 112.1, 82.4, 81.4, 63.8, 53.1, 52.7, 39.0, 12.8.

IR 3192 (w), 3069 (w), 1729 (s), 1695 (s), 1512 (m), 1281 (s), 1225 (m), 1094 (m), 1054 (m), $915(\mathrm{~m})$.

HRMS (ESI) calcd for $\mathrm{C}_{19} \mathrm{H}_{20} \mathrm{~N}_{2} \mathrm{NaO}_{7}{ }^{+}[\mathrm{M}+\mathrm{Na}]^{+}$411.1163; found 411.1168.

Dimethyl-2-(4-fluorophenyl)-5-(5-methyl-2,4-dioxo-3,4-dihydropyrimidin-1(2H)yl)dihydrofuran-3,3(2H)-dicarboxylate (22c).<smiles>CC(=O)C1(C(C)=O)CC1n1cc(C)c(=O)n(C(C)(C)C)c1=O</smiles>
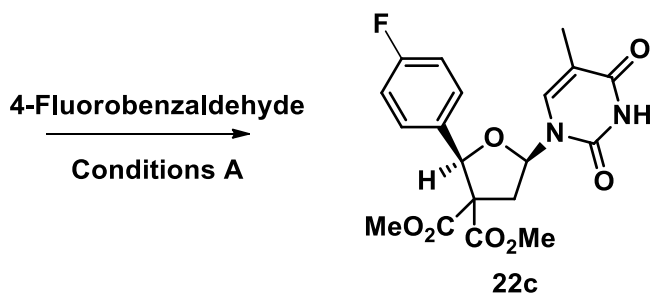

Following the conditions $\mathrm{A}$, using 4-fluorobenzaldehyde $(0.060 \mathrm{~g}, 0.48 \mathrm{mmol}, 1.2$ equiv), the pure product $22 \mathrm{c}(0.13 \mathrm{~g}, 0.32 \mathrm{mmol}, 79 \%$ yield $)$ was obtained as a slightly yellow solid.

$\mathbf{R F}($ pent/AcOET $(1: 1))=0.63$.

MP 218.8-220.3 ${ }^{\circ} \mathrm{C}$.

${ }^{1} \mathrm{H}$ NMR $(400 \mathrm{MHz}$, Chloroform- $d) \delta 8.54-8.46(\mathrm{~m}, 1 \mathrm{H}$, thymine $\mathrm{NH}), 7.93(\mathrm{~d}, J=1.5 \mathrm{~Hz}, 1 \mathrm{H}$, thymine vinyl-CH), $7.49-7.39(\mathrm{~m}, 2 \mathrm{H}, \mathrm{Ar}-\mathrm{H}), 7.10-6.99(\mathrm{~m}, 2 \mathrm{H}, \mathrm{Ar}-\mathrm{H}), 6.37(\mathrm{t}, J=7.6 \mathrm{~Hz}$, $1 \mathrm{H}$, tetrahydrofuran- $\mathrm{NCH}), 5.56(\mathrm{~s}, 1 \mathrm{H}$, tetrahydrofuran- $\mathrm{CH}), 3.81(\mathrm{~s}, 3 \mathrm{H}$, ester methyl), 3.20 (s, $3 \mathrm{H}$, ester methyl), $2.93\left(\mathrm{dd}, J=14.6,7.5 \mathrm{~Hz}, 1 \mathrm{H}\right.$, tetrahydrofuran- $\mathrm{CH}_{2}$ ), 2.77 (dd, $J=14.6$, $7.8 \mathrm{~Hz}, 1 \mathrm{H}$, tetrahydrofuran- $\left.\mathrm{CH}_{2}\right), 2.05(\mathrm{~d}, J=1.2 \mathrm{~Hz}, 3 \mathrm{H}$, thymine methyl).

${ }^{13} \mathrm{C}$ NMR (101 MHz, Chloroform- $\left.d\right) \delta 169.8,162.9(\mathrm{~d}, J=249 \mathrm{~Hz}), 150.4,135.8,131.0$ (d, $J=$ $3.2 \mathrm{~Hz}$ ), $128.5(\mathrm{~d}, J=8.2 \mathrm{~Hz}), 128.4,115.2(\mathrm{~d}, J=21.6 \mathrm{~Hz}), 115,0,112.2,81.7,81.2,63.6$, $53.2,52.9,38.8,12.8$.

IR 3192 (w), 3069 (w), 1729 (s), 1695 (s), 1512 (m), 1281 (s), 1225 (m), 1094 (m), 1054 (m), $915(\mathrm{~m})$.

HRMS (ESI) calcd for $\mathrm{C}_{19} \mathrm{H}_{19} \mathrm{FN}_{2} \mathrm{NaO}_{7}^{+}[\mathrm{M}+\mathrm{Na}]^{+}$429.1068; found 429.1055.

The crystal structure has been deposited at the Cambridge Crystallographic Data Centre and allocated the deposition number: CCDC 995573. 


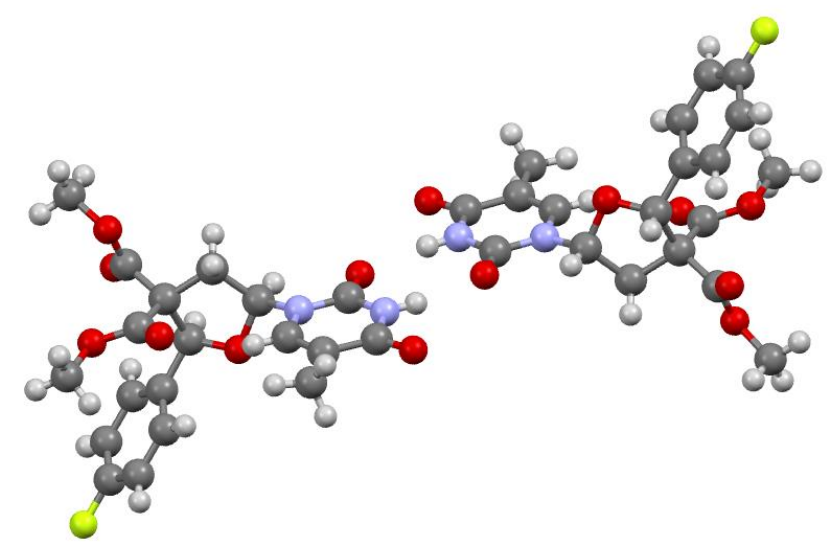

\section{Dimethyl-5-(5-methyl-2,4-dioxo-3,4-dihydropyrimidin-1(2H)-yl)-2-((E)- styryl)dihydrofuran-3,3(2H)-dicarboxylate. (22d)}<smiles>COC(=O)C1(C(C)(C)OC(C)=O)CC1n1cc(C)c(=O)n(C(C)(C)C)c1=O</smiles><smiles>CC(C)(C)[C@H]1CCCCCO1</smiles><smiles>COC(=O)C1(C(C)=O)C[C@H](n2cc(C)c(=O)[nH]c2=O)O[C@@H]1/C=C/c1ccccc1</smiles>

Following the conditions $A$, using cinnamaldehyde $(0.063 \mathrm{~g}, 0.48 \mathrm{mmol}, 1.2$ equiv), a mixture of diastereoisomers (5:1 by integration of methyl esters at $3.76 \mathrm{ppm}$ and $3.71 \mathrm{ppm}) \mathbf{2 2 d}(0.16$ $\mathrm{g}, 0.28 \mathrm{mmol}, 96 \%$ yield) was obtained as a colorless foam.

RF $($ pent/AcOET $(1: 1))=0.67$.

${ }^{1} \mathbf{H}$ NMR $(400 \mathrm{MHz}$, Chloroform- $d) \delta 8.82(\mathrm{~s}, 1 \mathrm{H}, \mathrm{NH}), 7.70(\mathrm{~d}, J=1.3 \mathrm{~Hz}, 1 \mathrm{H}$, thymine vinyl$\mathrm{CH}$, major diastereoisomer), $7.35-7.17(\mathrm{~m}, 10 \mathrm{H}, \mathrm{Ar}-\mathrm{H}$, both diastereoisomers), $7.14 \mathrm{(d}, \mathrm{J}=$ $1.3 \mathrm{~Hz}, 1 \mathrm{H}$, thymine vinyl- $\mathrm{CH}$, minor diastereoisomer), 6.72 (dd, $J=16.0,1.3 \mathrm{~Hz}, 1 \mathrm{H}$ vinyl C$\mathrm{H}$, major and minor diastereoisomers), $6.20(\mathrm{t}, \mathrm{J}=7.3 \mathrm{~Hz}, 1 \mathrm{H}$, tetrahydrofuran- $\mathrm{NCH}$, major diastereoisomer), 6.16-6.05 $(\mathrm{m}, 2 \mathrm{H}$, vinyl $\mathrm{C}-\mathrm{H}$ and tetrahydrofuran- $\mathrm{NCH}$, minor diastereoisomer), 6.11 (dd, $J=16.0,6.2 \mathrm{~Hz}, 1 \mathrm{H}$, vinyl $\mathrm{C}-\mathrm{H}$, major diastereoisomer), 5.49 (dd, $J$ $=6.2,1.4 \mathrm{~Hz}, 1 \mathrm{H}$, tetrahydrofuran- $\mathrm{CH}$, minor diastereoisomer), 5.04 (dd, $J=6.3,1.4 \mathrm{~Hz}, 1 \mathrm{H}$, tetrahydrofuran- $\mathrm{CH}_{2}$, major diastereoisomer), $3.76 \mathrm{(s}, 3 \mathrm{H}$, ester methyl, major diastereoisomer), 3.71 (s, 3H, ester methyl, minor diastereoisomer), 3.59 (s, 6H, ester methyl, minor and major diastereoisomers), $3.24\left(\mathrm{dd}, J=14.3,6.8 \mathrm{~Hz}, 1 \mathrm{H}\right.$, tetrahydrofuran- $\mathrm{CH}_{2}$, minor diastereoisomer), $2.82-2.69\left(\mathrm{~m}, 2 \mathrm{H}\right.$, tetrahydrofuran $-\mathrm{CH}_{2}$, major diastereoisomer), 2.58 (dd, $J=14.3,5.4 \mathrm{~Hz}, 1 \mathrm{H}$, tetrahydrofuran- $\mathrm{CH}_{2}$, minor diastereoisomer), $1.93(\mathrm{~d}, J=1.3 \mathrm{~Hz}, 3 \mathrm{H}$, thymine methyl, major diastereoisomer), $1.88(\mathrm{~d}, J=1.2 \mathrm{~Hz}, 3 \mathrm{H}$, thymine methyl minor diastereoisomer).

${ }^{13} \mathrm{C}$ NMR (101 MHz, Chloroform-d) $\delta 169.6,169.5,169.1,169.1,168.1,163.6,150.5,150.1$, 135.9, 133.7, 133.2, 128.7, 128.4, 128.3, 126.7, 126.7, 123.1, 122.3, 111.7, 110.7, 83.8, 82.2, $81.5,64.1,62.8,53.3,53.2,53.1,39.7,38.9,12.8,12.6$.

One carbon of the major diastereoisomer in the aromatic region is unresolved.

Six carbons of the minor diastereoisomer are unresolved.

IR 3201 (w), 3073 (w), 2953 (w), 1731 (s), 1688 (s), 1468 (m), 1435 (m), 1284 (s), 1085 (s), $972(\mathrm{~m}), 913(\mathrm{~m}), 734(\mathrm{~s})$. 
HRMS (ESI) calcd for $\mathrm{C}_{21} \mathrm{H}_{23} \mathrm{~N}_{2} \mathrm{O}_{7}^{+}[\mathrm{M}+\mathrm{H}]^{+}$415.1500; found 415.1502.

Dimethyl

2-isopropyl-5-(5-methyl-2,4-dioxo-3,4-dihydropyrimidin-1(2H)yl)dihydrofuran-3,3(2H)-dicarboxylate (22e).<smiles>CC(=O)OC(C)(C)C1CC1n1cc(C)c(=O)n(C(C)(C)C)c1=O</smiles>

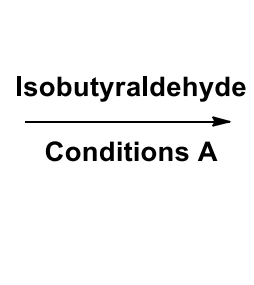<smiles>CC(=O)[C@H]1CC(n2cc(C)c(=O)[nH]c2=O)[C@H](C(C)C)[C@@H](C(C)C)[C@H]1C(C)C</smiles>

Following the conditions $A$, using isobutyraldehyde $(0.035 \mathrm{~g}, 0.48 \mathrm{mmol}, 1.2$ equiv), the pure product $22 \mathrm{e}(0.11 \mathrm{~g}, 0.30 \mathrm{mmol}, 75 \%$ yield $)$ was obtained as a colorless foam.

$\mathbf{R F}($ AcOEt/pent $(1: 1))=0.42$.

MP $157.7-160.8^{\circ} \mathrm{C}$.

${ }^{1} \mathrm{H}$ NMR $(400 \mathrm{MHz}$, Chloroform- $d) \delta 9.45(\mathrm{~s}, 1 \mathrm{H}, \mathrm{N}-\mathrm{H}), 7.62(\mathrm{~d}, J=1.5 \mathrm{~Hz}, 1 \mathrm{H}$, thymine vinyl$\mathrm{CH}), 6.12(\mathrm{t}, J=7.1 \mathrm{~Hz}, 1 \mathrm{H}$, tetrahydrofuran- $\mathrm{NCH}), 4.14(\mathrm{~d}, J=7.8 \mathrm{~Hz}, 1 \mathrm{H}$, tetrahydrofuran$\mathrm{OCH}$ ), $3.80(\mathrm{~s}, 3 \mathrm{H}$, ester methyl), $3.78(\mathrm{~s}, 3 \mathrm{H}$, ester methyl), $2.70(\mathrm{dd}, J=14.5,6.5 \mathrm{~Hz}, 1 \mathrm{H}$, tetrahydrofuran- $\left.\mathrm{CH}_{2}\right), 2.63\left(\mathrm{dd}, J=14.5,7.6 \mathrm{~Hz}, 1 \mathrm{H}\right.$, tetrahydrofuran- $\left.\mathrm{CH}_{2}\right), 1.98(\mathrm{~d}, J=1.3 \mathrm{~Hz}$, $3 \mathrm{H}$, thymine methyl), 1.92 (dt, $J=13.7,7.0 \mathrm{~Hz}, 1 \mathrm{H}$, iso-propyl $\mathrm{C}-\mathrm{H}), 1.03(\mathrm{t}, J=6.8 \mathrm{~Hz}, 6 \mathrm{H}$, iso-propyl $\mathrm{CH}_{3}$ ).

${ }^{13} \mathrm{C}$ NMR $(101 \mathrm{MHz}$, Chloroform- $d) \delta 170.5,170.1,163.9,150.7,135.9,111.1,87.2,81.4,60.6$, 53.0, 41.3, 30.0, 20.1, 19.5, 12.7.

IR 3194 (w), 2960 (w), 2929 (w), 1730 (s), 1683 (s), 1468 (m), 1436 (m), 1275 (s), 1237 (m), $1205(\mathrm{~m}), 1081(\mathrm{~m}), 916(\mathrm{~m}), 733(\mathrm{~s})$.

HRMS (ESI) calcd for $\mathrm{C}_{16} \mathrm{H}_{23} \mathrm{~N}_{2} \mathrm{O}_{7}^{+}[\mathrm{M}+\mathrm{H}]^{+} 355.1500$; found 355.1502.

Dimethyl-2-methyl-5-(5-methyl-2,4-dioxo-3,4-dihydropyrimidin-1(2H)-yl)-2phenyldihydrofuran-3,3(2H)-dicarboxylate (23b).<smiles>CC(=O)C1([O-])CC1n1cc(C)c(=O)n(C(C)(C)C)c1=O</smiles>

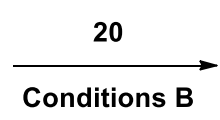

$12 a$<smiles>CC(=O)C1(C(C)=O)[C@@H](n2cc(C)c(=O)[nH]c2=O)O[C@@](C)(c2ccccc2)C1(C)C(C)(C)C</smiles>

Following the conditions $B$, using acetophenone (20) $(0.058 \mathrm{~g}, 0.48 \mathrm{mmol}, 1.2$ equiv), the pure product 23b $(0.15 \mathrm{~g}, 0.38 \mathrm{mmol}, 94 \%$ yield) was obtained as a white solid.

$\mathbf{R F}($ AcOEt/pent $(1: 1))=0.36$.

MP $246.8-247.3^{\circ} \mathrm{C}$.

${ }^{1} \mathrm{H}$ NMR $(400 \mathrm{MHz}$, Chloroform- $d) \delta 8.59(\mathrm{~s}, 1 \mathrm{H}$, thymine $\mathrm{NH}), 8.18(\mathrm{~d}, J=1.7 \mathrm{~Hz}, 1 \mathrm{H}$, thymine vinyl-CH), $7.72-7.65(\mathrm{~m}, 2 \mathrm{H}, \mathrm{Ar}-\mathrm{H}), 7.38-7.24(\mathrm{~m}, 3 \mathrm{H}, \mathrm{Ar}-\mathrm{H}), 6.43(\mathrm{dd}, J=8.2,5.6 \mathrm{~Hz}, 1 \mathrm{H}$, tetrahydrofuran- $\mathrm{NCH}$ ), $3.79(\mathrm{~s}, 3 \mathrm{H}$, ester methyl), $3.16(\mathrm{~s}, 3 \mathrm{H}$, ester methyl), $3.11(\mathrm{dd}, J=15.0$, 
$8.2 \mathrm{~Hz}, 1 \mathrm{H}$, tetrahydrofuran- $\left.\mathrm{CH}_{2}\right) 2.77\left(\mathrm{dd}, J=14.9,5.5 \mathrm{~Hz}, 1 \mathrm{H}\right.$, tetrahydrofuran- $\left.\mathrm{CH}_{2}\right) 2.04(\mathrm{~d}$, $J=1.3 \mathrm{~Hz}, 3 \mathrm{H}$, thymine methyl), $1.86(\mathrm{~s}, 3 \mathrm{H}$, tetrahydrofuran methyl).

${ }^{13} \mathrm{C}$ NMR (101 MHz, Chloroform- $d$ ) $\delta 170.4,168.8,163.6,150.7,140.5,136.9,127.9,127.8$, $125.8,110.9,88.0,80.7,67.1,52.8,52.6,39.6,25.7,12.8$.

IR $1733(\mathrm{~m}), 1706(\mathrm{~m}), 1663(\mathrm{~m}), 1272(\mathrm{~s}), 1207(\mathrm{w}), 1126(\mathrm{w}), 1077(\mathrm{w})$.

HRMS (ESI) calcd for $\mathrm{C}_{20} \mathrm{H}_{22} \mathrm{NNaO}_{7}^{+}[\mathrm{M}+\mathrm{Na}]^{+}$425.1319; found 425.1320.

Dimethyl-2-(4-fluorophenyl)-2-methyl-5-(5-methyl-2,4-dioxo-3,4-dihydropyrimidin$1(2 \mathrm{H})$-yl)dihydrofuran-3,3(2H)-dicarboxylate (23c).
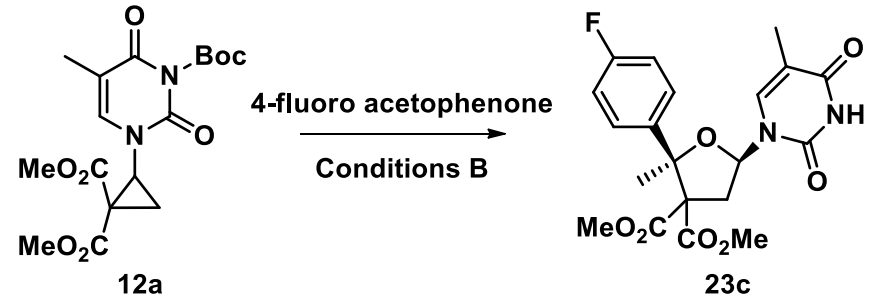

Following the conditions $B$, using 4-fluoroacetophenone $(0.067 \mathrm{~g}, 0.48 \mathrm{mmol}, 1.2$ equiv), the pure product $23 \mathrm{c}(0.16 \mathrm{~g}, 0.37 \mathrm{mmol}, 93 \%$ yield $)$ was obtained as a white solid.

$\mathbf{R F}($ AcOEt/pent $(1: 1))=0.40$.

MP 248.8-250.2 ${ }^{\circ} \mathrm{C}$.

${ }^{1} \mathrm{H}$ NMR $\left(400 \mathrm{MHz}, \mathrm{DMSO}-\mathrm{d}_{6}\right) \delta 11.47(\mathrm{~s}, 1 \mathrm{H}$, thymine $\mathrm{NH}), 8.07(\mathrm{~d}, J=1.5 \mathrm{~Hz}, 1 \mathrm{H}$, thymine vinyl-CH), $7.70-7.60(\mathrm{~m}, 2 \mathrm{H}, \mathrm{Ar}-\mathrm{H}), 7.20(\mathrm{t}, J=8.9 \mathrm{~Hz}, 2 \mathrm{H}, \mathrm{Ar}-\mathrm{H}), 6.36(\mathrm{dd}, J=8.3,5.5 \mathrm{~Hz}$, $1 \mathrm{H}$, tetrahydrofuran- $\mathrm{NCH}), 3.75(\mathrm{~s}, 3 \mathrm{H}$, ester methyl), $3.26(\mathrm{dd}, J=14.9,8.3 \mathrm{~Hz}, 1 \mathrm{H}$, , tetrahydrofuran- $\left.\mathrm{CH}_{2}\right), 3.13(\mathrm{~s}, 3 \mathrm{H}$, ester methyl), $2.71(\mathrm{dd}, J=14.9,5.6 \mathrm{~Hz}, 1 \mathrm{H}$, , tetrahydrofuran- $\left.\mathrm{CH}_{2}\right), 1.87(\mathrm{~d}, J=1.2 \mathrm{~Hz}, 3 \mathrm{H}$, thymine methyl), $1.79(\mathrm{~s}, 3 \mathrm{H}$, tetrahydrofuran methyl).

${ }^{13} \mathrm{C}$ NMR $\left(101 \mathrm{MHz}\right.$, DMSO- $\left.d_{6}\right) \delta 170.6,168.9,164.1,161.9(\mathrm{~d}, J=243.9 \mathrm{~Hz}), 151.1,137.4$ (d, $J=3.0 \mathrm{~Hz}), 136.7,128.4(\mathrm{~d}, J=8.2 \mathrm{~Hz}), 114.9(\mathrm{~d}, J=21.3 \mathrm{~Hz}), 109.9,87.1,80.6,67.2$, 53.3, 53.0, 38.5, 25.9, 13.2 .

IR 3221 (w), 3072 (w), 2948 (w), $1513(\mathrm{~m}), 1439$ (w), 1269 (s), $1128(\mathrm{~m}), 1077(\mathrm{~m}), 964(\mathrm{w})$, $913(\mathrm{~m}), 842(\mathrm{~m})$.

HRMS (ESI) calcd for $\mathrm{C}_{20} \mathrm{FH}_{22} \mathrm{~N}_{2} \mathrm{O}_{7}{ }^{+}[\mathrm{M}+\mathrm{H}]^{+}$421.1406; found 421.1405.

Dimethyl 2-methyl-5-(5-methyl-2,4-dioxo-3,4-dihydropyrimidin-1(2H)-yl)-2-((E)styryl)dihydrofuran-3,3(2H)-dicarboxylate. (23d)
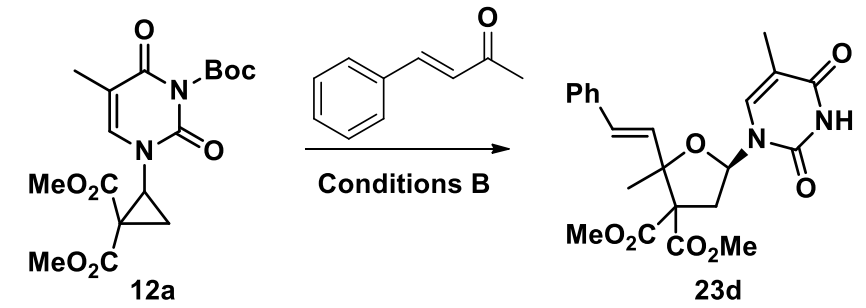

Following the conditions $\mathrm{B}$, using $(E)$-4-phenylbut-3-en-2-one $(0.070 \mathrm{~g}, 0.48 \mathrm{mmol}, 1.2$ equiv), a mixture of unseparable diastereoisomers (ratio 2:1 obtained by integration of methyl esters at $3.64 \mathrm{ppm}$ and $3.55 \mathrm{ppm}) \mathbf{2 3 d}(0.14 \mathrm{~g}, 0.34 \mathrm{mmol}, 79 \%$ yield) was obtained as a colorless foam. 
$\mathbf{R F}($ pent/AcOET $(1: 1))=0.4$

${ }^{1} \mathbf{H}$ NMR (400 MHz, Chloroform-d) $\delta 9.19$ (br.s, $\left.1 \mathrm{H}, \mathrm{N}-\mathrm{H}\right), 7.84(\mathrm{~d}, J=1.3 \mathrm{~Hz}, 1 \mathrm{H}$, , thymine vinyl- $\mathrm{CH}$, major diastereoisomer), $7.59(\mathrm{~d}, J=1.3 \mathrm{~Hz}, 1 \mathrm{H}$, thymine vinyl-CH, minor diastereoisomer), $7.36-7.16(\mathrm{~m}, 5 \mathrm{H}, \mathrm{Ar}-\mathrm{H}), 6.69(\mathrm{~d}, J=15.9 \mathrm{~Hz}, 1 \mathrm{H}$, vinyl $\mathrm{C}-\mathrm{H}$, minor diastereomer), $6.65(\mathrm{~d}, J=16.1 \mathrm{~Hz}, 1 \mathrm{H}$, vinyl $\mathrm{C}-\mathrm{H}$, major diastereomer), $6.39(\mathrm{~d}, J=16.1 \mathrm{~Hz}$, $1 \mathrm{H}$, vinyl $\mathrm{C}-\mathrm{H}$, major diastereomer), $6.32(\mathrm{~d}, \mathrm{~J}=16.0 \mathrm{~Hz}, 1 \mathrm{H}$, vinyl $\mathrm{C}-\mathrm{H}$, minor diastereomer), $6.25(\mathrm{~m}, 1 \mathrm{H}$, tetrahydrofuran- $\mathrm{NCH}$, major and minor diastereoisomers $), 3.75(\mathrm{~s}, 3 \mathrm{H}$, ester methyl, major diastereoisomer), $3.72(\mathrm{~s}, 3 \mathrm{H}$, ester methyl, minor diastereoisomer), $3.64(\mathrm{~s}, 3 \mathrm{H}$, ester methyl, minor diastereoisomer), 3.55 (s, 3H, ester methyl, major diastereoisomer), 3.13 (dd, $J=14.7,7.1 \mathrm{~Hz}, 1 \mathrm{H}$, , tetrahydrofuran- $\mathrm{CH}_{2}$, minor diastereoisomer), 3.09 (dd, $J=14.9$, $7.8 \mathrm{~Hz}, 1 \mathrm{H}$, tetrahydrofuran- $\mathrm{CH}_{2}$, major diastereoisomer), 2.57 (dd, $J=14.9,5.5 \mathrm{~Hz}, 1 \mathrm{H}$, tetrahydrofuran- $\mathrm{CH}_{2}$, major diastereoisomer), 2.51 (dd, $J=14.8,5.6 \mathrm{~Hz}, 1 \mathrm{H}$, tetrahydrofuran$\mathrm{CH}_{2}$, minor diastereoisomer), 1.91 (d, $J=1.2 \mathrm{~Hz}, 3 \mathrm{H}$, thymine methyl, minor diastereoisomer), $1.89(\mathrm{~d}, J=1.2 \mathrm{~Hz}, 3 \mathrm{H}$, thymine methyl, major diastereoisomer), 1.61 (s, 3H, tetrahydrofuran methyl, minor diastereoisomer), 1.52 ( $\mathrm{s}, 3 \mathrm{H}$, tetrahydrofuran methyl, major diastereoisomer).

${ }^{13} \mathrm{C}$ NMR (101 MHz, Chloroform- $\left.d\right) \delta 169.9,169.4,168.7,168.5,164.1,164.0,150.8,150.5$, 136.7, 136.2, 136.0, 130.1, 129.8, 128.8, 128.7, 128.7, 128.7, 128.1, 126.7, 126.6, 110.4, 109.8, 87.6, 86.4, 84.5, 82.2, 66.5, 66.4, 53.1, 53.1, 53.0, 39.6, 38.9, 24.9, 24.1, 12.8, 12.7. 3 carbons are unresolved.

IR 3180 (w), 3044 (w), 1736 (s), 1688 (s), 1458 (w), 1258 (s), 1076 (w), 733 (s).

HRMS (ESI) calcd for $\mathrm{C}_{22} \mathrm{H}_{25} \mathrm{~N}_{2} \mathrm{O}_{7}^{+}[\mathrm{M}+\mathrm{H}]^{+}$429.1656; found 429.1660.

\section{Dimethyl-2-methyl-5-(5-methyl-2,4-dioxo-3,4-dihydropyrimidin-1(2H)-yl)-2- phenethyldihydrofuran-3,3(2H)-dicarboxylate (23e).}
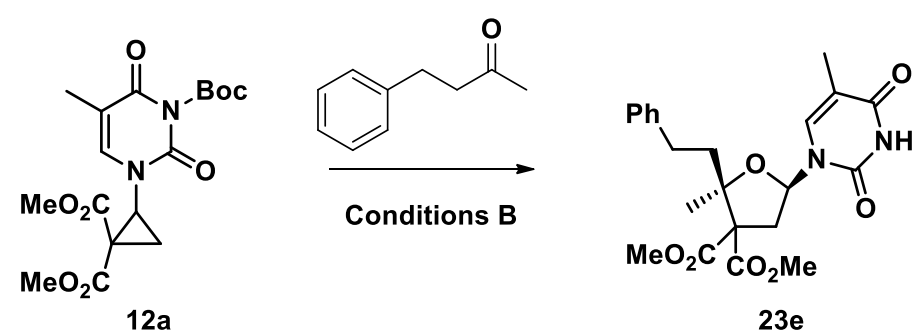

Following the conditions $B$, using 4-phenylbutan-2-one $(0.071 \mathrm{~g}, 0.48 \mathrm{mmol}, 1.2$ equiv), the pure product $23 \mathrm{e}(0.15 \mathrm{~g}, 0.34 \mathrm{mmol}, 85 \%$ yield $)$ was obtained as a slightly yellow solid.

MP $167.9-168.7^{\circ} \mathrm{C}$.

$\mathbf{R F}($ pent/AcOET $(1: 1))=0.19$.

${ }^{1} \mathrm{H}$ NMR $(400 \mathrm{MHz}$, Chloroform- $d) \delta 8.07(\mathrm{~s}, 1 \mathrm{H}$, thymine $\mathrm{NH}), 7.74(\mathrm{q}, J=1.2 \mathrm{~Hz}, 1 \mathrm{H}$, thymine vinyl-CH), 7.30 (dd, $J=8.5,6.6 \mathrm{~Hz}, 2 \mathrm{H}, \mathrm{Ar}-\mathrm{H}), 7.24-7.17$ (m, 3H, Ar-H), 6.18 (dd, $J=7.7,5.3$ $\mathrm{Hz}, 1 \mathrm{H}$, tetrahydrofuran- $\mathrm{NCH}), 3.78(\mathrm{~s}, 3 \mathrm{H}$, ester methyl), $3.72(\mathrm{~s}, 3 \mathrm{H}$, ester methyl), $3.23-$ $3.05\left(\mathrm{~m}, 1 \mathrm{H}\right.$, tetrahydrofuran- $\left.\mathrm{CH}_{2}\right), 2.92-2.69\left(\mathrm{~m}, 2 \mathrm{H}\right.$, benzylic $\left.-\mathrm{CH}_{2}\right), 2.54(\mathrm{dd}, J=15.0,5.4$ $\mathrm{Hz}, 1 \mathrm{H}$, tetrahydrofuran- $\left.\mathrm{CH}_{2}\right), 2.28$ (ddd, $\left.J=13.7,11.7,6.0 \mathrm{~Hz}, 1 \mathrm{H}, \mathrm{CH}_{2}\right), 2.00-1.90(\mathrm{~m}, 4 \mathrm{H}$, thymine methyl and $\left.\mathrm{CH}_{2}\right), 1.47(\mathrm{~s}, 3 \mathrm{H}$, tetrahydrofuran methyl).

${ }^{13}$ C NMR (101 MHz, Chloroform- $\left.d\right) \delta 170.5,168.9,163.5,150.3,141.5,136.6,128.5,128.3$, $126.1,110.2,87.0,82.0,66.2,53.1,52.9,4.5,38.8,30.3,21.9,12.7$. 
IR 3193 (w), 3060 (w), 2957 (w), 1735 (s), 1692 (s), 1468 (m), 1266 (s), 1097 (m), 914 (m).

HRMS (ESI) calcd for $\mathrm{C}_{22} \mathrm{H}_{27} \mathrm{~N}_{2} \mathrm{O}_{7}^{+}[\mathrm{M}+\mathrm{H}]^{+}$431.1813; found 431.1802.

The crystal structure has been deposited at the Cambridge Crystallographic Data Centre and allocated the deposition number: CCDC 994735

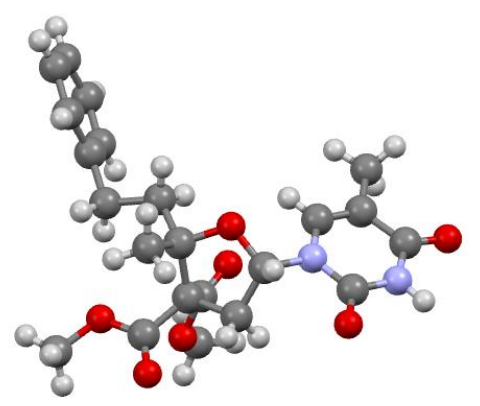

Dimethyl 2-(5-methyl-2,4-dioxo-3,4-dihydropyrimidin-1(2H)-yl)-1-oxaspiro[4.5]decane4,4-dicarboxylate. (23f)<smiles></smiles>

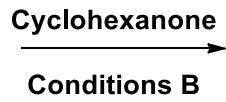<smiles>CC(=O)C1(C(C)=O)O[C@H](n2cc(C)c(=O)[nH]c2=O)CC12CCCCC2</smiles>

23f

Following the conditions $\mathrm{B}$, using cyclohexanone $(0.047 \mathrm{~g}, 0.48 \mathrm{mmol}, 1.2$ equiv), the pure product $23 \mathrm{f}(0.15 \mathrm{~g}, 0.39 \mathrm{mmol}, 97 \%$ yield) was obtained as a colorless foamy solid.

$\mathbf{R F}($ AcOEt/pent $(1: 1))=0.3$.

MP $184.1-185.6^{\circ} \mathrm{C}$.

${ }^{1} \mathrm{H}$ NMR $(400 \mathrm{MHz}$, Chloroform- $d) \delta 8.25-8.08$ (br s, $1 \mathrm{H}$, thymine $\left.\mathrm{NH}\right), 7.70(\mathrm{~d}, J=1.5 \mathrm{~Hz}$, $1 \mathrm{H}$, thymine vinyl- $\mathrm{CH}$ ), 6.03 (dd, $J=7.6,5.2 \mathrm{~Hz}, 1 \mathrm{H}$, tetrahydrofuran- $\mathrm{NCH}), 3.71(\mathrm{~s}, 3 \mathrm{H}$, ester methyl), $3.68\left(\mathrm{~s}, 3 \mathrm{H}\right.$, ester methyl), $3.11\left(\mathrm{dd}, J=14.9,7.6 \mathrm{~Hz}, 1 \mathrm{H}\right.$, tetrahydrofuran- $\left.\mathrm{CH}_{2}\right), 2.39$ (dd, $J=14.9,5.3 \mathrm{~Hz}, 1 \mathrm{H}$, tetrahydrofuran- $\left.\mathrm{CH}_{2}\right), 1.91(\mathrm{~d}, J=1.2 \mathrm{~Hz}, 3 \mathrm{H}$, thymine methyl), 1.75 $-1.47(\mathrm{~m}, 8 \mathrm{H}$, cyclohexane $\mathrm{C}-\mathrm{H}), 1.22-1.12(\mathrm{~m}, 2 \mathrm{H}$, cyclohexane $\mathrm{C}-\mathrm{H})$.

${ }^{13} \mathrm{C}$ NMR (101 MHz, Chloroform-d) $\delta 170.3,168.7,163.6,150.3,136.6,109.9,87.2,82.0,65.8$, $52.9,52.8,39.6,32.4,31.2,25.0,22.7,21.5,12.7$.

IR 3210 (w), 2931 (w), 2856 (w), 1733 (m), 1687 (s), 1437 (w), 1268 (m), 1201 (w), 1095 (m), $911(\mathrm{~m}), 729(\mathrm{~s})$.

HRMS (ESI) calcd for $\mathrm{C}_{18} \mathrm{H}_{24} \mathrm{~N}_{2} \mathrm{NaO}_{7}[\mathrm{M}+\mathrm{Na}]$ 403.1481; found 403.1488.

Dimethyl 2-methyl-5-(5-methyl-2,4-dioxo-3,4-dihydropyrimidin-1(2H)-yl)-2-((E)styryl)dihydrofuran-3,3(2H)-dicarboxylate. $(23 \mathrm{~g})$ 

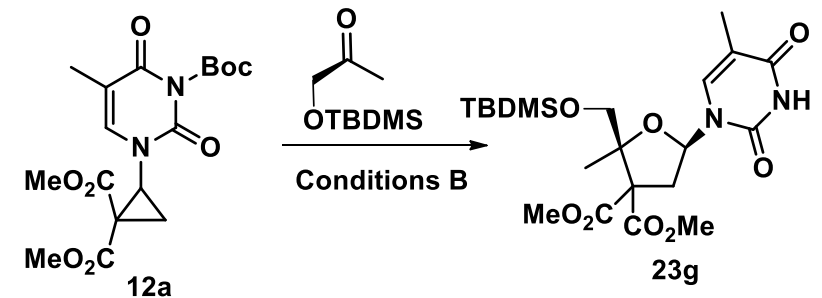

Following the conditions $\mathrm{B}$, using 1-((tert-butyldimethylsilyl)oxy)propan-2-one (0.075 g, 0.48 mmol, 1.2 equiv), a single diasteroisomer $23 \mathrm{~g}(0.072 \mathrm{~g}, 0.15 \mathrm{mmol}, 38 \%$ yield) was obtained as a colorless oil.

$\mathbf{R F}($ pent/AcOET $(1: 1))=0.53$

${ }^{1} \mathrm{H}$ NMR $(400 \mathrm{MHz}$, Chloroform- $d) \delta 8.35(\mathrm{~s}, 1 \mathrm{H}, \mathrm{N}-\mathrm{H}), 7.61(\mathrm{~d}, J=1.3 \mathrm{~Hz}, 1 \mathrm{H}$, thymine vinyl$\mathrm{CH}), 6.25(\mathrm{dd}, J=8.0,5.8 \mathrm{~Hz}, 1 \mathrm{H}$, tetrahydrofuran- $\mathrm{NCH}), 4.09\left(\mathrm{~d}, J=10.9 \mathrm{~Hz}, 1 \mathrm{H}, \mathrm{CH}_{2} \mathrm{O}\right)$, 3.80 (s, $3 \mathrm{H}$, ester methyl), 3.72 (s, 3H, ester methyl), 3.68 (d, $\mathrm{J}=10.9 \mathrm{~Hz}, 1 \mathrm{H}, \mathrm{CH}_{2} \mathrm{O}$ ), 3.05 (dd, $J=13.3,5.8 \mathrm{~Hz}, 1 \mathrm{H}$, tetrahydrofuran- $\left.\mathrm{CH}_{2}\right), 2.72(\mathrm{dd}, J=13.3,8.1 \mathrm{~Hz}, 1 \mathrm{H}$, tetrahydrofuran$\mathrm{CH}_{2}$ ), $1.92(\mathrm{~d}, J=1.1 \mathrm{~Hz}, 3 \mathrm{H}$, thymine methyl), 1.31 (s, 3H, tetrahydrofuran methyl), 0.82 (s, 9H, TBDMS), 0.05 (s, 6H, TBDMS).

${ }^{13} \mathrm{C} \mathrm{NMR}\left(101 \mathrm{MHz}, \mathrm{CDCl}_{3}\right) \delta 170.1,168.2,163.7,150.1,135.9,109.3,88.0,85.6,69.1,64.2$, $53.1,52.6,40.9,25.6,21.1,18.0,12.8,-5.7,-6.0$.

IR 3180 (w), 3126 (w), 2909 (w), 1684 (s), 1443 (s), 1096 (w), 848 (s).

HRMS (ESI) calcd for $\mathrm{C}_{21} \mathrm{H}_{34} \mathrm{~N}_{2} \mathrm{NaO}_{8} \mathrm{Si}^{+}[\mathrm{M}+\mathrm{Na}]^{+}$493.1977; found 493.1982.

\section{Dimethyl-4-(5-methyl-2,4-dioxo-3,4-dihydropyrimidin-1(2H)-yl)-2-phenyl-2- ((triisopropylsilyl)oxy)cyclopentane-1,1-dicarboxylate. (24b)}<smiles>CC(=O)OC(C)(C)C1(C(C)=O)CC1n1cc(C)c(=O)n(C(C)(C)C)c1=O</smiles>
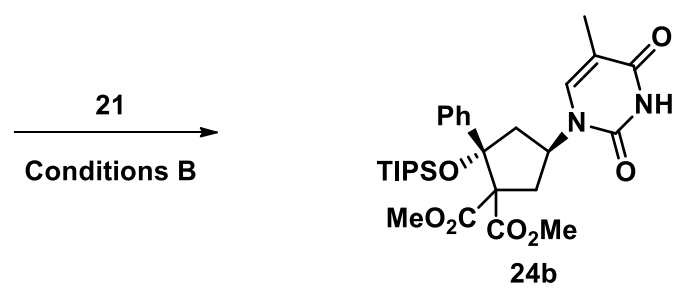

Following the conditions B, using TIPS protected acetophenone (21) $(0.17 \mathrm{~g}, 0.60 \mathrm{mmol}, 1.5$ equiv), the pure product $24 \mathrm{~b}(0.19 \mathrm{~g}, 0.33 \mathrm{mmol}, 84 \%$ yield) was obtained as a white crystalline solid.

$\mathbf{R F}($ pent/AcOEt $(1: 1))=0.56$.

MP $81.8-83.2^{\circ} \mathrm{C}$

${ }^{1} \mathrm{H}$ NMR $(400 \mathrm{MHz}$, Chloroform- $d) \delta 8.40(\mathrm{~s}, 1 \mathrm{H}$, thymine $\mathrm{N}-\mathrm{H}), 7.86(\mathrm{~d}, J=1.6 \mathrm{~Hz}, 1 \mathrm{H}$, thymine vinyl-CH), $7.82-7.74(\mathrm{~m}, 2 \mathrm{H}, \mathrm{Ar}-\mathrm{H}), 7.34-7.27(\mathrm{~m}, 3 \mathrm{H}, \mathrm{Ar}-\mathrm{H}), 5.70$ (ddd, $J=11.4,9.3,5.7$ $\mathrm{Hz}, 1 \mathrm{H}$, cyclopentane- $\mathrm{NCH}), 3.76(\mathrm{~s}, 3 \mathrm{H}$, ester methyl), $3.29(\mathrm{~m}, 4 \mathrm{H}$, ester methyl and cyclopentane- $\mathrm{CH}_{2}$ ), $3.18\left(\mathrm{t}, J=12.4 \mathrm{~Hz}, 1 \mathrm{H}\right.$, cyclopentane- $\mathrm{CH}_{2}$ ), $2.52(\mathrm{dd}, J=12.8,6.8 \mathrm{~Hz}$, $1 \mathrm{H}$, cyclopentane- $\left.\mathrm{CH}_{2}\right), 2.36\left(\mathrm{dd}, J=15.0,7.3 \mathrm{~Hz}, 1 \mathrm{H}\right.$, cyclopentane- $\left.\mathrm{CH}_{2}\right), 2.00(\mathrm{~d}, J=1.2 \mathrm{~Hz}$, $3 \mathrm{H}$, thymine methyl), $1.03-0.97(\mathrm{~m}, 11 \mathrm{H}, \mathrm{TIPS}), 0.97-0.90(\mathrm{~m}, 10 \mathrm{H}, \mathrm{TIPS})$.

${ }^{13} \mathrm{C}$ NMR (101 MHz, Chloroform- $\left.d\right) \delta 172.6,168.1,163.5,151.1,139.9,137.3,128.4,128.1$, $127.4,111.5,88.3,70.3,52.7,52.3,51.5,43.4,38.2,18.2,13.8,12.8$.

IR 2950 (w), 2868 (w), 1681 (s), 1467 (m), 1434 (w), 1392 (w), 1259 (s), 1135 (m), 1090 (m). 
HRMS (ESI) calcd for $\mathrm{C}_{29} \mathrm{H}_{42} \mathrm{~N}_{2} \mathrm{NaO}_{7} \mathrm{Si}^{+}[\mathrm{M}+\mathrm{Na}]^{+}$581.2653; found 581.2660.

The crystal structure has been deposited at the Cambridge Crystallographic Data Centre and allocated the deposition number: CCDC 994948

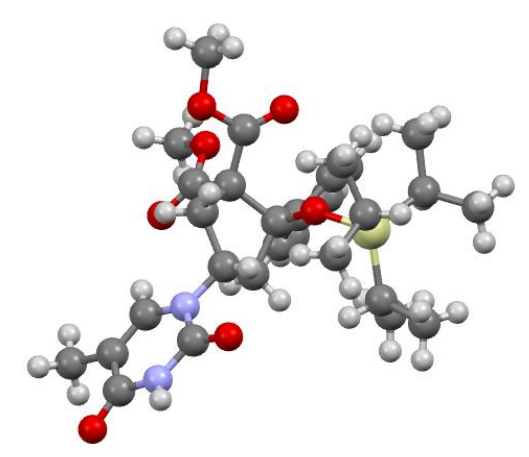

Dibenzyl 4-(5-methyl-2,4-dioxo-3,4-dihydropyrimidin-1(2H)-yl)-2-phenyl-2((triisopropylsilyl)oxy)cyclopentane-1,1-dicarboxylate. (24c)
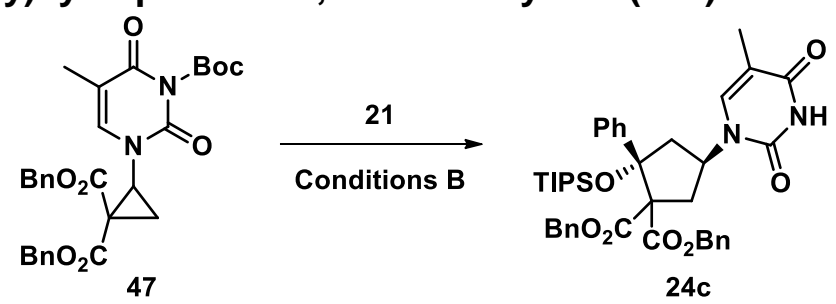

Following the conditions B, using TIPS protected acetophenone $21(0.17 \mathrm{~g}, 0.60 \mathrm{mmol}, 1.5$ equiv) and the corresponding cyclopropane $37(0.21 \mathrm{~g}, 0.40 \mathrm{mmol}, 1$ equiv), the pure product 24c $(0.27 \mathrm{~g}, 0.38 \mathrm{mmol}, 95 \%$ yield $)$ was obtained as a white crystalline solid.

$\mathbf{R F}($ pent/AcOEt $(1: 1))=0.70$.

MP $69.4-73.1^{\circ} \mathrm{C}$.

${ }^{1} \mathrm{H}$ NMR $(400 \mathrm{MHz}$, Chloroform- $d) \delta 8.35(\mathrm{~s}, 1 \mathrm{H}$, thymine $\mathrm{N}-\mathrm{H}), 7.84(\mathrm{~d}, J=1.6 \mathrm{~Hz}, 1 \mathrm{H}$, thymine vinyl-CH), 7.77 (dd, $J=7.3,1.8 \mathrm{~Hz}, 2 \mathrm{H}, \mathrm{Ar}-\mathrm{H}), 7.31-7.11(\mathrm{~m}, 11 \mathrm{H}, \mathrm{Ar}-\mathrm{H}), 6.91-6.84(\mathrm{~m}, 2 \mathrm{H}$, Ar-H), 5.72 (tt, $J=11.5,6.9 \mathrm{~Hz}, 1 \mathrm{H}$, cyclopentane- $\mathrm{NCH}), 5.19-5.03\left(\mathrm{~m}, 2 \mathrm{H}\right.$, benzylic- $\left.\mathrm{CH}_{2}\right)$, $4.76\left(\mathrm{~d}, J=12.3 \mathrm{~Hz}, 1 \mathrm{H}\right.$, benzylic- $\left.\mathrm{CH}_{2}\right), 4.56\left(\mathrm{~d}, J=12.2 \mathrm{~Hz}, 1 \mathrm{H}\right.$, benzylic- $\left.\mathrm{CH}_{2}\right), 3.35(\mathrm{dd}, J=$ $15.1,11.1 \mathrm{~Hz}, 1 \mathrm{H}$, cyclopentane- $\mathrm{CH}_{2}$ ), $3.21\left(\mathrm{t}, J=12.4 \mathrm{~Hz}, 1 \mathrm{H}\right.$, cyclopentane- $\mathrm{CH}_{2}$ ), 2.53 (dd, $J=12.9,6.9 \mathrm{~Hz}, 1 \mathrm{H}$, cyclopentane- $\left.\mathrm{CH}_{2}\right), 2.38\left(\mathrm{dd}, J=15.2,7.0 \mathrm{~Hz}, 1 \mathrm{H}\right.$, cyclopentane- $\left.\mathrm{CH}_{2}\right)$, $1.94(\mathrm{~d}, J=1.2 \mathrm{~Hz}, 3 \mathrm{H}$, thymine methyl), $1.03-0.92(\mathrm{~m}, 21 \mathrm{H}, \mathrm{TIPS})$.

${ }^{13} \mathrm{C}$ NMR $(101 \mathrm{MHz}$, Chloroform-d) $\delta 171.9,167.4,163.4,151.1,139.7,137.3,135.1,134.4$, 128.6, 128.5, 128.4, 128.4, 128.4, 128.3, 128.0, 127.4, 111.5, 88.3, 70.4, 67.7, 67.5, 51.2, 43.6, 38.3, 18.3, 13.9, 12.8.

One carbone not resolved.

IR 3434 (w), 3160 (w), 3035 (w), 2872 (w), 1682 (s), 1456 (w), 1374 (w), 1136 (w), 1025 (m).

HRMS (ESI) calcd for $\mathrm{C}_{41} \mathrm{H}_{50} \mathrm{~N}_{2} \mathrm{NaO}_{7} \mathrm{~S}^{+}[\mathrm{M}+\mathrm{Na}]^{+}$733.3279; found 733.3271.

The crystal structure has been deposited at the Cambridge Crystallographic Data Centre and allocated the deposition number: CCDC 995131. 


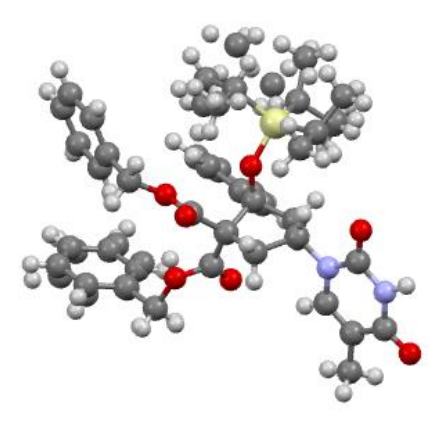

Dimethyl-2-(4-fluorophenyl)-4-(5-methyl-2,4-dioxo-3,4-dihydropyrimidin-1(2H)-yl)-2((triisopropylsilyl)oxy)cyclopentane-1,1-dicarboxylate. (24d)

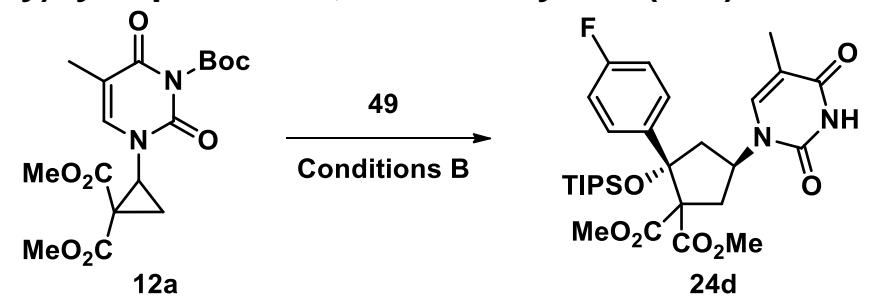

Following the conditions B, using ((1-(4-fluorophenyl)vinyl)oxy)triisopropylsilane (49) $(0.14 \mathrm{~g}$, $0.48 \mathrm{mmol}, 1.2$ equiv), the pure product $24 \mathrm{~d}(0.18 \mathrm{~g}, 0.32 \mathrm{mmol}, 80 \%$ yield) was obtained as a white foamy solid.

MP $105.6-106.7^{\circ} \mathrm{C}$.

$\mathbf{R F}($ pent/AcOET $(1: 1))=0.45$.

${ }^{1} \mathrm{H}$ NMR $(400 \mathrm{MHz}$, Chloroform- $d) \delta 8.14(\mathrm{~d}, J=3.8 \mathrm{~Hz}, 1 \mathrm{H}$, thymine-NH), $7.89-7.78(\mathrm{~m}, 3 \mathrm{H}$, $\mathrm{Ar}-\mathrm{H}$ and thymine vinyl-CH), $7.03(\mathrm{t}, J=8.7 \mathrm{~Hz}, 2 \mathrm{H}, \mathrm{Ar}-\mathrm{H}), 5.71(\mathrm{tt}, J=11.4,7.0 \mathrm{~Hz}, 1 \mathrm{H}$, cyclopentane- $\mathrm{NCH}), 3.79(\mathrm{~s}, 3 \mathrm{H}$, methyl ester), $3.37(\mathrm{~s}, 3 \mathrm{H}$, ester methyl), $3.36-3.31(\mathrm{~m}, 1 \mathrm{H}$, cyclopentane- $\mathrm{CH}_{2}$ ). $3.19\left(\mathrm{t}, J=12.3 \mathrm{~Hz}, 1 \mathrm{H}\right.$, cyclopentane- $\left.\mathrm{CH}_{2}\right), 2.54(\mathrm{dd}, J=12.8,6.8 \mathrm{~Hz}$, $1 \mathrm{H}$, cyclopentane- $\left.\mathrm{CH}_{2}\right), 2.38\left(\mathrm{dd}, J=15.1,7.2 \mathrm{~Hz}, 1 \mathrm{H}\right.$, cyclopentane- $\left.\mathrm{CH}_{2}\right), 2.03(\mathrm{~d}, J=1.2 \mathrm{~Hz}$, $3 \mathrm{H}$, thymine methyl), $1.39-1.23(\mathrm{~m}, 3 \mathrm{H}, \mathrm{TIPS}), 1.09-0.85$ (m, 18H, TIPS).

${ }^{13} \mathrm{C}$ NMR (101 MHz, Chloroform- $d$ ) $\delta 172.6,168.1,162.7$ (d, $\left.J=246.7 \mathrm{~Hz}\right), 163.3,151.0,137.2$, 135.9 (d, $J=3.2 \mathrm{~Hz}), 130.2(\mathrm{~d}, J=8.1 \mathrm{~Hz}), 114.2(\mathrm{~d}, J=21.3 \mathrm{~Hz}), 111.6,87.7,70.2,52.8$, 52.4, 51.4, 43.5, 38.1, 18.2, 13.8, 12.8 .

IR 3196 (w), 2951 (w), 2869 (w), 1681 (s), $1513(w), 1466$ (w), 1260 (m), $1098(\mathrm{~m}), 911(\mathrm{~m})$, $731(s)$.

HRMS (ESI) calcd for $\mathrm{C}_{29} \mathrm{FH}_{42} \mathrm{~N}_{2} \mathrm{O}_{7} \mathrm{Si}^{+}[\mathrm{M}+\mathrm{H}]^{+}$577.2740; found 577.2719.

Dimethyl 4-(5-methyl-2,4-dioxo-3,4-dihydropyrimidin-1(2H)-yl)-2-((E)-styryl)-2((triisopropylsilyl)oxy)cyclopentane-1,1-dicarboxylate. (24e)

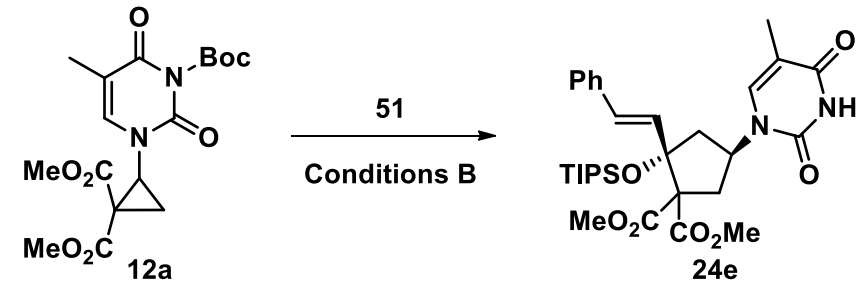


Following the conditions $\mathrm{B}$, using ( $E$ )-triisopropyl((4-phenylbuta-1,3-dien-2-yl)oxy)silane (51) $(0.15 \mathrm{~g}, 0.48 \mathrm{mmol}, 1.2$ equiv), a mixture of unseparable diastereoisomers (ratio 13:1 by integration of the $\mathrm{NC}-\mathrm{H}$ proton at $5.64 \mathrm{ppm}$ and $4.88 \mathrm{ppm}) 24 \mathrm{e}(0.13 \mathrm{~g}, 0.22 \mathrm{mmol}, 55 \%$ yield) was obtained as a colorless foam.

$\mathbf{R F}($ pent/AcOET $(1: 1))=0.54$

${ }^{1} \mathrm{H}$ NMR (400 MHz, Chloroform-d, Major diastereoisomer) $\delta 8.58(\mathrm{~s}, 1 \mathrm{H}, \mathrm{N}-\mathrm{H}), 7.77$ (d, $J=1.1$ $\mathrm{Hz}, 1 \mathrm{H}$, thymine vinyl-CH), $7.34-7.21(\mathrm{~m}, 5 \mathrm{H}, \mathrm{Ar}-\mathrm{H}), 6.85(\mathrm{~d}, J=16.6 \mathrm{~Hz}, 1 \mathrm{H}$, vinyl $\mathrm{C}-\mathrm{H}), 6.43$ (d, $J=16.5 \mathrm{~Hz}, 1 \mathrm{H}$, vinyl C-H), 5.64 (tt, $J=11.5,6.8 \mathrm{~Hz}, 1 \mathrm{H}$, cyclopentane- $\mathrm{NCH}), 3.74(\mathrm{~s}, 3 \mathrm{H}$, ester methyl), $3.52\left(\mathrm{~s}, 3 \mathrm{H}\right.$, ester methyl), 3.23 (dd, $J=15.1,11.3 \mathrm{~Hz}, 1 \mathrm{H}$, cyclopentane $-\mathrm{CH}_{2}$ ), $2.82\left(\mathrm{t}, J=12.1 \mathrm{~Hz}, 1 \mathrm{H}\right.$ cyclopentane $-\mathrm{CH}_{2}$ ), 2.30 (dd, $J=12.5,7.0 \mathrm{~Hz}, 1 \mathrm{H}$, cyclopentane $\mathrm{CH}_{2}$ ), $2.17\left(\mathrm{dd}, J=15.1,6.5 \mathrm{~Hz}, 1 \mathrm{H}\right.$, cyclopentane $\left.-\mathrm{CH}_{2}\right), 1.92(\mathrm{~d}, J=1.2 \mathrm{~Hz}, 3 \mathrm{H}$, thymine methyl), 1.18 (m, $J=1.5 \mathrm{~Hz}, 3 \mathrm{H}$, TIPS), 1.01 (d, $J=2.5 \mathrm{~Hz}, 12 \mathrm{H}$, TIPS), 0.96 (dd, $J=3.4,1.8$ $\mathrm{Hz}, 6 \mathrm{H}, \mathrm{TIPS})$.

${ }^{13} \mathrm{C}$ NMR (101 MHz, Chloroform- $d$, Major diastereoisomer) $\delta 172.4,168.8,163.7,151.1,137.6$, 136.1, 130.7, 128.8, 128.8, 128.2, 126.7, 111.5, 86.7, 69.9, 53.1, 52.6, 51.4, 41.1, 37.1, 29.7, $18.2,18.0,13.1,12.8$.

TIPS methyls are giving 2 differents signals(18.2 and 18.0).

IR 2947 (w), $2868(w), 1689(m), 1465(w), 1435(w), 1260(m), 1088(w), 1017(w), 976(w)$, $911(\mathrm{~m}), 732(\mathrm{~s})$.

HRMS (ESI) calcd for $\mathrm{C}_{31} \mathrm{H}_{45} \mathrm{~N}_{2} \mathrm{O}_{7} \mathrm{Si}^{+}[\mathrm{M}+\mathrm{H}]^{+}$585.2991; found 585.3015.

\section{Dimethyl-3-methyl-4-(5-methyl-2,4-dioxo-3,4-dihydropyrimidin-1(2H)-yl)-2-phenyl-2- ((triisopropylsilyl)oxy)cyclopentane-1,1-dicarboxylate. (24f)}

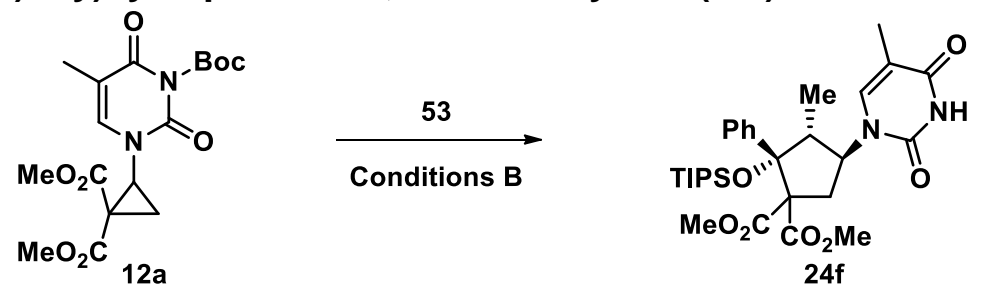

Following the conditions $B$, using triisopropyl((1-phenylprop-1-en-1-yl)oxy)silane (53) $(0.14 \mathrm{~g}$, $0.48 \mathrm{mmol}, 1.2$ equiv), the pure product $24 \mathrm{f}(0.18 \mathrm{~g}, 0.32 \mathrm{mmol}, 79 \%$ yield $)$ was obtained as a shiny foamy solid. The stereochemistry of the methyl was determined by NOE experiments.

$\mathbf{R F}($ pent/AcOET $(1: 1))=0.48$.

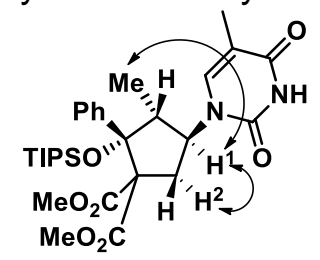

MP $127.4-128.3^{\circ} \mathrm{C}$.

${ }^{1} \mathrm{H}$ NMR $(400 \mathrm{MHz}$, Chloroform- $d) \delta 8.06(\mathrm{~s}, 1 \mathrm{H}$, thymine $\mathrm{NH}), 8.01(\mathrm{~d}, J=1.5 \mathrm{~Hz}, 1 \mathrm{H}$, thymine vinyl-CH), $7.73-7.66(\mathrm{~m}, 2 \mathrm{H}, \mathrm{Ar}-\mathrm{H}), 7.39-7.25(\mathrm{~m}, 3 \mathrm{H}, \mathrm{Ar}-\mathrm{H}), 5.36$ (td, $J=11.4,6.8 \mathrm{~Hz}, 1 \mathrm{H}$ cyclopentane - $\mathrm{NCH}$ ), $3.70(\mathrm{~s}, 3 \mathrm{H}$, ester methyl), $3.35(\mathrm{dq}, J=13.6,6.8 \mathrm{~Hz}, 1 \mathrm{H}$, tetrahydrofuran$\mathrm{CH}$ ), $3.20\left(\mathrm{~s}, 3 \mathrm{H}\right.$, ester methyl), $3.12\left(\mathrm{dd}, J=15.1,11.3 \mathrm{~Hz}, 1 \mathrm{H}\right.$, cyclopentane $\left.-\mathrm{CH}_{2}\right) .2 .33$ (dd, $J=15.1,6.8 \mathrm{~Hz}, 1 \mathrm{H}$, cyclopentane $\left.-\mathrm{CH}_{2}\right), 2.02(\mathrm{~d}, J=1.2 \mathrm{~Hz}, 3 \mathrm{H}$, thymine methyl), $1.56(\mathrm{~s}$, $3 \mathrm{H}$, cyclopentane methyl), 1.24 (hept, $J=7.3 \mathrm{~Hz}, 3 \mathrm{H}$, TIPS), $1.12-0.94$ (m, 18H, TIPS). 
${ }^{13} \mathrm{C}$ NMR (101 MHz, Chloroform- $d$ ) $\delta 172.7,168.1,163.3,151.5,138.6,137.5,128.3,128.2$, $127.4,111.6,90.9,70.4,56.7,52.8,45.2,35.8,18.9,18.8,15.2,12.9,10.6$.

IR 3175 (w), 2963 (w), 2870 (w), $1679(\mathrm{~m}), 1468(w), 1257(\mathrm{~m}), 1079(\mathrm{~m}), 1026(\mathrm{~m}), 910(\mathrm{~m})$, $731(s)$.

HRMS (ESI) calcd for $\mathrm{C}_{30} \mathrm{H}_{45} \mathrm{~N}_{2} \mathrm{O}_{7} \mathrm{Si}^{+}[\mathrm{M}+\mathrm{H}]^{+}$573.2991; found 573.2992.

\subsection{From uracil and 5-fluoro uracil cyclopropanes.}

\section{General procedure for Benzoyl removal.}

The crude product was dissolved in $\mathrm{EtOH}(2 \mathrm{~mL})$ and stirred at room temperature for 2 hours with $\mathrm{NH}_{4} \mathrm{OH}(0.6 \mathrm{~mL}, 40$ equiv, $25 \%)$. The mixture was evaporated to dryness and directly submitted to the column chromatography using a gradient of solvent from pentane/AcOEt (7:3) up to (3:7).

Dimethyl -5-(2,4-dioxo-3,4-dihydropyrimidin-1(2H)-yl)-2-phenyldihydrofuran-3,3(2H)dicarboxylate (26a).
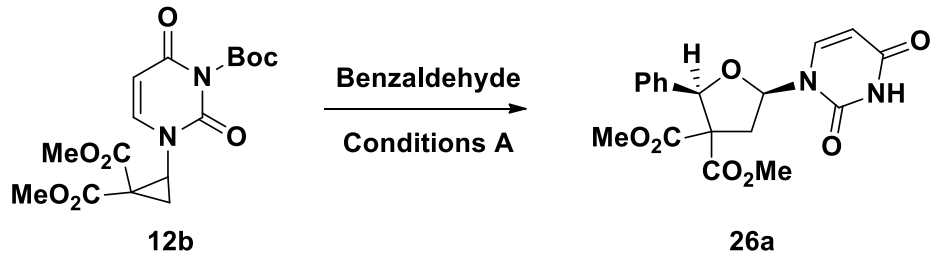

Following the conditions $A$, using benzaldehyde $(0.051 \mathrm{~g}, 0.48 \mathrm{mmol}, 1.2$ equiv) and the corresponding cyclopropane $12 \mathrm{~b}(0.15 \mathrm{~g}, 0.40 \mathrm{mmol}, 1$ equiv), the pure product $26 \mathrm{a}(0.093 \mathrm{~g}$, $0.25 \mathrm{mmol}, 62 \%$ yield) was obtained as a white powder.

$\mathbf{R F}(\mathrm{AcOEt})=0.65$.

MP $209.0-210.7^{\circ} \mathrm{C}$.

${ }^{1} \mathrm{H}$ NMR $(400 \mathrm{MHz}$, Chloroform-d) $\delta 9.07(\mathrm{~s}, 1 \mathrm{H}, \mathrm{NH}), 8.05(\mathrm{~d}, J=8.2 \mathrm{~Hz}, 1 \mathrm{H}$, uracil CH), 7.42 - $7.29(\mathrm{~m}, 2 \mathrm{H}, \mathrm{Ar}-\mathrm{H}), 7.24(\mathrm{~m}, 3 \mathrm{H}, \mathrm{Ar}-\mathrm{H}), 6.29(\mathrm{t}, J=7.4 \mathrm{~Hz}, 1 \mathrm{H}$, tetrahydrofuran $\mathrm{N}-\mathrm{CH}), 5.84$ (d, $J=8.1 \mathrm{~Hz}, 1 \mathrm{H}$, uracil $\mathrm{CH}), 5.52(\mathrm{~s}, 1 \mathrm{H}$, tetrahydrofuran- $\mathrm{CH}), 3.72\left(\mathrm{~s}, 3 \mathrm{H}\right.$, ester $\left.\mathrm{CH}_{3}\right), 3.03$ $\left(\mathrm{s}, 3 \mathrm{H}\right.$, ester $\mathrm{CH}_{3}$ ), $2.83\left(\mathrm{dd}, J=14.7,7.2 \mathrm{~Hz}, 1 \mathrm{H}\right.$, tetrahydrofuran- $\left.\mathrm{CH}_{2}\right), 2.73(\mathrm{dd}, J=14.7,7.8$ $\mathrm{Hz}, 1 \mathrm{H}$, tetrahydrofuran- $\mathrm{CH}_{2}$ ).

${ }^{13}$ C NMR (101 MHz, Chloroform- $\left.d\right) \delta 170.0,169.7,163.0,150.5,140.4,135.1,128.8,128.1$, $126.5,103.6,82.6,81.6,63.8,53.2,52.8,39.4$.

IR 3100 (w), 2968 (w), 1727 (s), 1694 (s), 1459 (m), 1275 (s), 1077 (s), 1052 (s), 730 (s).

HRMS (ESI) calcd for $\mathrm{C}_{18} \mathrm{H}_{18} \mathrm{~N}_{2} \mathrm{NaO}_{7}^{+}[\mathrm{M}+\mathrm{Na}]^{+}$397.1006; found 397.1004.

Dimethyl -5-(5-fluoro-2,4-dioxo-3,4-dihydropyrimidin-1(2H)-yl)-2-phenyldihydrofuran$3,3(2 \mathrm{H})$-dicarboxylate (26b). 

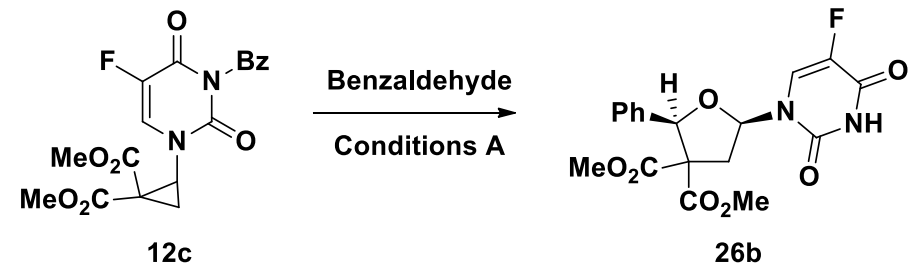

Following the conditions $A$, using benzaldehyde $(0.051 \mathrm{~g}, 0.48 \mathrm{mmol}, 1.2$ equiv), and the corresponding cyclopropane $12 \mathrm{c}(0.16 \mathrm{~g}, 0.40 \mathrm{mmol}, 1 \mathrm{equiv})$, followed by the benzoyl deprotection, the pure product $26 \mathrm{~b}(0.11 \mathrm{~g}, 0.29 \mathrm{mmol}, 72 \%$ yield) was obtained as a white powder.

$\mathbf{R F}($ AcOEt/pentane $(1: 1))=0.60$.

MP $227.9-228.5^{\circ} \mathrm{C}$.

${ }^{1} \mathrm{H}$ NMR $(400 \mathrm{MHz}$, Chloroform- $d) \delta 9.22(\mathrm{~s}, 1 \mathrm{H}, \mathrm{NH}), 8.25(\mathrm{~d}, J=6.1 \mathrm{~Hz}, 1 \mathrm{H}, \mathrm{F}$-uracil $\mathrm{CH})$, $7.47-7.34(\mathrm{~m}, 2 \mathrm{H}, \mathrm{ArH}), 7.34-7.22(\mathrm{~m}, 3 \mathrm{H}, \mathrm{ArH}), 6.31$ (ddd, $J=7.8,6.9,1.7 \mathrm{~Hz}, 1 \mathrm{H}$, tetrahydrofuran $\mathrm{N}-\mathrm{CH}), 5.53(\mathrm{~s}, 1 \mathrm{H}$, tetrahydrofuran $\mathrm{CH}), 3.74\left(\mathrm{~s}, 3 \mathrm{H}\right.$, ester $\left.\mathrm{CH}_{3}\right), 3.08(\mathrm{~s}, 3 \mathrm{H}$, ester $\mathrm{CH}_{3}$ ), 2.80 (qd, $J=14.8,7.4 \mathrm{~Hz}, 2 \mathrm{H}$, tetrahydrofuran $\mathrm{CH}_{2}$ ).

${ }^{13} \mathrm{C}$ NMR (101 MHz, Chloroform- $d$ ) $\delta$ 169.9, 169.6, 156.6 (dd, $\left.J=27.1,8.7 \mathrm{~Hz}\right), 149.1$ (d, $J=$ $7.7 \mathrm{~Hz}$ ), 141.0 (d, $J=238.8 \mathrm{~Hz}$ ), 134.8, 129.0, 128.2, 126.4, 124.7 (d, $J=34.8 \mathrm{~Hz}), 82.7,81.8$, $63.6,53.2,53.0,39.2$.

IR 3196 (w), $3071(w), 2956$ (w), 1723 (s), $1668(\mathrm{~m}), 1436$ (w), $1273(\mathrm{~s}), 1211(\mathrm{~m}), 1094(\mathrm{~m})$, $1053(\mathrm{~m}), 914(\mathrm{~m}), 735(\mathrm{~m})$.

HRMS (ESI) calcd for $\mathrm{C}_{18} \mathrm{H}_{17} \mathrm{FN}_{2} \mathrm{NaO}_{7}[\mathrm{M}+\mathrm{Na}]$ 415.0917; found 415.0918.

\section{Dimethyl-5-(2,4-dioxo-3,4-dihydropyrimidin-1(2H)-yl)-2-methyl-2-phenyldihydrofuran-} $3,3(2 \mathrm{H})$-dicarboxylate (26c).

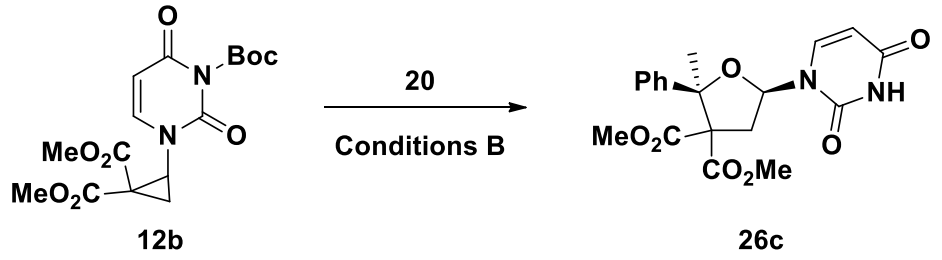

Following the conditions $\mathrm{B}$, using acetophenone $(\mathbf{2 0})(0.058 \mathrm{~g}, 0.48 \mathrm{mmol}, 1.2$ equiv) and the cyclopropane 41 ( $0.16 \mathrm{~g}, 0.40 \mathrm{mmol}, 1$ equiv), the pure product $26 \mathrm{c}(0.12 \mathrm{~g}, 0.30 \mathrm{mmol}, 76 \%$ yield) was obtained as a white powder.

$\mathbf{R F}($ AcOEt/pent $(1: 1))=0.2$.

MP $235.8-238.4^{\circ} \mathrm{C}$.

${ }^{1} \mathrm{H}$ NMR $\left(400 \mathrm{MHz}, \mathrm{DMSO}-d_{6}\right) \delta 11.44(\mathrm{~s}, 1 \mathrm{H}, \mathrm{NH}), 8.18(\mathrm{~d}, J=8.2 \mathrm{~Hz}, 1 \mathrm{H}$, uracil CH), $7.71-$ $7.51(\mathrm{~m}, 2 \mathrm{H}, \mathrm{ArH}), 7.46-7.23(\mathrm{~m}, 3 \mathrm{H}, \mathrm{ArH}), 6.29(\mathrm{dd}, J=8.2,5.1 \mathrm{~Hz}, 1 \mathrm{H}$, tetrahydrofurane N$\mathrm{CH}$ ), 5.81 (dd, $J=8.2,2.2 \mathrm{~Hz}, 1 \mathrm{H}$, uracil $\mathrm{CH}$ ), $3.72\left(\mathrm{~s}, 3 \mathrm{H}\right.$, ester $\left.\mathrm{CH}_{3}\right), 3.27$ (dd, $J=14.8,8.2$ $\mathrm{Hz}, 1 \mathrm{H}$, tetrahydrofurane- $\left.\mathrm{CH}_{2}\right), 3.03\left(\mathrm{~s}, 3 \mathrm{H}\right.$, ester $\left.\mathrm{CH}_{3}\right), 2.67(\mathrm{dd}, J=14.8,5.2 \mathrm{~Hz}, 1 \mathrm{H}$, tetrahydrofurane- $\left.\mathrm{CH}_{2}\right), 1.80\left(\mathrm{~s}, 3 \mathrm{H}\right.$, tetrahydrofurane $\left.-\mathrm{CH}_{3}\right)$.

${ }^{13} \mathrm{C}$ NMR $\left(101 \mathrm{MHz}\right.$, DMSO- $\left.d_{6}\right) \delta 170.6,168.8,163.6,151.1,141.1,141.1,128.2,126.0,102.3$, 87.9, 81.2, 67.0, 53.2, 52.9, 25.8.

Two carbons are unresolved. 
IR 3163 (w), 3035 (w), 2953 (w), 2838 (w), 1735 (s), 1733 (s), 1673 (s), 1436 (m), 1385 (m), 1263 (s), 1210 (s), 1072 (s), 767 (s).

HRMS (ESI) calcd for $\mathrm{C}_{19} \mathrm{H}_{20} \mathrm{~N}_{2} \mathrm{NaO}_{7}^{+}[\mathrm{M}+\mathrm{Na}]^{+} 411.1163$; found 411.1168 .

Dimethyl -5-(5-fluoro-2,4-dioxo-3,4-dihydropyrimidin-1(2H)-yl)-2-methyl-2phenyldihydrofuran-3,3(2H)-dicarboxylate (26d).

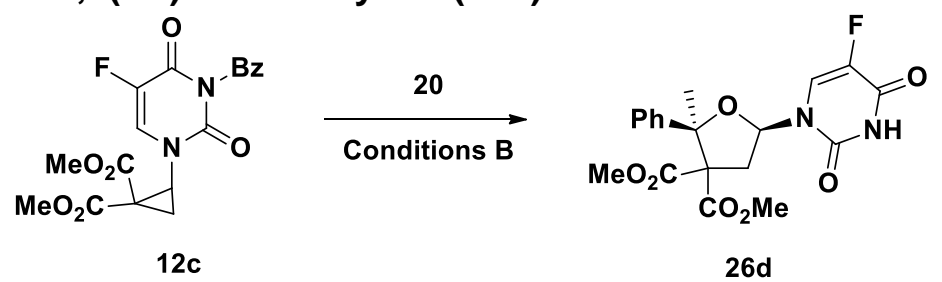

Following the conditions $\mathrm{B}$, using acetophenone $(\mathbf{2 0})(0.058 \mathrm{~g}, 0.48 \mathrm{mmol}, 1.2$ equiv) and the corresponding cyclopropane $12 \mathrm{c}\left(0.16 \mathrm{~g}, 0.40 \mathrm{mmol}, 1\right.$ equiv) at $-40{ }^{\circ} \mathrm{C}$, followed by the benzoyl deprotection, the pure product $26 \mathrm{~d}(0.12 \mathrm{~g}, 0.28 \mathrm{mmol}, 71 \%$ yield $)$ was obtained as a white powder.

$\mathbf{R F}($ AcOEt/pentane $(1: 1))=0.42$.

MP 209.3-210.2 ${ }^{\circ} \mathrm{C}$.

${ }^{1} \mathrm{H}$ NMR $(400 \mathrm{MHz}$, Chloroform- $d) \delta 9.98(\mathrm{~d}, J=4.6 \mathrm{~Hz}, 1 \mathrm{H}, \mathrm{NH}), 8.50(\mathrm{~d}, J=6.5 \mathrm{~Hz}, 1 \mathrm{H}, \mathrm{F}-$ uracil $\mathrm{CH}$ ), $7.77-7.65(\mathrm{~m}, 2 \mathrm{H}, \mathrm{ArH}), 7.51-7.16(\mathrm{~m}, 3 \mathrm{H}, \mathrm{ArH}), 6.42$ (ddd, $J=8.2,5.1,1.7 \mathrm{~Hz}$, $1 \mathrm{H}$, tetrahydrofuran $\mathrm{N}-\mathrm{CH}), 3.80\left(\mathrm{~s}, 3 \mathrm{H}\right.$, ester $\left.\mathrm{CH}_{3}\right), 3.18(\mathrm{dd}, \mathrm{J}=15.1,8.2 \mathrm{~Hz}, 1 \mathrm{H}$, tetrahydrofuran $\mathrm{CH}_{2}$ ), $3.10\left(\mathrm{~s}, 3 \mathrm{H}\right.$, ester $\left.\mathrm{CH}_{3}\right), 2.78(\mathrm{dd}, J=15.1,5.1 \mathrm{~Hz}, 1 \mathrm{H}$, tetrahydrofuran $\left.\mathrm{CH}_{2}\right), 1.88\left(\mathrm{~s}, 3 \mathrm{H}, \mathrm{CH}_{3}\right)$.

${ }^{13} \mathrm{C}$ NMR $(101 \mathrm{MHz}$, Chloroform- $d) \delta 170.5,168.6,157.1(\mathrm{~d}, J=26.8 \mathrm{~Hz}), 149.6,140.5(\mathrm{~d}, J=$ 236.4 Hz), 140.0, 128.0, 127.9, 125.7, 125.6 (d, J = 35.1 Hz), 88.6, 81.4, 66.9, 52.9, 52.8, 39.7, 25.7.

IR 3173 (w), 3065 (w), 2954 (w), 1758 (w), 1718 (s), 1669 (s), 1485 (w), $1421(w), 1262$ (s), $1205(\mathrm{~m}), 1076(\mathrm{~s}), 914(\mathrm{~m}), 768(\mathrm{~m})$.

HRMS (ESI) calcd for $\mathrm{C}_{19} \mathrm{H}_{19} \mathrm{FN}_{2} \mathrm{NaO}_{7}[\mathrm{M}+\mathrm{Na}]$ 429.1074; found 429.1080.

Dimethyl-4-(2,4-dioxo-3,4-dihydropyrimidin-1(2H)-yl)-2-phenyl-2((triisopropylsilyl)oxy)cyclopentane-1,1-dicarboxylate (26e).

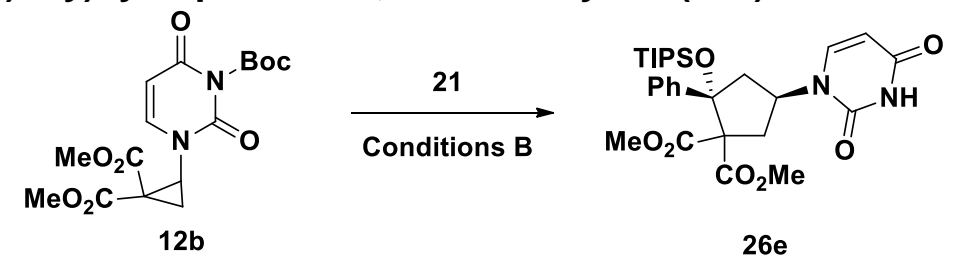

Following the conditions B, using TIPS protected acetophenone $21(0.17 \mathrm{~g}, 0.60 \mathrm{mmol}, 1.5$ equiv) and the cyclopropane $12 \mathrm{~b}(0.16 \mathrm{~g}, 0.40 \mathrm{mmol}$, 1 equiv), the pure product $26 \mathrm{e}(0.18 \mathrm{~g}$, $0.33 \mathrm{mmol}, 81 \%$ yield) was obtained as a colorless foam.

$\mathbf{R F}(\operatorname{AcOEt} /$ pent $(1: 1))=0.5$.

MP $67.7-77.0^{\circ} \mathrm{C}$. 
${ }^{1} \mathrm{H}$ NMR $(400 \mathrm{MHz}$, Chloroform- $d) \delta 9.92(\mathrm{~s}, 1 \mathrm{H}, \mathrm{NH}), 8.11(\mathrm{~d}, J=8.1 \mathrm{~Hz}, 1 \mathrm{H}$, uracil CH), 7.85 - $7.65(\mathrm{~m}, 2 \mathrm{H}, \mathrm{ArH}), 7.30(\mathrm{~m}, 3 \mathrm{H}, \mathrm{ArH}), 5.86(\mathrm{dd}, J=8.1,2.2 \mathrm{~Hz}, 1 \mathrm{H}$, uracil $\mathrm{CH}), 5.74$ (tt, $J=$ 11.3, $7.0 \mathrm{~Hz}, 1 \mathrm{H}$, cyclopentane $\mathrm{N}-\mathrm{CH}$ ), 3.76 (s, 3H ester methyl), 3.34 (dd, $J=15.1,11.1 \mathrm{~Hz}$, $1 \mathrm{H}$, cyclopentane $\mathrm{CH}_{2}$ ), 3.29 (s, $3 \mathrm{H}$, ester methyl), $3.18\left(\mathrm{t}, \mathrm{J}=12.4 \mathrm{~Hz}, 1 \mathrm{H}\right.$, cyclopentane $\mathrm{CH}_{2}$ ), $2.55\left(\mathrm{dd}, J=12.9,6.8 \mathrm{~Hz}, 1 \mathrm{H}\right.$, cyclopentane $\left.\mathrm{CH}_{2}\right), 2.37(\mathrm{dd}, J=15.1,7.1 \mathrm{~Hz}, 1 \mathrm{H}$, cyclopentane $\left.\mathrm{CH}_{2}\right), 1.02-0.95(\mathrm{~m}, 21 \mathrm{H}, \mathrm{TIPS})$.

${ }^{13} \mathrm{C}$ NMR (101 MHz, Chloroform- $d$ ) $\delta$ 172.7, 168.1, 163.7, 151.4, 141.7, 139.8, 128.4, 128.0, $127.4,103.2$, 88.3, 70.3, 52.8, 52.3, 51.5, 43.5, 38.2, 18.2, 18.2, 13.8 .

IR 3183 (w), 3060 (w), 2950 (w), 1686 (s), 1462 (m), $1261(\mathrm{~m}), 1113(w), 990(w), 885(w)$.

HRMS (ESI) calcd for $\mathrm{C}_{28} \mathrm{H}_{40} \mathrm{~N}_{2} \mathrm{NaO}_{7} \mathrm{Si}^{+}[\mathrm{M}+\mathrm{Na}]^{+}$567.2497; found 567.2496.

\section{Dimethyl -4-(5-fluoro-2,4-dioxo-3,4-dihydropyrimidin-1(2H)-yl)-2-phenyl-2-} ((triisopropylsilyl)oxy)cyclopentane-1,1-dicarboxylate (26f).

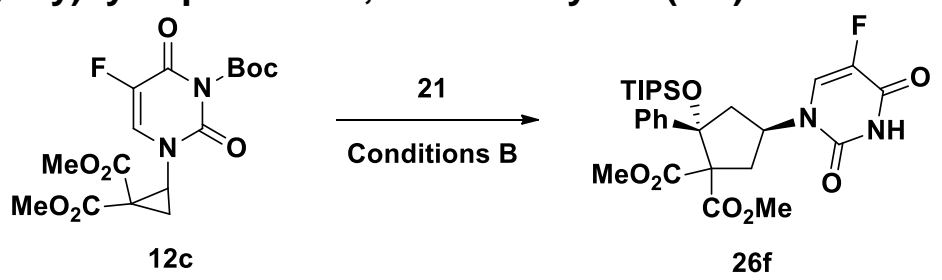

Following the conditions B, using TIPS protected acetophenone $21(0.17 \mathrm{~g}, 0.60 \mathrm{mmol}, 1.5$ equiv) and the corresponding cyclopropane $12 \mathrm{c}\left(0.16 \mathrm{~g}, 0.40 \mathrm{mmol}, 1\right.$ equiv) at $-40{ }^{\circ} \mathrm{C}$, followed by the benzoyl deprotection, the pure product $26 \mathrm{f}(0.12 \mathrm{~g}, 0.20 \mathrm{mmol}, 51 \%$ yield $)$ was obtained as a colorless oil.

$\mathbf{R F}(\operatorname{AcOEt} /$ pentane $(1: 1))=0.75$

${ }^{1} \mathrm{H}$ NMR (400 MHz, Chloroform-d) $\delta 9.96$ (br. m., $\left.1 \mathrm{H}, \mathrm{NH}\right), 8.33$ (d, $J=6.5 \mathrm{~Hz}, 1 \mathrm{H}$, F-uracil $\mathrm{CH}$ ), 7.76 (dd, $J=7.8,2.0 \mathrm{~Hz}, 2 \mathrm{H}, \mathrm{ArH}), 7.39-7.21(\mathrm{~m}, 3 \mathrm{H}, \mathrm{ArH}), 5.74$ (dddd, $J=8.6,6.5$, 4.6, $1.5 \mathrm{~Hz}, 1 \mathrm{H}$, tetrahydrofuran $\mathrm{N}-\mathrm{CH}), 3.74\left(\mathrm{~s}, 3 \mathrm{H}\right.$, ester $\left.\mathrm{CH}_{3}\right), 3.39-3.29(\mathrm{~m}, 1 \mathrm{H}$, tetrahydrofuran $\left.\mathrm{CH}_{2}\right), 3.28\left(\mathrm{~s}, 3 \mathrm{H}\right.$, ester $\left.\mathrm{CH}_{3}\right), 3.14(\mathrm{dd}, J=13.0,11.7 \mathrm{~Hz}, 1 \mathrm{H}$, tetrahydrofuran $\mathrm{CH}_{2}$ ), 2.55 (dd, $J=12.9,7.0 \mathrm{~Hz}, 1 \mathrm{H}$, tetrahydrofuran $\mathrm{CH}_{2}$ ), $2.35(\mathrm{dd}, J=15.2,6.7 \mathrm{~Hz}, 1 \mathrm{H}$, tetrahydrofuran $\left.\mathrm{CH}_{2}\right), 1.01-0.94(\mathrm{~m}, 21 \mathrm{H}$, TIPS).

${ }^{13} \mathrm{C}$ NMR (101 MHz, Chloroform- $d$ ) $\delta 172.8,168.0,157.0$ (d, $\left.J=26.5 \mathrm{~Hz}\right), 150.2,141.0$ (d, $J=$ $237.5 \mathrm{~Hz}$ ), 139.6, 128.5, 127.9, 127.4, 125.9 (d, $J=33.4 \mathrm{~Hz}), 88.4,70.3,52.9,52.4,52.0$, 43.4, $38.0,18.2,18.2,13.9$.

IR 3194 (w), 3067 (w), 2951 (w), 2855 (w), 1718 (s), 1466 (w), 1256 (s), $1134(\mathrm{~m}), 991$ (m), $788(\mathrm{~m}), 740(\mathrm{~m})$.

HRMS (ESI) calcd for $\mathrm{C}_{28} \mathrm{H}_{39} \mathrm{FN}_{2} \mathrm{NaO}_{7} \mathrm{Si}^{+}[\mathrm{M}+\mathrm{Na}]^{+}$585.2403; found 585.2386. 


\section{Scope of the reaction [4+2] reaction.}

\section{c) General conditions the [4+2] annulation}
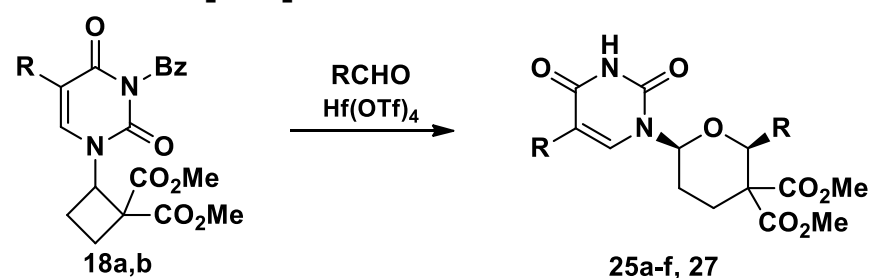

25a-f, 27

$\mathrm{Hf}(\mathrm{OTf})_{4}(21 \mathrm{mg}, 0.030 \mathrm{mmol}, 0.1$ equiv) was added in a flame dried $25 \mathrm{~mL}$ flask and under nitrogen atmosphere. Then, dry dichloromethane $(4.5 \mathrm{~mL})$ and aldehyde $5(0.45 \mathrm{mmol}, 1.5$ equiv) were added into the flask. A solution of cyclobutane $18 \mathbf{a}$ or $18 \mathbf{b}(0.30 \mathrm{mmol}, 1.0$ equiv) in dry dichloromethane $(1.5 \mathrm{~mL})$ was added dropwise to the reaction mixture and stirred at room temperature for 15 minutes. The reaction mixture was filtrated on a pad of silica eluting with a mixture of ethyl acetate/ pentane (8:2) and concentrated under reduced pressure. The crude product was dissolved in ethanol $(6 \mathrm{~mL})$ and ammonium hydroxide solution $(1.8 \mathrm{~mL}, 60$ equiv, $25 \%$ ) was added. The resulting solution was stirred for 16 hours. The solvent was removed under reduced pressure and the crude product was dissolved in ethyl acetate (25 $\mathrm{mL})$ and extracted with water $(3 \times 50 \mathrm{~mL})$. The organic layer was dried over magnesium sulfate, filtrated and concentrated under reduced pressure. The crude mixture was purified by column chromatography eluting with a mixture of ethyl acetate/ pentane (5:5 to 9:1). The product was recrystallized in ethanol $(2 \mathrm{~mL})$.

\section{Dimethyl (5-methyl-2,4-dioxo-3,4-dihydropyrimidin-1(2H)-yl)-2-phenyldihydro-2H- pyran-3,3(4H)-dicarboxylate (25a).}
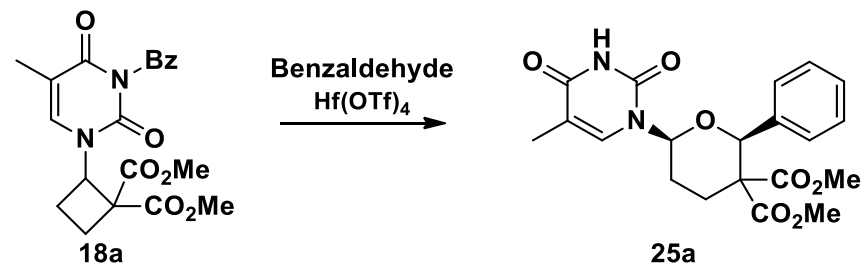

Following the general procedure, using benzaldehyde (5a) (46 $\mu \mathrm{L}, 0.45 \mathrm{mmol}, 1.5$ equiv) cyclobutane 18a (120 mg, $0.300 \mathrm{mmol}, 1.0$ equiv), a mixture of diastereoisomers (20:1 by integration of methyl esters at $3.52 \mathrm{ppm}$ and $3.49 \mathrm{ppm}$ ) 25a (102 mg, $0.255 \mathrm{mmol}, 85 \% \mathrm{NMR}$ yield $)^{7}$ was obtained after column chromatography as a colorless foam. After recrystallization in ethanol, the pure product $25 \mathrm{a}$ (94 $\mathrm{mg}, 0.23 \mathrm{mmol}, 78 \%$ isolated yield) was obtained as a colorless foam and as a mixture of diastereoisomers ( $>20: 1$ by integration of methyl esters at $3.52 \mathrm{ppm}$ and $3.49 \mathrm{ppm})$.

RF (Pentane/Ethyl acetate (3:7)) $=.0 .27$.

MP 226.2- $227.9^{\circ} \mathrm{C}$.

${ }^{1} \mathrm{H}$ NMR (400 MHz, Chloroform-d, major diastereoisomer) $\delta 8.38(\mathrm{~s}, 1 \mathrm{H}, \mathrm{NH}), 7.40-7.35$ (m, $2 \mathrm{H}, \mathrm{Ar}-\mathrm{H}), 7.34-7.27(\mathrm{~m}, 4 \mathrm{H}, \mathrm{Ar}-\mathrm{H}$ and thymine $\mathrm{C}=\mathrm{C}-\mathrm{H}), 5.96(\mathrm{dd}, \mathrm{J}=11.1,2.9 \mathrm{~Hz}, 1 \mathrm{H}$, tetrahydropyran- $\mathrm{NCH}), 5.23(\mathrm{~s}, 1 \mathrm{H}$, tetrahydropyran- $\mathrm{CH}), 3.68\left(\mathrm{~s}, 3 \mathrm{H}\right.$, ester $\left.\mathrm{CH}_{3}\right), 3.52(\mathrm{~s}, 3$ $\mathrm{H}$, ester $\left.\mathrm{CH}_{3}\right), 2.74-2.64\left(\mathrm{~m}, 1 \mathrm{H}\right.$, tetrahydropyran $\left.-\mathrm{CH}_{2}\right), 2.38$ (tdd, $J=12.6,11.0,4.1 \mathrm{~Hz}, 1$ $\mathrm{H}$, tetrahydropyran- $\left.\mathrm{CH}_{2}\right), 2.25\left(\mathrm{td}, J=13.4,4.1 \mathrm{~Hz}, 1 \mathrm{H}\right.$, tetrahydropyran- $\left.\mathrm{CH}_{2}\right), 1.99(\mathrm{~d}, J=1.2$ $\mathrm{Hz}, 3 \mathrm{H}$, thymine- $\left.\mathrm{CH}_{3}\right), 1.97-1.90\left(\mathrm{~m}, 1 \mathrm{H}\right.$, tetrahydropyran- $\left.\mathrm{CH}_{2}\right)$.

\footnotetext{
${ }^{7}$ Benzamide as impurity which is not possible to separate by column chromatography.
} 
${ }^{13} \mathrm{C}$ NMR (101 MHz, Chloroform- $d$, major diastereoisomer) $\delta 170.3,169.2,163.4,149.9,137.5$, 135.4, 128.5, 127.7, 127.5, 111.6, 82.3, 82.1, 57.1, 52.8, 52.0, 31.6, 26.6, 12.8.

IR 3191 (w), 3041 (w), 2954 (w), 2361 (w), 1725 (s), 1693 (s), 1461 (w), 1271 (s), 1096 (w), $1043(w), 915(w), 735(m)$.

HRMS (ESI) calcd for $\mathrm{C}_{20} \mathrm{H}_{22} \mathrm{~N}_{2} \mathrm{NaO}_{7}^{+}[\mathrm{M}+\mathrm{Na}]^{+} 425.1319$, found 425.1324.

Dimethyl 2-(4-fluorophenyl)-6-(5-methyl-2,4-dioxo-3,4-dihydropyrimidin-1(2H)yl)dihydro-2H-pyran-3,3(4H)-dicarboxylate (25b).

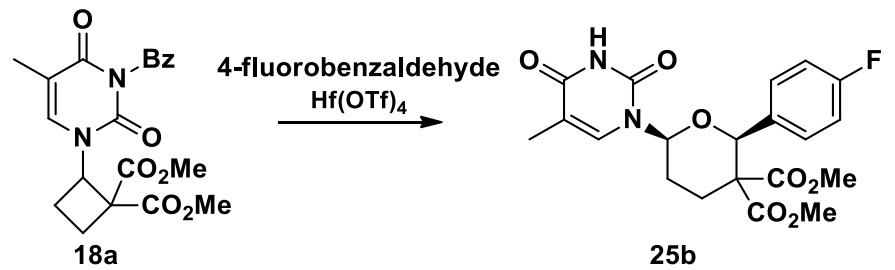

Following the general procedure, using 4-fluorobenzaldehyde (5f) (48 $\mu \mathrm{L}, 0.45 \mathrm{mmol}, 1.5$ equiv) cyclobutane $18 \mathrm{a}$ (120 mg, $0.300 \mathrm{mmol}, 1.0$ equiv), a mixture of diastereoisomers (12:1 by integration of methyl esters at $3.54 \mathrm{ppm}$ and $3.49 \mathrm{ppm}) \mathbf{2 5 b}(99 \mathrm{mg}, 0.24 \mathrm{mmol}, 79 \% \mathrm{NMR}$ yield $)^{7}$ was obtained after column chromatography as a colorless foam. After recrystallization in ethanol, the pure product $25 \mathrm{~b}$ (88 $\mathrm{mg}, 0.21 \mathrm{mmol}, 70 \%$ isolated yield) was obtained as a colorless foam and as a mixture of diastereoisomers (12:1 by integration of methyl esters at $3.54 \mathrm{ppm}$ and $3.49 \mathrm{ppm})$.

RF (Pentane/Ethyl acetate $(3: 7))=0.30$.

MP $94.8-96.9^{\circ} \mathrm{C}$.

${ }^{1} \mathbf{H}$ NMR (400 MHz, Chloroform-d, major diastereoisomer) $\delta 8.06(\mathrm{~s}, 1 \mathrm{H}, \mathrm{NH}), 7.40-7.33(\mathrm{~m}$, $2 \mathrm{H}, \mathrm{Ar}-\mathrm{H}), 7.29(\mathrm{~d}, J=1.3 \mathrm{~Hz}, 1 \mathrm{H}$, thymine $\mathrm{C}=\mathrm{C}-\mathrm{H}), 7.03-6.95(\mathrm{~m}, 2 \mathrm{H}, \mathrm{Ar}-\mathrm{H}), 5.95(\mathrm{dd}, \mathrm{J}=$ $11.3,2.9 \mathrm{~Hz}, 1 \mathrm{H}$, tetrahydropyran- $\mathrm{NCH}), 5.21(\mathrm{~s}, 1 \mathrm{H}$, tetrahydropyran- $\mathrm{CH}), 3.69(\mathrm{~s}, 3 \mathrm{H}$, ester $\left.\mathrm{CH}_{3}\right), 3.54\left(\mathrm{~s}, 3 \mathrm{H}\right.$, ester $\left.\mathrm{CH}_{3}\right), 2.74-2.63\left(\mathrm{~m}, 1 \mathrm{H}\right.$, tetrahydropyran- $\left.\mathrm{CH}_{2}\right), 2.48-2.32(\mathrm{~m}, 1 \mathrm{H}$, tetrahydropyran- $\left.\mathrm{CH}_{2}\right), 2.32-2.20\left(\mathrm{~m}, 1 \mathrm{H}\right.$, tetrahydropyran- $\left.\mathrm{CH}_{2}\right), 1.99(\mathrm{~d}, J=1.2 \mathrm{~Hz}, 3 \mathrm{H}$, thymine- $\mathrm{CH}_{3}$ ), $1.93\left(\mathrm{dq}, \mathrm{J}=9.3,3.3 \mathrm{~Hz}, 1 \mathrm{H}\right.$, tetrahydropyran- $\mathrm{CH}_{2}$ ).

${ }^{13} \mathrm{C}$ NMR (101 MHz, Chloroform-d, major diastereoisomer) $\delta 170.3,169.1,163.6,162.5(\mathrm{~d}, J$ $=225.5 \mathrm{~Hz}$ ), 150.2, 135.2, $133.4(\mathrm{~d}, J=3.3 \mathrm{~Hz}), 129.3(\mathrm{~d}, J=8.1 \mathrm{~Hz}), 114.6$ (d, J = 21.4 Hz), $111.8,82.0,81.6,57.0,52.8,52.1,31.4,26.5,12.8$.

IR 3203 (w), 3055 (w), 2961 (w), 2361 (w), 2255 (w), 1724 (s), 1691 (s), 1515 (w), 1464 (w), $1267(\mathrm{~s}), 1229(\mathrm{~m}), 1100(\mathrm{~m}), 1050(\mathrm{~m}), 913(\mathrm{~m}), 735(\mathrm{~m})$.

HRMS (ESI) calcd for $\mathrm{C}^{20} \mathrm{H}^{21} \mathrm{FN}^{2} \mathrm{NaO}^{7+}[\mathrm{M}+\mathrm{Na}]^{+}$443.1225; found 443.1224.

Dimethyl 6-(5-methyl-2,4-dioxo-3,4-dihydropyrimidin-1(2H)-yl)-2-(thiophen-2-yl)dihydro2H-pyran-3,3(4H)-dicarboxylate (25c).
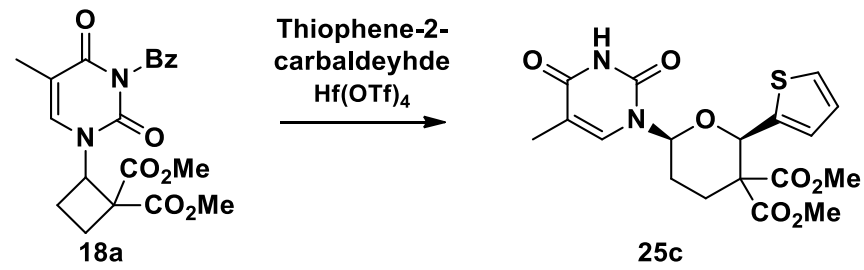

Following the general procedure, using thiophene-2-carbaldehyde ( $42 \mu \mathrm{L}, .45 \mathrm{mmol}, 1.5$ equiv) cyclobutane 18a (120 mg, $0.300 \mathrm{mmol}, 1.0$ equiv), a mixture of diastereoisomers (12:1 by integration of methyl esters at $3.56 \mathrm{ppm}$ and $3.54 \mathrm{ppm}) 25 \mathrm{c}(111 \mathrm{mg}, 0.270 \mathrm{mmol}, 90 \% \mathrm{NMR}$ yield $)^{7}$ was obtained after column chromatography as a white foam. After recrystallization in 
ethanol, the pure product $25 \mathrm{c}$ (97 $\mathrm{mg}, 0.24 \mathrm{mmol}, 79 \%$ isolated yield) was obtained as white foam and as a mixture of diastereoisomers (7:1 by integration of methyl esters at $3.56 \mathrm{ppm}$ and $3.54 \mathrm{ppm})$.

RF (Pentane/Ethyl acetate $(4: 6))=0.24$.

MpP186.4-188. $3^{\circ} \mathrm{C}$.

${ }^{1} \mathrm{H}$ NMR (400 MHz, Chloroform-d, major diastereoisomer) $\delta 9.74(\mathrm{~s}, 1 \mathrm{H}, \mathrm{NH}), 7.27$ (d, $J=1.4$ $\mathrm{Hz}, 1 \mathrm{H}$, , thymine $\mathrm{C}=\mathrm{C}-\mathrm{H}), 7.23(\mathrm{dd}, J=5.1,1.2 \mathrm{~Hz}, 1 \mathrm{H}$, thiophene-H), $7.04(\mathrm{dd}, J=3.5,1.1$ $\mathrm{Hz}, 1 \mathrm{H}$, thiophene $-\mathrm{H}), 6.92(\mathrm{dd}, J=5.1,3.6 \mathrm{~Hz}, 1 \mathrm{H}$, thiophene $-\mathrm{H}), 5.95(\mathrm{dd}, J=10.9,2.8$ $\mathrm{Hz}, 1 \mathrm{H}$, tetrahydropyran- $\mathrm{NCH}), 5.48(\mathrm{~d}, J=0.8 \mathrm{~Hz}, 1 \mathrm{H}$, tetrahydropyran- $\mathrm{CH}), 3.71(\mathrm{~s}, 3 \mathrm{H}$, ester $\left.\mathrm{CH}_{3}\right), 3.56\left(\mathrm{~s}, 3 \mathrm{H}\right.$, ester $\left.\mathrm{CH}_{3}\right), 2.70-2.61\left(\mathrm{~m}, 1 \mathrm{H}\right.$, tetrahydropyran- $\left.\mathrm{CH}_{2}\right), 2.32-2.24$ $\left(\mathrm{m}, 1 \mathrm{H}\right.$, tetrahydropyran- $\left.\mathrm{CH}_{2}\right), 2.23-2.14\left(\mathrm{~m}, 1 \mathrm{H}\right.$, tetrahydropyran- $\left.\mathrm{CH}_{2}\right), 1.95(\mathrm{~d}, J=1.3 \mathrm{~Hz}$, $3 \mathrm{H}$, thymine- $\left.\mathrm{CH}_{3}\right), 1.92\left(\mathrm{dd}, J=7.9,2.3 \mathrm{~Hz}, 1 \mathrm{H}\right.$, tetrahydropyran- $\mathrm{CH}_{2}$ ).

${ }^{13} \mathrm{C}$ NMR (101 MHz, Chloroform-d, major diastereoisomer) $\delta 170.2,168.9,163.6,150.1,139.8$, 135.2, 126.2, 126.2, 125.5, 111.7, 82.1, 79.1, 57.4, 52.9, 52.5, 31.1, 26.5, 12.8.

IR 3195 (w), $3042(w), 2954(w), 2832(w), 2362(w), 2252(w), 2252(w), 1690(s), 1441(w)$, $1375(\mathrm{w}), 1271(\mathrm{~s}), 1103(\mathrm{~m}), 1050(\mathrm{w}), 913(\mathrm{~m}), 734(\mathrm{~m})$.

HRMS (ESI) calcd for $\mathrm{C}_{18} \mathrm{H}_{20} \mathrm{~N}_{2} \mathrm{NaO}_{7} \mathrm{~S}^{+}[\mathrm{M}+\mathrm{Na}]^{+} 431.0883$; found 431.0879 .

Dimethyl 2-(2-methoxyphenyl)-6-(5-methyl-2,4-dioxo-3,4-dihydropyrimidin-1(2H)yl)dihydro-2H-pyran-3,3(4H)-dicarboxylate (25d).
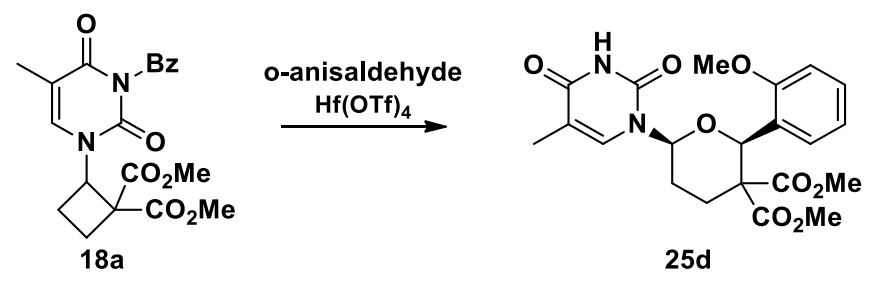

Following the general procedure, using o-anisaldehyde (61 mg, $0.45 \mathrm{mmol}, 1.5$ equiv) and cyclobutane $18 \mathrm{a}$ (120 mg, $0.300 \mathrm{mmol}, 1.0$ equiv), a mixture of diastereoisomers (10:1 by integration of methyl esters at 3.54 ppm and $3.40 \mathrm{ppm}$ ) 25d (108 mg, $0.252 \mathrm{mmol}, 84 \% \mathrm{NMR}$ yield $)^{7}$ was obtained after column chromatography as a white solid. After recrystallization in ethanol, the pure product $25 \mathrm{~d}$ (98 $\mathrm{mg}, 0.23 \mathrm{mmol}, 76 \%$ isolated yield) was obtained as white solid and as a mixture of diastereoisomers (10:1 by integration of methyl esters at $3.54 \mathrm{ppm}$ and $3.40 \mathrm{ppm})$.

RF (Pentane/Ethyl acetate (3:7)) $=0.29$.

MP 193.9- $196.2^{\circ} \mathrm{C}$.

${ }^{1} \mathbf{H}$ NMR (400 MHz, Chloroform-d, major diastereoisomer) $\delta 8.05(\mathrm{~s}, 1 \mathrm{H}, \mathrm{NH}), 7.77$ (dd, $J=$ 7.8, $1.7 \mathrm{~Hz}, 1 \mathrm{H}, \mathrm{Ar}-\mathrm{H}), 7.39(\mathrm{~d}, J=1.4 \mathrm{~Hz}, 1 \mathrm{H}$, thymine $\mathrm{C}=\mathrm{C}-\mathrm{H}), 7.31-7.27(\mathrm{~m}, 1 \mathrm{H}, \mathrm{Ar}-\mathrm{H})$, 7.01 (td, $J=7.6,1.1 \mathrm{~Hz}, 1 \mathrm{H}, \mathrm{Ar}-\mathrm{H}), 6.80(\mathrm{dd}, J=8.3,1.1 \mathrm{~Hz}, 1 \mathrm{H}, \mathrm{Ar}-\mathrm{H}), 5.92(\mathrm{dd}, J=11.0$, $3.0 \mathrm{~Hz}, 1 \mathrm{H}$, tetrahydropyran- $\mathrm{NCH}), 5.29(\mathrm{~s}, 1 \mathrm{H}$, tetrahydropyran- $\mathrm{CH}), 3.77(\mathrm{~s}, 3 \mathrm{H}, \mathrm{O}-\mathrm{Me}$ $\left.\mathrm{CH}_{3}\right), 3.69\left(\mathrm{~s}, 3 \mathrm{H}\right.$, ester $\left.\mathrm{CH}_{3}\right), 3.54\left(\mathrm{~s}, 3 \mathrm{H}\right.$, ester $\left.\mathrm{CH}_{3}\right), 2.63-2.55(\mathrm{~m}, 2 \mathrm{H}$, tetrahydropyran$\left.\mathrm{CH}_{2}\right), 2.05-2.01\left(\mathrm{~m}, 1 \mathrm{H}\right.$, tetrahydropyran- $\left.\mathrm{CH}_{2}\right), 1.99\left(\mathrm{~d}, \mathrm{~J}=1.3 \mathrm{~Hz}, 3 \mathrm{H}\right.$, thymine- $\left.\mathrm{CH}_{3}\right), 1.85$ $-1.74\left(\mathrm{~m}, 1 \mathrm{H}\right.$, tetrahydropyran- $\left.\mathrm{CH}_{2}\right)$.

${ }^{13} \mathrm{C}$ NMR (101 MHz, Chloroform- $d$, major diastereoisomer) $\delta$ 169.0, 168.9, 163.8, 156.2, 150.0, $135.7,129.9,129.4,125.6,120.3,111.5,109.4$, 82.2, 76.4, 56.9, 55.5, 52.5, 52.3, 31.1, 27.3, 12.9 .

IR 3191 (w), 3045 (w), 2955 (w), 2841 (w), 2361 (w), 2338 (w), 2253 (w), 1731 (s), 1690 (s), $1464(\mathrm{~m}), 1377(\mathrm{w}), 1259(\mathrm{~s}), 1104(\mathrm{~m}), 1047(\mathrm{~m}), 914(\mathrm{~m}), 733(\mathrm{~s})$.

HRMS (ESI) calcd for $\mathrm{C}_{21} \mathrm{H}_{24} \mathrm{~N}_{2} \mathrm{NaO}_{8}{ }^{+}[\mathrm{M}+\mathrm{Na}]^{+} 455.1425$; found 455.1429. 

yl)dihydro-2H-pyran-3,3(4H)-dicarboxylate (25e).
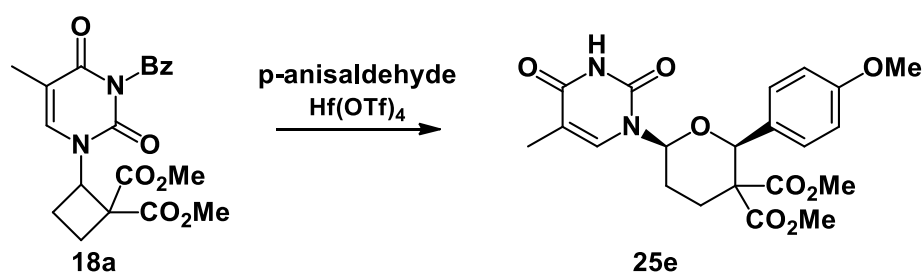

Following the general procedure, using $p$-anisaldehyde (55 $\mu \mathrm{L}, 0.45 \mathrm{mmol}, 1.5$ equiv) and cyclobutane $18 \mathrm{a}$ (120 mg, $0.300 \mathrm{mmol}, 1.0$ equiv), a mixture of diastereoisomers (5:1 by integration of methyl esters at $3.55 \mathrm{ppm}$ and $3.49 \mathrm{ppm}) 25 \mathrm{e}(115 \mathrm{mg}, 0.264 \mathrm{mmol}, 88 \% \mathrm{NMR}$ yield ${ }^{7}$ was obtained after column chromatography as a slightly yellow foam. After recrystallization in ethanol, the pure product $25 \mathrm{e}(99 \mathrm{mg}, 0.22 \mathrm{mmol}, 76 \%$ isolated yield) was obtained as a slightly yellow foam and as a mixture of diastereoisomers (7:1 by integration of methyl esters at $3.55 \mathrm{ppm}$ and $3.49 \mathrm{ppm}$ ).

RF (Pentane/Ethyl acetate (3:7)) $=0.26$.

MP 97.4- $99.5^{\circ} \mathrm{C}$.

${ }^{1} \mathbf{H}$ NMR (400 MHz, Chloroform- $d$, major diastereoisomer) $9.03(\mathrm{~s}, 1 \mathrm{H}, \mathrm{NH}), 7.31(\mathrm{~d}, J=1.3$ $\mathrm{Hz}, 1 \mathrm{H}$, thymine $\mathrm{C}=\mathrm{C}-\mathrm{H}), 7.30-7.27(\mathrm{~m}, 2 \mathrm{H}, \mathrm{Ar}-\mathrm{H}), 6.86-6.78(\mathrm{~m}, 2 \mathrm{H}, \mathrm{Ar}-\mathrm{H}), 5.95(\mathrm{dd}, \mathrm{J}=$ $11.1,2.9 \mathrm{~Hz}, 1 \mathrm{H}$, tetrahydropyran- $\mathrm{NCH}), 5.16(\mathrm{~s}, 1 \mathrm{H}$, tetrahydropyran- $\mathrm{CH}), 3.78(\mathrm{~s}, 3 \mathrm{H}, \mathrm{O}-$ Me $\left.\mathrm{CH}_{3}\right), 3.67\left(\mathrm{~s}, 3 \mathrm{H}\right.$, ester $\left.\mathrm{CH}_{3}\right), 3.55\left(\mathrm{~s}, 3 \mathrm{H}\right.$, ester $\left.\mathrm{CH}_{3}\right), 2.74-2.61(\mathrm{~m}, 1 \mathrm{H}$, tetrahydropyran- $\mathrm{CH}_{2}$ ), 2.35 (tdd, $J=12.6,11.0,4.1 \mathrm{~Hz}, 1 \mathrm{H}$, tetrahydropyran- $\mathrm{CH}_{2}$ ), 2.22 (td, $J$ $=13.4,4.1 \mathrm{~Hz}, 1 \mathrm{H}$, tetrahydropyran- $\left.\mathrm{CH}_{2}\right), 1.97\left(\mathrm{~d}, J=1.3 \mathrm{~Hz}, 3 \mathrm{H}\right.$, thymine- $\left.\mathrm{CH}_{3}\right), 1.95-1.88$ ( $\mathrm{m}, 1 \mathrm{H}$, tetrahydropyran- $\mathrm{CH}_{2}$ ).

${ }^{13} \mathrm{C}$ NMR (101 MHz, Chloroform- $d$, major diastereoisomer) $\delta 170.3,169.3,163.7,159.5,150.2$, 135.4, 129.6, 128.8, 113.0, 111.6, 82.0, 82.0, 57.0, 55.3, 52.7, 52.1, 31.4, 26.6, 12.8.

IR 3191 (w), 3049 (w), 2954 (w), 2839 (w), 2360 (w), 2257 (w), 1687 (s), 1517 (w), $1461(\mathrm{~m})$, $1255(\mathrm{~s}), 1109(\mathrm{~m}), 1044(\mathrm{~m}), 910(\mathrm{~m}), 817(\mathrm{w}), 730(\mathrm{~s})$.

HRMS (ESI) calcd for for $\mathrm{C}_{21} \mathrm{H}_{24} \mathrm{~N}_{2} \mathrm{NaO}_{8}{ }^{+}[\mathrm{M}+\mathrm{Na}]^{+} 455.1425$; found 455.1428.

(E)-dimethyl 6-(5-methyl-2,4-dioxo-3,4-dihydropyrimidin-1(2H)-yl)-2-styryldihydro-2Hpyran-3,3(4H)-dicarboxylate (25f).

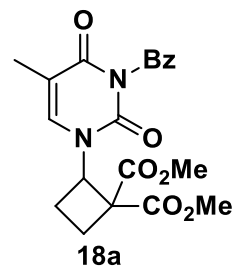
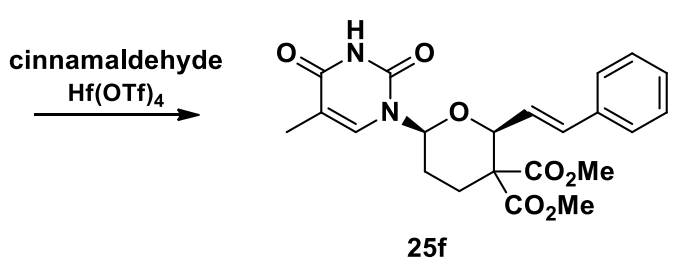

$25 f$

Following the general procedure, using cinnamaldehyde ( $57 \mu \mathrm{L}, 0.45 \mathrm{mmol}, 1.5$ equiv) cyclobutane $18 \mathrm{a}$ (120 mg, $0.300 \mathrm{mmol}, 1.0$ equiv), a mixture of diastereoisomers (2.5:1 by integration of methyl esters at $3.72 \mathrm{ppm}$ and $3.67 \mathrm{ppm}) \mathbf{2 5 f}(104 \mathrm{mg}, 0.243 \mathrm{mmol}, 81 \% \mathrm{NMR}$ yield $)^{7}$ was obtained after column chromatography as a yellow foam. After recrystallization in ethanol, the pure product $25 \mathrm{f}(89 \mathrm{mg}, 0.21 \mathrm{mmol}, 69 \%$ isolated yield) was obtained as yellow foam and as a mixture of diastereoisomers (2.5:1 by integration of methyl esters at $3.72 \mathrm{ppm}$ and $3.67 \mathrm{ppm})$.

RF (Pentane/Ethyl acetate $(3: 7))=0.37$. 
MP $73.7-75-5^{\circ} \mathrm{C}$.

${ }^{1} \mathrm{H}$ NMR (400 MHz, Chloroform- $\left.d\right) \delta 9.03$ (s, 1H, N H, major diastereoisomer), 8.91 (s, $1 \mathrm{H}$, $\mathrm{NH}$, minor diastereoisomer), $7.39-7.21(\mathrm{~m}, 10 \mathrm{H}, \mathrm{Ar}-\mathrm{H}$, major and minor diastereoisomers), 7.18 (dd, $J=3.4,2.0 \mathrm{~Hz}, 2 \mathrm{H}$, thymine $\mathrm{C}=\mathrm{C}-\mathrm{H}$, , major and minor diastereoisomers), 6.79 (dd, $J=15.7,1.1 \mathrm{~Hz}, 1 \mathrm{H}$, vinyl $\mathrm{CH}$, minor diastereoisomer), $6.61-6.49(\mathrm{~m}, 2 \mathrm{H}$, vinyl $\mathrm{CH}$, major diastereoisomer), 6.31 (dd, $J=15.7,8.0 \mathrm{~Hz}, 1 \mathrm{H}$, , vinyl $\mathrm{CH}$, minor diastereoisomer), 6.02 (dd, $J=11.2,2.8 \mathrm{~Hz}, 1 \mathrm{H}$, tetrahydropyran- $\mathrm{NCH}$, minor diastereoisomer), 5.91 (dd, $J=11.0,3.0$ $\mathrm{Hz}, 1 \mathrm{H}$, tetrahydropyran- $\mathrm{NCH}$, major diastereoisomer), $5.40(\mathrm{~d}, J=8.0 \mathrm{~Hz}, 1 \mathrm{H}$, tetrahydropyran- $\mathrm{CH}$, minor diastereoisomer), $4.68(\mathrm{~d}, \mathrm{~J}=5.3 \mathrm{~Hz}, 1 \mathrm{H}$, tetrahydropyran- $\mathrm{CH}$, major diastereoisomer), 3.87 (s, $3 \mathrm{H}$, ester $\mathrm{CH}_{3}$, minor diastereoisomer), 3.78 (s, $3 \mathrm{H}$, ester $\mathrm{CH}_{3}$, major diastereoisomer), $3.71\left(\mathrm{~s}, 3 \mathrm{H}\right.$, ester $\mathrm{CH}_{3}$, major diastereoisomer), $3.66(\mathrm{~s}, 3 \mathrm{H}$, ester $\mathrm{CH}_{3}$, minor diastereoisomer), 2.65 (ddd, $J=13.6,4.2,2.7 \mathrm{~Hz}, 1 \mathrm{H}$, tetrahydropyran- $\mathrm{CH}_{2}$, major diastereoisomer), $2.58-2.35\left(\mathrm{~m}, 2 \mathrm{H}\right.$, tetrahydropyran- $\mathrm{CH}_{2}$, minor diastereoisomer), $2.26-2.15\left(\mathrm{~m}, 1 \mathrm{H}\right.$, tetrahydropyran- $\mathrm{CH}_{2}$, major diastereoisomer $), 2.15-1.98(\mathrm{~m}, 2 \mathrm{H}$, tetrahydropyran- $\mathrm{CH}_{2}$, major diastereoisomer and minor diastereoisomer), $1.95(\mathrm{~d}, J=1.2 \mathrm{~Hz}$, $3 \mathrm{H}$, thymine- $\mathrm{CH}_{3}$, major diastereoisomer), $1.93\left(\mathrm{~d}, \mathrm{~J}=1.3 \mathrm{~Hz}, 3 \mathrm{H}\right.$, thymine- $\mathrm{CH}_{3}$, minor diastereoisomer), $1.92-1.87\left(\mathrm{~m}, 1 \mathrm{H}\right.$, tetrahydropyran- $\mathrm{CH}_{2}$, major diastereoisomer), $1.56-$ $1.43\left(\mathrm{~m}, 1 \mathrm{H}\right.$, tetrahydropyran- $\mathrm{CH}_{2}$, minor diastereoisomer).

${ }^{13} \mathrm{C}$ NMR (101 MHz, Chloroform-d) $\delta 170.0,169.6,169.0,168.3,163.7,163.7,150.2,150.1$, $137.8,136.5,135.3,135.3,132.7,128.8,128.8,128.6,128.1,127.1,126.8,125.2,120.4$, 111.6, 111.4, 81.8, 81.7, 76.1, 57.0, 56.6, 53.4, 53.2, 53.0, 52.6, 30.7, 27.5, 26.8, 24.1, 12.8. ${ }^{8}$

IR 3730 (w), 2955 (w), 2361 (s), 2336 (s), 1744 (m), 1699 (s), 1666 (m), 1442 (w), 1275 (m), $1099(w), 976(w), 914(w), 736(m)$.

HRMS (ESI) calcd for $\mathrm{C}_{22} \mathrm{H}_{24} \mathrm{~N}_{2} \mathrm{NaO}_{7}{ }^{+}[\mathrm{M}+\mathrm{Na}]^{+}$451.1476; found 451.1474.

\section{Dimethyl (5-fluoro-2,4-dioxo-3,4-dihydropyrimidin-1(2H)-yl)-2-phenyldihydro-2H-pyran- $3,3(4 \mathrm{H})$-dicarboxylate (27).}
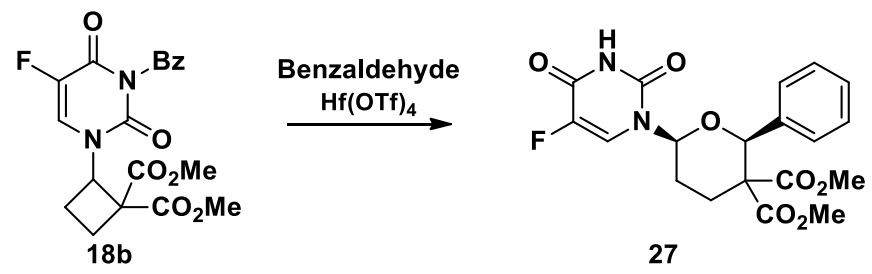

27

Following the general procedure, using benzaldehyde (46 $\mu \mathrm{L}, 0.45 \mathrm{mmol}, 1.5$ equiv) and cyclobutane 18b (121 mg, $0.300 \mathrm{mmol}, 1.0$ equiv), a mixture of diastereoisomers (13:1 by integration of methyl esters at $3.52 \mathrm{ppm}$ and $3.48 \mathrm{ppm}) 27$ (78 mg, $0.19 \mathrm{mmol}, 64 \% \mathrm{NMR}$ yield $)^{7}$ was obtained after column chromatography as a white foam. After recrystallization in ethanol, the pure product 27 (68 mg, $0.17 \mathrm{mmol}, 56 \%$ yield) was obtained as white foam and as a mixture of diastereoisomers ( $>20: 1$ by integration of methyl esters at $3.52 \mathrm{ppm}$ and 3.48 ppm).

RF (Pentane/Ethyl acetate $(1: 1))=0.32$.

MP $80.1-81.7^{\circ} \mathrm{C}$.

${ }^{1} \mathrm{H}$ NMR $(400 \mathrm{MHz}$, Chloroform-d, major diastereoisomer) $\delta 9.95(\mathrm{~s}, 1 \mathrm{H}, \mathrm{NH}), 7.63(\mathrm{~d}, J=5.8$ $\mathrm{Hz}, 1 \mathrm{H}, \mathrm{Ar}-\mathrm{H}), 7.39-7.34(\mathrm{~m}, 2 \mathrm{H}, \mathrm{Ar}-\mathrm{H}), 7.33-7.27(\mathrm{~m}, 3 \mathrm{H}, \mathrm{Ar}-\mathrm{H}$ and F-uracil $\mathrm{C}=\mathrm{C}-\mathrm{H}), 6.01$ - $5.93(\mathrm{~m}, 1 \mathrm{H}$, tetrahydropyran- $\mathrm{NCH}), 5.23(\mathrm{~s}, 1 \mathrm{H}$, tetrahydropyran- $\mathrm{CH}), 3.67(\mathrm{~s}, 3 \mathrm{H}$, ester $\left.\mathrm{CH}_{3}\right), 3.52\left(\mathrm{~s}, 3 \mathrm{H}\right.$, ester $\left.\mathrm{CH}_{3}\right), 2.71-2.62\left(\mathrm{~m}, 1 \mathrm{H}\right.$, tetrahydropyran- $\left.\mathrm{CH}_{2}\right), 2.40-2.19(\mathrm{~m}, 2 \mathrm{H}$, tetrahydropyran- $\left.\mathrm{CH}_{2}\right), 2.02-1.95\left(\mathrm{~m}, 1 \mathrm{H}\right.$, tetrahydropyran- $\left.\mathrm{CH}_{2}\right)$.

\footnotetext{
83 carbons of the minor diastereoisomer are not resolved.
} 
${ }^{13} \mathrm{C}$ NMR (101 MHz, Chloroform-d, major diastereoisomer) $\delta 170.1,169.1,156.98(\mathrm{~d}, J=26.6$ $\mathrm{Hz}$ ), 148.9, 140.93 (d, $J=238.5 \mathrm{~Hz}$ ), 137.2, 128.5, 127.7, 127.4, 124.12 (d, $J=33.6 \mathrm{~Hz}$ ), 82.2, 82.1, 56.9, 52.8, 52.1, 31.2, 26.5 .

IR 3204 (w), 3064 (w), 2955 (w), 2361 (m), 2257 (w), 1717 (s), 1457 (w), 1368 (w), 1265 (s), $1103(\mathrm{~m}), 1043(\mathrm{w}), 912(\mathrm{~s}), 731(\mathrm{~s})$.

HRMS (ESI) calcd for $\mathrm{C}_{19} \mathrm{H}_{19} \mathrm{FN}_{2} \mathrm{NaO}_{7}{ }^{+}[\mathrm{M}+\mathrm{Na}]^{+}$429.1068; found 429.1065.

6. Thymine based nucleoside analogues derivatizations.

\subsection{Acids.}

\section{General procedure for hydrolysis and decarboxylation reaction}

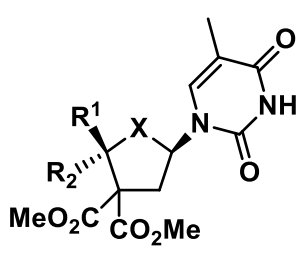

22b, 22e, 23b, 23c

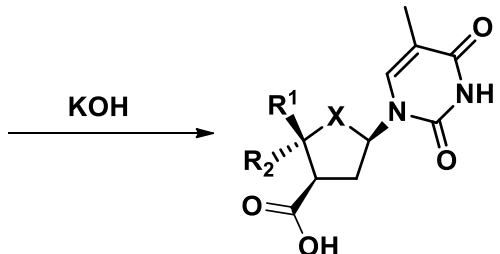

$54,55,29,56$

Compound 22b, 22e, 23b, and 23c (1 equiv) and $\mathrm{KOH}$ (4 equiv) were stirred under nitrogen in a dried and sealed microwave vial with dry methanol $(0.06 \mathrm{~mL})$ for 2 days at $70^{\circ} \mathrm{C}$. The thick yellow mixture was cooled down to room temperature and acidified with a $0.1 \mathrm{M} \mathrm{HCl}$ solution $(0.5 \mathrm{~mL})$. The mixture was extracted 3 times with AcOEt $(2 \mathrm{~mL})$, the organic layers were dried over anhydrous $\mathrm{MgSO}_{4}$ and concentrated under reduced pressure. The corresponding monoacid was obtained after column chromatography with a gradient of DCM to a solvent mixture of $\mathrm{DCM} / \mathrm{MeOH} 8: 2$ and $1 \% \mathrm{AcOH}$.

5-(5-Methyl-2,4-dioxo-3,4-dihydropyrimidin-1(2H)-yl)-2-phenyltetrahydrofuran-3carboxylic acid (54).<smiles>COC(=O)C1(C(C)=O)C[C@@H](n2cc(C)c(=O)[nH]c2=O)O[C@@H]1c1ccccc1</smiles>

22b

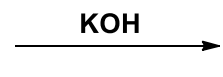

HO<smiles>Cc1cn([C@H]2C[C@H](C(=O)O)[C@@H](c3ccccc3)O2)c(=O)[nH]c1=O</smiles>

Following the general procedure for hydrolysis and decarboxylation reaction, using compound 22b $(0.050 \mathrm{~g}, 0.13 \mathrm{mmol}, 1$ equiv) and $\mathrm{KOH}(29 \mathrm{mg}, 0.52 \mathrm{mmol}, 4$ equiv) in dry $\mathrm{MeOH}(0.3$ $\mathrm{mL})$, the pure monoacid $54(0.034 \mathrm{~g}, 0.10 \mathrm{mmol}, 83 \%$ yield) was obtained as a white solid.

$\mathbf{R F}($ AcOEt $)=0.23$.

MP $123.2-125.3^{\circ} \mathrm{C}$.

${ }^{1} \mathrm{H}$ NMR $\left(400 \mathrm{MHz}\right.$, Methanol- $\left.d_{4}\right) \delta 7.37(\mathrm{~d}, J=8.6 \mathrm{~Hz}, 3 \mathrm{H}, \mathrm{Ar}-\mathrm{H}$ and thymine vinyl-CH), 7.32 - $7.22(\mathrm{~m}, 3 \mathrm{H}, \mathrm{Ar}-\mathrm{H}), 6.20-6.08(\mathrm{~m}, 1 \mathrm{H}$, tetrahydrofuran- $\mathrm{NCH}), 5.01(\mathrm{~d}, J=8.7 \mathrm{~Hz}, 1 \mathrm{H}$, tetrahydrofuran- $\mathrm{CH}), 3.35-3.24(\mathrm{~m}, 1 \mathrm{H}$, tetrahydrofuran- $\mathrm{CH}), 2.68(\mathrm{dt}, J=13.1,8.1 \mathrm{~Hz}, 1 \mathrm{H}$ tetrahydrofuran- $\left.\mathrm{CH}_{2}\right), 2.47$ (ddd, $J=13.8,9.5,4.5 \mathrm{~Hz}, 1 \mathrm{H}$, tetrahydrofuran- $\left.\mathrm{CH}_{2}\right), 1.78(\mathrm{~s}, 3 \mathrm{H}$, thymine methyl). 
${ }^{13} \mathrm{C}$ NMR $\left(101 \mathrm{MHz}\right.$, Methanol- $\left.d_{4}\right) \delta 174.0,165.0,150.9,138.9,137.2,128.2,126.2,110.4$, 85.7, 83.7, 49.9, 35.3, 11.0 .

The acid carbon is not defined.

IR 3429 (w), 3211 (w), 3039 (w), 2529 (w), 1695 (s), 1475 (w), $1272(\mathrm{~m}), 1068(\mathrm{~m}), 769(\mathrm{w})$, $701(\mathrm{~m})$.

HRMS (ESI) calcd for $\mathrm{C}_{16} \mathrm{H}_{16} \mathrm{~N}_{2} \mathrm{NaO}_{5}{ }^{+}[\mathrm{M}+\mathrm{Na}]^{+} 339.0951$; found 339.0944.

\section{5-(5-methyl-2,4-dioxo-3,4-dihydropyrimidin-1(2H)-yl)-2-((E)-styryl)tetrahydrofuran-3- carboxylic acid (55).}

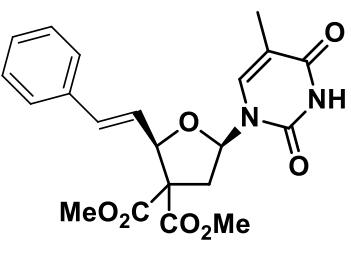

22e
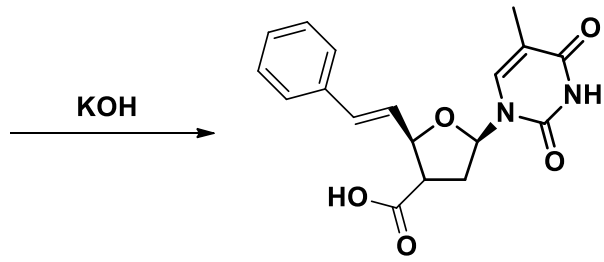

55

Following the general procedure for hydrolysis and decarboxylation reaction, using compound 22e $(0.14 \mathrm{~g}, 0.34 \mathrm{mmol}, 1$ equiv) and $\mathrm{KOH}(0.080 \mathrm{~g}, 1.4 \mathrm{mmol}, 4.0$ equiv) in dry $\mathrm{MeOH}$ (1.4 $\mathrm{mL}$ ). A mixture of unseparable monoacids (ratio 5:1 obtained by integration of the proton at $5.17 \mathrm{ppm}$ and $4.76 \mathrm{ppm}) 55$ (82 $\mathrm{mg}, 0.24 \mathrm{mmol}, 71 \%$ yield) was obtained as a white solid.

RF diacid $(\mathrm{DCM} / \mathrm{MeOH}(8: 2) 1 \% \mathrm{AcOH})=0.05$.

RF $56(\mathrm{DCM} / \mathrm{MeOH}(8: 2) 1 \% \mathrm{AcOH})=0.2$.

${ }^{1} \mathrm{H}$ NMR $\left(400 \mathrm{MHz}\right.$, Methanol- $\left.d_{4}\right) \delta 7.65(\mathrm{~s}, 1 \mathrm{H}$, thymine vinyl- $\mathrm{CH}$, minor diastereoisomer), 7.55 (s, $1 \mathrm{H}$, thymine vinyl- $\mathrm{CH}$, major diastereoisomer), $7.49(\mathrm{~d}, \mathrm{~J}=7.8 \mathrm{~Hz}, 4 \mathrm{H}, \mathrm{Ar}-\mathrm{H}$, major and minor diastereoisomers), $7.40-7.25$ ( $\mathrm{m}, 6 \mathrm{H}, \mathrm{Ar}-\mathrm{H}$, major and minor diastereoisomers), 6.76 (d, $J=15.9 \mathrm{~Hz}, 2 \mathrm{H}$, vinyl- $\mathrm{CH}$, major and minor diastereoisomers), 6.48 (ddd, $J=16.1,7.2,1.7$ $\mathrm{Hz}, 1 \mathrm{H}$, vinyl- $\mathrm{CH}$, major diastereoisomer), 6.34 (ddd, $J=16.0,6.7,1.7 \mathrm{~Hz}, 1 \mathrm{H}$, vinyl-CH, minor diastereoisomers), $6.26-6.17(\mathrm{~m}, 2 \mathrm{H}$, tetrahydrofuran- $\mathrm{NCH}$, major and minor diastereoisomers), 5.17 (t, $J=7.0 \mathrm{~Hz}, 1 \mathrm{H}$, tetrahydrofuran- $\mathrm{CH}$, minor diastereoisomer), 4.76 (t, $J=7.7 \mathrm{~Hz}, 1 \mathrm{H}$, tetrahydrofuran- $\mathrm{CH}$, major diastereoisomer), $3.25(\mathrm{q}, J=8.9 \mathrm{~Hz}, 1 \mathrm{H}$, tetrahydrofuran- $\mathrm{CH}$, major diastereoisomer), 3.18 (q, $J=8.9 \mathrm{~Hz}$, , tetrahydrofuran- $\mathrm{CH}$, major diastereoisomer), $2.86-2.71\left(\mathrm{~m}, 2 \mathrm{H}, \quad\right.$, tetrahydrofuran- $\mathrm{CH}_{2}$, major and minor diastereoisomers), 2.47 ( $\mathrm{m}, 2 \mathrm{H}$, tetrahydrofuran- $\mathrm{CH}_{2}$, major and minor diastereoisomers), 1.94 $(\mathrm{s}, 3 \mathrm{H}$, thymine methyl, minor diastereoisomer), $1.92(\mathrm{~s}, 3 \mathrm{H}$, thymine methyl, major diastereoisomer).

${ }^{13} \mathrm{C}$ NMR $\left(101 \mathrm{MHz}\right.$, Methanol- $\left.d_{4}\right) \delta 175.1,175.0,166.5,166.4,152.4,152.2,138.1,137.8$, 137.7, 137.6, 134.7, 133.6, 129.7, 129.7, 129.6, 129.2, 129.0, 128.5, 127.8, 127.7, 111.7, 111.5, 87.7, 86.9, 84.9, 84.8, 49.6, 49.4, 49.2, 49.0, 48.8, 48.6, 48.4, 36.7, 36.5, 12.5 .

For the major diastereoisomer, the acid carbon is not define and an aromatic one neither.

One carbon is missing for the minor diastereoisomer.

IR $3442(w), 3184(w), 3031(w), 2531(w), 1684(s), 1485(w), 1407(w), 1369(w), 1271(m)$, $1204(w), 1115(w), 1072(m), 980(w), 751(w), 695(m)$.

HRMS (ESI) calcd for $\mathrm{C}_{18} \mathrm{H}_{19} \mathrm{~N}_{2} \mathrm{O}_{5}{ }^{+}[\mathrm{M}+\mathrm{H}]^{+}$343.1288; found 343.1294.

.2-Methyl-5-(5-methyl-2,4-dioxo-3,4-dihydropyrimidin-1(2H)-yl)-2phenyltetrahydrofuran-3-carboxylic acid (29). 
<smiles>COC(C)(OC)C1(C)C[C@H](n2cc(C)c(=O)[nH]c2=O)O[C@@]1(C)c1ccccc1</smiles>

23b<smiles>Cc1cn([C@H]2C[C@H](C(=O)O)[C@@](C)(c3ccccc3)O2)c(=O)[nH]c1=O</smiles>

29

Following the general procedure for hydrolysis and decarboxylation reaction, using compound 23b $(0.020 \mathrm{~g}, 0.050 \mathrm{mmol}, 1$ equiv) and $\mathrm{KOH}(6 \mathrm{mg}, 0.1 \mathrm{mmol}, 4$ equiv), the pure monoacid $29(0.014 \mathrm{~g}, 0.042 \mathrm{mmol}, 83 \%$ yield $)$ was obtained as a white solid. The stereochemistry of the acid was determined by NOE experiment.

$\mathbf{R F}(\mathrm{AcOEt})=0.18$

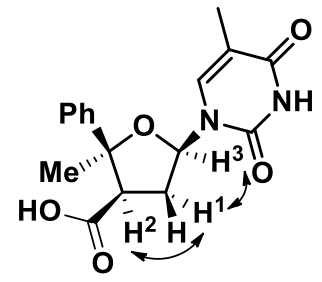

MP 236.2-236. ${ }^{\circ} \mathrm{C}$.

${ }^{1} \mathrm{H}$ NMR $\left(400 \mathrm{MHz}\right.$, Acetone- $\left.d_{6}\right) \delta 11.39(\mathrm{br} \mathrm{s}, 1 \mathrm{H}, \mathrm{COOH}), 10.01(\mathrm{~s}, 1 \mathrm{H}$, thymine $\mathrm{NH}), 7.70-$ $7.62(\mathrm{~m}, 2 \mathrm{H}, \mathrm{Ar}-\mathrm{H}), 7.48-7.28(\mathrm{~m}, 3 \mathrm{H}, \mathrm{Ar}-\mathrm{H}), 7.25(\mathrm{q}, \mathrm{J}=1.1 \mathrm{~Hz}, 1 \mathrm{H}$, thymine vinyl-CH), 6.46 (dd, $J=7.5,4.2 \mathrm{~Hz}, 1 \mathrm{H}$, tetrahydrofuran- $\mathrm{NCH}$ ), $3.75(\mathrm{t}, J=8.7 \mathrm{~Hz}, 1 \mathrm{H}$, tetrahydrofuran- $\mathrm{CH}$ ), 2.99 (ddd, $J=14.1,8.9,7.6 \mathrm{~Hz}, 1 \mathrm{H}$, tetrahydrofuran- $\mathrm{CH}_{2}$ ), 2.51 (ddd, $J=14.0,8.7,4.2 \mathrm{~Hz}, 1 \mathrm{H}$, tetrahydrofuran- $\left.\mathrm{CH}_{2}\right), 1.71(\mathrm{~d}, J=1.3 \mathrm{~Hz}, 3 \mathrm{H}$, thymine methyl), $1.60(\mathrm{~s}, 3 \mathrm{H}$, tetrahydrofuran methyl).

${ }^{13} \mathrm{C}$ NMR $\left(101 \mathrm{MHz}\right.$, Acetone- $\left.d_{6}\right) \delta 177.8,169.4,156.0,150.9,142.1,133.4,132.6,130.5$, $115.2,92.0,90.0,57.2,39.5,30.0,16.7$.

IR 3220 (w), $3054(w), 2925$ (w), $2854(w), 1704(s), 1660(m), 1478(w), 1419(w), 1271(w)$, $1110(w), 1058(w), 855(w), 800(w), 769(w)$.

HRMS (ESI) calcd for $\mathrm{C}_{17} \mathrm{H}_{19} \mathrm{~N}_{2} \mathrm{O}_{5}{ }^{+}[\mathrm{M}+\mathrm{H}]^{+} 331.1288$; found 331.1281.

\section{2-(4-Fluorophenyl)-2-methyl-5-(5-methyl-2,4-dioxo-3,4-dihydropyrimidin-1(2H)-} yl)tetrahydrofuran-3-carboxylic acid (56).<smiles>CC(=O)C1(C(C)=O)CC(n2cc(C)c(=O)[nH]c2=O)O[C@]1(C)c1ccc(F)cc1</smiles>

23c

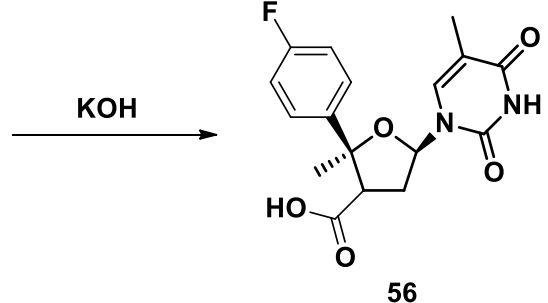

Following the general procedure for hydrolysis and decarboxylation reaction, using compound 23c $(0.040 \mathrm{~g}, 0.095 \mathrm{mmol}, 1$ equiv) and $\mathrm{KOH}(21 \mathrm{mg}, 0.38 \mathrm{mmol}, 4$ equiv) in a mixture of $\mathrm{MeOH} /$ water $(0.1 \mathrm{~mL} / 0.1 \mathrm{~mL})$, a mixture of unseparable monoacids (ratio 10:1 obtained by integration of the proton at $6.50 \mathrm{ppm}$ and $6.40 \mathrm{ppm}) 56(0.028 \mathrm{~g}, 0.080 \mathrm{mmol}, 84 \%$ yield, ) were obtained as a white solid.

$\mathbf{R F}(\mathrm{DCM} / \mathrm{MeOH}(9: 1))=0.15$.

MP $193.4-194.2^{\circ} \mathrm{C}$. 
${ }^{1} \mathrm{H}$ NMR $\left(400 \mathrm{MHz}\right.$, Acetone- $\left.d_{6}\right) \delta 10.08$ (br.s, $\left.1 \mathrm{H}, \mathrm{COOH}\right), 8.17$ (s, $\left.1 \mathrm{H}, \mathrm{NH}\right), 7.68$ (dd, $J=8.1$, $4.9 \mathrm{~Hz}, 2 \mathrm{H}, \mathrm{Ar}-\mathrm{H}$, major diastereoisomer), $7.55(\mathrm{dd}, \mathrm{J}=8.0,4.8 \mathrm{~Hz}, 2 \mathrm{H}, \mathrm{Ar}-\mathrm{H}$, minor diastereoisomer), $7.30(\mathrm{~s}, 1 \mathrm{H}$, thymine vinyl- $\mathrm{CH}$, major diastereoisomer), $7.13(\mathrm{t}, \mathrm{J}=8.3 \mathrm{~Hz}$, $2 \mathrm{H}, \mathrm{Ar}-\mathrm{H}$, major diastereoisomer), 7.07 (t, $J=8.4 \mathrm{~Hz}, 2 \mathrm{H}$, , Ar- $\mathrm{H}$, minor diastereoisomer), 6.50 (t, $J=7.0 \mathrm{~Hz}, 1 \mathrm{H}$, , tetrahydrofuran- $\mathrm{NCH}$, minor diastereoisomer), $6.40(\mathrm{dd}, J=8.0,3.9 \mathrm{~Hz}$, $1 \mathrm{H}$, tetrahydrofuran- $\mathrm{NCH}$, major diastereoisomer), 3.72 (t, $J=9.1 \mathrm{~Hz}, 1 \mathrm{H}$, tetrahydrofuran- $\mathrm{CH}$, major diastereoisomer), $3.46(\mathrm{dd}, J=9.2,4.1 \mathrm{~Hz}, 1 \mathrm{H}$, tetrahydrofuran- $\mathrm{CH}$, minor diastereoisomer), 3.00 (dt, $J=13.4,8.7 \mathrm{~Hz}, 1 \mathrm{H}$, tetrahydrofuran- $\mathrm{CH}_{2}$, major diastereoisomer), 2.93 (dd, $J=15.4,7.9 \mathrm{~Hz}, 1 \mathrm{H}$, tetrahydrofuran- $\mathrm{CH}_{2}$, minor diastereoisomer), 2.53 (ddd, $J=$ 13.7, 8.9, $3.9 \mathrm{~Hz}, 1 \mathrm{H}$, tetrahydrofuran- $\mathrm{CH}_{2}$, major diastereoisomer $), 2.46-2.35(\mathrm{~m}, 1 \mathrm{H}$, tetrahydrofuran- $\mathrm{CH}_{2}$, minor diastereoisomer), $1.80(\mathrm{~s}, 3 \mathrm{H}$, thymine methyl, minor diastereoisomer), $1.74(\mathrm{~s}, 3 \mathrm{H}$, thymine methyl, major diastereoisomer).

${ }^{13} \mathrm{C}$ NMR (101 MHz, Acetone- $\left.d_{6}\right) \delta 174.7,164.3,164.2,164.0,162.8(\mathrm{~d}, J=244.1 \mathrm{~Hz}), 162.7$ (d, $J=244.1 \mathrm{~Hz}) .151 .4,151.3,142.7(\mathrm{~d}, J=3.0 \mathrm{~Hz}), 139.6(\mathrm{~d}, J=2.9 \mathrm{~Hz}), 137.8,137.5,128.5$ (d, $J=8.1 \mathrm{~Hz}), 128.4(\mathrm{~d}, J=8.2 \mathrm{~Hz}), 115.5(\mathrm{~d}, J=21.4 \mathrm{~Hz}), 115.1(\mathrm{~d}, J=21.4 \mathrm{~Hz}), 111.0$, $110.9,86.9,86.9,85.5,83.2,53.9,53.2,35.2,34.9,12.7,12.3$.

IR 3530 (w), $3189(w), 3065$ (w), 2927 (w), $1697(s), 1512(m), 1268(m), 1088(w), 1052(w)$, $839(\mathrm{~m})$.

HRMS (ESI) calcd for $\mathrm{C}_{17} \mathrm{FH}_{18} \mathrm{~N}_{2} \mathrm{O}_{5}{ }^{+}[\mathrm{M}+\mathrm{H}]^{+} 349.1194$; found 349.1194.

\subsection{Alcohols.}

\section{General procedure for reduction of carboxylic acids.}

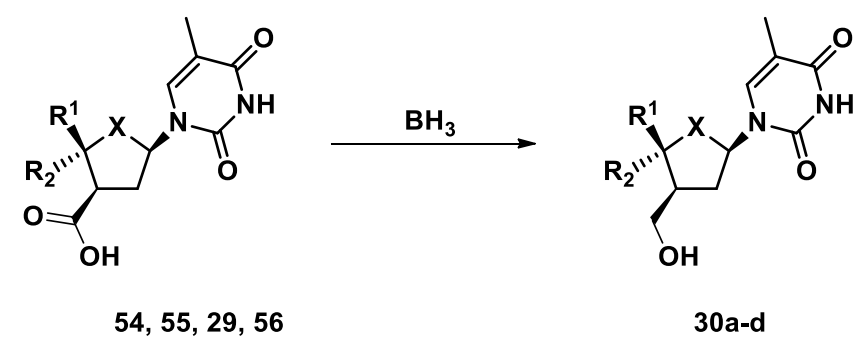

The carboxylic acid (1 equiv) was solubilized in dry THF in a dried round bottom flask under nitrogen. The reaction mixture under nitrogen was cooled to $0{ }^{\circ} \mathrm{C}$ and dimethylsulfide borane solution ( $2 \mathrm{M}$ in THF, $0.042 \mathrm{~mL}, 0.083 \mathrm{mmol}, 2.2$ equiv) was added dropwise. The reaction was allowed to slowly warm up and was stirred under nitrogen for $16 \mathrm{~h}$. The reaction mixture was quenched by addition of a saturated solution of $\mathrm{Na}_{2} \mathrm{CO}_{3}(0.5 \mathrm{~mL})$ and acidified by addition of a $1 \mathrm{M} \mathrm{HCl}$ solution $(1 \mathrm{~mL})$. Then the mixture was extracted three times with AcOEt $(3 \mathrm{~mL})$ and the organic layers were dried over anhydrous $\mathrm{MgSO}_{4}$. The crude product was purified by column chromatography with a gradient of pure AcOEt to a mixture of $\mathrm{AcOEt} / \mathrm{MeOH}(8: 2)$, affording the pure alcohol, as a colorless foam.

4-(Hydroxymethyl)-5-phenyltetrahydrofuran-2-yl)-5-methylpyrimidine-2,4(1H,3H)-dione (30a). 


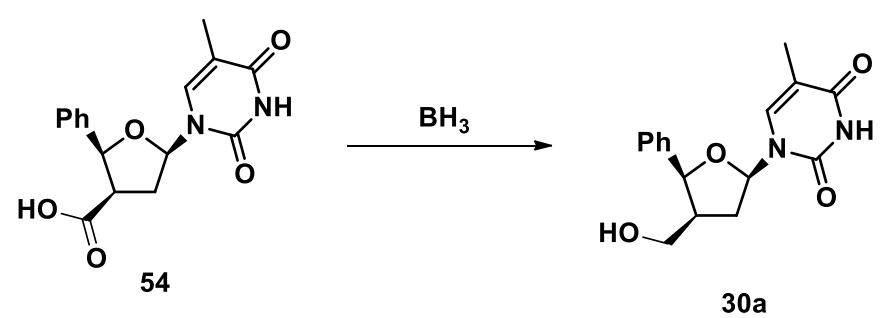

Following the general procedure for reduction of carboxylic acids, using compound $\mathbf{5 4}(0.012$ g, $0.038 \mathrm{mmol}, 1$ equiv) and DMS solution $(0.042 \mathrm{~mL}, 0.083 \mathrm{mmol}, 2.2$ equiv) in dry THF (0.35 $\mathrm{mL})$, the alcohol $30 \mathrm{a}(0.0095 \mathrm{~g}, 0.031 \mathrm{mmol}, 86 \%$ yield, $71 \%$ over the two steps) was obtained as a colorless oil.

$\mathbf{R F}(\mathrm{AcOEt})=0.34$.

MP $68.1-69.4^{\circ} \mathrm{C}$.

${ }^{1} \mathrm{H}$ NMR $\left(400 \mathrm{MHz}\right.$, Methanol- $\left.d_{4}\right) \delta 7.53-7.47(\mathrm{~m}, 1 \mathrm{H}$, thymine vinyl- $\mathrm{CH}), 7.49-7.28(\mathrm{~m}, 5 \mathrm{H}$, $\mathrm{Ar}-\mathrm{H}), 6.29-6.09(\mathrm{~m}, 1 \mathrm{H}$, tetrahydrofuran- $\mathrm{NCH}), 4.78(\mathrm{~d}, J=8.6 \mathrm{~Hz}, 1 \mathrm{H}$, tetrahydrofuran$\mathrm{CH}$ ), 3.68 (dd, $J=11.2,4.4 \mathrm{~Hz}, 1 \mathrm{H},-\mathrm{CH}_{2} \mathrm{OH}$ ), $3.64-3.53$ (dd, $J=11.2,5.6 \mathrm{~Hz}, 1 \mathrm{H},-\mathrm{CH}_{2} \mathrm{OH}$ ), 2.61 (ddd, $J=14.2,8.3,2.8 \mathrm{~Hz}, 1 \mathrm{H}$, tetrahydrofuran- $\mathrm{CH}$ ), 2.49 (ddd, $J=13.7,8.7,7.6 \mathrm{~Hz}, 1 \mathrm{H}$, tetrahydrofuran- $\mathrm{CH}_{2}$ ), 2.35 (ddd, $J=13.6,9.0,4.5 \mathrm{~Hz}, 1 \mathrm{H}$, tetrahydrofuran- $\left.\mathrm{CH}_{2}\right), 1.88(\mathrm{~d}, J=$ $2.4 \mathrm{~Hz}, 3 \mathrm{H}$, thymine methyl).

${ }^{13} \mathrm{C}$ NMR $\left(101 \mathrm{MHz}\right.$, Methanol- $\left.d_{4}\right) \delta 166.5,152.6,141.4,138.4,129.85,129.5,128.1,111.9$, 86.7, 84.8, 62.3, 49.1, 35.9, 12.7.

IR 3410 (w), 3207 (w), 2927 (w), 2520 (w), $1686(\mathrm{~s}), 1471(\mathrm{~m}), 1271(\mathrm{~m}), 1055(\mathrm{~m}), 911(\mathrm{w})$, $760(\mathrm{~m})$.

HRMS (ESI) calcd for $\mathrm{C}_{16} \mathrm{H}_{18} \mathrm{~N}_{2} \mathrm{NaO}_{4}{ }^{+}[\mathrm{M}+\mathrm{Na}]^{+}$325.1159; found 325.1159.

\section{4-(Hydroxymethyl)-5-((E)-styryl)tetrahydrofuran-2-yl)-5-methylpyrimidine-2,4(1 H,3H)- dione (30b).}

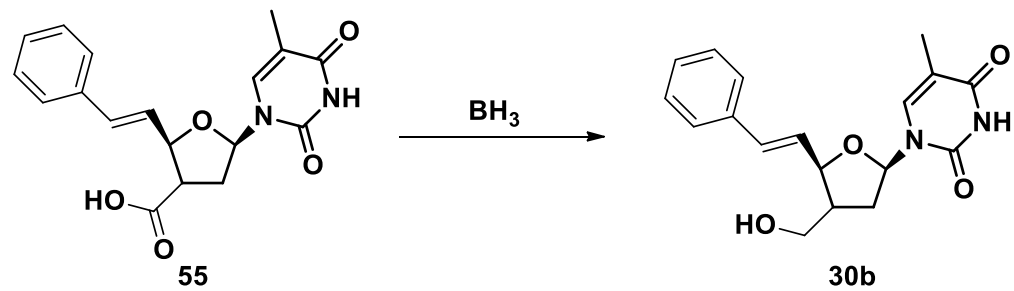

Following the general procedure for reduction of carboxylic acids, using the mixture of diastereoisomers $55(0.020 \mathrm{~g}, 0.058 \mathrm{mmol}, 1$ equiv) and DMS solution $(0.063 \mathrm{~mL}, 0.13 \mathrm{mmol}$, 2.2 equiv) in dry THF $(0.53 \mathrm{~mL})$, the mixture of diastereoisomeric alcohols (ratio $5: 1$ obtained by integration of the proton at $4.42 \mathrm{ppm}$ and $4.65 \mathrm{ppm}) 30 \mathrm{~b}(0.016 \mathrm{~g}, 0.049 \mathrm{mmol}, 83 \%$ yield, $56 \%$ over the two steps) was obtained as colorless oil.

RF $(\mathrm{DCM} / \mathrm{MeOH}(9.5: 0.5))=0.25$.

${ }^{1} \mathrm{H}$ NMR (400 MHz, Chloroform-d, major diastereoisomer) $\delta 8.24$ (br.s, $1 \mathrm{H}$, thymine-NH), 7.40 $-7.20(\mathrm{~m}, 6 \mathrm{H}, \mathrm{Ar}-\mathrm{H}$ and thymine vinyl-CH), $6.67(\mathrm{~d}, J=16.0 \mathrm{~Hz}, 1 \mathrm{H}$, vinyl-CH), $6.24(\mathrm{dd}, J=$ $15.8,7.1 \mathrm{~Hz}, 1 \mathrm{H}$, vinyl-CH), 6.07 (dd, $J=6.7,3.6 \mathrm{~Hz}, 1 \mathrm{H}$, tetrahydrofuran- $\mathrm{NCH}), 4.42(\mathrm{t}, J=$ $7.5 \mathrm{~Hz}, 1 \mathrm{H}$, tetrahydrofuran- $\mathrm{CH}), 3.74\left(\mathrm{dd}, J=10.7,5.0 \mathrm{~Hz}, 1 \mathrm{H},-\mathrm{CH}_{2} \mathrm{OH}\right), 3.68(\mathrm{dd}, J=10.7$, $5.1 \mathrm{~Hz}, 1 \mathrm{H},-\mathrm{CH}_{2} \mathrm{OH}$ ), $2.46-2.26\left(\mathrm{~m}, 2 \mathrm{H}\right.$, tetrahydrofuran- $\mathrm{CH}_{2}$ ), 2.16 (ddd, $J=12.1,7.1,3.5$ $\mathrm{Hz}, 1 \mathrm{H}$, tetrahydrofuran- $\mathrm{CH}), 1.86(\mathrm{~d}, J=1.2 \mathrm{~Hz}, 3 \mathrm{H}$, thymine methyl). 
${ }^{13} \mathrm{C}$ NMR (101 MHz, Chloroform- $d$, major diastereoisomer) $\delta 164.1,150.6,136.4,136.0,134.5$, $129.3,129.0,127.4,127.3,111.2,86.2,84.1,62.7,46.1,36.3,13.3$.

IR 2962 (w), 2924 (w), 2853 (w), 1687 (s), $1471(w), 1363$ (w), 1268 (m), 1189 (w), 1055 (s), $967(\mathrm{~m}), 744(\mathrm{~m})$.

HRMS (ESI) calcd for $\mathrm{C}_{18} \mathrm{H}_{20} \mathrm{~N}_{2} \mathrm{NaO}_{4}{ }^{+}[\mathrm{M}+\mathrm{Na}]^{+} 351.1315$; found 351.1319.

\section{4-(Hydroxymethyl)-5-methyl-5-phenyltetrahydrofuran-2-yl)-5-methylpyrimidine-} 2,4(1H,3H)-dione (30c).
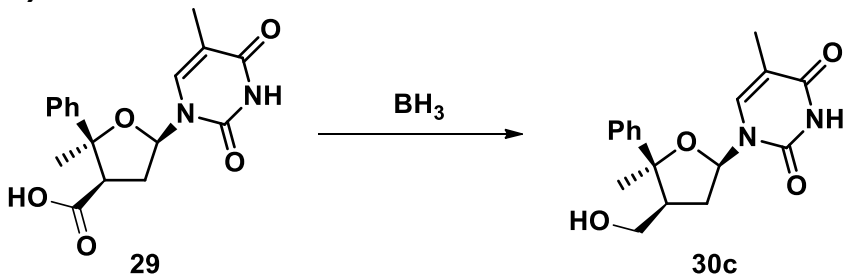

Following the general procedure for reduction of carboxylic acids, using compound $29(0.018$ $\mathrm{g}, 0.054 \mathrm{mmol}, 1$ equiv) and DMS solution $(0.060 \mathrm{~mL}, 0.12 \mathrm{mmol}, 2.2$ equiv) in dry THF (0.5 $\mathrm{mL})$, the alcohol $30 \mathrm{c}(0.013 \mathrm{~g}, 0.041 \mathrm{mmol}, 75 \%$ yield, $63 \%$ over the two steps) was obtained as a colorless foam.

$\mathbf{R F}($ AcOEt $)=0.13$

MP $79.4-83.4^{\circ} \mathrm{C}$.

${ }^{1} \mathrm{H}$ NMR $(400 \mathrm{MHz}$, Chloroform- $d) \delta 8.60(\mathrm{~s}, 1 \mathrm{H}$, thymine-NH), $7.46(\mathrm{~d}, J=7.6 \mathrm{~Hz}, 2 \mathrm{H}, \mathrm{Ar}-\mathrm{H})$, $7.39-7.22(\mathrm{~m}, 3 \mathrm{H}, \operatorname{Ar}-\mathrm{H}), 6.82(\mathrm{~s}, 1 \mathrm{H}$, thymine vinyl-CH$), 6.21(\mathrm{~d}, J=3.6 \mathrm{~Hz}, 1 \mathrm{H}$, tetrahydrofuran- $\mathrm{NCH}$ ), $3.96\left(\mathrm{dd}, J=10.5,5.4 \mathrm{~Hz}, 1 \mathrm{H},-\mathrm{CH}_{2} \mathrm{OH}\right), 3.74(\mathrm{dd}, J=10.7,7.9 \mathrm{~Hz}, 1 \mathrm{H}$, $\left.-\mathrm{CH}_{2} \mathrm{OH}\right), 2.75(\mathrm{dd}, \mathrm{J}=11.0,4.6 \mathrm{~Hz}, 1 \mathrm{H}$, tetrahydrofuran- $\mathrm{CH}), 2.44-2.25(\mathrm{~m}, 2 \mathrm{H}$, tetrahydrofuran- $\mathrm{CH}_{2}$ ), 2.04 (br.s, $\left.1 \mathrm{H}, \mathrm{OH}\right), 1.60$ (s, 3H, thymine methyl), $1.42(\mathrm{~s}, 3 \mathrm{H}$, tetrahydrofuran methyl).

${ }^{13} \mathrm{C}$ NMR (101 MHz, Chloroform- $\left.d\right) \delta 163.7,150.4,145.6,136.3,128.6,127.7,125.1,110.3$, 87.6, 85.0, 62.6, 47.5, 36.6, 24.5, 12.5.

IR 3446 (w), 2959 (w), 1679 (s), 1473 (w), 1448 (w), 1265 (m), 1031 (s), 911 (w), 767 (m), 735 (m).

HRMS (ESI) calcd for $\mathrm{C}_{17} \mathrm{H}_{20} \mathrm{~N}_{2} \mathrm{NaO}_{4}{ }^{+}[\mathrm{M}+\mathrm{Na}]^{+} 339.1315$; found 339.1317.

5-(4-Fluorophenyl)-4-(hydroxymethyl)-5-methyltetrahydrofuran-2-yl)-5methylpyrimidine-2,4(1 H,3H)-dione (30d).

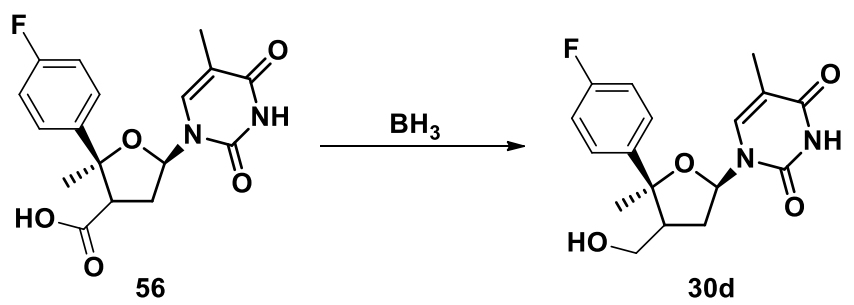

Following the general procedure for reduction of carboxylic acids, using the mixture of diastereoisomers $56(0.027 \mathrm{~g}, 0.078 \mathrm{mmol}, 1$ equiv) and DMS solution $(0.085 \mathrm{~mL}, 0.17 \mathrm{mmol}$, 2.2 equiv) in dry THF $(0.5 \mathrm{~mL})$, the mixture of diastereoisomeric alcohols (ratio 10:1 obtained 
by integration of the proton at $3.31 \mathrm{ppm}$ and $3.91 \mathrm{ppm}) 30 \mathrm{~d}(0.020 \mathrm{~g}, 0.059 \mathrm{mmol}, 76 \%$ yield, $64 \%$ over the two steps) was obtained as colorless oil.

$\mathbf{R F}(\mathrm{AcOE} t)=0.53$

MP $192.3-193.8^{\circ} \mathrm{C}$ (decomp.).

${ }^{1} \mathrm{H}$ NMR (400 MHz, Chloroform- $\left.d\right) \delta 8.40$ (s, $1 \mathrm{H}$, thymine- $\mathrm{NH}$, minor and major diastereoisomers), $7.58(\mathrm{~d}, J=1.3 \mathrm{~Hz}, 1 \mathrm{H}$, thymine vinyl- $\mathrm{CH}$, minor diastereoisomer), 7.46 (dd, $J=8.9,5.2 \mathrm{~Hz}, 2 \mathrm{H}, \mathrm{Ar}-\mathrm{H}$, major diastereoisomer), 7.33 (dd, $J=8.9,5.2 \mathrm{~Hz}, 2 \mathrm{H}, \mathrm{Ar}-\mathrm{H}$, minor diastereoisomer), $7.01(\mathrm{t}, J=8.7 \mathrm{~Hz}, 4 \mathrm{H}, \mathrm{Ar}-\mathrm{H}$, minor and major diastereoisomers), $6.82(\mathrm{~d}, J$ $=1.3 \mathrm{~Hz}, 1 \mathrm{H}$, thymine vinyl-CH, major diastereoisomer $), 6.23(\mathrm{~d}, J=7.0 \mathrm{~Hz}, 1 \mathrm{H}$ tetrahydrofuran- $\mathrm{NCH}$, minor diastereoisomer), 6.19 (dd, $J=6.9,3.4 \mathrm{~Hz}, 1 \mathrm{H}$ tetrahydrofuran$\mathrm{NCH}$, major diastereoisomer), 3.91 (dd, $J=10.6,6.0 \mathrm{~Hz}, 1 \mathrm{H},-\mathrm{CH}_{2} \mathrm{OH}$, major diastereoisomer), 3.75 (dd, $J=10.6,7.5 \mathrm{~Hz}, 1 \mathrm{H},-\mathrm{CH}_{2} \mathrm{OH}$, major diastereoisomer), 3.31 (dd, $J=11.2,5.1 \mathrm{~Hz}$, $\mathrm{OH},-\mathrm{CH}_{2} \mathrm{OH}$, minor diastereoisomer), 3.23 (dd, $J=11.2,6.0 \mathrm{~Hz}, \mathrm{OH},-\mathrm{CH}_{2} \mathrm{OH}$, minor diastereoisomer), $2.72(\mathrm{~m}, 2 \mathrm{H}$, tetrahydrofuran- $\mathrm{CH}$, minor and major diastereoisomers), 2.45 - $2.22\left(\mathrm{~m}, 4 \mathrm{H}\right.$, tetrahydrofuran- $\mathrm{CH}_{2}$, minor and major diastereoisomers), $1.89(\mathrm{~d}, J=1.2 \mathrm{~Hz}$, $3 \mathrm{H}$, thymine methyl, minor diastereoisomer), 1.81 (br.s, $1 \mathrm{H}, \mathrm{OH}$, minor and major diastereoisomer), $1.66(\mathrm{~d}, J=1.2 \mathrm{~Hz}, 3 \mathrm{H}$, thymine methyl, major diastereoisomer), $1.57(\mathrm{~s}, 3 \mathrm{H}$, tetrahydrofuran methyl, minor diastereoisomer), $1.41(\mathrm{~s}, 3 \mathrm{H}$, tetrahydrofuran methyl, major diastereoisomer).

${ }^{13} \mathrm{C}$ NMR (101 MHz, Chloroform- $d$, Major diastereoisomer) $\delta 163.5,162.1$ (d, $J=247.3 \mathrm{~Hz}$ ), 150.3, 141.5 (d, $J=3.6 \mathrm{~Hz}), 136.0,127.0(\mathrm{~d}, J=8.0 \mathrm{~Hz}), 115.3(\mathrm{~d}, J=21.3 \mathrm{~Hz}), 110.5,87.3$, 84.9, 62.5, 47.8, 36.5, 24.3, 12.6 .

IR 2981 (w), 2925 (w), 2860 (w), 1680 (m), $1511(w), 1473$ (w), 1273 (w), $1230(w), 1055(m)$, $1013(\mathrm{~m}), 909(\mathrm{~s}), 733(\mathrm{~s})$.

HRMS (ESI) calcd for $\mathrm{C}_{17} \mathrm{H}_{19} \mathrm{FN}_{2} \mathrm{NaO}_{4}{ }^{+}[\mathrm{M}+\mathrm{Na}]^{+} 357.1221$; found 357.1231.

\subsection{Carbonucleoside alcohol.}

5-Methyl-2,4-dioxo-3,4-dihydropyrimidin-1(2H)-yl-2-phenylcyclopent-1-ene-1-carboxylic acid (31).<smiles>Cc1cn(C2CC(C(=O)O)(C(=O)O)[C@@](OC(=O)[O-])(c3ccccc3)C2)c(=O)[nH]c1=O</smiles>

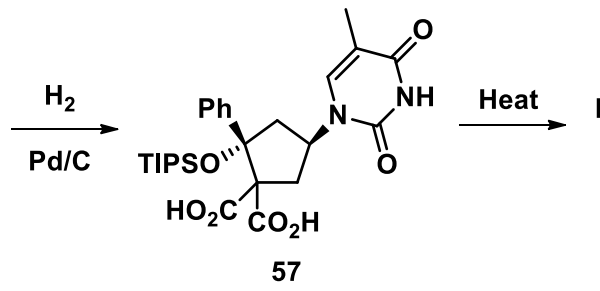<smiles>Cc1cn(C2CC(=O)C(=O)C2)c(=O)[nH]c1=O</smiles>

31

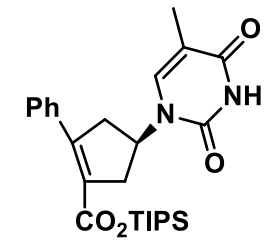

58

Compound 24c ( $0.10 \mathrm{~g}, 0.14 \mathrm{mmol}, 1.0$ equiv) and $\mathrm{Pd}-\mathrm{C}(0.030 \mathrm{~g}, 0.014 \mathrm{mmol}, 0.1$ equiv) were stirred in a flame-dried flask under $\mathrm{H}_{2}$ at $57^{\circ} \mathrm{C}$ with ethanol $(10 \mathrm{~mL}) 5 \mathrm{~min}$ to solubilize the starting material, then the reaction was let for $10 \mathrm{~min}$ to cool down. The reaction mixture was filtered on a pore 5 filter with hot ethanol $(50 \mathrm{~mL})$ to afford after solvent evaporation, the pure diacid 57 as colorless needles. Then the crude product was heated neat at $80{ }^{\circ} \mathrm{C}$ for $16 \mathrm{~h}$. After column chromatography using DCM to a mixture of $\mathrm{DCM} / \mathrm{MeOH}(9: 1)$ with $1 \% \mathrm{AcOH}$ as solvent, the pure product 31 (28 mg, $0.090 \mathrm{mmol}, 64 \%$ yield) was obtained as a colorless oil. The corresponding TIPS protected carboxylic acid 58 (14 mg, $0.030 \mathrm{mmol}, 20 \%$ yield) was also isolated as a colorless oil.

RF $57(\mathrm{DCM} / \mathrm{MeOH}(9: 1))=0.37$ 
RF $31(\mathrm{DCM} / \mathrm{MeOH}(9: 1))=0.21$.

MP $114.1-115.6^{\circ} \mathrm{C}$.

${ }^{1} \mathrm{H}$ NMR $(400 \mathrm{MHz}$, Chloroform- $d) \delta 9.20(\mathrm{~d}, J=9.1 \mathrm{~Hz}, 1 \mathrm{H}$, thymine-NH), 7.37 (s, 5H, Ar-H), $7.08(\mathrm{~d}, J=1.6 \mathrm{~Hz}, 1 \mathrm{H}$, , thymine vinyl- $\mathrm{CH}), 5.37$ (tt, $J=8.8,4.3 \mathrm{~Hz}, 1 \mathrm{H}$, cyclopentane- $\mathrm{NCH})$, $3.60-3.25\left(\mathrm{~m}, 2 \mathrm{H}\right.$, cyclopentane- $\left.\mathrm{CH}_{2}\right), 2.96$ (dddd, $J=18.9,8.2,3.7,1.9 \mathrm{~Hz}, 2 \mathrm{H}$, cyclopentane- $\left.\mathrm{CH}_{2}\right), 1.92(\mathrm{~d}, J=1.2 \mathrm{~Hz}, 3 \mathrm{H}$, thymine methyl).

${ }^{13} \mathrm{C}$ NMR (101 MHz, Chloroform- $\left.d\right) \delta$ 168.2, 163.7, 153.1, 150.5, 136.2, 134.4, 128.8, 128.0, 127.7, 125.7, 111.8, 46.2, 40.8, 12.5 .

IR 3169 (w), 3026 (w), 2929 (w), 1675 (s), $1472(w), 1393(w), 1270(m), 1221(w), 909(m)$, $735(\mathrm{~s}), 636(\mathrm{w})$.

HRMS (ESI) calcd for $\mathrm{C}_{17} \mathrm{H}_{15}\left[{ }^{2} \mathrm{H}_{2} \mathrm{~N}_{2} \mathrm{O}_{4}{ }^{+}[\mathrm{M}+\mathrm{H}]^{+} 315.1306\right.$; found 315.1300.

\section{5-Methyl-2,4-dioxo-3,4-dihydropyrimidin-1(2H)-yl-2-phenylcyclopentane-1-carboxylic} acid (59).<smiles>Cc1cn(C2CC(C(=O)O)=C(c3ccccc3)C2)c(=O)[nH]c1=O</smiles>

31<smiles>Cc1cn([C@H]2C[C@H](C(=O)O)[C@H](P)[C@H]2c2ccccc2)c(=O)[nH]c1=O</smiles>

59

Carboxylic acid 31 (0.021 g, 0.067 mmol, 1.0 equiv) and Pd-C (0.01 g, $0.007 \mathrm{mmol}, 5$ \% wt, 0.1 equiv) were stirred in a flame dried flask under $\mathrm{H}_{2}$ at room temperature with ethanol $(1 \mathrm{~mL})$. TLC shows that the reaction was accomplished after 10 minutes and filtered on pore 5 filter. The residue was washed several times with hot ethanol. The pure product $59(0.018 \mathrm{~g}, 0.057$ $\mathrm{mmol}, 85 \%$ yield) precipitated directly as white spheres. The stereochemistry was assigned by NOE experiment.

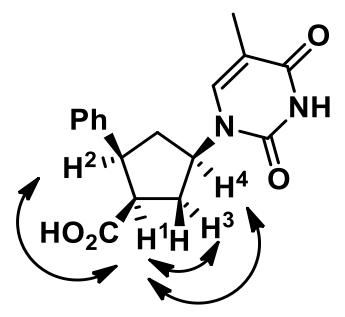

$\mathbf{R F}(\mathrm{DCM} / \mathrm{MeOH}(8: 2) 1 \% \mathrm{AcOH})=0.49$.

MP $227.8-236.9^{\circ} \mathrm{C}$.

${ }^{1} \mathbf{H}$ NMR (400 MHz, DMSO- $\left.d_{6}\right) \delta 11.86(\mathrm{~s}, 1 \mathrm{H},-\mathrm{COOH}), 11.20(\mathrm{~s}, 1 \mathrm{H},-\mathrm{NH}), 7.80$ (d, J = 1.4 $\mathrm{Hz}, 1 \mathrm{H}$, , thymine vinyl-CH), $7.29-7.10(\mathrm{~m}, 5 \mathrm{H}, \mathrm{Ar}-\mathrm{H}), 4.97(\mathrm{dtd}, J=11.3,9.1,6.8 \mathrm{~Hz}, 1 \mathrm{H}$, cyclopentane- $\mathrm{NCH}$ ), 3.48 (ddd, $J=12.3,9.1,6.2 \mathrm{~Hz}, 1 \mathrm{H}$, cyclopentane-CH), $3.19-3.00(\mathrm{~m}$, $1 \mathrm{H}), 2.36-2.21(\mathrm{~m}, 2 \mathrm{H}$, cyclopentane-H), $2.11(\mathrm{dt}, J=12.3,6.5 \mathrm{~Hz}, 1 \mathrm{H}$, cyclopentane- $\mathrm{H}), 1.96$ (ddd, $J=14.0,9.0,5.5 \mathrm{~Hz}, 1 \mathrm{H}$, cyclopentane-H), $1.76(\mathrm{~d}, J=1.1 \mathrm{~Hz}, 3 \mathrm{H}$, thymine methyl).

${ }^{13} \mathrm{C}$ NMR $\left(101 \mathrm{MHz}\right.$, DMSO- $\left.d_{6}\right) \delta 176.3,164.2,151.6,140.8,137.9,128.5,128.4,126.9,109.9$, 53.6, 47.2, 45.3, 35.6, 33.9, 12.8.

The carboxylic acid carbon is not resolved. 
IR 3225 (w), 2959 (w), 2860 (w), 1686 (s), 1636 (s), 1449 (m), 1380 (m), 1280 (s), 1213 (m), 1054 (s), 950 (s), 784 (s).

HRMS (ESI) calcd for $\mathrm{C}_{17} \mathrm{H}_{19} \mathrm{~N}_{2} \mathrm{O}_{4}{ }^{+}[\mathrm{M}+\mathrm{H}]^{+} 315.1339$; found 315.1344.

5-Methyl-1-(3-phenyl-4-(((tri isopropylsilyl)oxy)methyl)cyclopentyl)pyrimidine$2,4(1 H, 3 H)$-dione (32).<smiles>Cc1cn(C2CC(C(=O)O)C(c3ccccc3)C2)c(=O)[nH]c1=O</smiles>

59<smiles>Cc1cn(C2CC([PH3+])C(C=[Te])C2)c(=O)[nH]c1=O</smiles>

32

Carboxylic acid 59 ( $0.019 \mathrm{~g}, 0.060 \mathrm{mmol}, 1$ equiv) was solubilized in dry THF $(0.35 \mathrm{~mL})$ in a dried round bottom flask. The reaction mixture was cooled under nitrogen to $0^{\circ} \mathrm{C}$ and a $2 \mathrm{M}$ dimethylsulfide borane solution in THF $(0.076 \mathrm{~mL}, 0.15 \mathrm{mmol}, 2.2$ equiv) was added dropwise. The reaction was stirred at $0{ }^{\circ} \mathrm{C}$ for 5 hours, then it was quenched by addition of a $1 \mathrm{M} \mathrm{HCl}$ solution $(1 \mathrm{~mL})$. The mixture was extracted three times with AcOEt $(3 \mathrm{~mL})$ and the organic layers were dried over anhydrous $\mathrm{MgSO}_{4}$. The crude was directly solubilized into dry and degassed DMF ( $0.7 \mathrm{~mL}$ ), imidazole $(6 \mathrm{mg}, 0.09 \mathrm{mmol}, 1.5$ equiv) and TIPSCI (14 mg, 0.072 mmol, 1.2 equiv) were added. The mixture was stirred at room temperature for 6 hours. The solvent was removed under reduced pressure and the mixture was partitioned between water $(2 \mathrm{~mL})$ and AcOEt $(2 \mathrm{~mL})$. The aqueous layer was extracted 3 times with AcOEt and the organic layers were dried over anhydrous $\mathrm{Na}_{2} \mathrm{CO}_{3}$ and concentrated under reduced pressure. The crude yellow oil was purified by column chromatography, starting with pure DCM and then changing gradually to a mixture of $\mathrm{DCM} / \mathrm{MeOH}(9: 1)$, affording the pure protected alcohol 32 (15 mg, $0.033 \mathrm{mmol}, 55 \%$ ) as a colorless oil.

$\mathbf{R F}(\mathrm{DCM} / \mathrm{MeOH}(9: 1))=0.58$

${ }^{1} \mathrm{H}$ NMR $(400 \mathrm{MHz}$, Chloroform- $d) \delta 8.54(\mathrm{~s}, 1 \mathrm{H},-\mathrm{NH}), 7.32-7.09(\mathrm{~m}, 6 \mathrm{H}, \mathrm{Ar}-\mathrm{H}$ and thymine vinyl-CH), 5.05 (dtd, $J=11.6,9.4,6.5 \mathrm{~Hz}, 1 \mathrm{H}$, cyclopentane- $\mathrm{NCH}), 3.42-3.21\left(\mathrm{~m}, 3 \mathrm{H}, \mathrm{CH}_{2} \mathrm{O}\right.$ and cyclopentane- $\mathrm{CH}$ ), $2.46(\mathrm{td}, J=9.0,4.7 \mathrm{~Hz}, 1 \mathrm{H}$, cyclopentane- $\mathrm{CH}), 2.39-2.27(\mathrm{~m}, 1 \mathrm{H}$ cyclopentane- $\mathrm{CH}), 2.27-2.13(\mathrm{~m}, 2 \mathrm{H}$, cyclopentane- $\mathrm{CH}), 1.96-1.91(\mathrm{~m}, 1 \mathrm{H}$, cyclopentane$\mathrm{CH}), 1.89(\mathrm{~d}, J=1.2 \mathrm{~Hz}, 3 \mathrm{H}$, thymine methyl), $0.93(\mathrm{~m}, 3 \mathrm{H}, \mathrm{TIPS}), 0.88(\mathrm{~d}, J=4.9 \mathrm{~Hz}, 18 \mathrm{H}$, TIPS).

${ }^{13} \mathrm{C}$ NMR (101 MHz, Chloroform- $d$ ) $\delta 163.5,151.2,139.9,136.6,128.3,128.0,126.4,111.0$, 64.0, 54.6, 44.8, 42.1, 35.3, 32.1, 18.0, 18.0, 12.4, 11.8 .

IR 3170 (w), 2945 (w), 1687 (s), 1469 (m), 1385 (w), 1272 (w), 1126 (w), $884(w)$.

HRMS (ESI) calcd for $\mathrm{C}_{26} \mathrm{H}_{41} \mathrm{~N}_{2} \mathrm{O}_{3} \mathrm{Si}^{+}[\mathrm{M}+\mathrm{H}]^{+} 457.2881$; found 457.2881.

\section{Spectra of new compounds}



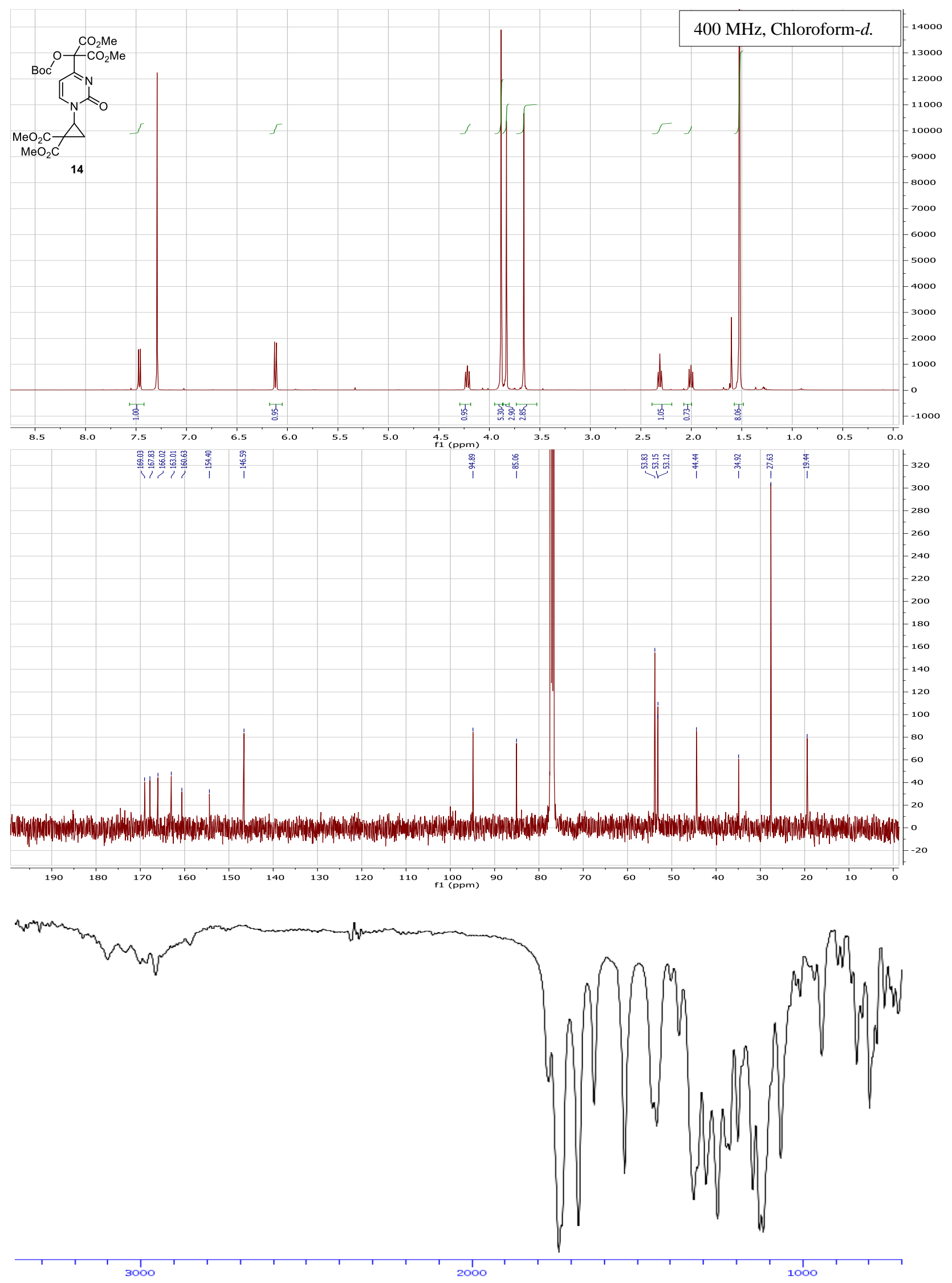

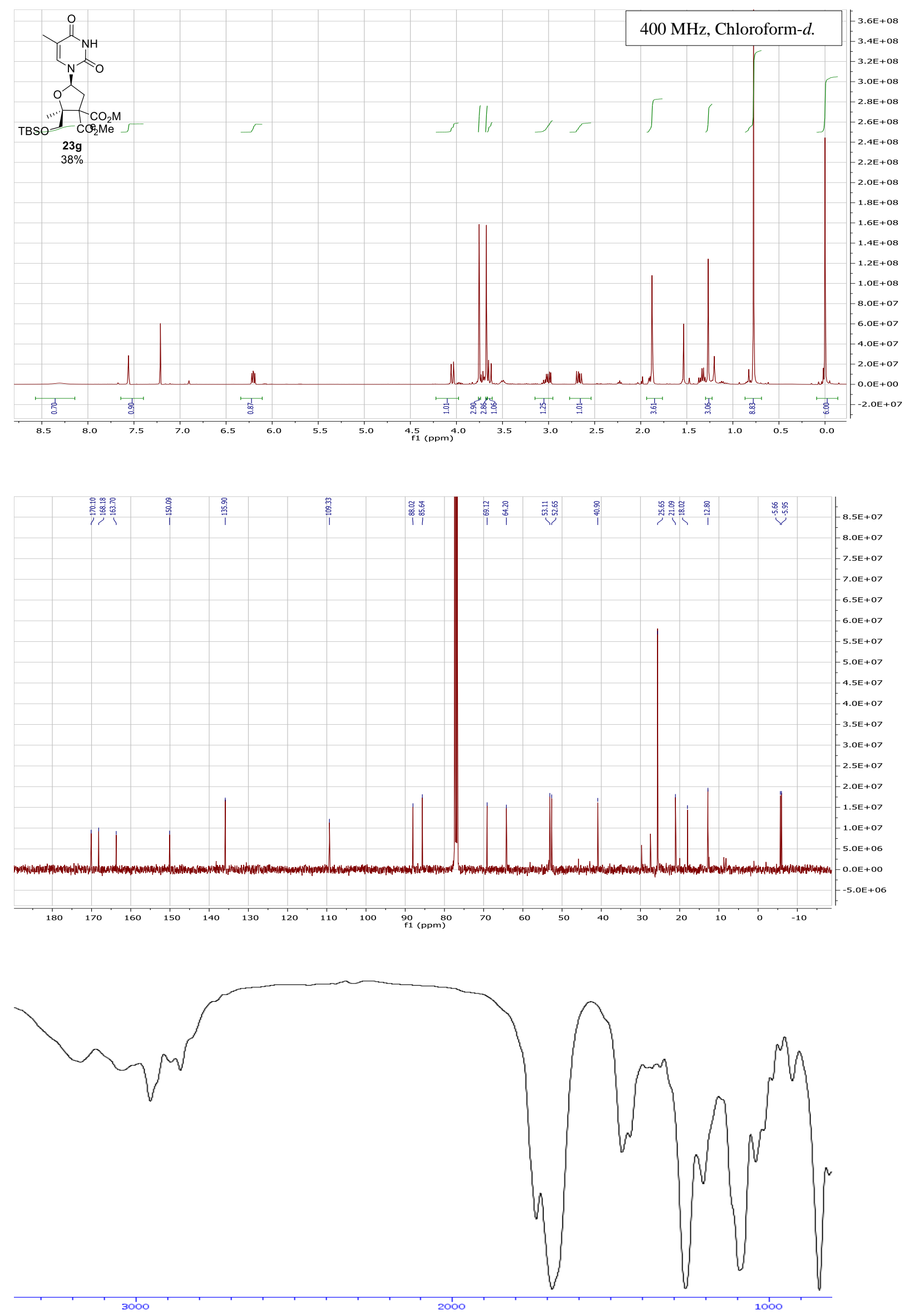

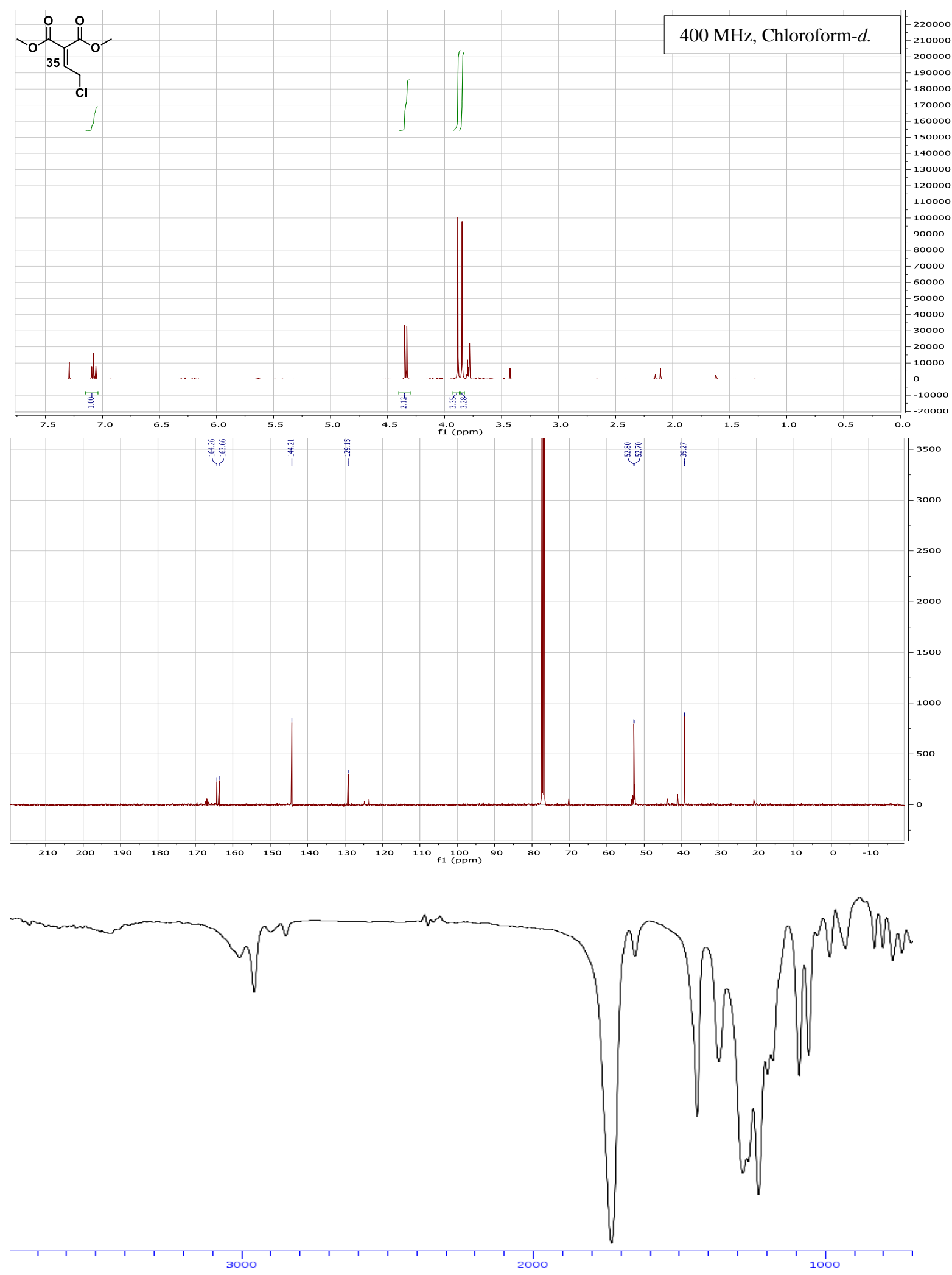

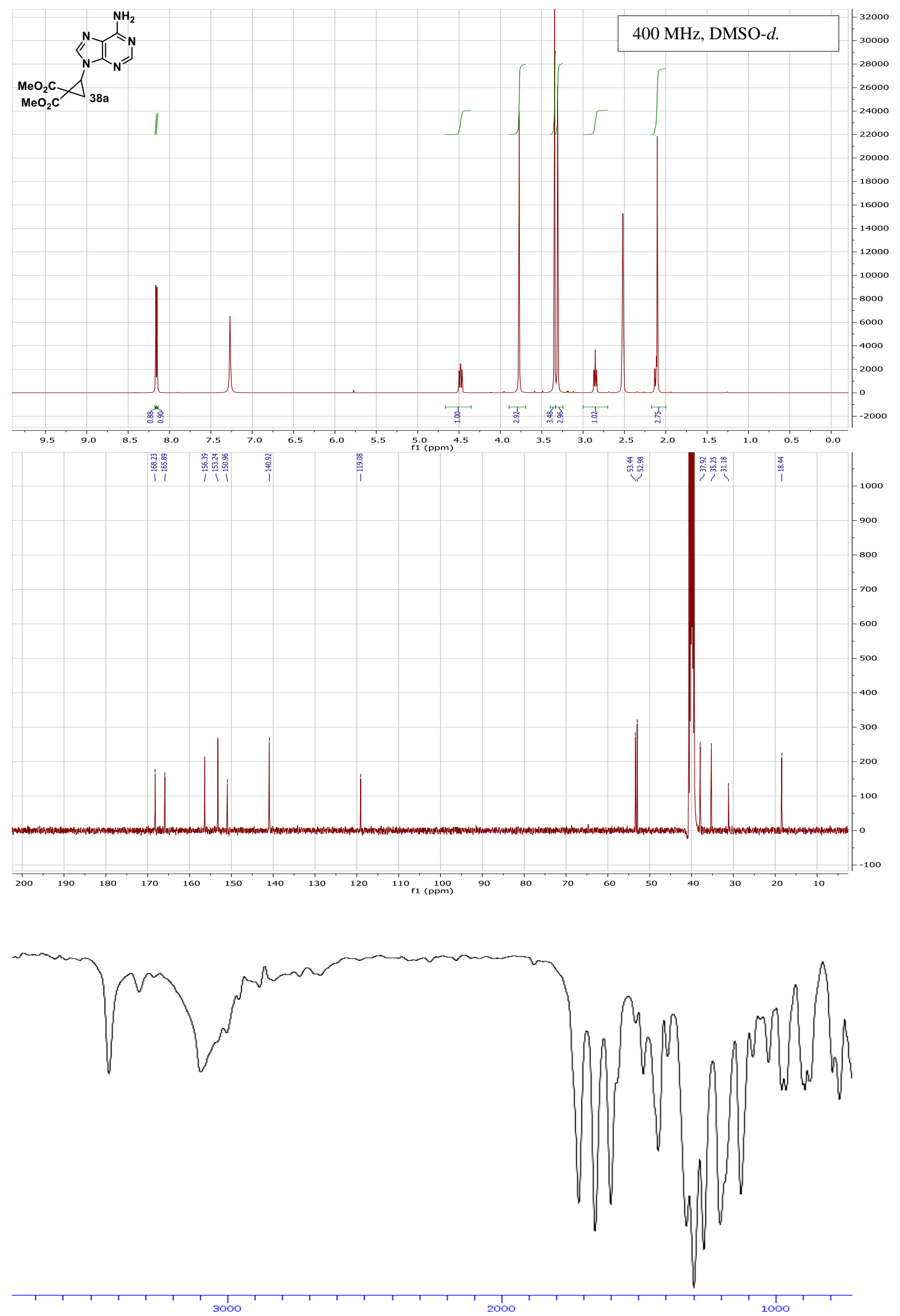

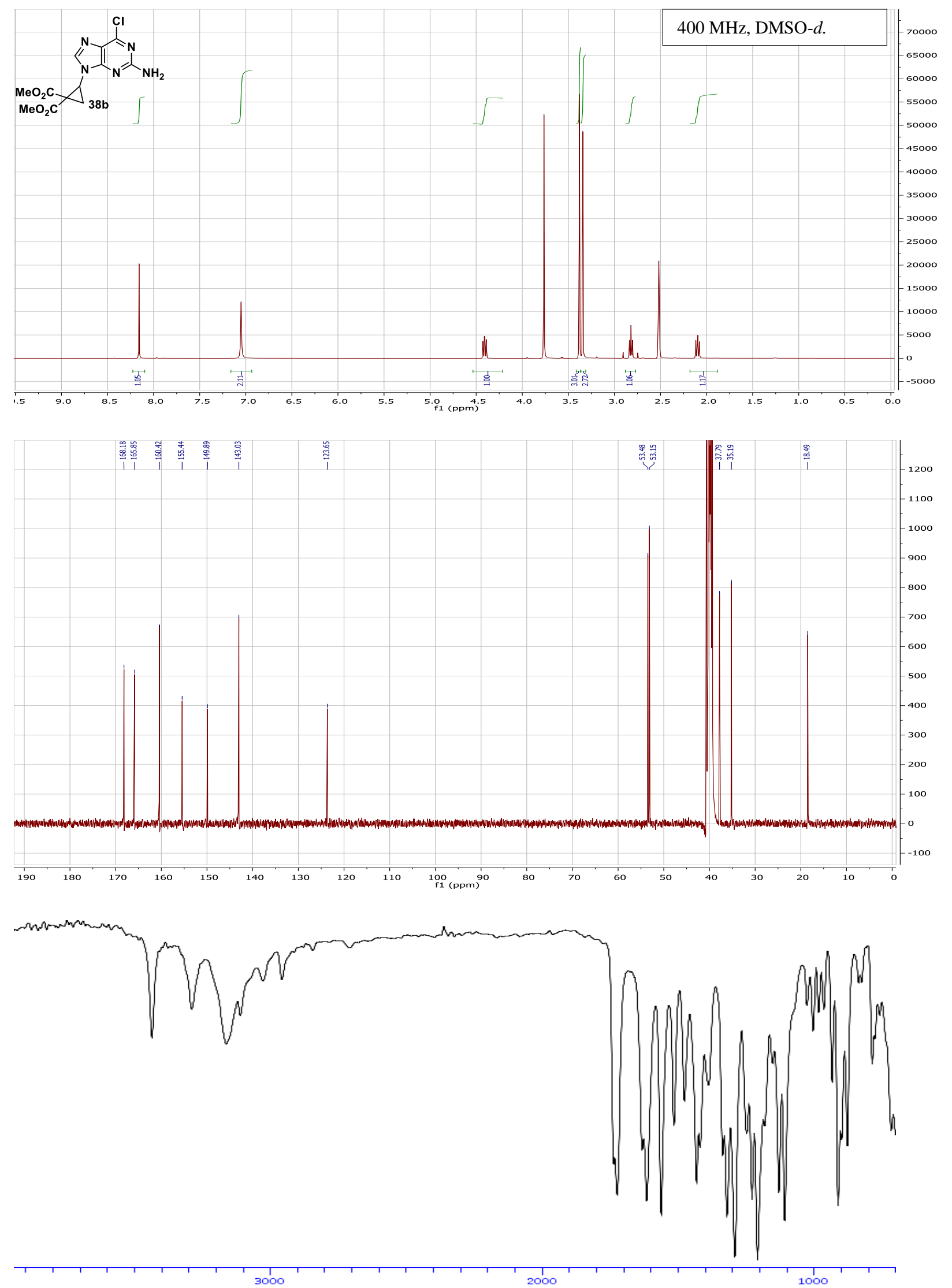

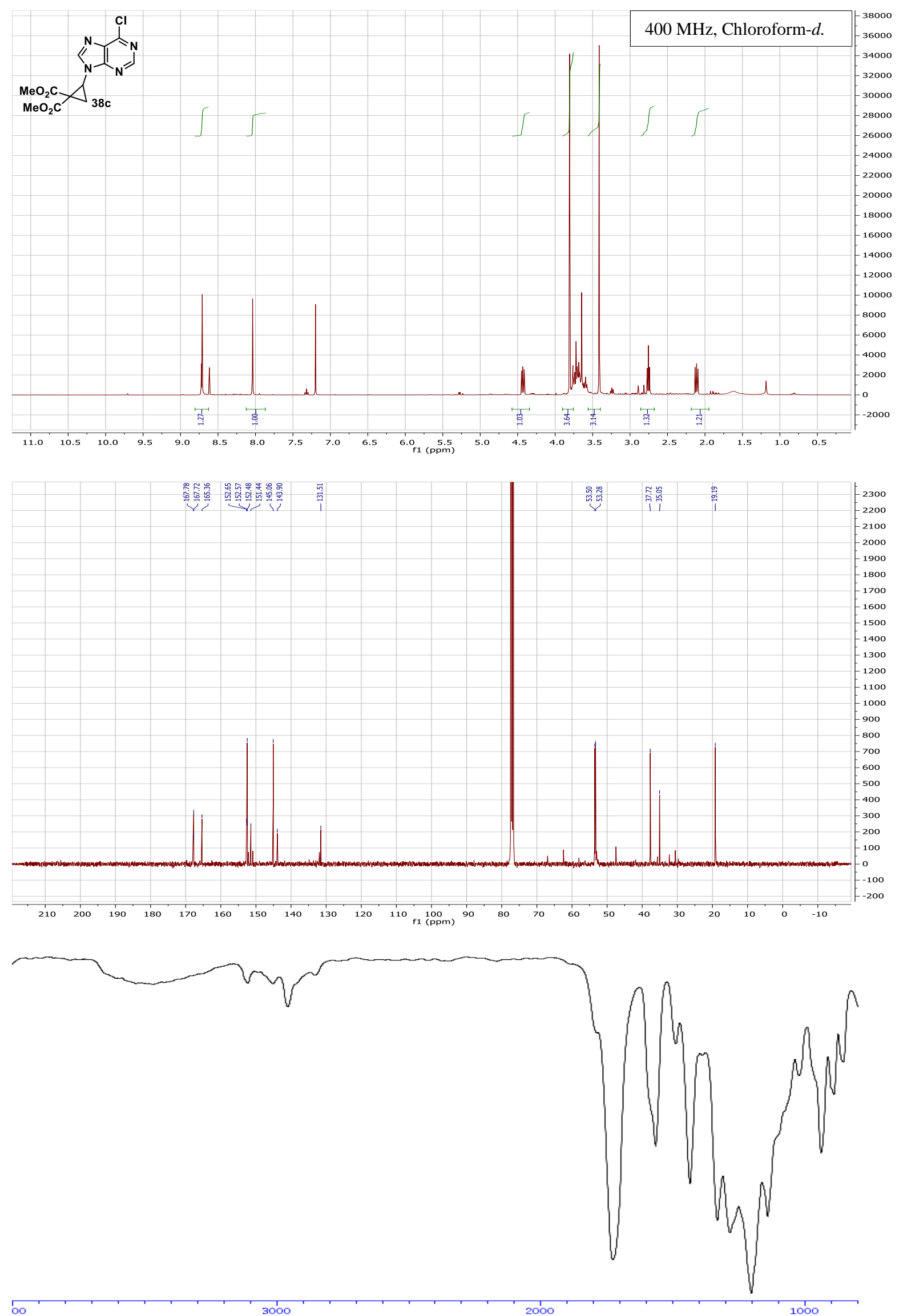

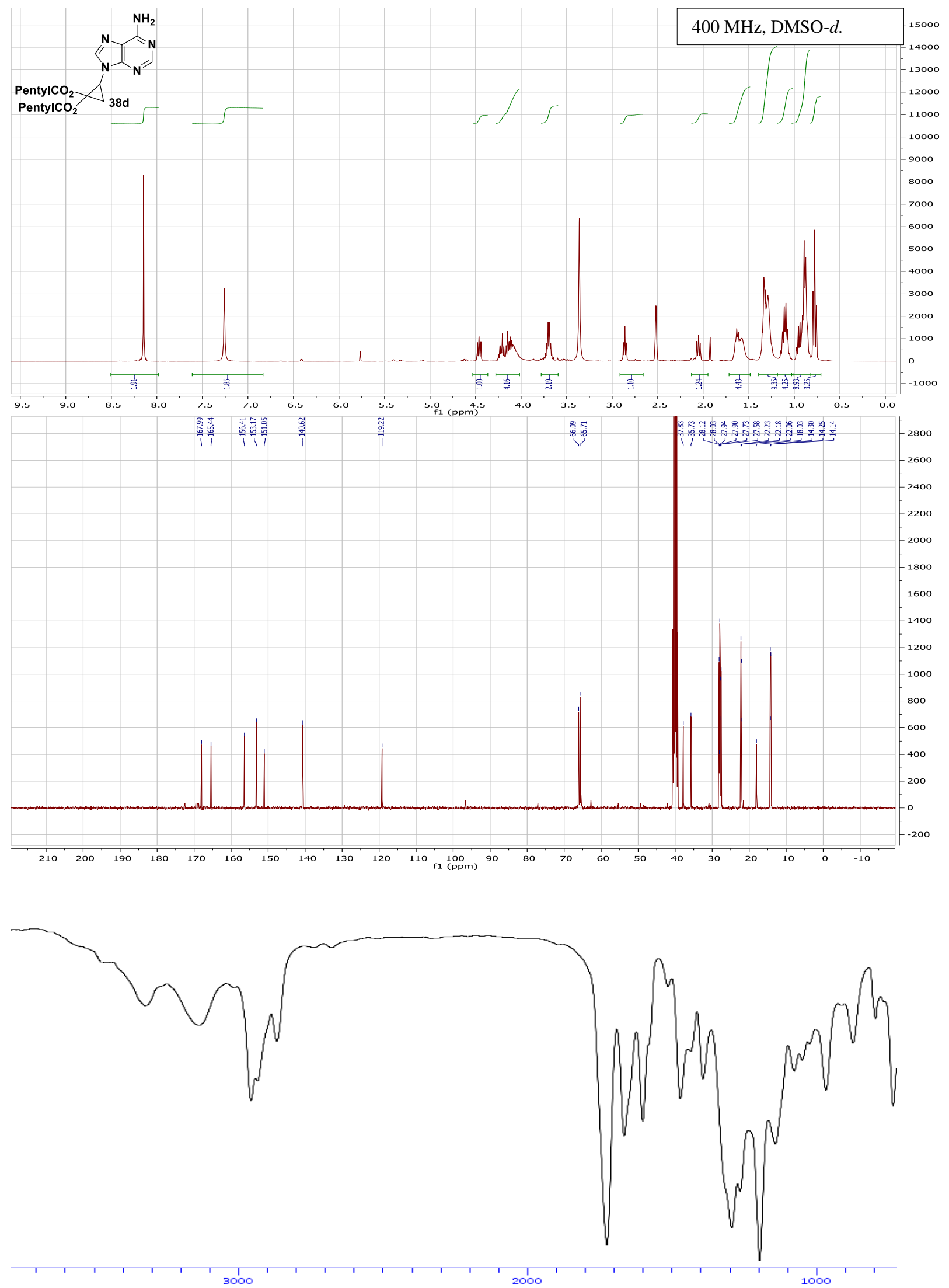

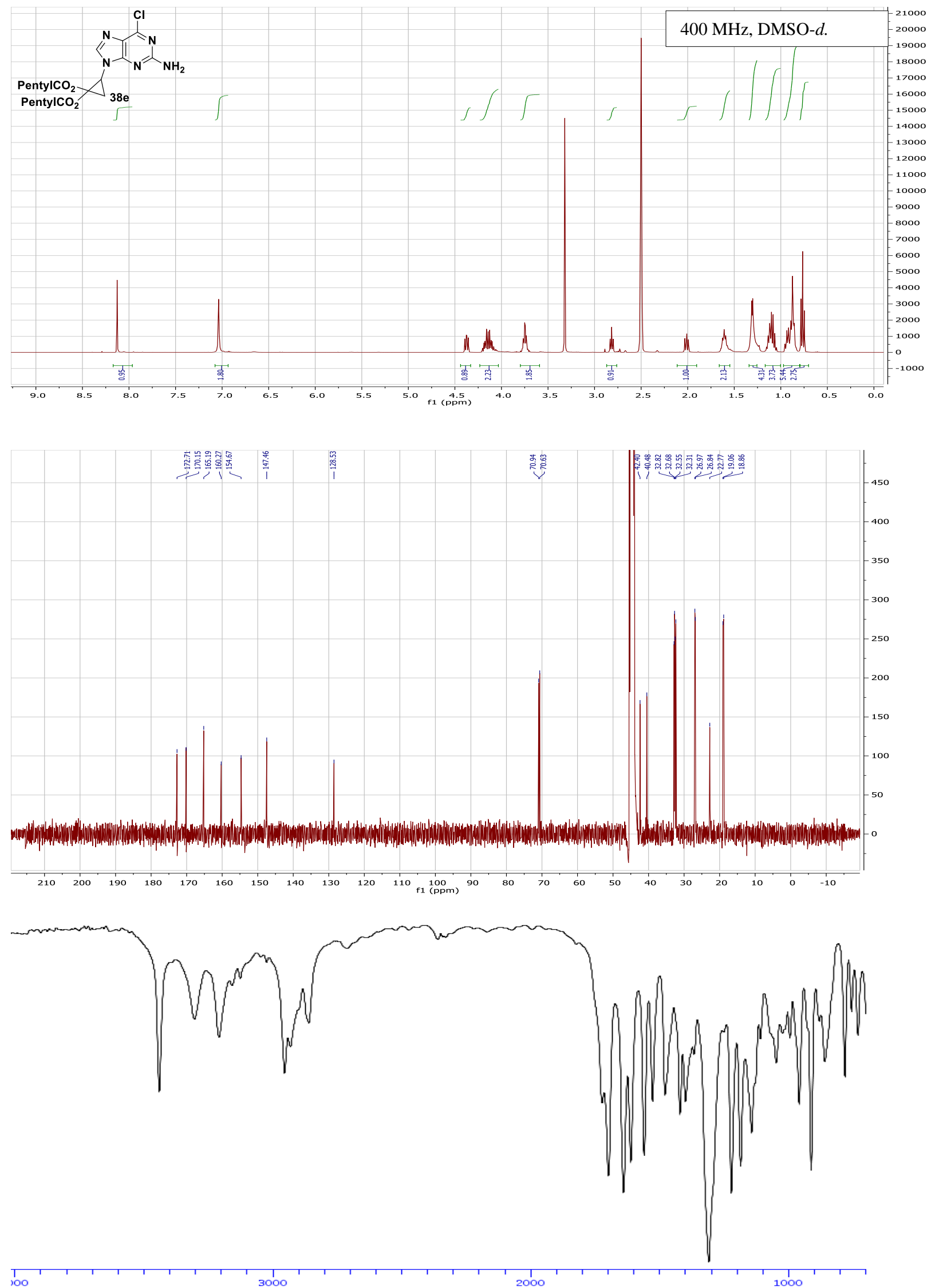


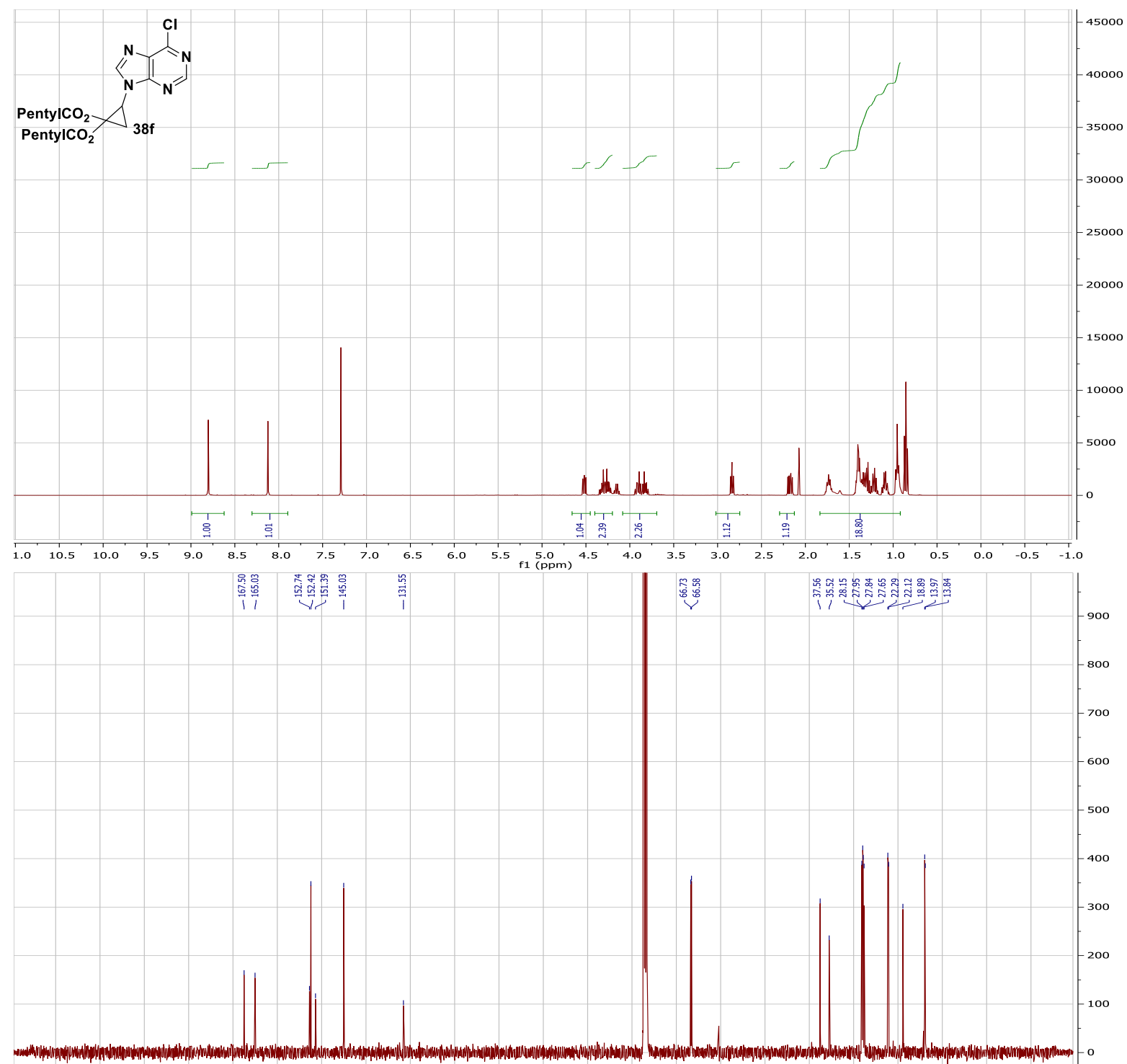

\section{-

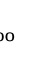




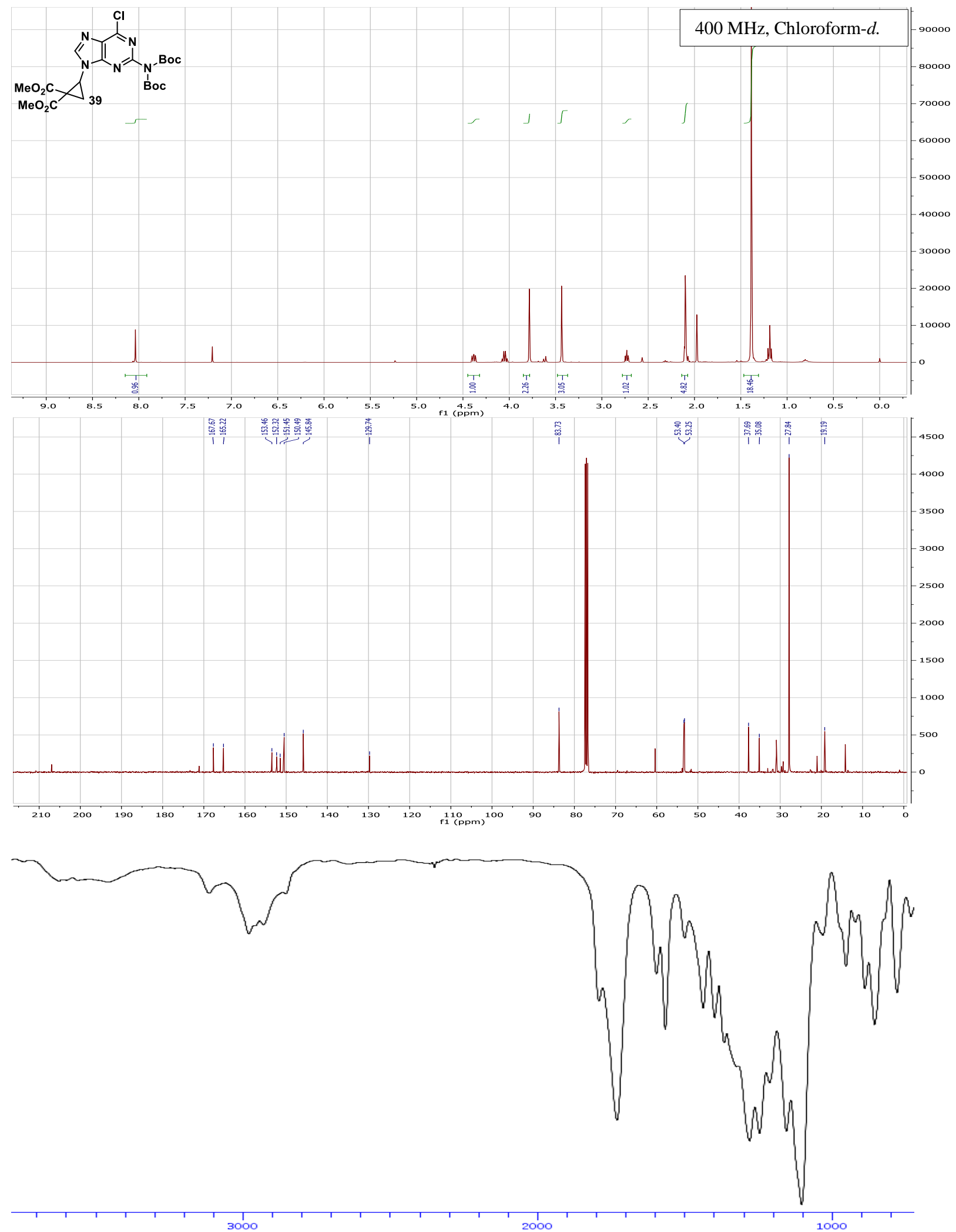

